\title{
Message in a belly Plastic pathways in Fulmars
}

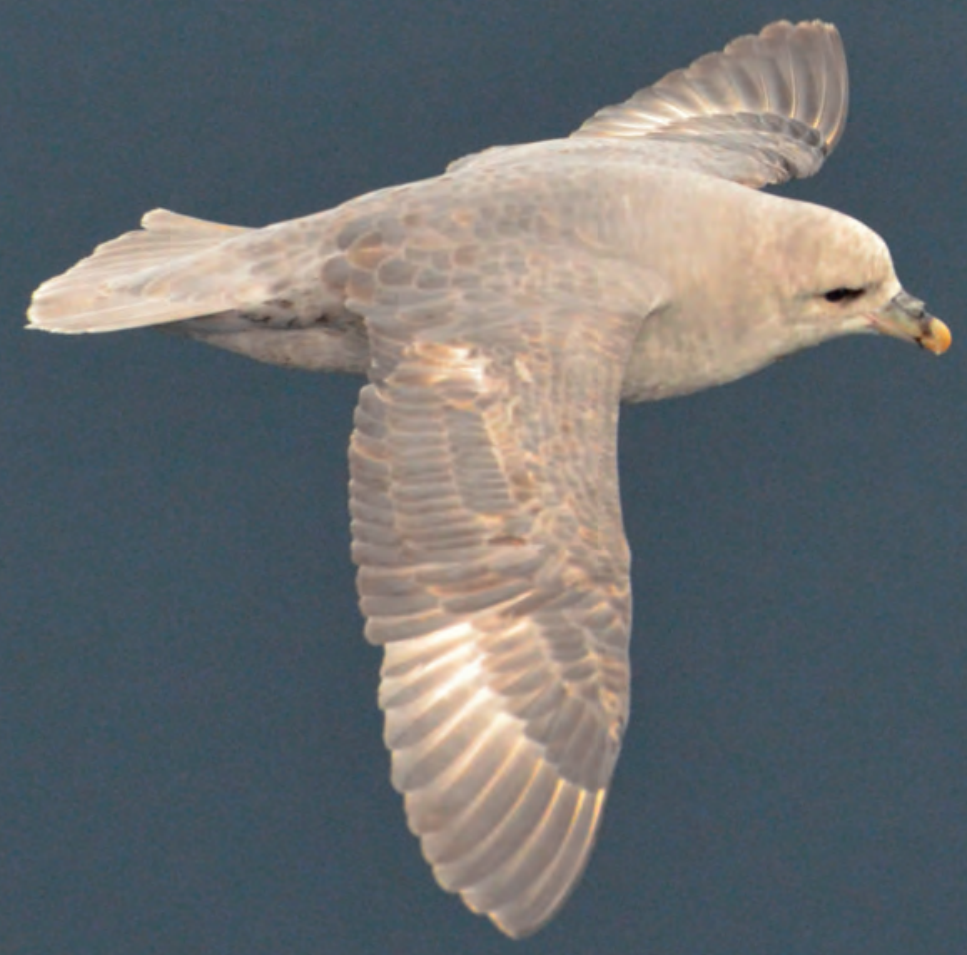

Susanne Kühn 


\section{Propositions}

1. If swallowing a single piece of plastic indeed represented a risk of death of $20 \%$ (Roman et al. 2019), northern fulmars would be extinct by now. (doi: 10.1038/s41598-018-36585-9; this thesis)

2. Fibres impair science.

(this thesis)

3. When relieving tension pneumothorax, the Bülau position should be preferred (Luxem et al. 2013), not only for cosmetic reasons. (ISBN: 978-3437480423)

4. For the people of Tuktoyaktuk, the construction of windmills (CBC News 2009) is like a modern version of Don Quichot's fight.

www.cbc.ca/news/technology/tuktoyaktuk-on-front-line-of-climate-change-1.845397

5. Sharing deliberate falsehood reinforces social coherence of right-winged movements.

6. Editing propositions by anonymous reviewers bears the same risks as brain surgery: creativity and personality vanish.

7. Die Erosion der eigenen Muttersprache kann worden verstärkt durch ähnliche Sprachstrukturen (Schmid 2011). (doi: $10.1017 /$ СBO9780511852046)

8. During the last years, vegetarians switched seats in confession boxes.

Propositions belonging to the thesis, entitled:

Message in a belly - Plastic pathways in Fulmars

Susanne Kühn

Wageningen, 30 October 2020 



\section{Message in a belly Plastic pathways in Fulmars}

Susanne Kühn 


\section{Thesis committee}

\section{Promotors}

Prof. Dr Han Lindeboom

Special Professor Marine Ecology

Wageningen University \& Research

Prof. Dr Geir Wing Gabrielsen

Norwegian Polar Institute, Norway

\section{Co-Promotor}

Dr Jan Andries van Franeker

Marine biologist, Wageningen Marine Research

Wageningen University \& Research

\section{Other members}

Prof. Dr Tinka Murk, Wageningen University \& Research

Prof. Dr Paula Sobral, University of Lisbon (FCT-NOVA), Portugal

Dr Juliana Ivar do Sul, Leibniz-Institute for Baltic Sea Research Rostock, Germany

Dr Eric Stienen, Research Institute Nature and Forest, Belgium

This research was conducted at Wageningen Marine Research under the auspices of the Netherlands Research School of the Socio-Economic and Natural Sciences of the Environment (SENSE). 


\title{
Message in a belly Plastic pathways in Fulmars
}

\author{
Susanne Kühn
}

Thesis

submitted in fulfilment of the requirement for the degree of doctor at Wageningen University

by the authority of the Rector Magnificus,

Prof. Dr A.J.P. Mol,

in the presence of the

thesis committee appointed by the Academic Board

to be defended in public

on Friday 30 October 2020

at 11:00 a.m. in the Aula. 
Susanne Kühn

Message in a belly - Plastic pathways in Fulmars 232 pages

$\mathrm{PhD}$ thesis, Wageningen University, Wageningen, The Netherlands (2020) With references, with summary in English, German and Dutch

ISBN: 978-94-6395-250-7

DOI: https://doi.org/10.18174/509638 


\section{Contents}

Chapter 1 Introduction

Chapter 2 Plastic ingestion by the northern fulmar (Fulmarus glacialis)

Published in Marine Pollution Bulletin 64: 1252-1254 (2012)

Chapter 3 Elevated levels of ingested plastic in a high Arctic seabird, the northern fulmar

(Fulmarus glacialis)

Published in Polar Biology 38: 975-981 (2015)

Chapter 4 Plastic ingestion by juvenile polar cod (Boreogadus saida) in the Arctic Ocean

Published in Polar Biology 41: 1269-1278 (2018)

Chapter 5 The use of potassium hydroxide $(\mathrm{KOH})$ solution as a suitable approach to isolate plastics ingested by marine organisms

Published in Marine Pollution Bulletin 115: 86-90 (2017)

Chapter 6 Details of plastic ingestion and fibre contamination in North Sea fishes

Published in Environmental Pollution: Vol 257 (113569) (2020)

Chapter 7 Global and temporal variation of polymer types ingested by the northern fulmar and southern hemisphere relativess

Accepted in Environmental Science and Pollution Research (2020)

Chapter 8 Marine microplastic: Preparation of relevant test materials for laboratory assessment of ecosystem impacts

Published in Chemosphere 213: 103-113 (2018)

Chapter 9 Transfer of additive chemicals from marine plastic debris to the stomach oil of northern fulmars

Accepted in Frontiers in Environmental Science (2020)

Chapter 10 Quantitative overview of marine debris ingested by marine megafauna

Published in Marine Pollution Bulletin 151: Vol 151 (110858) (2020)

Chapter 11 Synthesis

References

Addenda

Summary/ Samenvatting / Zusammenfassung

Acknowledgements

Author affiliations

List of publications 


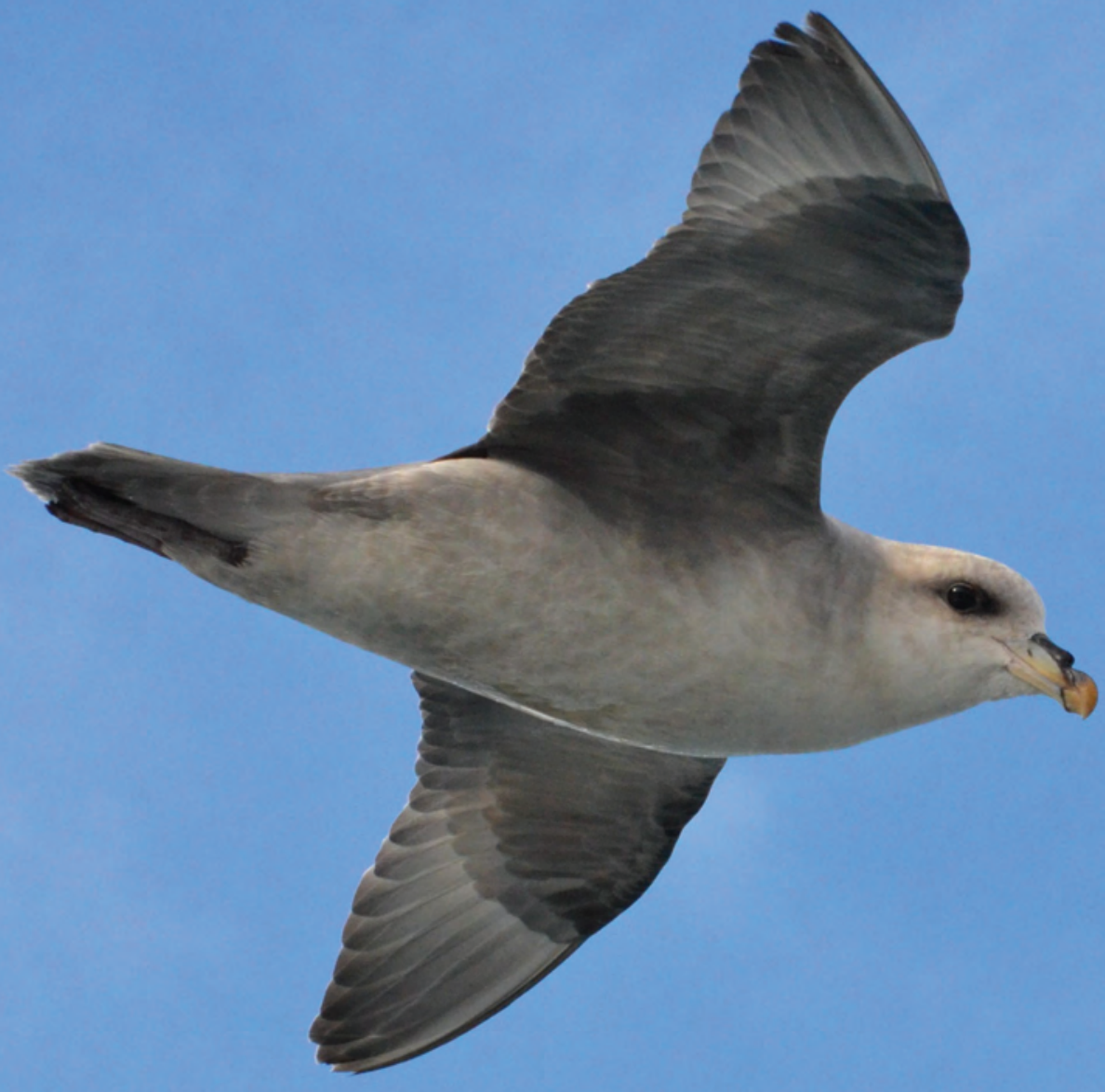


Chapter 1

Introduction 
This $\mathrm{PhD}$ study was conducted as a Dutch component in the PLASTOX project within the European Union Joint Program Initiative on Oceans (EU JPI Oceans). In PLASTOX, 15 scientific institutes from 11 countries collaborated, in order to investigate the direct and indirect ecotoxicological impacts of microplastics on marine organisms. The focus in the PLASTOX project was food web transfer of plastics and the effect of plastic-associated chemicals on marine organisms. For this purpose, and to enhance the comparability of future studies, PLASTOX aimed for the use of shared standardized pristine and litterderived plastics in exposure studies and risk assessments (Booth 2017). In this thesis, these topics are applied to a marine top predator and well-known consumer of plastic debris: the northern fulmar (Fulmarus glacialis).

\section{The fulmar}

The first written accounts of northern fulmars originate from the Icelandic island of Grimsey in 1640 and the island of St. Kilda in northern Scotland. Their occurrence further north in the Arctic is narrated in old sagas of the Inuit of Greenland. While albatrosses, which occur in southern seas, were believed to represent the souls of deceased sailors, the fulmar embodied the northern equivalent of this believe. Arctic whalers believed the

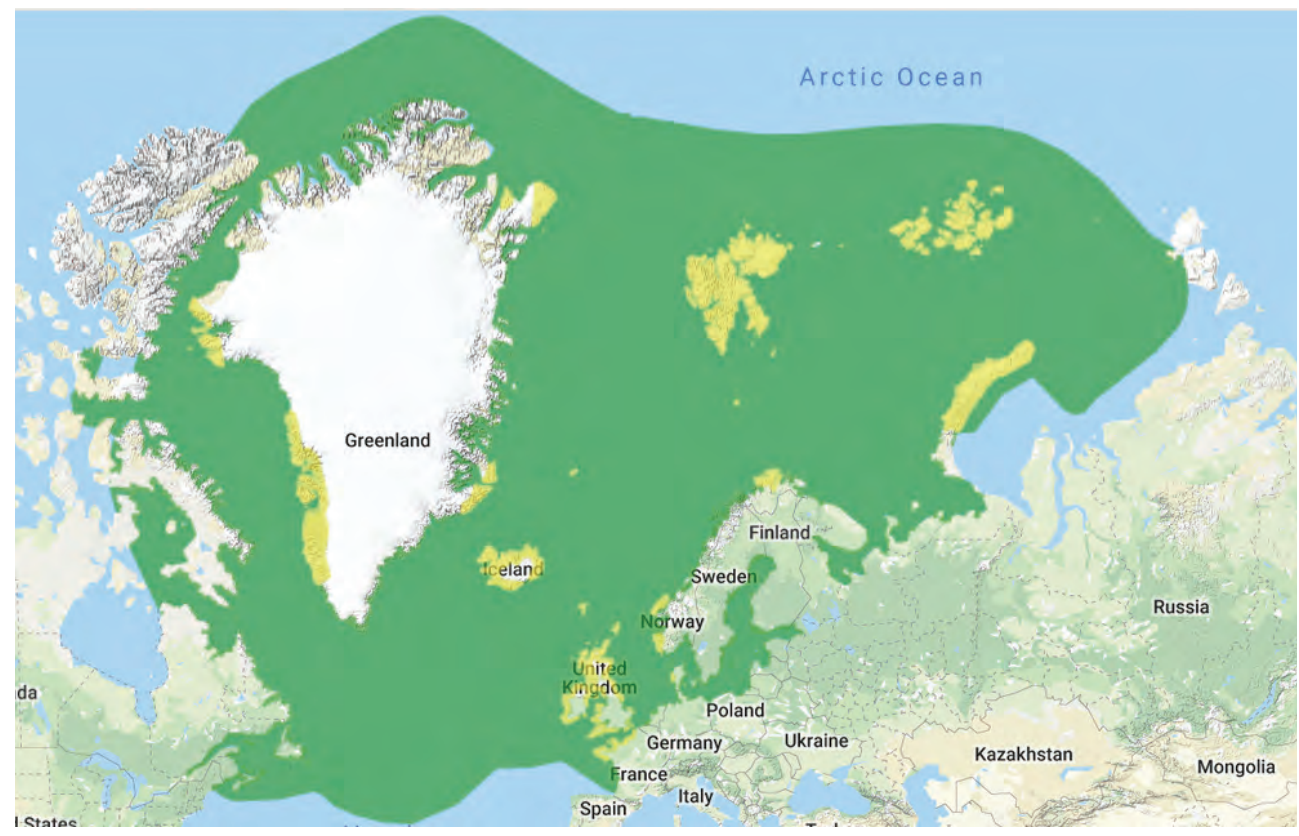

Figure 1.1. North Atlantic distribution range of northern fulmars (Fulmarus glacialis). Green represents the current pelagic distribution and yellow areas indicate known breeding areas (Source: BirdLife International 2019). 


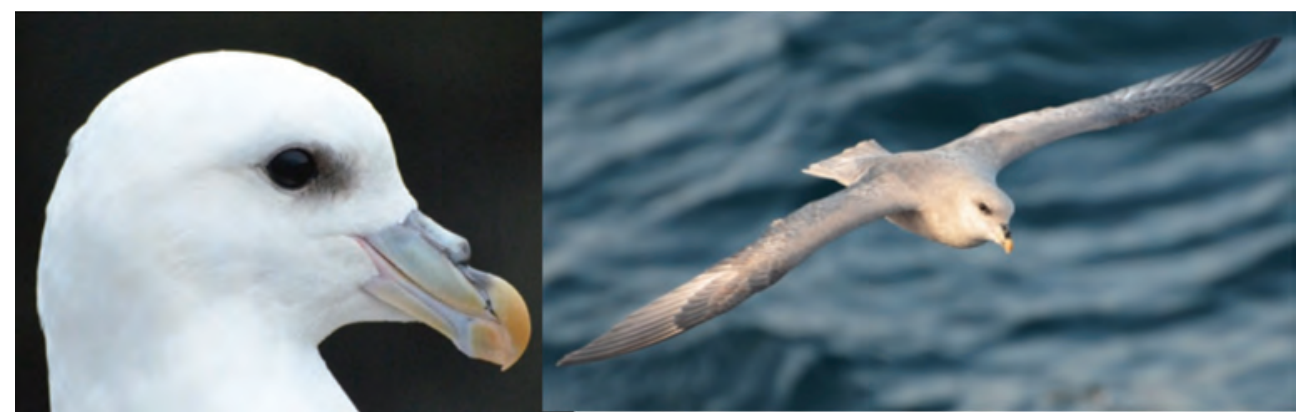

Figure 1.2. Left: Fulmar in portrait with conspicuous tube on the bill. Right: Fulmar in flight. Note chubby body and grey appearance, indicating an Arctic origin.

fulmars to be Greenlandic seamen 'freed from the burden of their ships', referring to their effortless-looking flight (Nicolson 2018). Nowadays, fulmars occur across the northern North Atlantic Ocean, from temperate climate zones (subspecies F. g. auduboni) to the high Arctic (F. g. glacialis) (Figure 1.1). A third subspecies of the fulmar (F. g. rodgersii) can be found in the Northern Pacific boreal and arctic regions. Apart from Grimsey and St. Kilda, the wide distribution of fulmars in the Atlantic temperate climate zone is a relatively recent phenomenon, which occurred in only a few centuries (Fisher 1952). The development of industrial whaling and fishing fleets in combination with the opportunistic and flexible foraging behaviour of fulmars are thought to be behind this range expansion (Fisher 1952).

\section{Fulmar characteristics}

Fulmars have a compact body and, relative to their body size, large and stiff wings (Figure 1.2). There is a considerable variation in plumage colour within fulmar populations. Birds from the subspecies auduboni in Europe, Iceland and up to west Greenland and Jan Mayen are white on their head, neck, underwings and belly. Only the upper wings and part of the upper tail show a grey tone. An overall grey plumage becomes increasingly prevalent in latitudes further north. Variable proportions of fulmars of the subspecies glacialis from northern Greenland, Canada, Bear Island Svalbard and the Russian Arctic appear darker, even becoming monochrome grey all over their body (Van Franeker \& Wattel 1982).

Fulmars are related to the bird family of the albatrosses, the best known representatives of the order of tubenosed birds (Procellariiformes), recognized by the possession of tubes on their bill, rather than the open nostrils seen in other bird groups. The fulmar has a single pronounced tube on top of its strong and sharply hooked bill (Figure 1.2). Although related to the strong olfactory capability of tubenosed birds (Carboneras 1992), the nostril tubes in procellariform seabirds are of particular use with estimating wind strength and direction and airspeed. Knowledge of airspeed is crucial for procellariform seabirds, because they have to travel and feed in fast and unpredictable wind circumstances close to the waves (Pennycuick 2008). 
With their long and stiff wings, fulmar utilize the upward winds directly above the waves, mirroring the motion of waves underneath. From a wind force above 4 Beaufort, fulmars are able to soar quickly over large distances without spending much energy. With calm winds, fulmars often have to rest on the water. In this way, the bird can sustain itself until strong winds make searching for food efficient again (Furness \& Bryant 1996). In comparison with other birds, fulmars from the Arctic have a lower basal metabolic rate relative to their body weight and are well insulated to cope with cold climate (Gabrielsen et al. 1988; Ellis \& Gabrielsen 2002).

\section{Fulmar diet}

Fulmars are opportunistic and generalist predators. Already in early Arctic whaling in the $16^{\text {th }}$ century the whaling ships were followed by fulmars, as whalers often threw the offal overboard upon which the fulmars would feed (Fisher 1952; Thompson et al. 1995). Such behaviour increased after the development of steamships in the late 19th century, which strongly increased the whaling industry. When whale populations approached extinction the offal and discards from traditional and industrial fisheries provided an alternative potential man-supplied food to fulmars. Nowadays, fulmars depend less on fishing offal, although the degree of dependency may vary by location and fishing intensity (Camphuysen \& Garthe 1997; Phillips et al. 1999). Fulmars are typical surface feeders: they sit on the water and seize prey from the surface (Mallory et al. 2012). In general, the diet of fulmars consists of fish (gadoids, clupeids, ammotidydae etc.), squid, crustaceans and polychaetes, in varying proportions (Mehlum \& Gabrielsen 1993; Garthe et al. 2004; Mallory et al. 2010; Ojowski et al. 2001; Cherel et al. 2001; Phillips et al. 1999).

Fulmar bills have a very sharp pointed hook which they use to catch and hold prey (Schreiber \& Burger 2001) but also to rip pieces from, e.g. large fish, carcasses of other birds or marine mammals, or to only take the most energy-rich parts of prey, e.g. from commercial fishing offal or from jellyfish (Camphuysen et al. 1995; Byrkjedal \& Langhelle 2019). Although not as strong developed as for example in prions (Prince 1980; Klages \& Cooper 1992), fulmars exhibit small, baleen-like structures along the inside of their bill, allowing them to forage on tiny prey or even fish oil droplets on the water surface.

\section{Digestion processes}

Fulmars have two stomachs. The first is the proventriculus, which is a large, bag-like stomach, where food is stored and digestive processes are catalysed by the addition of stomach fluids. From the proventriculus, food is pushed forward to the gizzard through a small constriction. The gizzard is a small, muscular stomach with rough callous lining, where hard prey items, such as fish bones and otoliths or squid and polychaete jaws, are ground to dust or broken particles, small enough to pass on into the intestines and ultimately be excreted (Figure 1.3).

Procellariform seabirds depend on patchy food sources, e.g. schooling fish or discards from fishing vessels. This has not only consequences for their behaviour, but also on their digestive processes. Procellariiformes have been found to have stomachs with unusually 


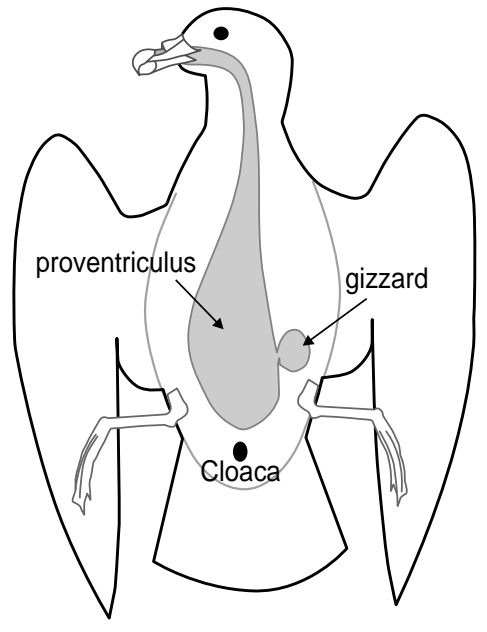

Figure 1.3. Fulmar stomachs (Source: Van Franeker 2004).

low pH values, sometimes as low as 1.5 compared with other birds (Gremillet et al. 2012). Such low $\mathrm{pH}$ values are normally associated with vultures which need an extremely acid stomach environment to digest bones. In albatrosses, however, low $\mathrm{pH}$ is thought to be necessary to digest a large amount of food in a short time, thus creating space for more food intake (Gremillet et al. 2012).

Another unique feature in Procellariiformes is the production of stomach oil. This stomach oil has its origin in fatty prey such as fish or crustaceans. While water is processed quickly, stomach oil (floating on the watery content) is retained in the proventriculus. The oil serves as an energy reserve in addition to body fat, with the advantage that with storing stomach oil no metabolism is required to store the energy (Place et al. 1989). Stomach oil is usually fed by fulmar parents to their chicks, since the semi-digested food is rich in energy and easy to transfer to their offspring. Fulmars are also capable of spitting oil to defend their nests from predators or other invaders (Figure 1.4). This oily defence mechanism can have severe consequences for the attacker, whose feathers may lose water repellence as a result of the oil and thus become waterlogged, resulting in drowning. After experimenting with the efficient defence mechanism in captivity, where several fatalities occurred in other seabirds coming close to spitting fulmars, Swennen (1974) concluded: 'Still it is remarkable that the Fulmar should have such a dangerous weapon at its disposal. The final destruction of an opponent after only a slight confrontation seems rather excessive.'

\section{Two sides of the same coin}

From the above-mentioned aspects, it is evident that fulmars are perfectly designed for a life at (the stormy) sea, true masters of the North Atlantic Ocean. The industrial development of whaling and fisheries may have supported their species expansion. However, recent expansion of industrialized fisheries have coincided with another phenomenon affecting marine wildlife: plastic pollution! 


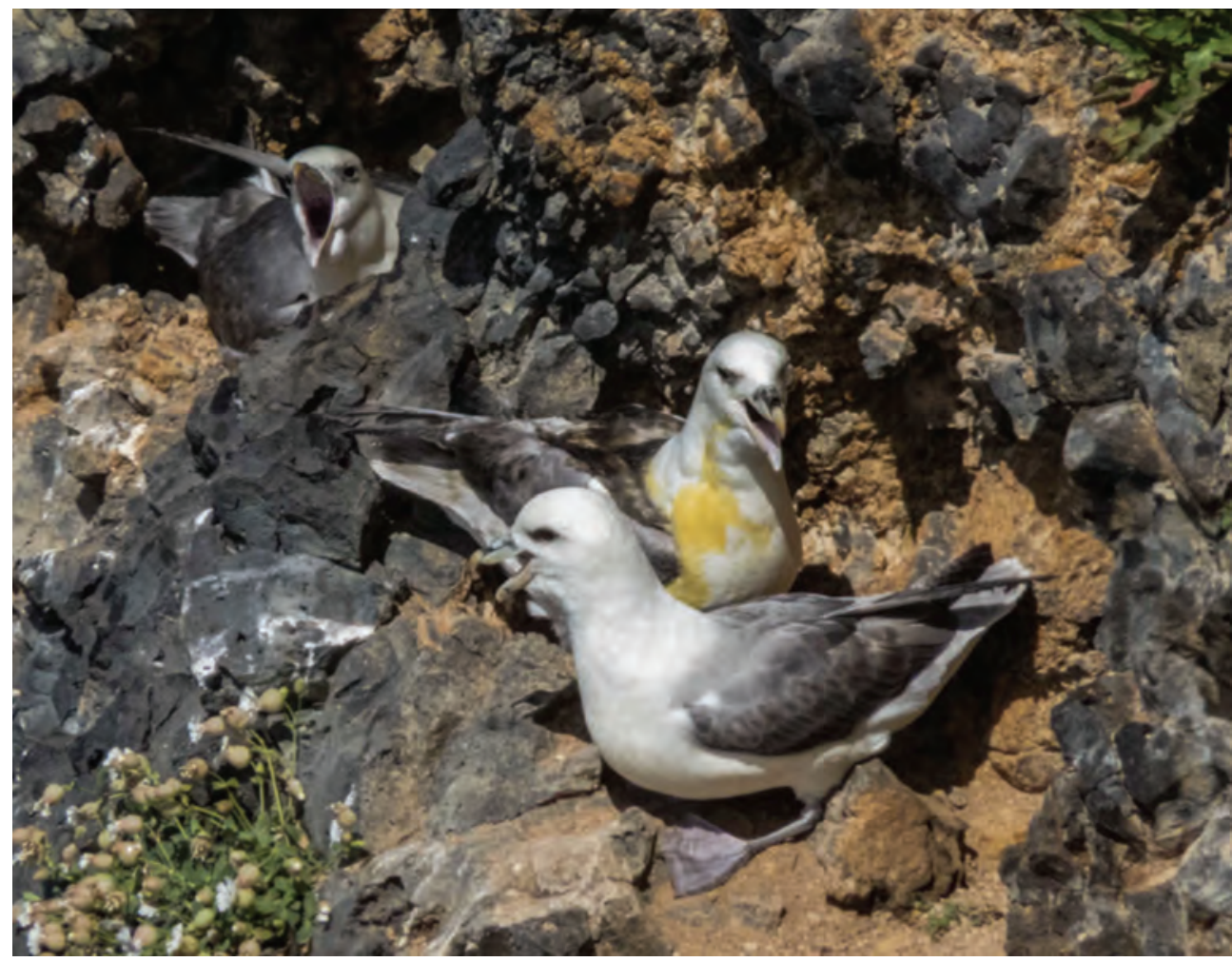

Figure 1.4. Fulmars also spit at each other over nest-site disputes. In this photo it seems the fulmar on the left nest-site has been spitting at its neighbour. Photo by J. van Franeker.

\section{Marine pollution}

\section{Plastic fantastic}

In 1751, European explorers witnessed the use of natural rubber by indigenous people in South America. With a white and sticky juice, derived from a specific tree (Hevea brasiliensis), the indigenous inhabitants were able to build waterproof covers for their bowls and pots (Abts 2010). However the author describes why the first imports of rubber were not very successful: 'this material was not very durable and the smell of the disintegrating rubber made seafarers almost mutiny the ships on the way home to Europe, until the material was thrown overboard' (Abts 2010). It was not until the early $20^{\text {th }}$ century, when the first synthetic rubber was produced, which was mainly used for rubber tyres. In 1907, the first fully synthetic and non-degradable plastic polymer came on the market, called Bakelite. Since then, thanks to their versatile characteristics, polymers have conquered the world. Polyethylene (PE) and polypropylene (PP), the polymers most commonly used nowadays, were synthesized for the first time in 1933 and 1954 (Andrady \& Neal 2009). In 1955, the 
front cover of the lifestyle magazine 'LIFE' showed a young family celebrating single-use plastic, throwing all sorts of items in the air. Using exclusively plastic dishes and cutlery would save allegedly the housewife many hours of a lifetime! And indeed, plastics are often considered extremely beneficial for society (Andrady \& Neal 2009). The authors describe, that the use of plastic bottles instead of glass bottles, supposedly could reduce greenhouse gases and energy consumption in relation to production and transport by $50 \%$. Also, sanitary and hygienic standards improved due to the impermeable properties of plastic (Andrady \& Neal 2009). The industrial production of plastic grew as fast as the number of applications. In 2017, the global plastic production reached 348 million metric tons (PlasticsEurope 2018).

\section{Plastic as stressor}

Although one of the main advantages of plastic is its durability, paradoxically, plastics are continuously being used for single-use items such as plastic bags and food packaging. The stability and non-degradability of the plastic in combination with the often short usage has led to a large amount of waste, much of it ending up or being intentionally dumped into the oceans. Jambeck et al. (2015) estimated that in 2010, between 5 and 12 million metric tons of plastic were discharged into the ocean from sources on land. This waste does not degrade into its original substances but fragments into ever-smaller pieces. Plastic are now showing up on beaches worldwide (Galgani et al. 2015; Lavers \& Bond 2017), in fishing nets (Newman et al. 2015) and, most worryingly, in and around marine organisms (Kühn et al. 2015).

\section{Entanglement}

Entanglement of marine biota in litter has been reported even before plastic was being industrially produced. In 1931 Gudger \& Hoffman (1931) described a shark caught in a rubber car tyre. Gudger collected early accounts of entanglement. For example, in 1938 he reports a cod (Gadus morhua), trapped with its head in an iron can (Gudger 1938). Scordino (1985) studied entangled seals from 1967 onwards. In his 18-year study on St. Pauls Island in Alaska, he counted more than 2000 of harvested subadult male northern fur seals (Callorhinus ursinus) entangled in either fishing gear or packaging straps, annually the equivalent of $0.4 \%$ of harvested subadult males. Since then many species, from small crustaceans (Good et al. 2010) to the large fin whale (Waring et al. 2004; Jackson 2013), have been found to suffer from entanglement. Sources and fate of entanglement have been discussed in earlier reviews (Laist 1997; Kühn et al. 2015; Ryan 2018).

\section{Ingestion}

Although not as visible as entanglement, many individuals from many species are affected by the ingestion of plastics. In 2015, 331 marine species were found with ingested plastics, covering several species groups from invertebrates to marine fish, mammals, turtles and birds (Kühn et al. 2015). The reason of plastic uptake in these species often remains unclear. Plastic may be confused with natural prey, and as ingested plastics often do not 
cause immediate fatalities, a learning effect to avoid plastic is unlikely (Gall \& Thompson 2015; Kühn et al. 2015). Over the last five years (2014-2018), 93\% of fulmars found beached on Dutch coasts contained plastic, with an average of 24 plastic pieces per bird, weighing 0.26 gram (Van Franeker \& Kühn 2019; see also Figure 1.6). Until some decades ago, almost everything floating on the sea surface such as pumice, algae or wood was digestible, although not providing energy (Figure 1.5). For example, albatrosses forage on eggs of flying fish that are attached to non-food material. Previously this consisted of seaweed, wood or pumice, but now it often consists of plastics (Pettit et al. 1981). Besides direct ingestion, there are other ways birds may take up plastics. For example, plastic has been found in several common prey species, resulting in an indirect uptake for their predators, so-called secondary ingestion. This process facilitates trophic transfer of plastic between species and may lead to bioaccumulation in higher trophic organisms. Fish with plastics in their stomach were reported as prey by birds and mammals (Hipfner et al. 2017; Nelms et al. 2018).

There is an ongoing debate on the level of harm caused by ingested plastics (Browne et al. 2015; Werner et al. 2016). Direct fatalities have rarely been described in literature (but see e.g. Brandão et al. 2011; Roman et al. 2019a). More concerning are the potential sublethal effects that may be linked to the uptake of plastics. Sub-lethal effects may occur when potentially hazardous chemicals, associated to plastic, are released into an organism (Rochman 2015). Part of these chemicals are added to the plastics during the manufacturing process and include e.g. plasticizers, inks, UV stabilizers and flame retardants. Other chemicals are sorbed from the surrounding seawater, once plastics enter the ocean (Mato et al. 2001; Teuten et al. 2009). As many factors influence survival and fitness of individuals, populations and species, it is currently impossible to quantify the harm caused by ingested plastics (Browne et al. 2015; Werner et al. 2016). Plastic inside the stomach can lead to reduced fitness in organisms due to reduced food uptake or plastic-associated chemicals. A reduced fitness can lead to increased mortality, particularly when external circumstances, e.g. bad weather conditions or breeding activities, demand extra energy

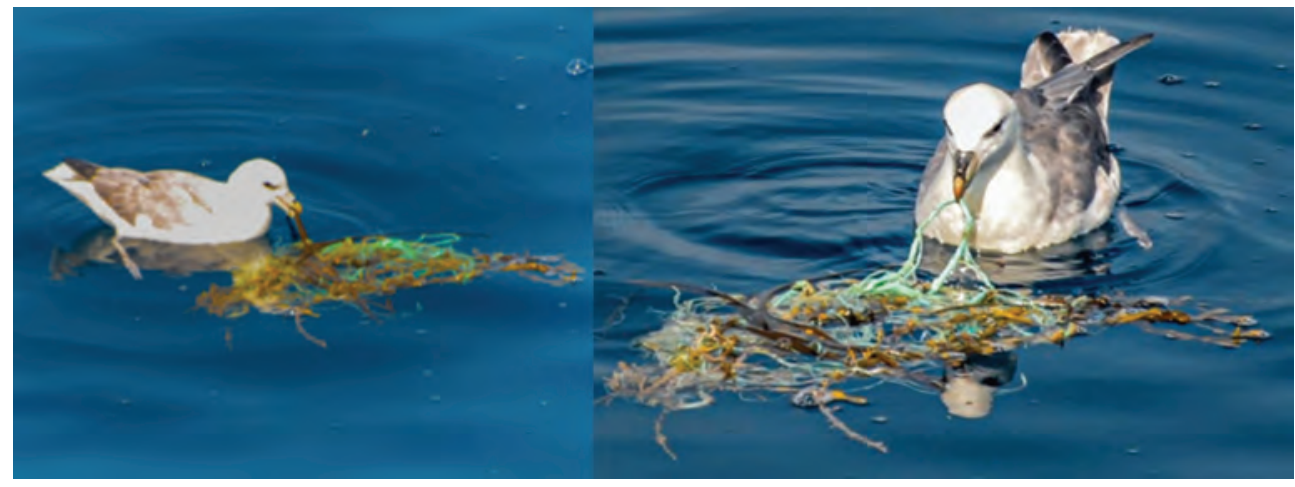

Figure 1.5. Two fulmars investigating the same tangled patch in the North Sea. Left: fulmar nibbling on natural seaweed. Right: fulmar nibbling on a plastic rope. 
(Browne et al. 2015; Rochman et al. 2016). For species in which a large proportion of individuals ingest substantial quantities of plastics, such as the fulmar, the reduced fitness and associated consequences may affect the whole population.

\section{Research on plastic ingestion}

Research on the effects of plastic ingestion is a relatively new field of study. Early studies reported plastics as a curiosity, when encountered within the natural diet of marine wildlife. The oldest dedicated study was published in 1969, when Kenyon \& Kridler (1969) reported the ingestion of plastics in Laysan albatrosses (Phoebastria immutabilis) on Hawaii. These islands in the North Pacific have been a hotspot for plastic accumulation in the surrounding ocean and as a consequence in the stomachs of seabirds breeding there (Moore 2008). In large beached whales, a detailed necropsy is often not possible and therefore only plastic items easily identified visually are reported (e.g. Jacobsen et al. 2010; Abreo et al. 2016). For seabirds, Provencher et al. (2019) recommended to study plastics greater than $1 \mathrm{~mm}$, as smaller particles are thought to be excreted relatively quickly and as the few remaining small items do not contribute much to the total mass of plastics encountered in stomachs. For this reason, stomach analysis using a microscope was deemed to be sufficient to detect ingested plastics for years. More recently, the focus has shifted to the detection of microplastics (Ryan 2015b). These are plastic particles smaller than $5 \mathrm{~mm}$ (Arthur et al. 2009). Fish gained increased attention, as plastic-associated chemicals may be transferred from fish to humans (Rochman et al. 2015). Beside an increased output of scientific studies (Ryan 2015b; Provencher et al. 2017), the increased awareness also led to the development of techniques to separate and identify small plastics. Several techniques have been tested to dissolve organic matter in a sample and retrieve plastics undamaged, including the use of acids, alkalines, dye, and enzymatics (Dehaut et al. 2016). The alkaline potassium hydroxide (KOH) seemed to be the most suitable solution, dissolving organic material but leaving plastics intact (Dehaut et al. 2016; Karami et al. 2017). As the focus of plastic research moved towards smaller particles and their potential hazardous effects, new techniques were required to reliably identify plastics. The most common method is Fourier Transform Infrared Spectroscopy (FTIR; e.g. Amélineau et al. 2016; Avery-Gomm et al. 2016), where the specific polymer type can be established by measuring the reflection of light.

\section{Plastic policy}

The first time plastic pollution played a role at the level of international policy, was in 1972, when the London Dumping Convention came in force. This was followed by the MARPOL (Marine Pollution) Convention, Annex V in 1988, restricting the discard of plastics and other solid waste from ships. MARPOL Annex V has been revised with stricter regulations in 2011 (MEPC 2011). In Europe, additional measures were taken by implementing the EU directive on Port Reception in 2000 (EC 2000). To evaluate whether these 
introduced regulations are successful in reducing the amount of plastics in the oceans, a long-term monitoring program is necessary. In 2002, North Sea ministers decided to include the monitoring of plastic ingestion by seabirds, as one of its Ecological Quality Objectives (EcoQO; North Sea Ministerial Conference 2002. The OSPAR Convention (Oslo-Paris Convention for the Protection of the Marine Environment of the North-East Atlantic) was charged with the EcoQO implementation and decided to use fulmars as the seabird species to be monitored (OSPAR 2008). Dead fulmars commonly strand on beaches around the North Sea and are collected by many volunteers, without whom, this research would be impossible (Van Franeker \& Meijboom 2002; Van Franeker \& Kühn 2019). Fulmars were chosen based on many beneficial characteristics of the species (Van Franeker \& Meijboom 2002; Van Franeker et al. 2011):

- The species is widely distributed over all of the North Atlantic (and North Pacific)

- The species is abundant and is regularly found dead on beaches

- It forages exclusively at sea

- Fulmars regularly ingest debris including plastics

- This species accumulates indigestible items in the stomach, rather than regurgitating them, thus integrating plastic ingestion over a period of time

- In the Netherlands, beached fulmars were analysed for plastic ingestion from the 1980s onwards.

Targets to reduce plastic in fulmars were formulated as one of OSPAR's Ecological Quality Objectives. The target to be achieved has been formulated as: 'There should be less than $10 \%$ of Northern Fulmars (Fulmarus glacialis) having 0.1 gram or more plastic in the stomach in samples of 50-100 beached fulmars from each of 5 different areas of the North Sea over a period of at least 5 years' (OSPAR 2010).

The above is considered as a long-term goal without a fixed target date (OSPAR 2008). In the Netherlands, during the most recent 5-year period from 2014-2018, 43\% of the fulmars exceeded the target value (Van Franeker \& Kühn 2019; Figure 1.6). When this EcoQO was established, the parameters were chosen based on expert judgement: ambitious but not unrealistic.

Currently, the European Marine Strategy Framework Directive (MSFD) demands member states to set goals for a Good Environmental Status (GES) in EU waters. As the fulmar monitoring in OSPAR is in a mature state of implementation, a comparable approach for a long-term goal is likely to be chosen. For regions where fulmars do not occur (e.g. Baltic and Mediterranean Sea), other suitable monitoring species are considered (Bravo Rebolledo et al. 2013; Matiddi et al. 2017; Van Franeker et al. 2018; Bray et al. 2019), however, most protocols for studying marine litter in other species are not considered ready for implementation yet (Galgani et al. 2013b). 

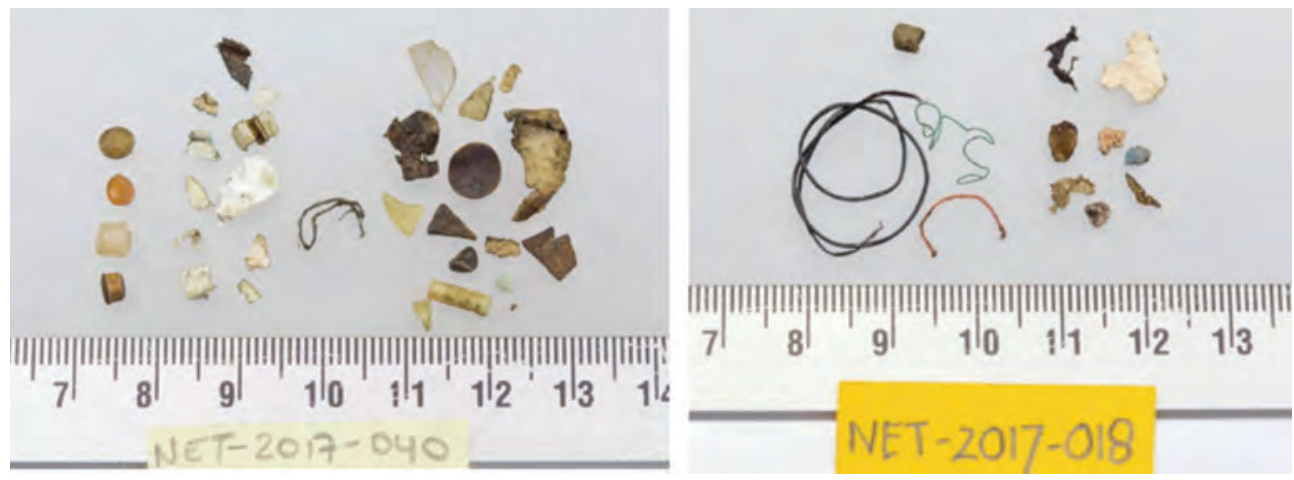

Figure 1.6. Plastics retrieved from fulmar stomachs in the Netherlands. Left, an almost average stomach content is shown with 29 plastic particles, weighing 0.3065 gram. To the right, 12 plastic items, weighing 0.1174 gram were encountered in another fulmar stomach, almost the weight of plastic, OSPAR aims for in their Ecological Quality Objectives. Photos by J. van Franeker.

\section{Outline of this thesis}

The overarching goal of this thesis is to map the different pathways of plastic uptake in fulmars. First, the general uptake of plastics in different fulmar populations is studied. Second, the potential uptake of plastics via prey is examined by studying plastic ingestion of prey fish. The pathway of potentially hazardous, plastic-associated chemicals is studied by the experimental exposure of the fulmars' stomach oil to marine plastic debris.

In order to gain insight into regional variations in plastic ingestion by fulmars, northern fulmars were collected on Iceland and were analysed for plastics in their stomach content. While much was known of plastic in fulmars in the surrounding areas, Iceland still remained uncharted. Results are presented in Chapter 2. Shortly after, a similar research setup was conducted on Svalbard, presented in Chapter 3. For Svalbard, only scarce data from the 1980s was available (Van Franeker 1985; Lydersen et al. 1989).

In addition to directly ingested plastic, prey of the fulmar may have ingested plastic which could indirectly contribute to the plastic load for fulmars. To study the trophic pathways of plastics and gain insight in secondary ingestion, the stomach contents of a key food species of fulmars from the Arctic, the polar cod (Boreogadus saida), was investigated for the presence of microplastic (Chapter 4). Polar cod is an Arctic species which can spend part of his life closely associated to sea ice (David et al. 2015). The Arctic sea ice is an area where plastics are suggested to accumulate (Obbard et al. 2014; Cózar et al. 2017).

While studying microplastic in organisms, researchers have encountered small fibres in their samples. One source of these fibres concerns the process of washing textiles. During the wash cycle, hundreds of thousands fibres are released and sewage systems are not well-equipped to filter out all these fibres before effluent is discharged into the environ- 
ment (Napper \& Thompson 2016). These light-weighed fibres have been also recorded as atmospheric fallouts close to urban centres (Dris et al. 2016), but also in sea ice (Cózar et al. 2017), snow (Bergmann et al. 2019), deep sea sediment (Woodall et al. 2014). The fact that these fibres are so prevalent and widespread in the atmosphere means that there is a possibility for them to settle in samples being investigated for plastics consumption. As these settling fibres cannot be distinguished from fibres ingested by organisms, this secondary contamination implies the potential of overestimation of plastics in samples. Consequently some researchers have excluded microfibres completely from their analyses (e.g. Foekema et al. 2013; Cannon et al. 2016; Forrest \& Hindell 2018). Others decided to include all fibres (Lusher et al. 2013; Vendel et al. 2017), or at least part of them after subtracting the number of fibres found in controls (Lusher et al. 2016; Mizraji et al. 2017; Baalkhuyur et al. 2018). These varying approaches lead to major difficulties when comparing data from different studies. In investigations for plastics in stomachs of fishes in our laboratory, many small fibres were observed, usually suspected to originate from clothing. We studied the proportions of secondary contamination in our samples in Chapter 4 \& 6 .

Recently, the use of potassium hydroxide (KOH) to dissolve organic matter while preserving plastics was proposed (Foekema et al. 2013; Dehaut et al. 2016). Several tests were undertaken, in order to ensure that $\mathrm{KOH}$ is a suitable method to study plastic ingestion. Different plastic and natural test materials were exposed to $\mathrm{KOH}$ (Chapter 5). Using $\mathrm{KOH}$, over 4000 fish from the North Sea were studied for the abundance of plastics (Chapter 6). The species studied were a mix of staple food for marine birds and mammals and commercially marketed species.

Hampering the study of potential negative effects even further, is the heterogeneity of plastics in the environment. Beside some dominant plastic types such as polyethylene and polypropylene, there are countless other types of polymers with different target applications and properties (Andrady \& Neal 2009). During the production process chemicals are added to enhance specific properties of plastics, which include colorants, plasticisers, flame-retardants, UV-blockers, fillers, etc. (Hermabessiere et al. 2017). Once released into the environment, these plastics change their mechanical and chemical properties (Fotopoulou \& Karapanagioti 2012; Gewert et al. 2015). They can become brittle and ultimately break up into even smaller pieces, colours may fade and chemicals integrated into the plastics may leach into the environment, while in contrast, other chemicals from the environment are adsorbed to the plastic (Teuten et al. 2009; Mato et al. 2001). Therefore, each piece of plastic found in the ocean has a unique but mostly untraceable history.

The plastic particles that were ingested by fulmars were studied with infrared spectroscopy for polymer composition. Covering three decades, thousands of particles from fulmars in the North Atlantic were analysed (Chapter 7). Additionally, some plastics ingested by related Antarctic species were included in the analysis. Not only the type of plastics matters, but also its additives, the adsorbed chemicals and the degradation during exposure in the marine environment that characterise every single item of plastic. This complex mixture of properties has led to avoidance of degraded environmental plastics in 
toxicity assessments. Closing the gap between in vitro experiments with pristine plastics and environmental circumstances, plastic macro-debris was collected from the beach in a representative composition. This was milled to micro-sized particles and analysed for future use in experiments (Chapter 8). It is the potentially toxic cocktail that may lead to sublethal effects in fulmars, as plastics are ingested and ground in stomachs in considerable quantities. To test whether degraded plastics leach toxic chemicals into stomach oil of fulmars, the oil was exposed to the created plastic mixture. To assess the time component of leaching effect this was done for varying time periods (Chapter 9).

The frequency of occurrence and the number of plastic pieces ingested in different species may play a relevant role when evaluating the potential harm of marine plastic litter. The body of literature is continuously growing and in order to visualize the extent of the problem, a good overview is crucial. In a review (Chapter 10), an updated list of marine species known to ingest plastics is provided. A major new element is that for seabirds, marine mammals and turtles integrated and species-specific information is provided on frequencies of occurrence and the number of plastic items ingested.

Chapter 11 integrates and discusses all findings from the above-mentioned chapters in the light of implications for science and policy and future research aspects. 


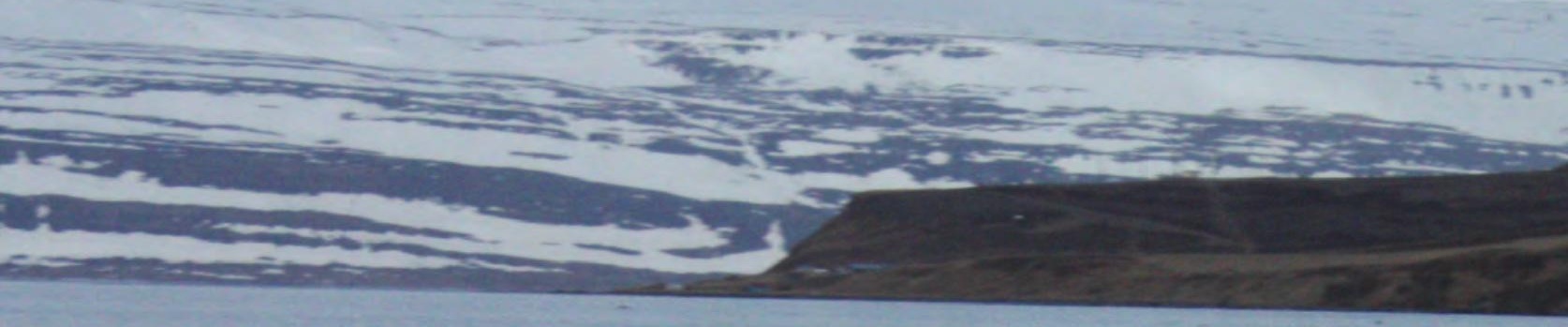

$=$
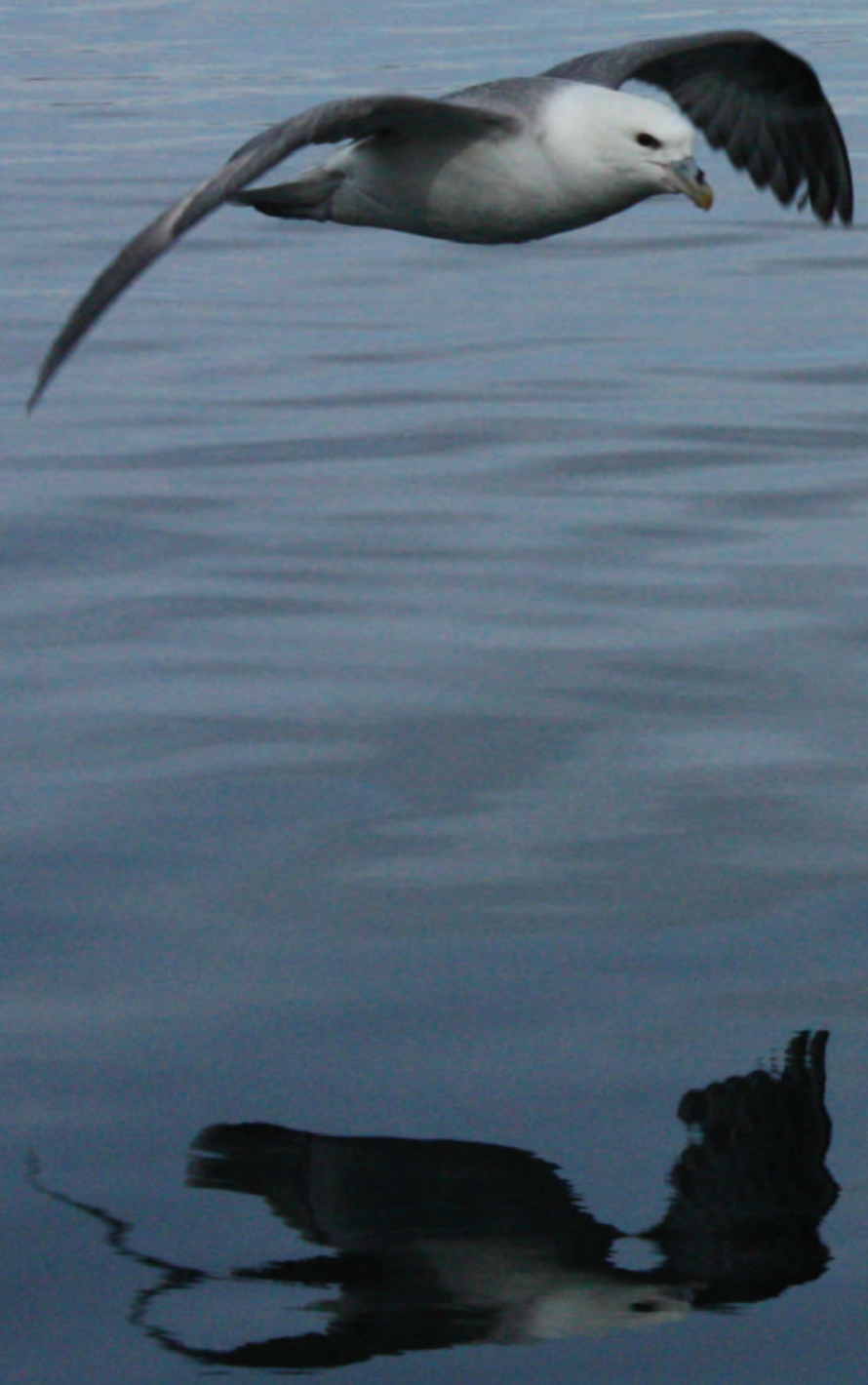


\section{Plastic ingestion by the northern fulmar (Fulmarus glacialis) in Iceland}

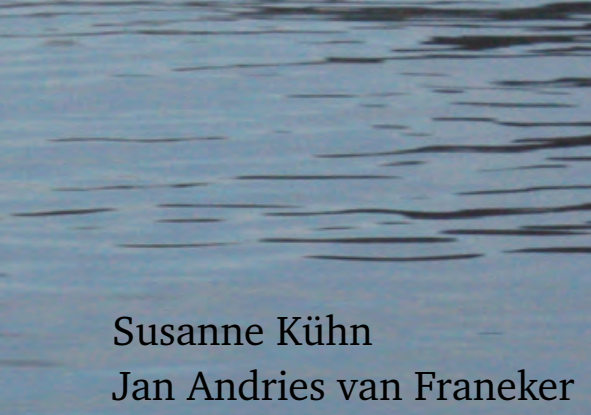




\begin{abstract}
In 2011, northern fulmars (Fulmarus glacialis) from Iceland were used to test the hypothesis that plastic debris decreases at northern latitudes in the Atlantic when moving away from major human centres of coastal and marine activities. Stomach analyses of Icelandic fulmars confirm that plastic pollution levels in the North Atlantic tend to decrease towards higher latitudes. Levels of pollution thus appear to link to regions of intense human coastal and marine activities, suggesting substantial current inputs in those areas.
\end{abstract}




\section{Introduction}

As plastic production increases by $5 \%$ per year and global demand reaches over 265 million tons in 2010 (PlasticsEurope 2011), plastic pollution in the marine environment is likely to remain an controversial topic (Thompson et al. 2009). Besides having economic costs like clean-ups and losses of valuable material, marine plastic debris is known to have many negative environmental impacts (Thompson et al. 2009). Entanglement and ingestion of plastic by various marine species of all trophic levels have been described (e.g. Laist 1987; Derraik 2002). In recent years, a new concern has emerged: the health effects of plastic components and additives on wildlife and humans (Oehlmann et al. 2009). Such concerns emphasize the urgency of establishing monitoring programs for marine plastic litter (Ryan et al. 2009). In the Netherlands, long term monitoring of plastic ingestion by northern fulmars (Fulmarus glacialis) started in the early 1980s and was established as an annual program from the mid-1990s (Van Franeker et al. 2011). The fulmar is a common seabird that is widely distributed around the North Sea and all over the North Atlantic. It forages exclusively at sea, regularly ingests marine debris, generally does not regurgitate solid particles. As a consequence, its stomach contents indicate levels of pollution encountered by the bird over a certain time and space. After 2002, the Dutch research expanded to all other North Sea countries and the Faroe Islands. Monitoring of plastics in fulmars stomachs has become one of the Ecological Quality Objectives (EcoQOs) set by the OsloParis Convention (OSPAR) for the North Sea (OSPAR 2008; Van Franeker et al. 2011; Van Franeker \& The SNS Fulmar Study Group 2011) and is a proposed indicator for Good Environmental Status (GES) in the European Marine Strategy Directive (Galgani et al. 2010). Existing data for the North Atlantic suggested a substantial decrease in marine plastic debris with increasing latitude, but suffered from a major gap in data between temperate waters and high-arctic environments.

\section{Methods}

In 2011, a sample of fulmars from Iceland became available filling that major gap and allowing a test for the hypothesis of a decrease of plastic debris towards higher latitudes in the Atlantic when moving away from major human centres of coastal and marine activities. The 58 fulmars used for this study were accidental bycatch of a long line fishery off Hornstrandir, Westfjords, Iceland in April, 2011. The fulmars were stored at $-20^{\circ} \mathrm{C}$ until dissection in the laboratory of the Natural History Museum of Bolungarvík (Westfjords, Iceland). The dissections were conducted following the Dutch protocol (Van Franeker 2004) including morphometric measurements and data on moult, organ health and body condition. Stomach contents including proventriculus and gizzard were sieved over a 1 $\mathrm{mm}$ mesh with cold fresh water. Plastic particles were taken from the sample and shipped to the Netherlands for further analysis and sorted into categories under a Zeiss stereo microscope. For each plastic category, the number of particles was counted and the weight 
was measured on a Sartorius electronic scale to an accuracy of $0.0001 \mathrm{~g}$. Averages for number and mass of plastics are 'population averages' that include birds without plastic. These averages are mainly presented as arithmetic means and standard error ( \pm se). The relatively small sample and non-normal distributed data with outlying values indicates need for log transformation during analyses. We have done so to calculate geometric mean mass of plastics, adding/subtracting $1 \mathrm{mg}$ of mass at transformation and back-calculation to allow for inclusion of the zero values. Transformation becomes increasingly unsuitable with higher proportions of zero values reducing accuracy in calculations for subcategories of plastic. Another approach to avoid bias from outlying values in non-normal distributions has been chosen in the OSPAR EcoQO by using the percentage of birds having more than $0.1 \mathrm{~g}$ of plastic in the stomach. The EcoQO target for the North Sea is that less than $10 \%$ of fulmars exceed the critical $0.1 \mathrm{~g}$ level (OSPAR 2008; Van Franeker et al. 2011). Regional comparisons were made using 2007-2011 data for the North Sea $(n=682)$, Faroe Islands $(\mathrm{n}=333)$ and Iceland $(\mathrm{n}=58)$ and 2002-2008 data for the Canadian arctic ( $n=169$ ) derived from data in Mallory et al. (2006), Mallory (2008) and Provencher et al. (2009) with additional information from the authors. Statistical tests of regional differences were conducted in GENSTAT $14^{\text {th }}$ edition, using data from individual birds. For Canadian birds individual plastic weight data were only available for a subsample of 25 birds. Differences in plastic weight were evaluated by fitting a negative binominal generalized linear model with and without region included as a factor and differences between those two models were tested using a likelihood ratio test (Venables \& Ripley 2002; Van Franeker et al. 2011). Regional differences in the incidence and EcoQO performance were evaluated similarly using GLM with Bernoulli distribution.

\section{Results and Discussion}

Of the 58 fulmars, 33 birds were male, 25 birds were females. Age characters indicated 49 adult birds versus 9 non-adults of which one bird was juvenile ( $1^{\text {st }}$ year), two birds were $2^{\text {nd }}$ year birds and 6 birds were immature (never bred, 3-c. 6 years old). All birds were of the double light (LL) colour phase (van Franeker, 2004) and all were in good body condition. Data on plastic abundance in the stomachs is given in Table 2.1.

Table 2.1. Abundance of plastic in stomachs of Icelandic fulmars, Westfjords, April 2011.

\begin{tabular}{lrcccccccc} 
& $\begin{array}{c}\text { Sample } \\
\text { size }\end{array}$ & Incidence & $\mathbf{n}$ & $\pm \mathbf{s e}$ & $\mathbf{g}$ & $\pm \mathbf{s e}$ & $\begin{array}{c}\text { Max } \mathbf{g} \\
\text { Geometric } \\
\text { mass }\end{array}$ & $\begin{array}{c}\text { EcoQO } \\
\text { performance }\end{array}$ \\
\hline ALL & 58 & $79 \%$ & 6.0 & \pm 0.99 & 0.13 & \pm 0.04 & 2.0 & 0.020 & $28 \%$ \\
Adults & 49 & $82 \%$ & 5.3 & \pm 0.89 & 0.13 & \pm 0.05 & 2.0 & 0.021 & $27 \%$ \\
Non-adults & 9 & $67 \%$ & 9.7 & \pm 4.13 & 0.14 & \pm 0.09 & 0.8 & 0.018 & $33 \%$
\end{tabular}


Data are also specified for adults and non-adult birds because the long term Dutch study indicates a consistent influence of age on the amount of plastic in the stomach. Details for different categories of plastics are given in Table 2.2.

The Icelandic birds were all victims from long-line fisheries and were in good physical condition, which differs from the beached corpses in North Sea samples. Beached birds have usually died emaciated after prolonged starvation (Van Franeker et al. 2011). However, analyses in the pilot study for the fulmar project (Van Franeker \& Meijboom 2002) showed no statistical difference between plastics in stomachs of emaciated individuals as compared to those that had died instantly in good body condition due to e.g. collisions or drowning. Consequently, data from beached birds from the North Sea can be compared to those from other areas where healthy birds are sampled. The long term fulmar research has clearly shown that younger birds have more plastics in their stomachs than adults (Van Franeker et al. 2011). This could not be confirmed in the current Icelandic sample (Table 2.1), but the sample-size for non-adults is only nine birds. Opposite results for arithmetic averages, the number of items and mass and geometric mean mass indicate that the sample size for age groups are too small. Further data are needed.

The overall data confirm decreasing abundance of plastic in fulmar stomachs with increasing latitude in the North Atlantic. Incidence of plastics in Icelandic stomachs is $80 \%$, further south in North Sea and around the Faroe Islands it ranges between $90 \%$ and $100 \%$ but Canadian Arctic fulmars average at 40\% incidence. Incidence differs significantly between all regions (Faroe-Iceland $\mathrm{P}=0.003$; Faroe-Canada $\mathrm{P}<0.001$ and Iceland-Canada $\mathrm{P}<0.001)$, except between the North Sea and Faroe Islands $(\mathrm{P}=0.085)$. Stepwise decreases from south to north are shown in Figure 2.1. In terms of average arithmetic weight of plastics, the change from Faroese to Icelandic birds (Figure 2.1A) is not so clear due to high outliers in the relatively small Icelandic sample (note large standard error). Plastic weight in stomachs of North Sea fulmars differs significantly from all the other regions $(\mathrm{F}<0.001)$; differences between the others were not significant, due to

Table 2.2. Types of plastic particles in stomachs of Icelandic fulmars, Westfjords, April 2011.

\begin{tabular}{|c|c|c|c|c|c|c|c|}
\hline & Incidence & $\mathbf{n}$ & $\pm \mathrm{se}$ & g & \pm se & $\max g$ & $\begin{array}{c}\text { geometric } \\
\text { mass }\end{array}$ \\
\hline ALL PLASTICS & $79 \%$ & 6.0 & \pm 0.99 & 0.13 & \pm 0.04 & 2.0 & 0.020 \\
\hline Industrial plastic & $19 \%$ & 0.4 & \pm 0.18 & 0.01 & \pm 0.00 & 0.1 & 0.001 \\
\hline User plastic & $78 \%$ & 5.6 & \pm 0.94 & 0.12 & \pm 0.04 & 2.0 & 0.018 \\
\hline sheetlike & $17 \%$ & 0.6 & \pm 0.24 & 0.00 & \pm 0.00 & 0.1 & 0.000 \\
\hline threadlike & $17 \%$ & 0.4 & \pm 0.16 & 0.02 & \pm 0.01 & 0.8 & 0.001 \\
\hline foamed & $29 \%$ & 1.0 & \pm 0.39 & 0.01 & \pm 0.00 & 0.2 & 0.001 \\
\hline fragments & $69 \%$ & 3.4 & \pm 0.59 & 0.07 & \pm 0.02 & 1.3 & 0.011 \\
\hline other & $5 \%$ & 0.2 & \pm 0.16 & 0.03 & \pm 0.03 & 1.6 & 0.000 \\
\hline
\end{tabular}



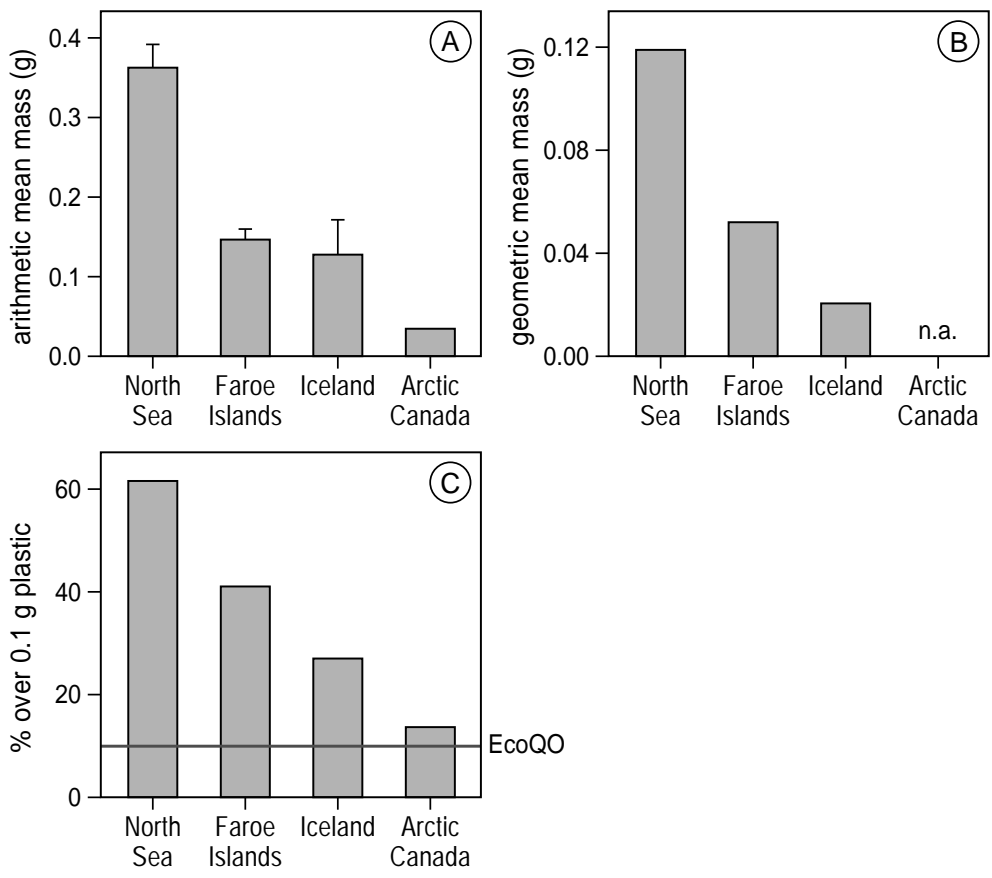

Figure 2.1. Patterns in abundance of plastics by mass in fulmar stomachs in the North Atlantic. $\mathbf{A}=$ arithmetic mean mass \pm standard error; $\mathbf{B}=$ geometric mean mass; $\mathbf{C}=$ proportion of birds having more than $0.1 \mathrm{~g}$ plastic in the stomach (EcoQO performance).

smaller sample sizes in combination with high variability. More pronounced differences are indicated by geometric mean mass of plastics. (Figure 2.1B) because of the reduced impact of high outliers; such effect is also evident when expressing the data as EcoQO percentage of birds having more than $0.1 \mathrm{~g}$ of plastic in their stomachs (Figure 2.1C). EcoQO performance differs significantly between all regions (North Sea versus each of the others Chisquare statistics $<0.001$; Faroe-Iceland $\mathrm{P}=0.038$; Faroe-Canada $\mathrm{P}<0.001$ and Iceland-Canada $\mathrm{P}=0.019$ ).

Industrial plastics were relatively uncommon in stomachs of Icelandic fulmars (5\% of overall plastic mass) compared to industrial plastics in fulmars from the North Sea $(20 \%$ of overall plastic mass). This fits a pattern where industrial plastics seem more uncommon in fulmars from arctic Canada (Mallory et al. 2006; Mallory 2008), which suggests that the pelagic distribution of these granules is more strongly linked to industrial point sources than consumer wastes. This is the first assessment of abundance of plastic debris in stomachs of Icelandic fulmars. Data confirms that plastic pollution levels in the North Atlantic tend to decrease towards higher latitudes. Levels of pollution thus appear to link to regions of intense human coastal and marine activities, suggesting substantial current inputs in those areas. 


\section{Acknowledgements}

We want to thank the Natural History Museum in Bolungarvík (Iceland) for the provision of their facilities, the Icelandic fishermen, Marlous Heemstra, Therese S. Løkken, Job ten Horn, André Meijboom and Arjen Strijkstra for their support and help. We thank Elisa Bravo Rebolledo for data contributions for the Faroe Islands. Geert Aarts advised on the statistics to regional differences. 
$\lim -1=$

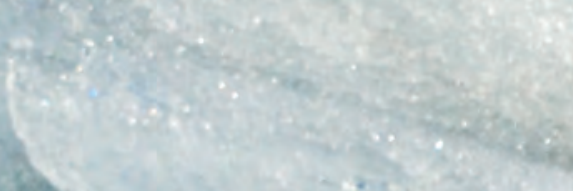

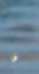


chapter 3

Elevated levels of ingested plastic in a high Arctic seabird, the northern fulmar (Fulmarus glacialis)

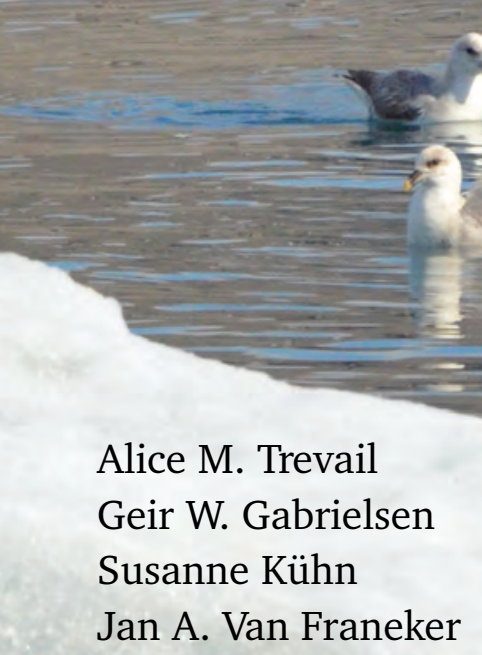




\begin{abstract}
Plastic pollution is of worldwide concern; however, increases in international commercial activity in the Arctic are occurring without the knowledge of the existing threat posed to the local marine environment by plastic litter. Here, we quantify plastic ingestion by northern fulmars, Fulmarus glacialis, from Svalbard, at the gateway to future shipping routes in the high Arctic. Plastic ingestion by Svalbard fulmars does not follow the established decreasing trend away from human marine impact. Of 40 sampled individuals, 35 fulmars (87.5\%) had plastic in their stomachs, averaging at $0.08 \mathrm{~g}$ or 15.3 pieces per individual. Plastic ingestion levels on Svalbard exceed the ecological quality objective defined by OSPAR for European seas. This highlights an urgent need for mitigation of plastic pollution in the Arctic as well as international regulation of future commercial activity.
\end{abstract}




\section{Introduction}

The threat of pollution in the Arctic is rising as commercial activity (such as shipping) increases, enabled by rapid sea ice decline (Kerr 2012) and driven by global politics and economic demand (Brigham 2011). The Arctic is currently an area of relatively low direct human impact (Halpern et al. 2008); however, increased shipping may put areas of high biodiversity at risk (Humphries \& Huettmann 2014). Indeed, shipping density has previously been linked to the prevalence of plastic ingestion by marine life as well as concentration of coastal human populations (Van Franeker et al. 2011; Kühn \& Van Franeker 2012). The global plastic industry is continuously expanding (PlasticsEurope 2013), the use of disposable plastic products persists (WRAP 2014), and it is likely that the already significant amounts of plastic litter entering the marine environment will increase (Law \& Thompson 2014). Latest estimates suggest that globally there is a minimum of 5.25 trillion pieces of plastic weighing 268,940 t floating on the ocean surface alone (Eriksen et al. 2014). The deleterious impacts of plastic litter are numerous, including transport of pollutants (Zarfl \& Matthies 2010) and invasive species (Barnes 2002), entanglement with and ingestion by marine fauna (Laist 1997), as well as economic costs (Mouat et al. 2010; Leggett et al. 2014). Considering this, there is an urgent need for a quantitative assessment of plastic pollution levels in the Arctic. Such an assessment can provide information for the development of international regulation to protect the marine environment for the future (Brigham 2011), as well as a tool for monitoring potential impacts of future commercial activity. Plastic ingestion has been documented in over 100 species of seabird (Laist 1997). This has led to the identification of species with characteristics that make them suitable as biological monitors of trends in plastic pollution (Van Franeker 1985; Van Franeker et al. 2011). Northern fulmars (Fulmarus glacialis) have been extensively used as an indicator species for plastic pollution levels in the Northern Hemisphere since they were first used for monitoring around the North Sea in the 1980s (Van Franeker et al. 2011). At present, data exist for much of the North-East Atlantic (Van Franeker et al. 2011; Kühn \& Van Franeker 2012), the Canadian Arctic (Provencher et al. 2009) and the eastern North Pacific (Avery-Gomm et al. 2012; Donnelly-Greenan et al. 2014). Northern fulmars are entirely oceanic feeders (Weimerskirch et al. 2001; Garthe et al. 2004), and their omnivorous foraging behaviour (Hamer et al. 1997) renders them particularly vulnerable to plastic ingestion (Van Franeker et al. 2011). Hard prey items, including plastic, remain in a fulmar's muscular stomach until they are broken down to a size that can pass through the gut, within approximately 1 month (Van Franeker et al. 2011). Therefore, stomach plastic contents represent a recent period prior to death, and thus plastic pollution in their foraging area (Van Franeker et al. 2011). Within Europe, northern fulmars are defined by the Oslo-Paris Convention (OSPAR) for the North-East Atlantic as an indicator species of plastic pollution (OSPAR 2008). OSPAR recommendations state that for acceptable ecological quality (EcoQO), $<10 \%$ of the monitored population of northern fulmars should have more than $0.1 \mathrm{~g}$ of plastic in the stomach (OSPAR 2008). Levels of plastic pollution typically decrease away from areas of high human impact and commercial activity, thereby 
often decreasing towards the poles (Barnes 2002; Barnes 2005; Kühn \& Van Franeker 2012). This decrease can be attributed to the main sources of plastic to the ocean, including accidental losses during transport, irresponsible human behaviour, improper waste management and loss during natural disasters. However, there is no complete or recent information regarding plastic ingestion by northern fulmars at the highest breeding latitudes in Europe. Svalbard, in the European Arctic, is an area of high seabird biodiversity (Humphries \& Huettmann 2014) where there will likely be a substantial increase in shipping traffic in the years to come (Smith \& Stephenson 2013) and therefore potential increases in plastic pollution. Although the Arctic has long lost its wilderness status (France 1992), measurements of the extent of anthropogenic litter in the European Arctic only exist for the seafloor (Bergmann \& Klages 2012). To assess plastic pollution levels in the European Arctic, this study quantified the amount of plastic ingested by northern fulmars from Spitsbergen, the largest island of the Svalbard archipelago. In the 1980s, plastic ingestion by fulmars from Spitsbergen and Bear Island (mid-way between Spitsbergen and mainland Norway) was observed during diet studies (Gjertz et al. 1985; Lydersen et al. 1985; Van Franeker 1985; Lydersen et al. 1989). However, the mass of plastic was not recorded and data from Spitsbergen are incomplete; therefore, a temporal comparison with 1980s data is not possible. This study will be the first dedicated study of plastic ingestion by arctic fulmars in this area, and thus represents a valuable northwards expansion of ongoing Atlantic/North Sea monitoring efforts. This paper presents the results from Svalbard, alongside analysis of spatial trends.

\section{Materials and Methods}

This project is registered in the 'Research in Svalbard' (RiS) database, within the Svalbard Science Forum (Project ID: 6355). Permission to sample fulmars was granted by Sysselmannen, the governing body on Svalbard. A total of 40 fulmars were shot outside of the breeding season for a broad range of research purposes, in collaboration with other research groups. This method was selected because of the absence of longline fisheries in the Svalbard area, from which it would otherwise be possible to collect bycatch individuals (e.g. Kühn \& Van Franeker 2012). In addition, collecting beached individuals is infeasible because of rapid scavenging (e.g. by polar foxes, arctic skuas, great skuas, glaucous gulls) and inaccessibility of beaches. Furthermore, stomach flushing alone would likely not give a full representation of stomach contents, as many of the hard items would remain in the gizzard: the muscular section of the stomach. A sample size of 40 has been recommended to quantify plastic ingestion with statistical confidence in fulmars (Van Franeker \& Meijboom 2002).

Sampling was carried out in accordance with high EU standards and best professional practices, and all efforts were made to minimise suffering. Collaboration with other studies (e.g. samples for diet studies, stable isotope analysis studies, tissue contaminant studies for POPs and heavy metals by Norwegian scientists, and tissue radionuclide studies 
by Japanese scientists investigating the fallout from Fukushima 2011) ensured maximum sampling from killed individuals.

Fulmars were sampled in Isfjord, Svalbard $\left(78.3^{\circ} \mathrm{N}, 16.1^{\circ} \mathrm{E}\right)$, from 21 to 23 September 2013. Dissections were undertaken at the University Centre in Svalbard (UNIS), Longyearbyen, following the protocol used by the North Sea monitoring programme (Van Franeker 2004) to determine age and sex as well as morphological characteristics. Stomachs were collected whole, and both the proventriculus and gizzard were rinsed over a 1-mm sieve.

Stomach plastic contents were characterised and quantified with IMARES (Texel, Netherlands) according to the North Sea monitoring protocol (Van Franeker et al. 2011): plastic pieces were counted and weighed by category once dry on an electronic Sartorius scale accurate to $0.0001 \mathrm{~g}$. Plastics were sorted visually into industrial plastics: raw plastic pellets produced by plastic manufacturers, and user plastics: all forms of plastic used by consumers, such as fragments of hard plastics, sheets, threads or foams. Data were compared to other regions where plastic loading in northern fulmar stomachs has been monitored. Data were provided from Jan Van Franeker for the most recent 5-year period (2007-2011) in the English Channel, the North Sea (comprising of East England, the Scottish Islands, Belgium, Germany, the Netherlands, Denmark and the North Sea coasts of Sweden and South Norway) and the Faroe Islands, published within the 'Save the North Sea' monitoring work (Van Franeker \& SNS Fulmar Study Group 2013) as well as for Iceland for 2011 (Kühn \& Van Franeker 2012). Summary data for Arctic Canada were also used for comparison (Mallory et al. 2006; Mallory 2008; Provencher et al. 2009).

All data analyses were carried out using $\mathrm{R}$ version 3.1.0. Regional averages are presented as the arithmetic mean (unless otherwise stated) using all individuals, including those with no ingested plastic. Data were not normally distributed before or after relevant transformation (Shapiro-Wilk $\mathrm{P}<0.05$ ); therefore, nonparametric tests (Mann-Whitney U test or Kruskal-Wallis) were used to compare regional differences in plastic ingestion. In addition, the geometric mean and OSPAR EcoQO performance were calculated to minimise the effect of outliers, as per previous monitoring work (Kühn \& Van Franeker 2012).

\section{Results}

As determined from dissections (Van Franeker 2004), 5 out of the 40 fulmars were adults, 7 were second-year birds (i.e. chicks of 2012), and the remainder were sub-adults (ca. 3-5 or more years old, having never bred before). None of the fulmars sampled in September had bred during the summer of 2013, determined by the lack of remnants of a brood patch. Sexes were equally represented with 21 females and 19 males. All birds were of the arctic 'coloured' type (colour phases L, D and DD as in Van Franeker (2004)). No fulmars were of the light plumage colour that dominates in colonies south of the Arctic. 


\section{Plastic ingestion by fulmars on Svalbard}

On Svalbard, 35 fulmars (87.5\%) of the 40 sampled individuals contained plastic (i.e. the incidence rate), equating to an average of 15.3 pieces $( \pm$ s.e. $=5.5, n=40$ ) of plastic per individual or an average total mass of $0.08 \mathrm{~g}( \pm 0.02 \mathrm{~g}$ ) per individual. Full details of stomach plastic are given in Tables 3.1 and 3.2. The maximum stomach plastic contents by both number and weight were recorded in the same individual: 200 pieces, weighing $0.4990 \mathrm{~g}$. Industrial plastic pellets made up on average $10.8 \%( \pm 4.5 \%)$ of the mass of all stomach plastic content in individual fulmars; the remainder of which was user plastic. An example of stomach plastic content is given in Figure 3.1. In this study we found that on Svalbard, $22.5 \%$ of northern fulmars have $\geq 0.1 \mathrm{~g}$ of plastic in their stomach, which exceeds the level defined by OSPAR as the Ecological Quality Objective for the North Sea (EcoQO; 10\%). The amount of plastic in stomachs of fulmars in this study did not differ between male and female birds in terms of both mass ingested and number of pieces (Mann-Whitney U test, $\mathrm{P}>0.05$ ). Similarly, we found no statistical difference in stomach plastic content between the different age groups sampled in this study (Mann-Whitney $\mathrm{U}$ test, $\mathrm{P}>0.05$ ) or by the different colour morphs of fulmars (Mann-Whitney U test, $\mathrm{P}>0.05)$.

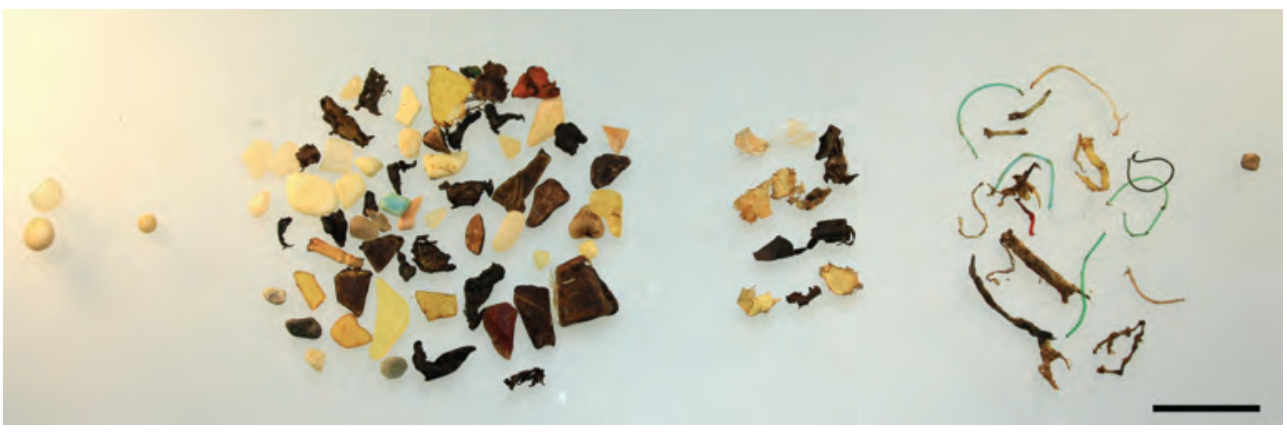

Figure 3.1. Stomach plastic contents of an individual northern fulmar from Svalbard, 2013. L-R: Industrial pellets; probably industrial; fragments; sheets; threads; foam. Scale bar indicates $1 \mathrm{~cm}$.

Table 3.1. Plastic ingested by northern fulmars from Svalbard, according to age of individual. Table presents number of pieces per individual (n), grams per individual $(\mathrm{g})$, and the maximum grams in an individual within each age class $(\max g$ ). \pm se shows standard error. EcoQO performance gives the percentage of the population with more than $0.1 \mathrm{~g}$ of ingested plastic.

\begin{tabular}{lrcccccccc} 
& $\begin{array}{c}\text { sample } \\
\text { size }\end{array}$ & Incidence & $\mathbf{n}$ & $\mathbf{\pm s e}$ & $\mathbf{g}$ & $\mathbf{\pm s e}$ & Max $\mathbf{g}$ & $\begin{array}{c}\text { Geometric } \\
\text { mass }\end{array}$ & $\begin{array}{c}\text { EcoQO } \\
\text { performance }\end{array}$ \\
\hline ALL & 40 & $87.5 \%$ & 15.32 & 5.51 & 0.08 & 0.02 & 0.50 & 0.023 & $22.5 \%$ \\
Adults & 5 & $80.0 \%$ & 4.20 & 2.52 & 0.04 & 0.02 & 0.12 & 0.013 & $20.0 \%$ \\
Non-adults & 35 & $89.0 \%$ & 16.91 & 6.25 & 0.09 & 0.02 & 0.50 & 0.025 & $22.9 \%$
\end{tabular}




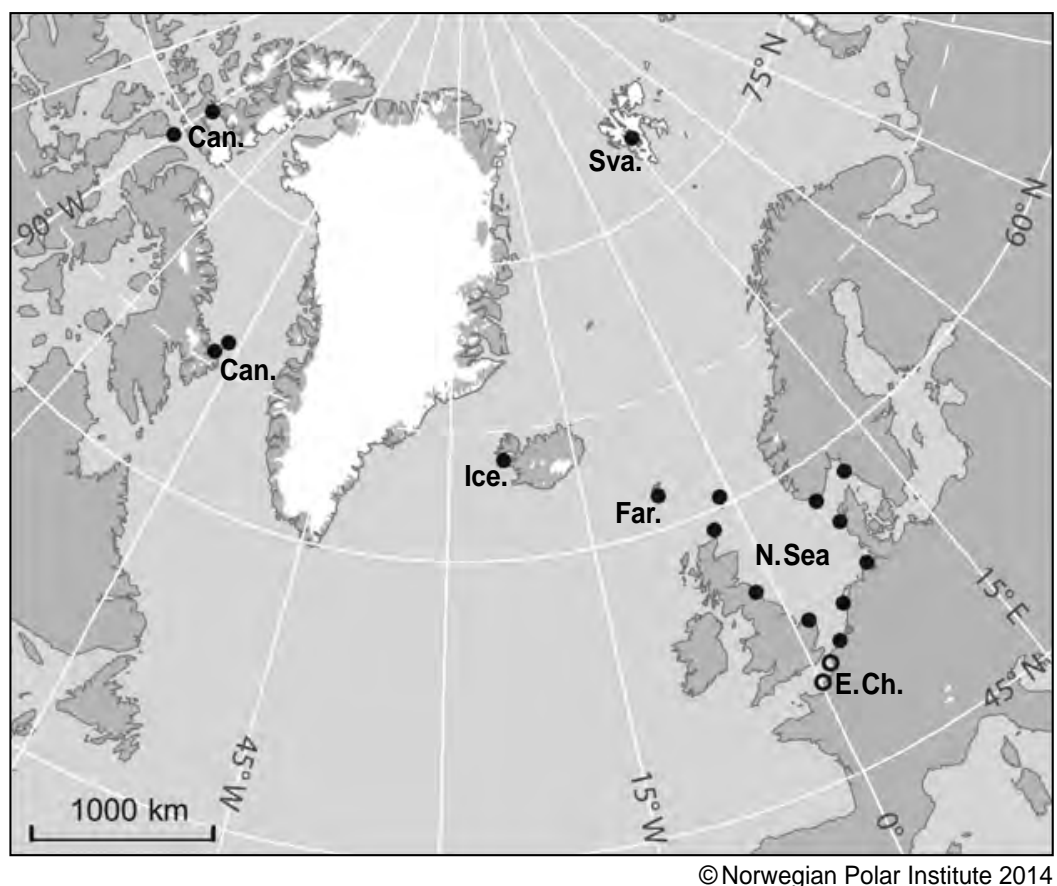

Figure 3.2. Map showing all study locations used for regional comparison of plastic ingestion by northern fulmars: Svalbard (Sva.), Arctic Canada (Can.), Iceland (Ice.), Faroe Islands (Far.), the North Sea (N. Sea) and the English Channel (E. Ch.; hollow circles). White shading indicates ice cover. Dashed line gives limit of the Arctic Circle around $66^{\circ} 33^{\prime} 44^{\prime \prime} \mathrm{N}$.

Table 3.2. Plastic ingested by northern fulmars from Svalbard ( $n=40)$, according to type of plastic. Table presents number of pieces per individual (n), grams per individual (g), and the maximum grams in an individual within each age class $(\max g$ ). \pm se shows standard error. Maximum stomach content is by a single individual.

\begin{tabular}{lccccccc} 
& Incidence & $\mathbf{n}$ & $\mathbf{s e}$ & $\mathbf{g}$ & $\mathbf{\pm s e}$ & $\mathbf{m a x} \mathbf{g}$ & $\begin{array}{c}\text { geometric } \\
\text { mass }\end{array}$ \\
\hline ALL PLASTICS & $87.5 \%$ & 15.32 & 5.51 & 0.080 & 0.02 & 0.499 & 0.023 \\
$\begin{array}{l}\text { Industrial plastic } \\
\text { User plastic }\end{array}$ & $23 \%$ & 0.45 & 0.17 & 0.006 & 0.00 & 0.051 & 0.001 \\
sheetlike & $83 \%$ & 14.88 & 5.41 & 0.074 & 0.02 & 0.490 & 0.018 \\
threadlike & $35 \%$ & 1.53 & 0.53 & 0.004 & 0.00 & 0.071 & 0.001 \\
foamed & $45 \%$ & 1.90 & 0.61 & 0.018 & 0.01 & 0.318 & 0.002 \\
fragments & $10 \%$ & 0.68 & 0.45 & 0.000 & 0.00 & 0.008 & 0.000 \\
other & $80 \%$ & 10.72 & 4.60 & 0.049 & 0.02 & 0.480 & 0.013 \\
& $5 \%$ & 0.05 & 0.03 & 0.003 & 0.00 & 0.082 & 0.000
\end{tabular}



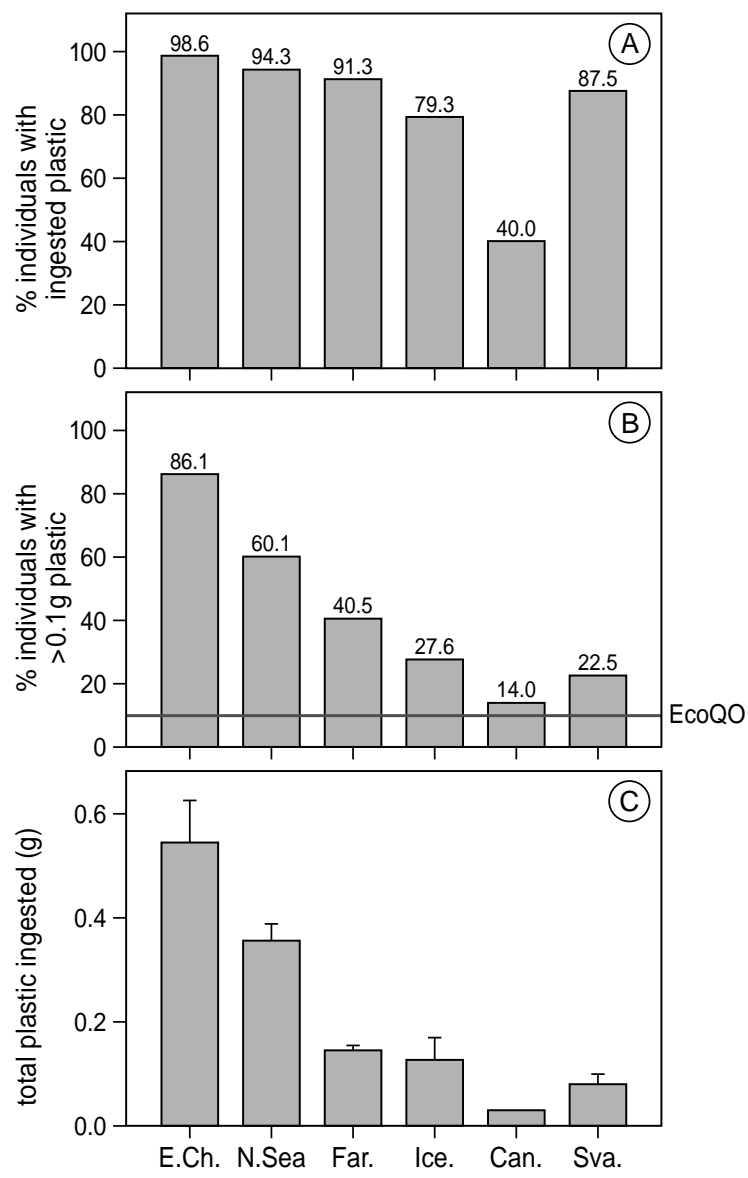

Figure 3.3. Regional differences in plastic ingestion by northern fulmars in the North Atlantic, according to (A) population incidence of plastic ingestion, (B) population incidence of over 0.1 gram of plastic, dashed line shows the Ecological Quality Objective (EcoQO) defined by OSPAR, and (C) arithmetic mean mass, error bars show standard error. Data have been collated for the English Channel (E.Ch., 2007-2011, $\mathrm{n}=72$ ), the North Sea (N. Sea, 2007-2011, $\mathrm{n}=58$ ), the Faroe Islands (Far., 2007-2011, $\mathrm{n}=699$ ) (Van Franeker and SNS Fulmar Study Group 2013), Iceland (Ice., 2011, $n=58$ ) (Kühn and Van Franeker 2012), Svalbard (Sva., 2013, $\mathrm{n}=40$ ) (this study) and Arctic Canada (Can., 2002-2009, $\mathrm{n}=169$ ) (Mallory et al. 2006; Mallory 2008; Provencher et al. 2009).

\section{Latitudinal comparison}

Plastic content of fulmar stomachs from Svalbard was compared to monitoring data from multiple regions in the North-East Atlantic (Figure 3.2). Overall, incidence of stomach plastic differs significantly between study regions in the North Atlantic (mass and number of pieces; Kruskal- Wallis, $\mathrm{P}<0.05$ ).

From the English Channel north to Arctic Canada, there is a decrease in stomach plastic incidence and mass with latitude (Figure 3.3). However, plastic content (incidence and 
mass) is greater on Svalbard than at lower latitudes in Arctic Canada. In addition, there is no difference in the amount of plastic (mass and number of pieces) in stomachs of northern fulmars from Svalbard and Iceland, (Mann- Whitney U test, P > 0.05). On Svalbard, the incidence of stomach plastic was higher than on Iceland (Figure 3.3A), although mass was similar in the two locations (Figure 3.3B-C). The geometric mean masses of stomach plastic on Iceland and Svalbard are 0.020 and $0.024 \mathrm{~g}$, respectively.

\section{Discussion}

This study has achieved a baseline value of plastic ingestion by fulmars that will facilitate the future detection of changes in marine plastic pollution and potential impacts of increased commercial activity.

Unfortunately, data for the 1980s (Gjertz et al. 1985; Lydersen et al. 1985; Van Franeker 1985; Lydersen et al. 1989) do not allow a proper analysis for possible changes over time in stomach plastic content. The data in these early publications give only the number of items and appear contradictory between information for Spitsbergen (29\% individuals with plastic $(\mathrm{n}=62)$, and an average of 0.75 pieces $(\mathrm{n}=20)$ in Gjertz et al. (1985), Lydersen et al. (1985) and Lydersen et al. (1989)) and nearby Bear Island (82\% individuals with plastic and an average of 4.5 pieces $(n=22)$ in Van Franeker (1985)). Furthermore, from North Sea fulmar data, it appears that sizes of plastic particles have changed over time: particles have become smaller (Van Franeker \& Meijboom 2002), with currently different number to mass ratios than in the 1980s, which complicates comparisons. As expected from North Sea data in (Van Franeker et al. 2011), the data in Table 3.1 do suggest differences between young and adult birds, but the small sample size for adults prevents robust statistical evaluation in this case. However, adults and non-adults are similar in EcoQO performance and allow combined further discussion in this baseline. Later studies need to provide more insight into potential age differences and their implications for interpretation of plastic monitoring data.

Plastic in stomachs of northern fulmars on Svalbard does not follow the established trend of a decrease with latitude or distance from human marine impact (using measure of impact from Halpern et al. (2008)). Instead, stomach plastic content of fulmars from Svalbard is higher than expected. This study reports the highest levels of plastic ingestion reported in an Arctic colony of northern fulmars. Incidence of stomach plastic and mass of stomach plastic are considerably higher than those recorded at lower latitudes in Arctic Canada (Mallory et al. 2006; Mallory 2008; Provencher et al. 2009). In addition, levels of stomach plastic on Svalbard are comparable to those on Iceland, approximately 2,000 km further south, contrary to the expected latitudinal decrease (Kühn \& Van Franeker 2012).

Although foraging distance of fulmars can be large (Edwards et al. 2013), it may be that the individuals sampled were exhibiting central-place foraging, as found in immature birds following the breeding period in other species (Votier et al. 2011; Riotte-Lambert \& Weimerskirch 2013). Indeed, sampled fulmars from Svalbard were all of the darker colour 
phase, of which very few (10\%) are found in the northern North Sea regions in the month prior to sampling (Van Franeker, unpublished). Those that are found in the northern North Sea are predominately younger still than the sample of fulmars in this study. It is therefore probable that the plastic in their stomachs was ingested locally, rather than in more polluted regions further south. If distance from human impact was the primary driver of plastic ingestion, as previously proposed (Kühn \& Van Franeker 2012), levels of plastic ingestion in fulmars from Svalbard would be expected to fall between those in Iceland and Arctic Canada. Indeed, as anticipated, levels on Svalbard are higher than Arctic Canada, where study sites are more remote from population centres. Likewise, compared to the North Sea region (Van Franeker et al. 2011), both lower stomach plastic amounts and higher proportions of user plastics on Svalbard reflect distance from industry and commercial shipping, in accordance with previous theory. However, Svalbard is more remote from human impact than Iceland (Halpern et al. 2008), and yet the amounts of plastic in stomachs of fulmars from the two locations are similar, indicating a need for alternative or additional hypotheses.

The cause of elevated levels of plastic ingestion on Svalbard is uncertain, and therefore, a key knowledge gap for future research has been identified. Transportation of plastic from outside of the Arctic by surface water currents is a likely explanation. Currents along the Norwegian coast may carry floating debris from the polluted North Sea up to the Barents Sea and Svalbard, thus increasing plastic ingestion despite the absence of dense population centres in the region. Van Sebille et al. (2012) hypothesised that converging water currents actually result in an oceanic gyre in the Barents Sea, where plastic litter would accumulate; however, this is yet to be proven. Alternatively, ingested plastic may originate in or around the Barents Sea, either from the Southern Barents Sea fishing fleet (Humphries \& Huettmann 2014) or potential release during periods of sea ice melt (Obbard et al. 2014). To confirm or reject these hypotheses would be a useful study for the future and will help to identify how to mitigate plastic in the Arctic. The high levels of stomach plastic content observed in Svalbard not only highlight the risk to seabirds from plastic pollution, but may also be a considered as a general warning of effects of plastic litter in the Arctic. Floating plastic debris may act as a transport vector to the Arctic for both pollutants (Zarfl \& Matthies 2010) and invasive species (Barnes 2002)—both may act as important stressors with threats to biodiversity, particularly under climate warming scenarios (Serreze et al. 2007). Compounds within the plastics may have negative consequences on both wildlife and human health in the region (Oehlmann et al. 2009). High prevalence of plastic litter in the Arctic, outside of territorial waters, emphasises the need for international mitigation of plastic litter at source, as well as strict enforcement of legislation for commercial activity in the region.

\section{Conclusions}

This study provides a valuable baseline for plastic litter needed to monitor the future impacts of commercial activity in the Arctic. Furthermore, high levels of plastic ingestion in a seabird breeding area at great distance from human impact highlight the need for urgent 
mitigation of plastic pollution in the Arctic as well as implementation of strict regulation for future commercial activity.

\section{Acknowledgements}

This work was funded in full by Svalbard Miljøvernfond and the Nordic Council. Thanks to participants in the Save the North Sea monitoring projects for providing data shown in Figure 3.3. Many thanks also to anonymous reviewers for improvements to the manuscript. 


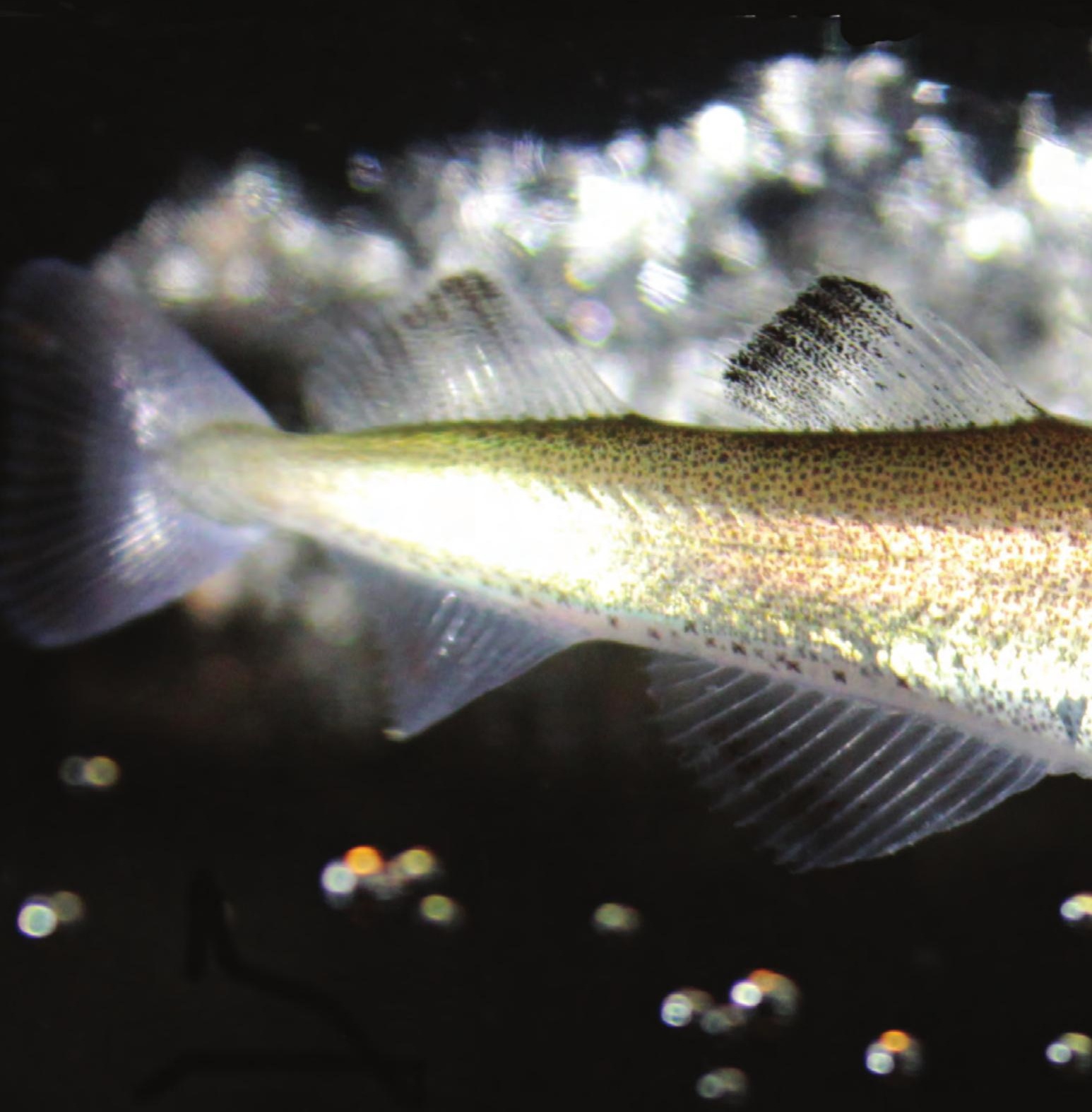



(Boreogadus saida) in the Arctic Ocean

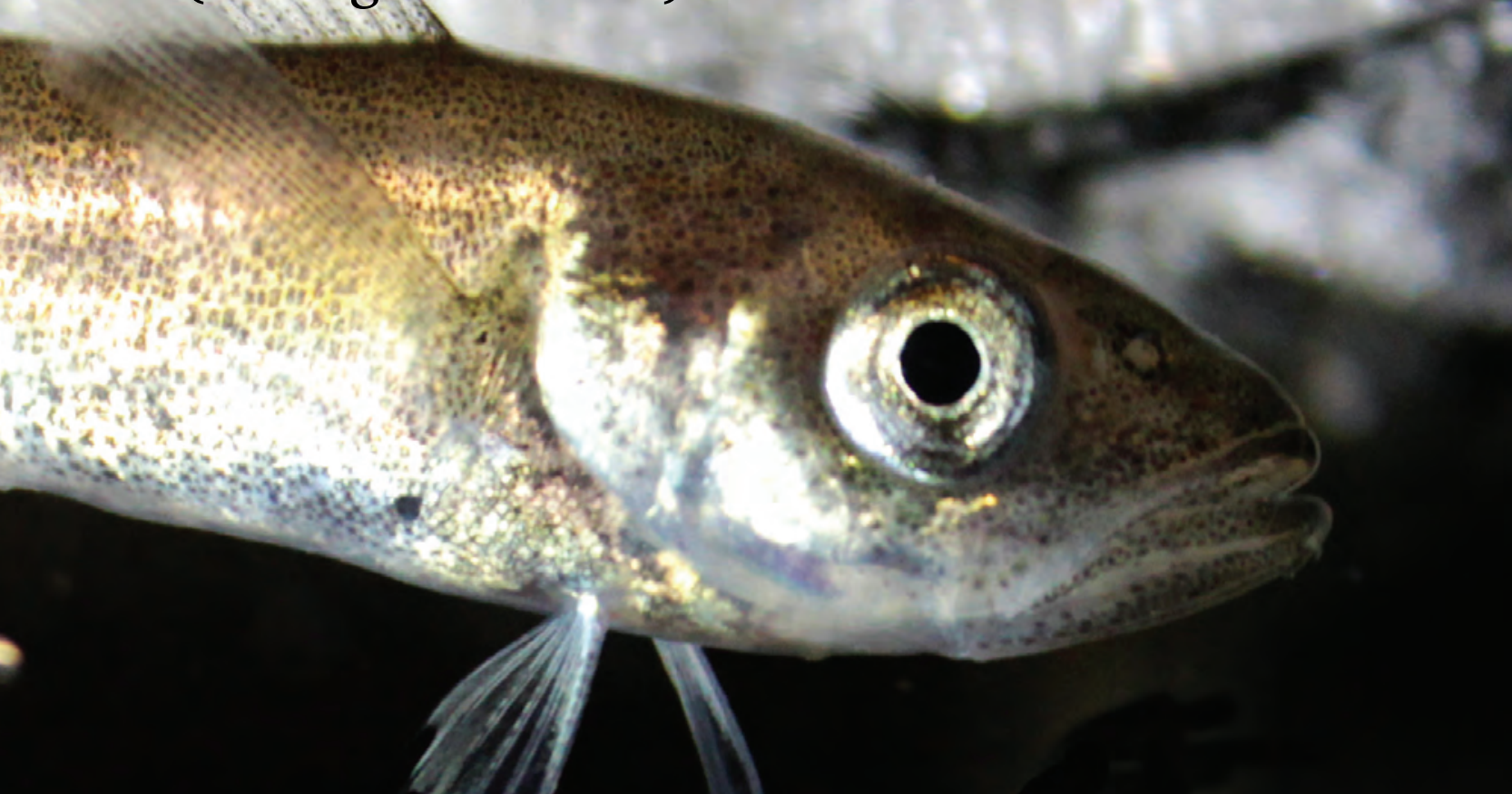

Susanne Kühn, Fokje L. Schaafsma, Bernike van Werven, Hauke Flores, Melanie Bergmann, Marion Egelkraut-Holtus, Mine B. Tekman, Jan A. van Franeker

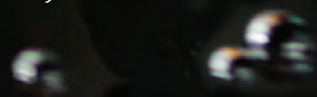




\begin{abstract}
One of the recently recognised stressors in Arctic ecosystems concerns plastic litter. In this study, juvenile polar cod (Boreogadus saida) were investigated for the presence of plastics in their stomachs. Polar cod is considered a key species in the Arctic ecosystem. The fish were collected both directly from underneath the sea ice in the Eurasian Basin and in open waters around Svalbard. We analysed the stomachs of 72 individuals under a stereo microscope. Two stomachs contained non-fibrous microplastic particles. According to $\mu$ FTIR analysis, the particles consisted of epoxy resin and a mix of Kaolin with polymethylmethacrylate (PMMA). Fibrous objects were excluded from this analysis to avoid bias due to contamination with airborne micro-fibres. A systematic investigation of the risk for secondary micro-fibre contamination during analytical procedures showed that precautionary measures in all procedural steps are critical. Based on the two non-fibrous objects found in polar cod stomachs, our results show that ingestion of microplastic particles by this ecologically important fish species is possible. With increasing human activity, plastic ingestion may act as an increasing stressor on polar cod in combination with ocean warming and sea-ice decline in peripheral regions of the Arctic Ocean. To fully assess the significance of this stressor and its spatial and temporal variability, future studies must apply a rigorous approach to avoid secondary pollution.
\end{abstract}




\section{Introduction}

Debris ingestion by a wide range of marine organisms has been demonstrated in various studies from all over the world. At least 331 marine species have been documented to ingest plastic between the 1960s and 2015 of which 92 were fish species (Kühn et al. 2015). However, this is a rap- idly developing field and the online database 'Litterbase' (Bergmann et al. 2017b) currently holds 168 fish species. The commercial value and worldwide consumption of fish have triggered an interest to study the abundance of plastic in fish, as it raises concerns about human exposure (Rochman 2015).

Marine debris and in particular plastics have been found in all ocean basins of the world (Barnes et al. 2009; Eriksen et al. 2014; Galgani et al. 2015; van Sebille et al. 2015). The Arctic region has long been considered a pristine environment, relatively undisturbed by humans. However, recent studies have shown that plastic debris has reached the Arctic oceanic and sea-ice environments, and its wildlife (Schulz et al. 2010; Obbard et al. 2014; Lusher et al. 2015; Trevail et al. 2015b; Bergmann et al. 2017a; Bergmann et al. 2017c; Buhl-Mortensen \& Buhl-Mortensen 2017; Cózar et al. 2017). A suggested presence of an accumulation area in the Barents Sea (van Sebille et al. 2015) was supported by Cózar et al. (2017) with recent field data and additional modelling indicating a peak accumulation of plastic in the vicinity of Svalbard and Novaya Zemlya. Microplastics were recorded from Arctic sea-ice cores (Obbard et al. 2014; Peeken et al. 2018) and in Arctic surface waters where levels of microplastic pollution fell in the range of those in other areas in the North Atlantic and the North Pacific (Lusher et al. 2015).

The ingestion of plastic debris by Arctic marine species has been recorded in organisms ranging from marine mammals (Martin \& Clarke 1986; Finley 2001) to seabirds (Lydersen et al. 1989; Mallory 2008; Provencher et al. 2010; Trevail et al. 2015a) and blue mussels (Mytilus spp.; Lusher et al. 2017a). However, to our knowledge there are hitherto only two reports of litter ingestion by an Arctic fish species, the Greenland shark (Somniosus microcephalus; Leclerc et al. 2012; Nielsen et al. 2013). The incidence of plastic ingestion in Arctic food webs is likely to increase as plastic pollution rises in the Arctic (Tekman et al. 2017). The ecological consequences of plastic ingestion are currently largely unknown. Large items may get stuck in organisms and obstruct the intestinal tract, and may cause injury or a false sense of satiation (Kühn et al. 2015); very small particles may translocate and pass to organs or cell with unknown consequences (Jani et al. 1992; Browne et al. 2008; Brennecke et al. 2016). Although not covered in this study, the recent detection of various persistent organic pollutants adsorbed to passive polyethylene samplers deployed west of Svalbard (Sun et al. 2016) highlights the potential of transfer of toxins upon ingestion by (Arctic) organisms (Tanaka et al. 2015; Chen et al. 2018).

Polar cod (Boreogadus saida) is regarded as a key species in the Arctic food web because it is regularly consumed by top predators (Lønne \& Gabrielsen 1992; Mehlum \& Gabrielsen 1993; Weslawski et al. 1994; Hop \& Gjøsæter 2013) and because of its high energetic value (Hop \& Gjøsæter 2013; David et al. 2015). They occur in large numbers directly underneath the Arctic sea ice (Lønne \& Gulliksen 1989; Gradinger \& Bluhm 2004; 
David et al. 2015). Young polar cod are strongly associated with the sea-ice habitat (Gradinger \& Bluhm 2004; David et al. 2015; Kohlbach et al. 2017), as ice-associated amphipods and copepods are its main prey (Lønne \& Gulliksen 1989; Kohlbach et al. 2017). In the under-ice layer, elevated levels of microplastics have been reported (Obbard et al. 2014; Peeken et al. 2018). The under-ice layer could thus be a zone of high plastic litter concentration and a source of plastics for organisms such as polar cod foraging in the under-ice environment. For this study, juvenile polar cod were investigated for the ingestion of plastics in order to provide a first baseline of marine plastic litter ingestion by a key fish species in the Arctic food web which forages specifically in the under-ice habitat.

\section{Methods}

\section{Sampling}

Samples of polar cod were collected during three research cruises in the Arctic between 2012 and 2015 (David et al. 2015; Mark 2015; Flores et al. 2016). Details of each cruise such as ship, date and fishing method are presented in Table 4.1. Sample locations are presented in Figure 4.1. All individuals from PS80 and PS92 were collected in ice covered waters, while fish from the HE 451.1 expedition were caught in open water.

During the expeditions PS80 and PS92 with the ice breaker RV Polarstern, fish were caught along the under-ice surface (0-2 m depth) using Surface and Under Ice Trawls (SUIT). SUIT was designed to sample the upper two metres of the water column either in open water or directly beneath the usually hardly accessible sea ice (Van Franeker et al. 2009; Flores et al. 2012). Half hour trawls were conducted at speeds between 2 and 3 knots. SUIT consists of a steel frame with a $2 \times 2 \mathrm{~m}$ opening, and two nets with different mesh sizes attached (a $0.3 \mathrm{~mm}$ mesh and a $7 \mathrm{~mm}$ half-mesh). Floats attached to the frame ensure that the trawl stays at the surface or the sea-ice underside. A bridle connected to one side of the SUIT frame forces the trawl to shear out sideways, away from the wake of

Table 4.1. Details on the three research cruises where polar cod (Boreogadus saida) used for stomach content analysis was collected.

\begin{tabular}{|c|c|c|c|c|c|c|}
\hline $\begin{array}{l}\text { Expedition } \\
\text { name }\end{array}$ & $\begin{array}{l}\text { Expedition } \\
\text { number }\end{array}$ & $\begin{array}{c}\text { Research } \\
\text { vessel }\end{array}$ & Area & Time & Fishing gear & $\begin{array}{l}\text { Number of } \\
\text { stations }\end{array}$ \\
\hline IceARC & PS80 & Polarstern & Eurasian Basin & $\begin{array}{c}\text { August - } \\
\text { October } 2012\end{array}$ & SUIT & 11 \\
\hline \multirow[t]{2}{*}{ TRANSSIZ } & PS92 & Polarstern & $\begin{array}{l}\text { Svalbard Shelf, } \\
\text { Yermak Plateau }\end{array}$ & June 2015 & $\begin{array}{c}\text { SUIT } \\
\text { (+ RMT } 1 \text { ind.) }\end{array}$ & 5 \\
\hline & HE451.1 & Heincke & $\begin{array}{l}\text { Svalbard: } \\
\text { Kongsfjorden, } \\
\text { Billefjorden }\end{array}$ & $\begin{array}{c}\text { September } \\
2015\end{array}$ & $\begin{array}{l}\text { Juvenile } \\
\text { fish trawl }\end{array}$ & 2 \\
\hline
\end{tabular}




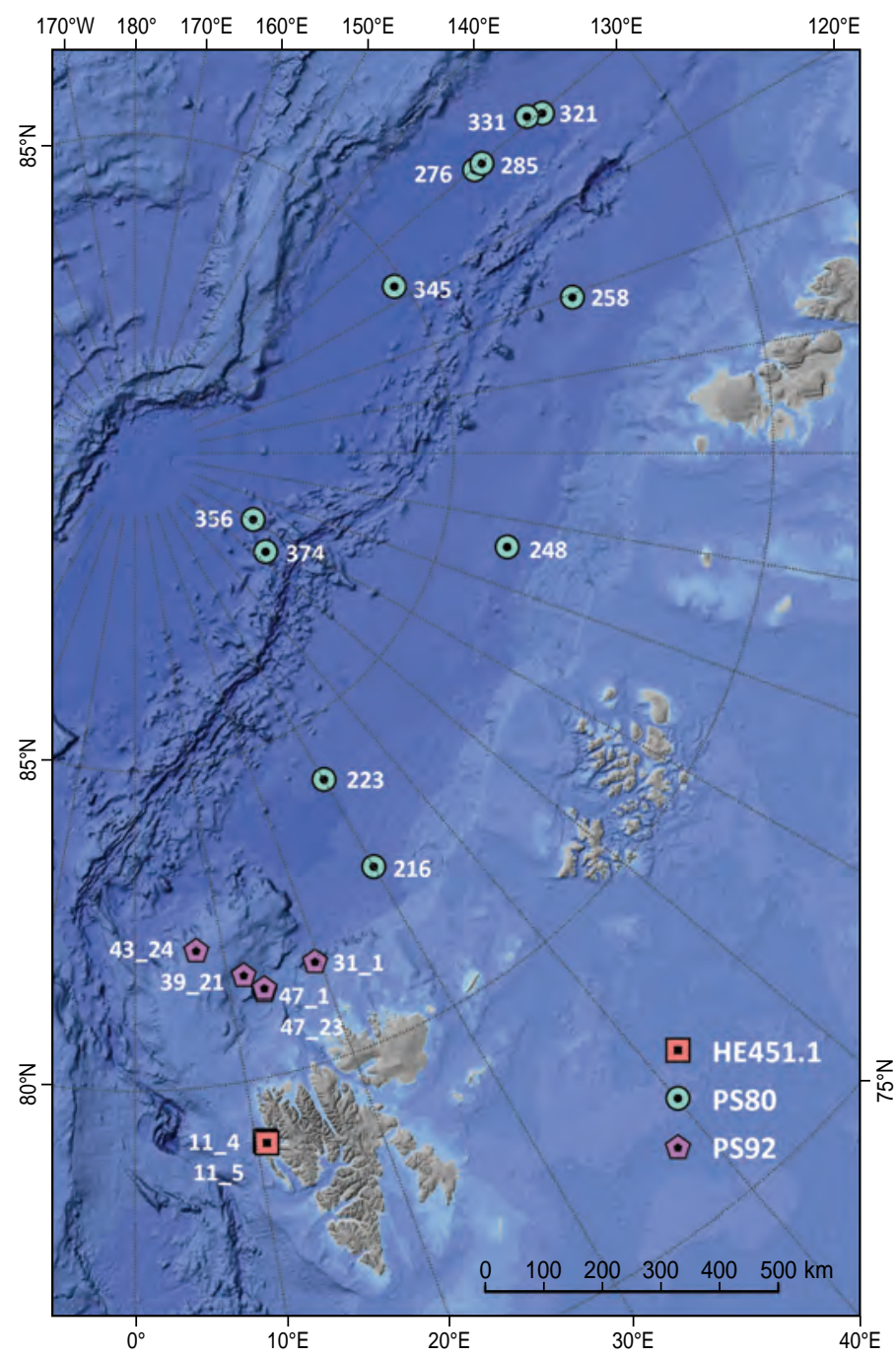

Figure 4.1. Map of sample stations for polar cod (Boreogadus saida) from three different research expeditions (HE451.1, squares; PS80, circles and PS92, pentagons). Numbers indicate stations where polar cod was caught (For details see Online Resource 2).

the ship, ensuring sampling underneath relatively undisturbed ice floes. A detailed description of SUIT sampling during PS80 is provided by David et al. (2015). One fish from PS92 was collected with a Rectangular Midwater Trawl (RMT) between 0 and $50 \mathrm{~m}$ depth. Polar cod from open water near Svalbard was caught with a juvenile fish trawl at depths between 13 and $30 \mathrm{~m}$ towed at 2.7-3.3 kn for $15 \mathrm{~min}$ at depths between 13 and 30 m. To effectively catch small and juvenile fish and surface them alive, a fish-lift (Holst \& McDonald 2000) connected to the juvenile fish trawl was used. 


\section{Stomach content analysis}

In total, 72 individual polar cod were used for the purpose of this study. The total length, weight and sex of each individual were recorded. Although the initial reason for stomach analyses was an assessment of diet of the fishes, the samples were also used to assess plastic ingestion. The stomachs were dissected from the fish using scissors, which took place either on board or later in the laboratory. The stomach contents were removed from the stomachs by cutting them open and rinsing out the content into a clean petri dish using deionised water. All stomachs and extracted stomach contents were stored in $4 \%$ hexamine- buffered formaldehyde-sea water solution. In order to remove the formaldehyde solution, the stomach contents were rinsed for a few minutes by placing the sample on a $35 \mu \mathrm{m}$ sieve under a running tap in a fume hood prior to the plastic analysis.

After carefully pouring the stomach content in a clean Bogorov counting chamber, samples were checked visually for plastics, using a Discovery V8 stereomicroscope (Zeiss, Germany). Suspect items were photographed and measured using an AxioCam MRc with AxioVision40 V4.8.2.0 software (Zeiss, Germany) and collected for later analysis. All suspect particles except micro-fibres were analysed by $\mu$ FTIR (Shimadzu FTIR IRTracer100, Infrared Microscope AIM-9000, diamond cell (DC-3; Specac)) to confirm whether a particle was of synthetic origin and to identify the polymer type. Spectra were measured in transmission mode on different points of the sample to avoid disturbance by surface fouling on the particles. Several reference libraries containing about 14,500 spectra in total were used to compare the detected spectra (Shimadzu Libraries, ST Japan-Europe, standard data base from Biorad Sadtler and other libraries).

\section{Airborne fibre contamination}

In this study and most other microplastic publications (e.g. Foekema et al. 2013; Rummel et al. 2016; Hermsen et al. 2017), the term 'fibres' refers to 'micro-fibres', the omnipresent dust-like bits from clothing, carpets or other woven garments. Micro-fibres in the marine environment are assumed to reach the oceans via sewage facilities (Browne et al. 2011) or atmospheric distribution (Dris et al. 2016). More sturdy 'fibres' that would not become airborne such as those derived from, e.g. multifilament ropes, network and fishing line, are addressed as 'threadlike' materials.

Secondary contamination of samples through airborne micro-fibre dust has been observed as a serious problem in earlier studies (e.g. Davison \& Asch 2011; Foekema et al. 2013; Rummel et al. 2016). Wesch et al. (2017) even showed that such secondary fibre contamination is basically unavoidable in nearly any type of sampling and laboratory setting, and seriously affects results. For this reason, our primary goal was thus to quantify ingestion of non-fibrous plastic particles that are not subject to such airborne bias. Nonetheless, as the main type of plastics reported in the ice cores of Obbard et al. (2014) concerned micro-fibres and these micro-fibres are often used in studies to show a human impact on marine environments, we made all efforts to quantify and compare fibre abundance in our samples and controls. 
The fish used for this diet study were, however, dissected without a specific protocol to avoid secondary pollution, and it is unclear how many airborne fibres might have polluted the samples before their processing in the plastic study. In spite of this caveat, the use of different handling protocols does provide an opportunity to investigate the effect of different aspects of the handling process on the number of fibres found in a sample.

From the combination of different field sampling methods and following analytical procedures, we arrived at five different protocols by which our samples had been handled (see Table 4.2). All the stomachs collected on Polarstern expedition PS80 ( $n=49)$ were opened and rinsed out prior to the plastic investigations, but subsequent processing differed. In 19 cases (group A), the stomachs were opened and the natural diet of the polar cod was analysed (see Kohlbach et al. 2017), after which the analysed content was again preserved on formaldehyde-sea water solution.

In the other cases (group B 13 individuals and C 17 individuals), the stomach contents were removed from the stomach and preserved directly on formaldehyde-seawater solution, without any prior analysis. The remaining 23 stomachs from the other expeditions (groups D and E) were preserved intact after the dissection of the fish, and opened with scissors directly before the plastic research was conducted. The stomachs that arrived unopened were rinsed with MilliQ from the outside. During the rinsing of the stomach contents of groups $\mathrm{C}$ and $\mathrm{E}(\mathrm{n}=31$ ), a simple plastic sheet umbrella, connected to the tap, covered the sample in order to test if such addition could reduce potential secondary pollution by airborne fibres.

Scissors, tweezers, sieves and dishes were carefully rinsed with deionised water and inspected underneath the stereomicroscope before use. Precautions to prevent aerial fibre contamination were taken as far as possible by cleaning the workspace, wearing blue cotton lab coats, and as short as possible exposure of samples. Samples were covered with a clean glass lid whenever possible during processing and analysis. During microscopic analysis of samples in groups $\mathrm{D}$ and $\mathrm{E}(\mathrm{n}=23)$, a control petri dish filled with deionised water was placed next to the stereomicroscope and was checked after each sample of the previously unopened stomachs. No such controls were used in samples from groups A to C, because those stomachs had already been opened and processed to various extents before our investigations. No FTIR measurements were performed on the fibres.

Table 4.2. Overview of different method groups for the investigation of plastic ingestion by polar cod (Boreogadus saida).

\begin{tabular}{ccccccc}
$\begin{array}{c}\text { Protocol } \\
\text { Group }\end{array}$ & Expedition & $\begin{array}{c}\text { Number of } \\
\text { samples }\end{array}$ & $\begin{array}{c}\text { Stomach content } \\
\text { extracted previously }\end{array}$ & $\begin{array}{c}\text { Diet studied } \\
\text { previously }\end{array}$ & $\begin{array}{c}\text { Umbrella } \\
\text { above sieve }\end{array}$ & $\begin{array}{c}\text { Fibre } \\
\text { Control }\end{array}$ \\
\hline A & PS80 & 19 & yes & yes & no & no \\
B & PS80 & 13 & yes & no & no & no \\
C & PS80 & 17 & yes & no & yes & no \\
D & PS92 & 9 & no & no & yes & yes \\
E & HE 451.1 & 14 & no & & & yes
\end{tabular}


A one-way ANOVA followed by a Tukey's HSD post hoc test was performed to compare the number of fibres between the five different handling protocols applied. A non-parametric Wilcoxon Rank Sum test was used to evaluate the effect of an umbrella on fibre contamination during rinsing of samples between group B (no umbrella) and group C (umbrella), but showed no significant reduction of fibres in the sample. These two groups were chosen as both came from the same expedition and were further handled in the same way. The same test was used to investigate the effect of the stomach being previously opened (B and C) or not (D and E) on the amount of fibres in the sample.

For groups D and E, control petri dishes were placed next to the work space. With again a non-parametric Wilcoxon Sum Rank test, we tested whether the samples differed significantly from the controls and whether the controls differed between each other. The correlation between the number of fibres in a sample and the number of fibres in the corresponding control was tested using Pearson's correlation coefficient, which ranges between - 1 and 1 with 0 indicating no correlation. The significance of found correlations was further tested by calculating a $t$ value and corresponding $\mathrm{P}$ value based on Pearson's product moment correlation coefficient. All statistical tests were conducted using R version 3.3.1. (R Core Team 2014).

\section{Results}

The polar cod from PS80 used in this study had an average length of $78 \mathrm{~mm}$ (se 2.74), ranging from 52 to $137 \mathrm{~mm}$. Their average weight was $3.49 \mathrm{~g}$ (se 0.49), ranging from 0.83 to $18.87 \mathrm{~g}$. The fish from PS92 were larger with an average length of $107 \mathrm{~mm}$ (se 8.17), ranging from 63 to $157 \mathrm{~mm}$. Their average weight was $9.58 \mathrm{~g}$ (se 1.65), ranging from 1.71 to $24.27 \mathrm{~g}$. Fish caught during the HE451.1 expedition ranged from 44 to $62 \mathrm{~mm}$ in size, and had an average total length of $55 \mathrm{~mm}$. Their weight ranged from 0.48 to $1.56 \mathrm{~g}$, averaging at $1.01 \mathrm{~g}$.

In total, 8 particles were collected that, from their combination of size, shape and/or colour, were suspected to be plastic. Fibres were excluded from this selection and discussed separately below because of the risk of representing secondary contamination. After $\mu$ FTIR analysis, only two of these particles were confirmed to be synthetic polymers, originating from two different individuals from the expeditions HE451.1 and PS92, respectively (Figure 4.2). Of the two fish that contained plastic, the first was a 93-mm- long male caught during PS92, and the other was a fish of $46 \mathrm{~mm}$ total length caught during HE451.1. The two plastic particles were identified as two sheets as they were both soft and flexible.

According to the $\mu$ FTIR analysis, the red sheet most likely consisted of epoxy resins and had a size of $0.65 \times 0.4 \mathrm{~mm}$. The blue sheet had a kaolin base with embedded polymethylmethacrylate (PMMA) and had a size of $0.59 \times 0.17 \mathrm{~mm}$ (both spectra and details on identification can be found in Online Resource 1). Both particles were thus in the microplastic size range ( $<5 \mathrm{~mm}$; Arthur et al. 2009; Thompson 2015). Accordingly, we 

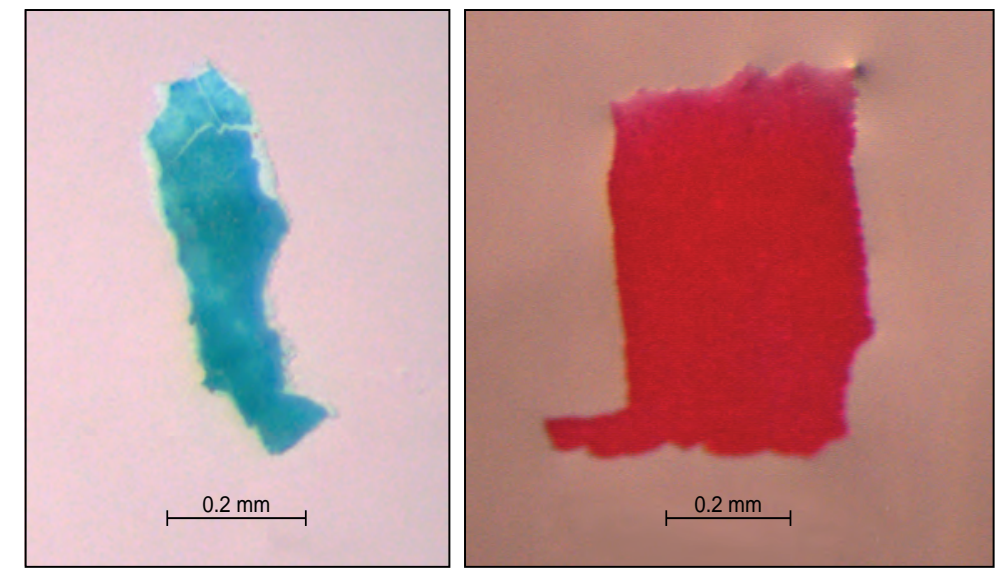

Figure 4.2. Photograph of microplastic found in stomachs of polar cod (Boreogadus saida). Left: sheet HE451.1, fish P628; Right: sheet PS92, fish P590).

found non-fibrous microplastic particles in 0 out of 51 individuals from expedition PS80, as compared to 1 of 7 individuals from PS92 and 1 of 14 individuals from the expedition to Svalbard (HE451.1). This means that the overall frequency of occurrence of non-fibrous microplastic particles in 2 out of 72 polar cods equals $2.8 \%$ for the combined expeditions. The other six particles that were suspected to be plastic initially were analysed with $\mu$ FTIR as being cotton threads $(n=3)$ and protein $(n=3)$ and therefore these particles were not counted as plastics. Protein might originate from the fish diet such as crustacean shells. The analytical spectra are presented in Online Resource 1.

\section{Fibres}

Micro-fibres were found in $90.2 \%$ of the samples, but in extremely variable quantities between and within the groups, as shown in Table 4.3 and Figure 4.3. The number of fibres per fish stomach ranged between 0 and 22, and in controls between 0 and 49. The highest mean number of fibres was found in the stomachs of the group with the longest time exposed to the air without any specific protection measures used (Group A; Figure 4.3).

Table 4.3. Average number of fibres recorded in the different sample groups of polar cod (Boreogadus saida) and in the control samples.

\begin{tabular}{cccc} 
GROUP & $\mathbf{n}$ & Average per sample $\pm \mathbf{s d}$ & Average per control \pm sd \\
\hline A & 19 & $10.9 \pm 5.3$ & \\
B & 13 & $3.1 \pm 2.8$ & \\
C & 17 & $2.3 \pm 2.1$ & $1.7 \pm 1.9$ \\
D & 9 & $5.2 \pm 7.0$ & $5.1 \pm 12.7$ \\
E & 14 & $7.3 \pm 6.7$ &
\end{tabular}




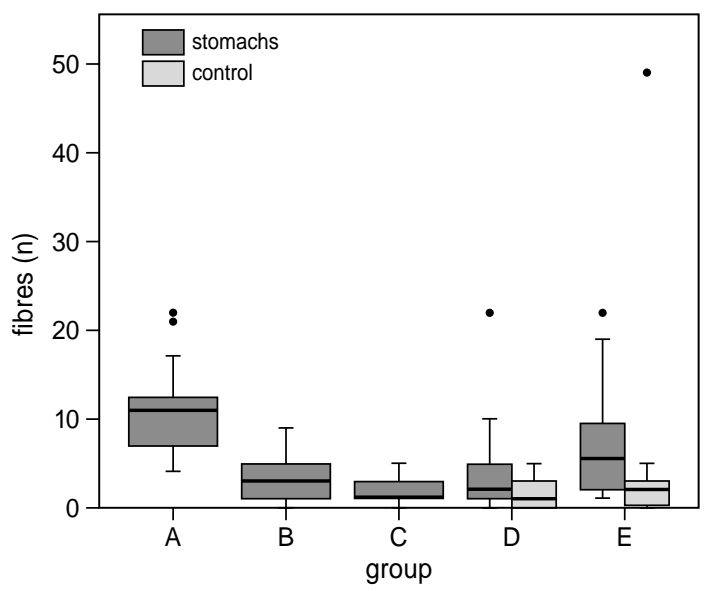

Figure 4.3. The number of fibres found in the stomachs of polar cod (Boreogadus saida; dark grey) according to the five different analysis protocols applied (group A-E; see Table 4.2 for details). Fibre controls are depicted in light grey (Group D \& E, right side). The horizontal black lines show the median number of fibres for all observations. The upper and lower limits of the coloured square boxes indicate the $25^{\text {th }}$ and $75^{\text {th }}$ percentile. The upper and lower limits of the vertical line indicate the minimum and maximum number of fibres in a group excluding the outliers (dots), which are numbers that are 1.5 times less or greater than the lower or upper percentiles, respectively.

The number of fibres in group A was significantly higher than in groups $\mathrm{B}, \mathrm{C}$ and $\mathrm{D}$ (ANOVA $\mathrm{F}_{4,67}=9.12, \mathrm{P}<0.00$; Tukey's HSD, $\mathrm{P}<0.05$ ). The number of fibres in the stomachs from group $\mathrm{E}$ was significantly higher than those in group $\mathrm{C}$ (ANOVA $\mathrm{F}_{4,67}=$ 9.12, $\mathrm{P}<0.00$; Tukey, $\mathrm{P}=0.03$ ).

There was no significant difference in the number of fibres between the remaining groups (Groups B, C and D). The stomachs that had been opened before our plastic investigations did not have significantly more fibres per sample than the ones that were first opened during our own analysis. However, as samples came from different expeditions this result should be interpreted with caution.

For both groups D and E, the mean number of fibres was higher in the analysed stomachs than in the controls. However, the difference between the treatment and its corresponding control was not significant. There was also no significant difference in the number of fibres between both controls. Although there was a match between the extremes in a sample and simultaneous control (22 fibres in the sample and 49 fibres in its control), linear regression and Pearson's correlation $(\mathrm{t}=2.028$, $\mathrm{df}=21, \mathrm{P}>0.05$ ) revealed no significant overall correlation between the number of fibres in the samples and the number of fibres in the controls that could be used to create some sort of individual correction for the number of fibres in a sample based on the number of fibres in its control. Thus, our best estimate on the impact of aerial fibre contamination during laboratory analysis are the averages found in controls of groups D and E. 


\section{Discussion}

This first study of potential microplastic ingestion by polar cod sampled over a large part of the Central Arctic Ocean (CAO) and partly dwelling in the barely accessible under-ice habitat indicates that polar cod probably do ingest microplastics, albeit at very low frequencies. However, we have no comparison for our data with other aquatic organisms living within the sea-ice habitat. Our overall result of $2.8 \%$ frequency of occurrence of ingested non-fibrous microplastic particles among 72 polar cod is similar to the level of plastic ingestion observed in the full gastrointestinal tracts of Atlantic cod (Gadus morhua) from Newfoundland, where $2.4 \%$ of 205 fish analysed contained non-fibrous plastic (Liboiron et al. 2016). Prokhorova \& Krivosheya (2013) reported two incidents in the Barents Sea of an Atlantic cod found to be entangled in fishing line and one individual with ingested plastic. By contrast, no plastic was detected in the stomachs of 114 Atlantic cod from northern Norway (Lofoten Islands and Varangerfjorden) after visual inspection of the stomach content under a microscope (Bråte et al. 2016).

Relatively high numbers of marine plastic debris have been noticed in northern fulmars from the vicinity of Svalbard. In this species, the larger picture of plastic ingestion shows decreases with higher latitude, probably related to the distance to highly urbanised areas (Kühn \& Van Franeker 2012). However, within that trend, Trevail et al. (2015a) found a slightly elevated incidence of plastic ingestion in Northern Fulmars around Svalbard and suggested a potential relation with a sixth accumulation area as modelled by van Sebille et al. (2015) and Cózar et al. (2017). Unfortunately, both the fulmar data and our polar cod data do not have the spatial resolution to evaluate further details of the Cózar et al. (2017) model.

Even though the field of microplastic research is maturing, there are still examples of recent research where fibres are considered as anthropogenic debris ingested from the environment, where no controls on airborne fibres are conducted (or reported) and potential sources of secondary pollution are neglected (e.g. Steer et al. 2017). Based on the controls conducted during our analyses, and the poorly controlled conditions during earlier steps in the collection of our samples, we have no reason to assume that synthetic fibres in our samples were derived from ingestion by the fish, as most fibres can be explained in terms of secondary contamination. If the average numbers of fibres found in the controls for groups D and E (1.7 and 5.1 fibres, respectively) are assumed to have been similar during our analyses of stomachs in groups B and C (3.1 and 2.3 fibres per stomach), the number of fibres found ingested by the fish would be negligible. The much higher number of fibres in group A, which has the same source as groups B and C, can be explained by exposure during the earlier diet study and second time of storage without special precautions against aerial fibre contamination. Accordingly, our analysis could not doubtlessly quantify ingested fibres, even if they may have well been present in the stomachs. This illustrates that it should be kept in mind that the controls for aerial contamination only cover the phase of our microscopic analysis for plastics, and that it remains unknown how much contamination occurred during the dissection of fish and stomachs 
that have been opened prior to the plastic analysis. The variation found in the numbers of fibres in the stomach contents as well as the controls suggest that there are many factors influencing the rate of pollution, including factors such as number of people present in the lab and their behaviour. As a consequence, it was not possible to assess whether polar cod were affected to a major extent by the fibres reported from sea ice (Obbard et al. 2014) and in open Arctic seawater (Lusher et al. 2015).

When studying microplastic ingestion by marine organisms, a protocol should be established that takes proper account of secondary pollution (see e.g. Torre et al. 2016; Hermsen et al. 2017; Wesch et al. 2017) in order to avoid bias in estimates of impacts of anthropogenic waste to marine organisms.

The umbrella that was tested during sample rinsing under the fume hood did not lead to a significant reduction of fibres in the samples. This suggests that rinsing was not a major contributor to airborne fibre contamination under the given circumstances. However, potential addition of fibres that might occur in tap water, as demonstrated by Kosuth et al. (2017), could not be excluded. As most of the fibres encountered in the current study were supposed to be secondary pollution, no FTIR analysis was performed. FTIR analysis would probably help to identify fibres from clothing in contrast to threadlike material derived from fishing gear due to different polymer types used. In data presentations, splitting study results in separate categories of micro- fibres, as opposed to nonfibrous plastic particles unlikely to be spread by air, will be an essential component for the future. Although at low frequency, our results do confirm that anthropogenic waste, particularly plastic debris, has not only reached pristine Arctic regions but can also be found in marine organisms closely related to the sea-ice environment. The low frequency of plastic ingestion observed in polar cod suggests that at present life dwelling under Arctic sea ice may be relatively unaffected by anthropogenic plastic pollution.

Regardless of the exact ecological consequences, plastic contamination of Arctic ecosystems and its biota likely exerts further pressure on a system that is already suffering from the impacts of global change (Wassmann et al. 2011). In the future, increased influx of Atlantic water and accelerated ice drift (Spreen et al. 2011; Walczowski et al. 2012) may enhance the advection of microplastic particles into the CAO, and hence their potential as an environmental stressor for polar cod. To better assess the regional variability of plastic ingestion by the ecological key species polar cod and potential changes over time with more certainty, we recommend geographically distributed and repeated studies. These studies should account rigorously for avoidable sources of secondary pollution, based on the experiences with this and other publications.

\section{Acknowledgements}

We thank the crews of RVs Heincke and Polarstern and Principal Scientists Antje Boetius, Ilka Peeken, Ursula Schauer and Felix Mark. Susanne Kühn is funded by the Joint Program Initiative (JPI) Oceans PLASTOX (Direct and indirect ecotoxicological impacts of microplastics on marine 
organisms) project through the Netherlands Organisation for Scientific Research (NWO) under the Project Number ALW-NWO 856.15.001. Polar research by Wageningen Marine Research is commissioned by the Netherlands Ministry of Economic Affairs under its Statutory Research Task Nature \& Environment WOT- 04-009-036. The Netherlands Polar Programme, managed by NWO, funds the $\mathrm{PhD}$ research by Fokje Schaafsma under Project No. ALW- NWO 866.13.009. Logistics for ship-based field work was provided by the Alfred Wegener Institute under research vessel cruise numbers AWI PS80, PS92 and HE451. Melanie Bergmann was funded by the Helmholtz Alliance ROBEX (Robotic Exploration of Extreme Environments), and this study contributes to the Pollution Observatory of the Helmholtz-funded programme FRAM (Frontiers in Arctic Marine Research). The study is associated with the Helmholtz Association Young Investigators Group Iceflux: Ice-ecosystem carbon flux in polar oceans (VH-NG-800) and contributes to the Helmholtz research Programme PACES II, Topic 1.5. Expedition Grant No: AWI-PS80_01; AWI-PS92_01. This publication is Eprint ID 44513 of the Alfred Wegener Institute, Helmholtz centre for polar and marine research. 
(19)

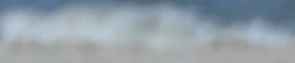

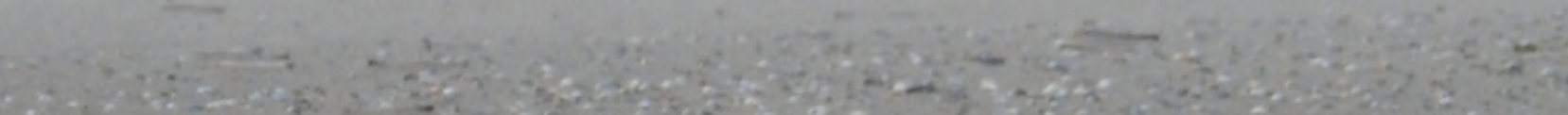

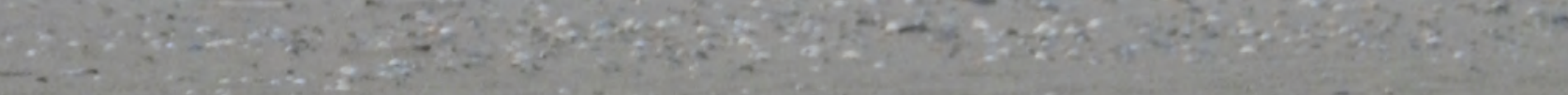

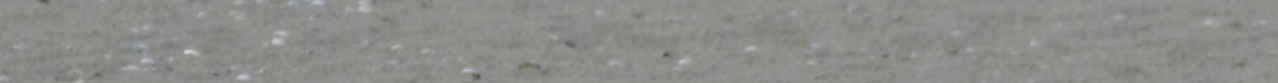

-

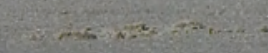

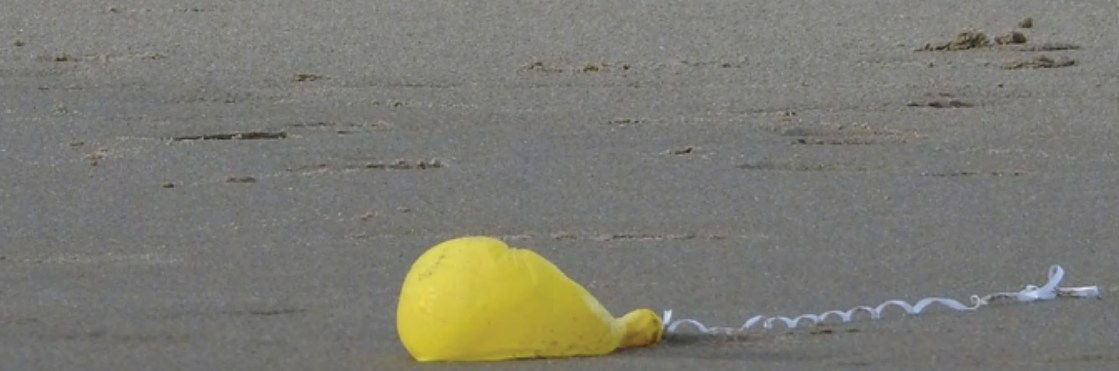




\section{The use of potassium hydroxide $(\mathrm{KOH})$ solution as a suitable approach to isolate plastics ingested by marine organisms}

Susanne Kühn, Bernike van Werven, Albert van Oyen, André Meijboom, Elisa L. Bravo Rebolledo, Jan A. van Franeker 


\begin{abstract}
In studies of plastic ingestion by marine wildlife, visual separation of plastic particles from gastrointestinal tracts or their dietary content can be challenging. Earlier studies have used solutions to dissolve organic materials leaving synthetic particles unaffected. However, insufficient tests have been conducted to ensure that different categories of consumer products partly degraded in the environment and/or in gastrointestinal tracts were not affected. In this study 63 synthetic materials and 11 other dietary items and non-plastic marine debris were tested. Irrespective of shape or preceding environmental history, most polymers resisted potassium hydroxide $(\mathrm{KOH})$ solution, with the exceptions of cellulose acetate from cigarette filters, some biodegradable plastics and a single polyethylene sheet. Exposure of hard diet components and other marine debris showed variable results. In conclusion, the results confirm that usage of $\mathrm{KOH}$ solutions can be a useful approach in general quantitative studies of plastic ingestion by marine wildlife.
\end{abstract}




\section{Introduction}

Marine pollution, and specifically plastic pollution, has gained major attention in the scientific world, as well as in the public perception. The United Nations Environment Programme (UNEP) has placed marine debris on the global agenda, and listed it as one of the major emerging environmental problems of this time (UNEP 2011). By now at least 557 species of marine wildlife have been recorded to either ingest plastic or get entangled in marine debris (Kühn et al. 2015) including several species considered threatened by international standards (Gall \& Thompson 2015). Plastic ingestion by marine wildlife such as seabirds, marine mammals and turtles has received attention in many publications since the late 1960s (e.g. Kenyon \& Kridler 1969; Baltz \& Morejohn 1976; Ryan 1987; Walker \& Coe 1990; Balazs 1985; Duguy et al. 1980). Dedicated research on ingestion of plastic by fish however, only gained significant attention from 2010 onwards (e.g. Boerger et al. 2010; Foekema et al. 2013; Lusher et al. 2013; Rummel et al. 2016; see online Supplement Table 1). The increased attention for fish can be explained by potential toxicological impacts of chemicals associated with ingested plastics and the fact that these chemicals may be consumed by humans (Rochman et al. 2012; Rochman et al. 2013). Some of these substances are additives integrated into the plastic during manufacture and processing to alter the characteristics of the product (Andrady \& Neal 2009). Other substances have been found to get adsorbed while plastic floats in seawater (Teuten et al. 2009; Mato et al. 2001). The up- take of chemical substances might have an effect not only on the organisms themselves, but also on predators higher up in the food chain, including ultimately human consumers (Tanabe et al. 2003; Tanaka et al. 2013; Van Cauwenberghe \& Janssen 2014; Rochman et al. 2015; Miranda \& de Carvalho-Souza 2016).

Seabirds, turtles and marine mammals can often only be sampled opportunistically in small numbers. However, in studies of smaller marine organisms dedicated sampling of a large number of individuals is possible (e.g. Dantas et al. 2012; Anastasopoulou et al. 2013; Foekema et al. 2013) and recommended by Galgani et al. (2013b). This large sample size has an effect on the methodological approach, as individual dissection can be difficult and time consuming in smaller organisms. In particular when the frequency of occurrence of plastic is low and for reasons of financial efficiency it can be necessary to find methods to process larger bulks of samples even though details on individual occurrence are lost. Different methods to speed up these processes have been attempted. Recently, Dehaut et al. (2016) compared different protocols using chemical solutions intended to dissolve organic matter while retaining ingested plastics. Out of six potentially suitable fluids, potassium hydroxide $(\mathrm{KOH})$ solutions proved to be the most effective for this purpose. A literature review on articles dedicated to plastic ingestion by marine organisms revealed that a $10 \% \mathrm{KOH}$ solution has been used in six earlier studies, mainly analysing the ingestion of plastic in fish (Foekema et al. 2013; Rochman et al. 2015; Lusher et al. 2016; Tanaka \& Takada 2016; online Supplement Table 1), and also during the analysis of the complete gastrointestinal tract of a beached humpback whale (Megaptera novaeangliae; Besseling et al. 2015). As KOH is mainly sold as solid material it 
was assumed that the $10 \%$ solution used in these studies ranges from 1.5-1.8 Molar depending on purity of the solid material. Dehaut et al. (2016) recommend the use of $\mathrm{KOH}$ for future research after testing pristine polymer types, whereas plastics ingested by marine wildlife is mainly composed of plastic consumer wastes that have a) undergone various treatments during production processes $\mathrm{b}$ ) have been subjected to variable periods of environmental degradation (Ter Halle et al. 2016), and c) may be affected by chemical or enzymatic processes in the gastrointestinal tract of the species studied. The $\mathrm{pH}$ in stomachs can vary depending on its fullness. It has been found that the $\mathrm{pH}$ in albatross stomachs can be as low as 1.5 (Gremillet et al. 2012) and 1.4 in fish (Lavell 1989). Enzymatic degradation (Tokiwa et al. 2009) as well as temperature might also alter the characteristics of plastic in stomachs of organisms.

Thus, in addition to fresh industrial polymer types, the scope of the current study was extended to a range of consumer products and included environmentally degraded samples collected from beaches. Furthermore the potential impacts from digestive processes were evaluated by repeating $\mathrm{KOH}$ solution tests on a selection of samples that had been exposed to stomach fluids of northern fulmars (Fulmarus glacialis), acids, or both prior to the $\mathrm{KOH}$ solution treatment. A number of so-called biodegradable or compostable plastics was included in these $\mathrm{KOH}$ solution experiments. Circumstances at sea differ tremendously from degradation processes in the compost industry (Accinelli et al. 2012; Shah et al. 2013) and increasing proportions of biodegradable plastics may persist in the marine environment for long periods of time. Such plastics may already be or become a relevant issue in studies of plastic ingestion by marine wildlife.

\section{Methods}

\section{Sample collection and preparation}

For this study 74 different types of particles were examined, 63 of which belonged to different plastic types and shapes, and an additional 11 samples of other debris or natural hard dietary items. Of the 63 plastic particle types, six belonged to biodegradable plastics and 57 to conventional plastics. 16 particles were pristine industrial polymers, 36 were taken from fresh consumer products and 14 were collected from beaches (containing samples of both environmentally degraded industrial pellets and consumer waste). An overview of particles studied is given in Table 5.1 and full details are available in the online Supplement. All items have been categorized following Van Franeker et al. (2011) and OSPAR (2015) which distinguish industrial pellets, sheets, threads, foams, fragments, threads and 'other'. For the purpose of this study the category 'other' has been split into a category for rubber-like materials and one for other plastics. Plastic debris was collected from beaches in the north of the Netherlands, especially the island of Texel $\left(53^{\circ} 1^{\prime} \mathrm{N}, 4^{\circ} 8^{\prime} \mathrm{E}\right)$ over a number of recent years. A majority of the 63 tested plastic samples $(n=48)$ had an identified polymer composition, either provided directly by the producer (supplied by Carat $\mathrm{GmbH}$ ), read from product labels, or identified using FTIR-ATR (Fourier Transform 
Infrared-Attenuated Total Reflection; Shimadzu Prestige, No. of Scans: 10, Resolution: 4 (1/cm), Spectra: 4000-600 1/cm, Apodization: Happ-Genzel, Library ATR Polymer2, IRs Polymer 2, T-Polymer). The other 15 samples are of an unknown polymer type. The $\mathrm{KOH}$ solution resistance of 11 samples of items regularly encountered in marine organisms or on beaches was tested, including seaweed, squid beaks, polychaete beaks, sheep wool, seal whiskers, fish otoliths, bird feathers, manilla rope and a metal fish hook. Other debris includes lumps of beached paraffin and palm fat. Full details can be found in the online Supplement.

\section{Sample processing}

Larger objects were cut to particles sized approximately $5 \mathrm{~mm}$ diameter as industrial plastic pellets and some natural objects had such size already. With a few exceptions (e.g. natural substances, a fish hook, a soft airgun bullet), 10 particles were combined to one sample and were then weighed to the nearest $0.0001 \mathrm{~g}$ (Sartorius 1702MP8). The 63 samples weighed on average $0.29 \mathrm{~g}$, sd $\pm 0.67 \mathrm{~g}$ (maximum weight $4.49 \mathrm{~g}$ ). They were put in glass jars and submerged in circa $20 \mathrm{ml}$ of a 1 Molar $\mathrm{KOH}$ solution (Fisher Chemicals) at $18-21^{\circ} \mathrm{C}$ (hereafter referred to as room temperature) for two days. Samples were then rinsed under tap water on a $0.3 \mathrm{~mm}$ sieve, dried at room temperature for six days and weighed again.

Table 5.1. Characterization of samples used in the KOH experiment $(n=74)$ by category, origin and (when applicable) polymer type. Classification of categories follows Van Franeker et al. (2011).

\begin{tabular}{lccc} 
Category & $\begin{array}{c}\text { Nr. of plastic samples } \\
\text { (conventional/biodegradable) }\end{array}$ & Pristine/Consumer/Beach & $\begin{array}{c}\text { Nr. of samples with } \\
\text { known polymer basis * }\end{array}$ \\
\hline Pellets & $20(18 / 2)$ & $16 / 0 / 4$ & 16 \\
Sheet & $14(11 / 3)$ & $0 / 12 / 2$ & 9 \\
Thread & $4(4 / 0)$ & $0 / 3 / 1$ & 3 \\
Foam & $7(7 / 0)$ & $0 / 4 / 3$ & 6 \\
Fragment & $8(7 / 1)$ & $0 / 8 / 0$ & 6 \\
Rubber & $8(8 / 0)$ & $0 / 6 / 2$ & 1 \\
Other synthetic & $2(2 / 0)$ & $0 / 2 / 0$ & na \\
Other debris** & 3 & $0 / 1 / 2$ & na \\
Natural*** & 8 & na & \\
\end{tabular}

*Identified polymers used in this study: $\mathrm{ABS}=$ acrylonitrile butadiene styrene, $\mathrm{CA}=$ cellulose acetate, EVA = ethylene-vinyl acetate, HD-PE = high density polyethylene, GPPS = general purpose polystyrene, LD-PE $=$ low density polyethylene, $\mathrm{NBR}=$ nitrile butadiene rubber, $\mathrm{PA}=$ polyamide, $\mathrm{PBT}=$ polybutylene terephthalate, $\mathrm{PC}=$ polycarbonate, $\mathrm{PE}=$ polyethylene, $\mathrm{PET}=$ polyethylene terephthalate $\mathrm{PP}=$ polypropylene $\mathrm{PS}=$ polystyrene, $\mathrm{PUR}=$ polyurethane, $\mathrm{PVC}=$ polyvinyl chloride, $\mathrm{SAN}=$ styrene acrylonitrile resin and different biodegradable bioplastics: Bio4Pack, Cradonyl, Mater-Bi, PHB = Polyhydroxybutyrate, PLA = polylactic acid, Wenterra

** Including: palm fat, paraffin, metal fish hook

****Including: seaweed, squid beaks, polychaete beaks, sheep wool, seal whisker, fish otoliths, bird feather, manilla rope 
Earlier publications using 10\% KOH solutions (depending on purity 1.5-1.8 M concentration) and earlier trials (see online Supplement) had shown full dissolution of fish tissue. Therefore in the current study, the lower $1 \mathrm{M}$ concentration was used in order to further reduce any potential effect on plastics. Drying the samples at room temperature was the chosen method to keep the procedure as simple as possible in other situations where no incubators are available. Furthermore this approach corresponds to the method used during the standard fulmar research (Van Franeker et al. 2011).

Tests exposing sprat (Sprattus sprattus) to a $\mathrm{KOH}$ solution of $1 \mathrm{M}$ at room temperature for two days, showed that samples of stomachs and intestines (ca. $1 \mathrm{~g}$ ), and even a whole sprat (10.5 cm, $8.9 \mathrm{~g}$ ), dissolved completely (except for the otoliths), with remains passing through a $0.3 \mathrm{~mm}$ sieve without problems. Therefore the tests with individually known plastics using $1 \mathrm{M}$ solution for two days exposure were executed.

\section{Tests in gastrointestinal fluids}

In a second experiment 22 samples were subjected to a simulated gastrointestinal environment prior to the $\mathrm{KOH}$ treatment (see Table 5.2). The treatment in $\mathrm{KOH}$ followed the same procedures as mentioned above (2 days, $1 \mathrm{M} \mathrm{KOH}$ ). After weighing, the subsamples were put in stomach fluids of northern fulmars, or in undiluted hydrochloric acid $(\mathrm{HCl}, 1 \mathrm{~N}$ standard solution, Acros Organics) or in a mixture of these for one day. Stomach fluids originated from northern fulmars hunted for human consumption on the Faroe Islands and were stored at $-20^{\circ} \mathrm{C}$ until usage. For the chemical composition of fulmar stomach fluids see: Wang et al. (2007). From literature the $\mathrm{pH}$ value in stomachs of live seabirds was known to be around 1.5 (Gremillet et al. 2012). However, when measured in the lab the $\mathrm{pH}$ of the stomach fluid was found to be around 4. To correct for this change, acid was added artificially, as stomach fluid apparently changes its $\mathrm{pH}$ value after death or during storage. To be able to differentiate between possible effects caused by either stomach fluid or acid or both samples were treated with these substances separately and in combination. After treatments, the samples were rinsed as described for the $\mathrm{KOH}$ tests above. After an initial drying period of 3 days, parts of the samples still felt wet and had excess weight. For

Table 5.2. Characteristics of samples $(n=22)$ exposed to stomach fluid and/or acids $(\mathrm{HCl})$ prior to the $\mathrm{KOH}$ experiment. None of the samples belonging to the categories "other synthetics" and "other debris" were tested. For details on polymer types and natural substances see Table 5.1.

\begin{tabular}{lccc} 
Category & $\begin{array}{c}\text { Nr. of plastic samples } \\
\text { (conventional/biodegradable) }\end{array}$ & Pristine/Consumer/Beach & $\begin{array}{c}\text { Nr. of identified } \\
\text { polymer types }\end{array}$ \\
\hline Pellets & $9(9 / 0)$ & $8 / 1 / 0$ & 8 \\
Sheet & $1(1 / 0)$ & $0 / 1 / 0$ & 0 \\
Foam & $4(4 / 0)$ & $0 / 3 / 1$ & 3 \\
Fragment & $1(0 / 1)$ & $0 / 1 / 0$ & 1 \\
Rubber & $3(3 / 0)$ & $0 / 2 / 1$ & 1 \\
Natural & 2 & na & na
\end{tabular}


practical reasons the samples then had to be stored for four weeks in plastic bags. Following storage samples were dried for an additional arbitrary period of five days before weighing for a second time.

\section{Statistical analysis}

Statistical analysis were conducted for mass differences before and after $\mathrm{KOH}$ treatments and were performed using Genstat 18th Edition (Payne et al. 2015). Potential usage of $t$-test for paired samples was evaluated by the Shapiro-Wilk test for Normality, which showed that only data for industrial pellets were sufficiently close to normality to allow usage of the $t$-test. As a non-parametric alternative, the Mann- Whitney $U$ test (equal to Wilcoxson rank-sum) was used for the tests. Significance level was taken as $\mathrm{P}<0.05$.

\section{Results}

Among the traditional plastics, most items with relatively smooth hard surfaces, (the industrial pellets and fragments) were completely unaffected with $0 \%$ average mass change, and at most 3\% change in an individual type (Table 5.3). Results for pellets by $t$-test paired samples showed differences before and after $\mathrm{KOH}$ treatment to be non-significant $(\mathrm{n}=18, \mathrm{P}=0.076)$. The Mann-Whitney $U$ tests revealed no statistical differences in any of the categories of synthetics, rubbers or biodegradables (categories and sample sizes shown in Table 5.3). The categories contain a broad range of different polymers that remain unaffected from exposure to $1 \mathrm{M} \mathrm{KOH}$ solution, and this is valid also for particles showing environmental degradation (e.g. discoloured yellowish pellets collected from the

Table 5.3. Summarized results of tests in which individually known synthetic substances were exposed to $\mathrm{KOH}$ solution of $1 \mathrm{M}$ for two days at room temperature. Tabulated are mass changes in percentages of samples after $\mathrm{KOH}$ treatment and 6 days of drying time at room temperature. None of the changes in synthetics, rubbers and biodegradable plastics were significant.

\begin{tabular}{lccccc} 
Description & $\mathbf{n}$ & $\begin{array}{c}\text { Average } \\
\text { change }\end{array}$ & $\mathbf{2}$ sd & $\begin{array}{c}\text { minimum } \\
\text { change }\end{array}$ & $\begin{array}{c}\text { maximum } \\
\text { change }\end{array}$ \\
\hline Synthetic pellets & 18 & $0 \%$ & $\pm 1 \%$ & $0 \%$ & $3 \%$ \\
Synthetic sheets & 11 & $4 \%$ & $\pm 23 \%$ & $-7 \%$ & $75 \%$ \\
Synthetic threads & 4 & $16 \%$ & $\pm 25 \%$ & $0 \%$ & $51 \%$ \\
Synthetic foams & 7 & $44 \%$ & $\pm 43 \%$ & $9 \%$ & $119 \%$ \\
Synthetic fragments & 7 & $0 \%$ & $\pm 0 \%$ & $-31 \%$ & $1 \%$ \\
Other synthetics & 2 & $-16 \%$ & $\pm 22 \%$ & $0 \%$ & $6 \%$ \\
Rubbers & 8 & $2 \%$ & $\pm 2 \%$ & $-100 \%$ & $14 \%$ \\
Biodegradable plastics & 6 & $-7 \%$ & $\pm 42 \%$ & $-100 \%$ & $38 \%$ \\
Natural Materials & 8 & $-31 \%$ & $\pm 60 \%$ & $-1 \%$ & $1 \%$ \\
Non natural materials & 3 & $0 \%$ & $\pm 1 \%$ & $-100 \%$ & $0 \%$ \\
Animal tissues & 4 & $-54 \%$ & $\pm 50 \%$ & & 0
\end{tabular}


beach) and also in tests simulating potential gastrointestinal degradation of the materials (Table 5.4). Full results are available in the Online Supplement.

Other categories of synthetic materials showed much more variable results, although mostly not in the sense of mass loss. Some types of sheet- and threadlike materials, especially foams, retained considerable moisture under the test conditions, and became heavier, apparently given insufficient time to fully dry. In a few samples, however, substantial loss of mass was observed. One sheet-like plastic of low density polyethylene (LD-PE) lost $7 \%$ of original mass. Cigarette filter material (CA cellulose acetate) lost $31 \%$ of its mass, and among the biodegradable plastic types, a sample from a bag made from PLA (polylactic acid) dissolved completely, while pellets from a material known as Cradonyl lost 33\% of their original mass. Non-plastic debris that was tested, showed resistance to $\mathrm{KOH}$ solution. In this short experiment, lack of change in a fish hook was expected, but remarkably, the fairly soft materials of beach collected lumps of paraffin and palm fat did not suffer weight loss from exposure to the $\mathrm{KOH}$ solution.

Table 5.4. Details of standard KOH solution impacts on industrial plastic pellets, pristine and from beaches, including simulated impacts from digestion processes in seabird stomachs (stomach fluids SF; acid environment $(\mathrm{HCl})$ or their combination). Shown are percentages of change in mass of plastic after treatments.

\begin{tabular}{|c|c|c|c|c|c|c|}
\hline \multirow[b]{2}{*}{ Description } & \multirow[b]{2}{*}{ Origin } & \multirow{2}{*}{$\begin{array}{l}\text { Synthetic pellets } \\
\begin{array}{l}\text { Polymer } \\
\text { type }\end{array}\end{array}$} & \multicolumn{4}{|c|}{ Treatment weight change } \\
\hline & & & $\begin{array}{c}\mathrm{KOH} \\
\text { solution }\end{array}$ & $\begin{aligned} & \mathrm{SF} \\
+ & \mathrm{KOH}\end{aligned}$ & $\begin{array}{c}\mathrm{HCl} \\
+\mathrm{KOH}\end{array}$ & $\begin{array}{c}\mathrm{SF}+\mathrm{HCl} \\
+\mathrm{KOH}\end{array}$ \\
\hline Black pellets, possibly recycled & beach & & $-0.1 \%$ & & & \\
\hline White pellets & pristine & LD-PE & $0.0 \%$ & $0.6 \%$ & $0.2 \%$ & $0.4 \%$ \\
\hline White pellets & pristine & PP & $0.0 \%$ & $0.4 \%$ & $-0.6 \%$ & $0.1 \%$ \\
\hline White pellets & pristine & PBT & $0.0 \%$ & & & \\
\hline White pellets & beach & & $0.0 \%$ & & & \\
\hline Transparent pellets & pristine & EVA VA19\% & $0.0 \%$ & & & \\
\hline Purple pellets & pristine & PET & $0.1 \%$ & $0.4 \%$ & $7.0 \%$ & $-2.9 \%$ \\
\hline Green pellets & pristine & PE-LLD recycled & $0.1 \%$ & & & \\
\hline Transparent pellets & pristine & SAN & $0.1 \%$ & & & \\
\hline White pellets & pristine & PA66 & $0.2 \%$ & $1.9 \%$ & $1.4 \%$ & $1.7 \%$ \\
\hline Transparent pellets & pristine & GPPS & $0.2 \%$ & & & \\
\hline Discoloured (yellowish) pellets & beach & & $0.2 \%$ & & & \\
\hline Black biobead (sewage usage?) & beach & & $0.2 \%$ & $0.2 \%$ & $-0.1 \%$ & $0.1 \%$ \\
\hline Transparent pellets & pristine & PC & $0.3 \%$ & $0.1 \%$ & $0.1 \%$ & $0.3 \%$ \\
\hline Transparent pellets & pristine & PA6 & $1.0 \%$ & $1.4 \%$ & $1.4 \%$ & $1.6 \%$ \\
\hline White pellets & pristine & ABS & $1.1 \%$ & & & \\
\hline White pellets & pristine & HD-PE & $1.6 \%$ & $0.5 \%$ & $0.0 \%$ & $0.6 \%$ \\
\hline Orange pellets & pristine & HD-PE & $2.8 \%$ & & & \\
\hline Total $n=18$ & & & $0.4 \%$ & $0.6 \%$ & $1.0 \%$ & $0.2 \%$ \\
\hline
\end{tabular}


Among non-plastic materials found in, for example, bird stomachs, many substances were dissolved by the KOH solution (Polychaete jaws, sheep wool, seal whiskers, bird feathers) but others were largely retained, two notable examples being fish otoliths and squid beaks. The difference between squid beaks and polychaete worm jaws is remarkable as both are assumed to be built from the same natural polymer, keratin. Table 5.3 summarizes the results by category of material. Full details are given in the Online Supplement.

Balloon rubber, likely largely consisting of natural latex, did not show a decrease in weight, but rather, exceeded its original mass after processing. It was also seen that the exposure of balloon rubber to the stomach fluid of northern fulmars triggers a change in material characteristics. The rubbery structure was lost and the material became fluid and sticky. These results correspond to the personal observations of the authors when finding balloon remains or similar substances in the stomachs of northern fulmars: although not evident inside the stomach, once removed and laid out to dry, many of these items become a mucoid mud rather than a dried particle.

Foamed materials in particular when exposed to fulmar stomach fluids, sometimes showed an excessive increase in weight (see Online Supplement) indicating that the oily substances in these stomach fluids had settled in the airy spaces and were not removed by simple rinsing in cold water and subsequent drying. Results for industrial pellets (Table 5.4) indicate, however, that the conclusion is justified that exposure to stomach fluids or $\mathrm{HCl}$ does not make plastic polymers more prone to dissolving in $\mathrm{KOH}$ solution.

\section{Discussion}

When promoting a new method it is important to make sure that it works under various circumstances. Exposing stomachs and their contents to $\mathrm{KOH}$ solutions is a newly proposed method to efficiently study plastic ingestion in situations where individual visual separation of plastics from gastrointestinal tracts is too difficult or too labour intensive. However, the assumption that indeed organic components are dissolved and all synthetic materials are retained needs proper testing. Such testing should include not only virgin polymer types, but must consider a wide variety of synthetic consumer products, also taking into account the potential impacts from material degradation in the environment or in the gastrointestinal tracts of species studied.

During this study, plastics of different categories, polymer types and origins as well as natural substances and items were tested and exposed to a $\mathrm{KOH}$ solution in a concentration which easily dissolved the tissues of a small fish species (Sprattus sprattus, solution of 1 Molar $\mathrm{KOH}$ concentration, applied during 2 days at room temperature). The results show that a wide range of polymer types, in different shapes and forms, and partly subjected to earlier environmental degradation were not affected by this $\mathrm{KOH}$ solution treatment. As was reported by Dehaut et al. (2016) one of the few synthetic substances that was dis- solved in $\mathrm{KOH}$ solution was cellulose acetate (CA), in this study represented by a cigarette filter. Cigarette filters are often found in high numbers on tourist beaches 
(Ocean Conservancy 2016). Besides additives added to the polymer during production processes, the toxic compounds of cigarettes are attached to the filters and have been proved to be toxic to fish (Slaughter et al. 2011). However, in plastic samples from the marine environment, cigarette filters seem infrequent. Once soaked, the material will probably disintegrate and loose fibrous elements (Puls et al. 2011) and will not be easily detected in the water or in animal stomachs or intestines. In long term studies of plastic ingestion in northern fulmars in the North Sea (Van Franeker et al. 2011) there are only a few records of ingested cigarette filters. Results for the Netherlands suggest maybe 3 such cases in over a 1000 stomachs analysed.

Remarkably, among several samples representing polyethylene (PE) one bag labelled as being made of LD-PE showed some degradation, with 7\% mass loss in the experiment. The reason for this contradictory case is not clear, but could indicate degradation or production issues.

Two out of six biodegradable plastics showed substantial degradation following $\mathrm{KOH}$ treatment: fragments from a bag made from polylactic acid (PLA) disappeared completely, and granules of a material called Cradonyl lost one third of their original mass. In 2014, bio-based and biodegradable plastics together represented $1 \%$ of the global plastic production (EUBP 2016). Even though the degradation of bioplastics in the ocean is very slow (Accinelli et al. 2012; O'Brine \& Thompson 2010), this suggest that only a small share of the marine debris currently consists of this material. In the near future, the proportion of such polymers will likely remain low, even though the production is expected to quadruple by 2019 (EUBP 2016). However, the effects of this development should be monitored, as on the longer term this might have consequences on the composition of plastics found in the ocean and subsequently in marine organisms.

Treatment of samples with seabird stomach fluids and acids to simulate digestive processes did not change the results of the $\mathrm{KOH}$ tests. The results of this study indicate that the chosen $\mathrm{KOH}$ solution concentration ( $1 \mathrm{M}$, two days at room temperature) can be safely applied in a quantitative study to estimate levels of plastic ingestion by small fish. Proportions of ingested plastics that are lost through $\mathrm{KOH}$ treatment will be relatively small. The tests, however, also showed that drying times needed to get rid of moisture in foamed plastics were substantial, and that such problems were aggravated by fatty stomach fluids. This implies that when a study species ingests a substantial proportion of foamed materials, additional procedures for cleaning and drying the isolated plastics should be considered and tested. Standard protocols often advise to quantify plastic ingestion by marine wildlife in terms of plastic mass (Ryan et al. 2009; Van Franeker et al. 2011; OSPAR 2015; Provencher et al. 2017). Components of the dissolved KOH could also contribute to the increased weight.

An initial trial with samples containing a mix of plastics collected on beaches (see Online Supplement) suggests that the conclusions are similarly valid with increased concentrations of $10 \% \mathrm{KOH}$ solutions. Results by Dehaut et al. (2016) suggest that such a concentration can also be applied safely at a temperature of $50^{\circ} \mathrm{C}$. On the other hand, a preliminary test on digestion of seabird intestines under our standard exposure of $1 \mathrm{M}$ 
$\mathrm{KOH}$ for 2 days, showed them to be immune to that concentration even if duration was prolonged. Depending on the tissue to be dissolved, concentration and duration may need to be increased to achieve disintegration of the organic material. The fulmar intestines tested in these studies were found to dissolve completely in three days in $5 \mathrm{M} \mathrm{KOH}$ solution at $60^{\circ} \mathrm{C}$. Resistance of plastics to such other conditions will vary and needs additional testing. When studies of plastic ingestion are combined with research of the diet, care should be taken with $\mathrm{KOH}$ applications. Important diet items such as fish otoliths and squid beaks appear to be resistant, but some other similar components disappeared. Therefore it is recommended to test typical prey items occurring in the species studied to make sure they are resistant to the $\mathrm{KOH}$ dissolution procedure.

Overall, the results of this study confirm in detail that the $\mathrm{KOH}$ approach to separate plastics from organic matter is a valuable and practical method in studies of plastic ingestion by marine wildlife.

\section{Acknowledgements}

The first author was funded by the Joint Program Initiative (JPI) Oceans PLASTOX (Direct and indirect ecotoxicological impacts of microplastics on marine organisms) project through the Dutch Science Foundation (NWO) under the project number ALW-NWO 856.15.001. Christopher Miele checked grammar and spelling of this manuscript. Marine litter studies by Wageningen Marine research receive long- term support from the Netherlands Ministry of Infrastructure and the Environment. We want to thank the anonymous reviewer for the critical revision of the earlier version of this manuscript. 


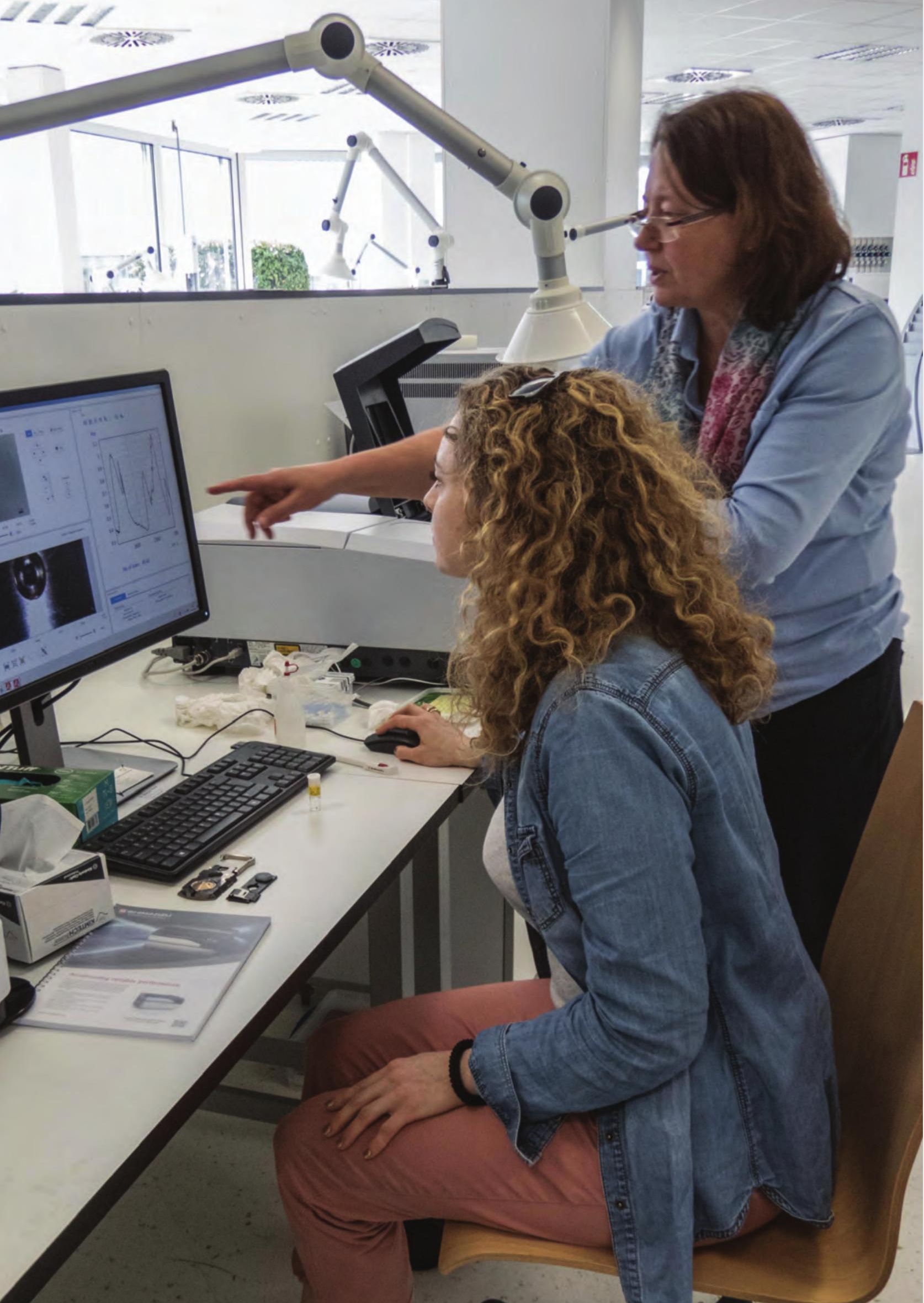




\section{Details of plastic ingestion and fibre contamination in North Sea fishes}

Susanne Kühn, Jan A. van Franeker, Anastasia M. O'Donoghue, Ailynn Swiers, Marrit Starkenburg, Bernike van Werven, Edwin Foekema, Enya Hermsen, Marion Egelkraut-Holtus, Han Lindeboom 


\begin{abstract}
This study combines published datasets with unpublished data on plastic ingestion in several North Sea fish species. The combined dataset of 4389 individuals from 15 species allows the analysis of spatial distribution and temporal variability of plastic uptake in fish. Airborne fibre contamination was observed to be the main contributor to fibres encountered in the samples. The number of fibres in samples was strongly related to the time needed to process a sample, not to the number of individual fishes in the sample. Accurate correction for secondary fibre contamination was not possible, but corrections required would be similar to fibre numbers observed in the samples. Consequently, all fibres were omitted from further analysis. The frequency of occurrence and the average number of plastics in fish is generally low $(1.8 \%$ and 0.022 pieces per organism respectively), with only cod having a higher prevalence (12.3\%). While latitude of catch locations influences plastic uptake in fish, no correlation with the distance to the coast was found. Slightly less plastics were ingested in winter, and a decrease in plastics ingested was observed between 2009 and 2018. These factors should be considered when fish species, catch location and time are discussed as indicators for plastic pollution in the European Marine Strategy Framework Directive. We recommend considering demersal cod and pelagic sprat as two species suitable for monitoring plastic ingestion in biota, both on the seafloor and in the water column.
\end{abstract}




\section{Introduction}

Plastic ingestion by fish has gained increased awareness in recent years, resulting in a growing body of literature (Markic et al. 2019). Anthropogenic debris, such as cans, ropes and horse shoes were found in tiger sharks (Galeocerdo cuvier) as early as 1912 (Gudger 1949). The first record of plastic in fish originates from 1964, when Kubota (1990) found $62.5 \%$ of longnose lancetfish (Alepisurus ferox) contained plastics, including plastic soft drink bottles. In 2015, at least 92 fish species were recorded with ingested plastics (Kühn et al. 2015). More recently, this was shown to be the case for 232 fish species (Markic et al. 2019). This indicates an increase in the general interest in the topic, which is reflected in high scientific output.

As microplastics ( $<5 \mathrm{~mm}$; Arthur et al. 2009) occur throughout the water column and on the seafloor, they are available to a wide range of marine fish species (Lusher 2015). Once ingested, plastics can cause a reduction in reproduction capabilities, as well as in growth and survival rates (reviewed by Foley et al. 2018), but the pathways are not well understood. For pollutants adsorbed from surrounding seawater, models suggest that the additional role of plastics may not be important as these pollutants are also present in the natural food of the organisms (Gouin et al. 2011; Koelmans et al. 2013). However, in nature, the actual impact is unclear. For chemicals, for which plastic forms the main source such as built-in additives, this may be different (SAPEA 2019). These chemicals may accumulate in fish tissue and consequently in the consumers of these fish, which include not only marine predators but also humans (Rochman 2015). Trophic transfer as one of the pathways of plastics in predators is difficult to observe in the wild. However, one particular example was witnessed in Canada, where little auks (Alle alle) with plastics in their stomachs were eaten by goosefish (Lophius americanus). Furthermore, fish caught by rhinoceros auklets (Cerorhinca monocerata) contained microplastics, indicating trophic transfer (Hipfner et al. 2018), but trophic transfer has also been observed between different fish species (Welden et al. 2018).

Plastic ingestion by fish in the North Sea has been reported. One early report originates from 1972 where Kartar et al. (1976) found that 5 to $12 \%$ of four fish species contained plastics. Years later in 2013, two sets of new data on plastic in fish in the North Sea area were published. Foekema et al. (2013) only found plastic in $0-13 \%$ of individuals from seven fish species. Lusher et al. (2013) reported much higher frequencies of occurrence with 23.5 to $59 \%$. However, these studies are hard to compare, as Foekema et al. (2013) excluded light-weight fibres while Lusher et al. (2013) included them. Since then, several further publications reflect a major interest of scientists, policy and the public in plastic pollution in fish (Rummel et al. 2016; Collard et al. 2017; Hermsen et al. 2017; Murphy et al. 2017; Steer et al. 2017; McGoran et al. 2018).

While northern fulmars (Fulmarus glacialis) are used by OSPAR and the EU (OSPAR 2017) as a monitoring species for plastic litter in the marine environment, additional data on plastic ingestion by fishes can provide valuable complementary data as they cover other foraging habitats than the surface-feeding fulmars (Galgani et al. 2013b). The expected 
lower abundances of plastic in fish indicates that large sample sizes are needed to gather reliable data. For this study, new data on almost 2800 fishes from 13 species were collected, which were subsequently combined with the original data from two earlier studies (Foekema et al. 2013; Hermsen et al. 2017). These combined efforts resulted in plastic ingestion data for 4389 fish from 15 species covering different years and seasons, habitats and locations throughout the North Sea. Several of these species serve as staple food for marine birds and mammals, and many species have a major commercial fisheries interest. A sample of 120 Norway lobsters (Nephrops norvegicus) was included as they were available as a bycatch in our trawl samples. The species has commercial relevance, and has been reported to ingest plastics on a regular basis (Murray \& Cowie 2011; Welden \& Cowie 2016). A large and diverse dataset is essential to adequately advise policymakers on potentially suitable monitoring species for the European Marine Strategy Framework Directive (MSFD).

\section{Material and Methods}

This study combines three datasets. Data by Foekema et al. (2013) includes 1203 fishes from seven species, caught during Dutch International Bottom Trawl Surveys (IBTS) in 2010 and 2011. Hermsen et al. (2017) analysed the stomach contents of 400 fishes from 4 species from 2013, also caught during IBTS.

The new dataset consists of 2786 fish, collected opportunistically for plastic studies between 2009 and 2018 during fish surveys or fishing gear trials in different North Sea regions. For convenience, hereafter the 120 aforementioned Norway lobsters are included when the term 'fish' is used. During Dutch IBTS in January and February of 2013 and 2018, 1172 fishes were caught. In January 2013, an additional 958 sprat were collected during a grebe survey conducted close to the Dutch shore. When many foraging birds were observed, fish was sampled with a beam trawl (Leopold et al. 2013). International Herring Acoustic Surveys (HERAS) collect data on distribution and population size of clupeid fish each summer, with samples (117 herring) used in this study collected in July 2016. Between July 2007 and August 2010 the newly developed Surface and Under Ice Trawl (SUIT; Van Franeker et al. 2009; Flores et al. 2012) was tested at the Frisian Front (70 km north of the Dutch coast), and at the Marsdiep area (between the Dutch mainland and the island of Texel). During this trial, 539 fishes of different species were obtained.

For the new dataset, 13 species were studied, among which two species were only available in very small numbers (anchovy $n=4$, three-spined stickleback $n=1$, hereafter called stickleback). Clearly results for these small samples have no species specific information value. They are nevertheless included because we advocate the broadest possible species composition and their inclusion means no harm to our overall data analyses. In the North Sea five species of sandeel (Ammodytidae) occur. Identification to species level is difficult and therefore is often refrained from during surveys (Camphuysen et al. 2017). For this study Ammodytidae are pooled as one group. 
The combination of studies results in a total of 4389 fish stomachs (Table 6.1) used for the analyses in this paper. Full details on the different species sampled, gear type used, year of catch, season, approximate latitude and longitude can be found in the Online Supplement Table 6.1.

Fish stomachs from the Foekema et al. (2013) study were exposed individually to $10 \%$ potassium hydroxide $(\mathrm{KOH})$ to dissolve the organic matter and facilitate the recognition of potential plastics. $\mathrm{KOH}$ has been proven to be an efficient solvent, as organic matter is dissolved within days, while plastics remain intact (Dehaut et al. 2016; Kühn et al. 2017). Any remaining items were sieved $(0.2 \mathrm{~mm})$ and analysed under a stereomicroscope. Hermsen et al. (2017) followed a similar approach of using $10 \% \mathrm{KOH}$ to dissolve organic material. While 100 individuals were analysed separately, the remaining 300 were combined in batches of several individuals from the same species, catch locations and date. Samples were then sieved on a $20 \mu \mathrm{m}$ sieve and checked for plastics under stereomicroscope. Both studies recorded the longest diameter of items. Since 12 items from Foekema et al. (2013) were recorded within a range of 2-3 mm, these were averaged at $2.5 \mathrm{~mm}$. Additionally, since the Foekema et al. (2013) dataset did not measure items $<0.2$ $\mathrm{mm}$, average size might be slightly overestimated. In this paper we distinguish between 'plastic particles' and 'fibres'. Particles include industrial granules and microbeads, sheets, threads, foams, fragments and recognisable other objects. Note that threadlike materials in

Table 6.1. Overview of sampled species, sample numbers ( $n$ ), catch period, average size (fish: total length, lobster: total length, tip rostrum to tip tail, $\mathrm{cm}$ ) and mass (wet weight, g). All species were allocated a demersal (D) or pelagic (P) feeding habitat.

\begin{tabular}{|c|c|c|c|c|c|c|}
\hline \multirow[t]{2}{*}{ Species } & \multirow[t]{2}{*}{ Habitat } & \multirow[t]{2}{*}{$\mathrm{n}$} & \multicolumn{2}{|c|}{ Period } & \multirow{2}{*}{$\begin{array}{c}\text { Size } \\
\text { avg cm }\end{array}$} & \multirow{2}{*}{$\begin{array}{l}\text { Mass } \\
\operatorname{avg} g\end{array}$} \\
\hline & & & from & to & & \\
\hline Sprat Sprattus sprattus & $\mathrm{P}$ & 1516 & 2010 & 2016 & 10.3 & 7.3 \\
\hline Herring Clupea harengus & $\mathrm{P}$ & 1143 & 2010 & 2018 & 20.9 & 128.9 \\
\hline Sandeel Ammodytes spp & $\mathrm{P}$ & 295 & 2009 & 2010 & 5.6 & 0.2 \\
\hline Plaice Pleuronectes platessa & $\mathrm{D}$ & 250 & 2018 & & 26.2 & 179.7 \\
\hline Haddock Melanogrammus aeglefinus & $\mathrm{D}$ & 218 & 2011 & 2018 & 27.8 & 198.6 \\
\hline Whiting Merlangius merlangus & $\mathrm{D}$ & 187 & 2010 & 2018 & 26.6 & 231.2 \\
\hline Grey gurnard Eutrigla gurnardus & $\mathrm{D}$ & 171 & 2010 & & 19.5 & 78.6 \\
\hline Mackerel Scomber scombrus & $\mathrm{P}$ & 131 & 2010 & 2018 & 25.8 & 183.1 \\
\hline Norway lobster Nephrops norvegicus & $\mathrm{D}$ & 120 & 2018 & & 12.9 & 28.7 \\
\hline Cod Gadus morhua & $\mathrm{D}$ & 114 & 2011 & 2018 & 55.7 & 2928.7 \\
\hline Horse mackerel Trachurus trachurus & $\mathrm{P}$ & 101 & 2010 & & 20.7 & 78.2 \\
\hline Dab Limanda limanda & $\mathrm{D}$ & 100 & 2013 & & 18.2 & \\
\hline Norway pout Trisopterus esmarkii & $\mathrm{P}$ & 38 & 2018 & & 15.2 & 26.5 \\
\hline Anchovy Engraulis encrasicolus & $\mathrm{P}$ & 4 & 2010 & & & \\
\hline Stickleback Gasterosteus aculeatus & $\mathrm{P}$ & 1 & 2013 & & 5.9 & 1.5 \\
\hline All species & & 4389 & 2009 & 2018 & 19.3 & 194.4 \\
\hline
\end{tabular}


this categorisation are different from fibres. Threads originate from e.g. multifilament ropes and nets or monofilament lines or nets, which are much thicker than the dust-fibres originating from e.g. synthetic or natural clothing. We follow Tanaka \& Takada (2016) in using a diameter of $50 \mu \mathrm{m}$ to distinguish between the thinner fibres and the thicker threadlike particles.

For the new data, a series of at least 20 fishes per sample group (fish from the same species, catch date \& location) were studied individually. In a series of 200 sprat (from the beam trawl in 2013), stomachs were opened and their content analysed directly under the microscope. All other samples were treated with $\mathrm{KOH}$ in glass jars with the volume of $\mathrm{KOH}$ at least once that of the sample. Generally, the exposure of $1 \mathrm{M} \mathrm{KOH}$ (FisherChemicals) at room temperature for 3-4 days was sufficient to dissolve the organic matter. Only when a large amount of natural prey was present, a stronger concentration of $5 \mathrm{M}$ was used or the exposure time was extended. Tests by Lenz et al. (2016) show, that plastics can withstand much higher concentrations $(20 \mathrm{M})$ without property changes. As the presence of plastics was rare, and individuals showed rarely more than one plastic item per fish, it was decided to combine stomach contents of fish in batches to speed up the process. Initially small batch size was used, e.g. 5 fish, but depending on results and numbers to be analysed, batch size was increased to a maximum number of 51 fish. In batches, the frequency of occurrence (\%FO) was calculated on the assumption that no fish had more than one plastic particle in its stomach.

After rinsing the samples in a $30 \mu \mathrm{m}$ mesh-sized sieve with fresh tap water to remove the $\mathrm{KOH}$ and digested organic material, the samples were poured in a Bogorov counting chamber and analysed under a microscope (Zeiss Discovery V8 stereomicroscope with maximum 50× magnification, AxioCam MRc with AxioVision 40 V4.8.2.0 software, Göttingen, Germany). Photos of suspect particles were taken with either a Leica EZ4 W microscope or the Zeiss microscope and the longest diameter of the items was measured.

To identify polymer composition of ingested particles by FTIR, Foekema et al. (2013) selected six plastic items with visual characteristics representative of all the types of particles found. High costs prevented analyses of all particles. Unfortunately the original particles appear to be lost, preventing analysis at the current much cheaper rates. To identify the polymer type of two plastics found in Hermsen et al. (2017), the authors used FTIR analysis as well. For the new data, FTIR was applied to a subsample of 69 particles, which contained a large proportion of items visually judged to likely be natural materials, such as broken fragments of e.g. fish scales or fish bones. This approach was chosen to check whether our visual surveys underestimated presence of plastics.

Particles larger than $1 \mathrm{~mm}$ were analysed with FTIR (IRSpirit Shimadzu, wavelength $400-4000 \mathrm{~nm}, 45$ scans, Shimadzu Germany). Smaller particles underwent $\mu$ FTIR analysis (IRTracer-100, FTIR Microscope AIM-9000, Shimadzu Germany) using a DC-3 diamond compression cell and measured in transmission mode at different places on the sample to reduce the impact of potential surface fouling. Spectra were compared to more than 14500 spectra from standard libraries (Shimadzu Libraries, STJapan-Europe, standard database from Biorad Sadtler and other libraries) and were accepted when the 
overlap was scored $>75 \%$. Spectra between 75 and $80 \%$ match score were accepted when additionally judged as being plastics by Shimadzu FTIR experts.

\section{Airborne fibre contamination}

In our first partial dataset (Foekema et al. 2013), fibres were found to be frequently present. However, in later tests where part of the procedure was conducted under clean-air conditions, fibres were rare. Lacking options for further improvement and controls, it was decided to further ignore fibres and omit them completely from presentation of results. In the second dataset (Hermsen et al. 2017), it was possible to conduct the full sequence of gut sampling and content analysis under strict clean-air conditions.

For the new dataset no laminar flow cabinet was available. Nevertheless, mitigation measures for this study were taken whenever possible. Cotton lab coats were worn, lab equipment was thoroughly cleaned before use and samples were kept covered when possible. Details on fibres in our individual samples, batch samples and controls were used to gain insights into what influences fibre contamination and the effect on fibre numbers in the sample.

In the new study, detailed fibre counts in samples and controls were performed for a series of 1002 fishes, which were either individually or batch-processed (details in Online Supplement Table 6.2). In the same dataset (IBTS 2018), a number of cod were assessed in the same way, but in the fibre analyses they were not used as they were done individually only. For controls, a clean Petri dish was filled with MilliQ, placed next to the sample and followed the full process from when the stomach was opened onwards. Whenever the stomach sample was exposed to air, the lid of the control was opened simultaneously. After the fish sample was analysed (for plastics and fibres), the control petri dish was checked and the number of fibres was recorded.

The time to process an individual sample or a batch containing multiple fish is assumed to be comparable. From this sample there were 212 individually analysed fish and 790 fish analysed in 73 batches.

\section{Spatial distribution}

All species were allocated to a feeding habitat, either demersal or pelagic, according to Heesen et al. (2015) and Camphuysen et al. (2017) (Table 6.1). A total of 1160 fish from 7 species (including Norway lobster) usually feed close to the bottom (demersal), while 3229 fish representing 8 species belong to pelagic species. Both groups were compared in terms of $\% \mathrm{FO}$ and the average number of items per fish.

All individuals were grouped in catch locations, once north and south of $53^{\circ} \mathrm{N}$, where the southern part of the North Sea starts narrowing down in the direction of the English Channel area and once north and south of $55^{\circ} \mathrm{N}$ to allow comparison with data by Foekema et al. (2013). Additionally, the relationships of latitude and plastic uptake in individual fishes were tested.

Earlier studies have indicated a relationship between plastic uptake and the distance to the coast, with fish having more plastics close to the coast (Steer et al. 2017; Pozo et al. 
2019). With the function 'Near of the application' by ArcGIS (Version 10.6.1), the distance to the nearest coastline was calculated. Linear regression was used to identify differences in the distance to the coast and the $\% \mathrm{FO}$ of plastic in fish.

\section{Temporal variation}

Fishes collected for this study originate from 6 distinct years, covering a 10-year period from 2009 to 2018 (Table 6.1). Variation within a year covered different months of the year. Summer has been defined as the months July and August, autumn consists of the months September and October while winter includes the months January and February. In the remaining months, no fish was caught. As the biggest gap in data occurred in spring, the sequence of month and seasons was defined as summer - autumn - winter. The \%FO and the average number of plastics ingested were compared for all species combined, demersal and pelagic species respectively and for sprat, herring and cod separately to better understand how these species may influence the results.

\section{Data analysis}

As data of this study include large proportions of zero values, data distributions are extremely skewed, which complicates statistical interpretation of results. Average number of items cover the number of plastic particles (or fibres) divided by all organisms investigated (population average).

Frequencies of occurrence were defined as the proportion of affected organisms in relation to the total sample size. The smaller the sample size, the less reliable are these observed \%FO's. According Confidence Intervals (CI) were calculated using the $95 \%$ confidence limits as proposed by Provencher et al. (2017) (Jeffreys method; http://epitools. ausvet.com.au/content.php?page=CIProportion).

Genstat's Restricted Maximum Likelihood (REML) analysis was used for an integrated analysis of the different factors potentially affecting the uptake of plastics and their interactions (Genstat 19 ${ }^{\text {th }}$ Edition). Factors included species, latitude, distance to coast, year and season. Habitat was not included, because per species one habitat was assigned and Genstat did not allow their combined use in the REML. Although REML in principle assumes a normal distribution of the response variate (the number of plastic particles in an individual), it is not a critical assumption (O'Neill 2010). With the results of the REML analyses in mind, potential factors influencing the data were further considered individually in more detail using tests for binomial proportions and non-parametric tests.

Where data could be grouped in two sets (e.g. pelagic versus demersal species) differences in \%FO's were tested using either the 2 sample-test (http://epitools.ausvet.com.au/ content.php?page=z-test-2) or a General Linear Model (GLM) over binominal proportion (Genstat 19th Edition).

For sequential multi-value variables, such as latitude, distance to coast or year, different approaches were used. GLM analyses of binomial proportions were applied to grouped data (e.g. by degree latitude, season, month or year of collection). Individual data of plastic particle numbers were viewed in simple linear regressions as an indication of 
potential correlations with variables (but without statistical relevance). Where relevant, average numbers of plastic in fish were evaluated using the non-parametric MannWhitney-U test (Genstat $19^{\text {th }}$ edition), where no normal distribution of the data is required. Significance level for all tests was set at $\mathrm{P} \leq 0.05$.

\section{Results}

\section{Abundance of plastic particles and fibres}

Data for plastic particle and fibre abundance in 4389 individuals from 15 North Sea fish species are shown in Table 6.2 and further specified in later paragraphs. In batch samples, the $\%$ FO was estimated as being one plastic item per individual, as in samples where fish were analysed both individually and in batches, only three individual fishes (two sprat and one mackerel) contained more than one, actually two plastic pieces. In batches, the number of fibres occasionally exceeded the number of fishes in the batch analysed, in which case $\%$ FO was assumed to be $100 \%$, and the numerical abundance taken as average per individual fish in the batch.

Table 6.2. Result overview shows plastic particles, uncorrected fibres and fibres found in the control per species. Details are given for the sample size (n), the average number of items (avg) including the standard error $( \pm$ se) and the frequency of occurrence (\%FO). Fibre calculations and controls were not available for all individuals, therefore sample numbers differ for plastic particle abundance and fibre abundance.

\begin{tabular}{|c|c|c|c|c|c|c|c|c|c|c|c|c|}
\hline \multirow[b]{2}{*}{ Species } & \multicolumn{4}{|c|}{ Plastic particles } & \multicolumn{4}{|c|}{ Fibres (uncorrected) } & \multicolumn{4}{|c|}{ Fibre controls } \\
\hline & n fish & avg & $\pm \mathrm{se}$ & $\%$ FO & $\mathrm{n}$ fish & avg & \pm se & $\% \mathrm{FO}$ & $\mathrm{n}$ fish & avg & \pm se & $\% \mathrm{FO}$ \\
\hline Sprat & 1516 & 0.013 & 0.002 & 1.2 & 141 & 0.000 & 0.000 & 0.0 & 141 & 0.000 & 0.000 & 0.0 \\
\hline Herring & 1143 & 0.019 & 0.006 & 1.2 & 511 & 0.497 & 0.109 & 14.5 & 511 & 0.200 & 0.061 & 6.8 \\
\hline Sandeel & 295 & 0.017 & 0.005 & 1.7 & 0 & & & & 0 & & & \\
\hline Plaice & 250 & 0.020 & 0.008 & 2.0 & 243 & 0.840 & 0.131 & 32.1 & 243 & 0.305 & 0.066 & 14.8 \\
\hline Haddock & 218 & 0.032 & 0.012 & 3.2 & 121 & 0.835 & 0.183 & 32.2 & 121 & 0.421 & 0.132 & 15.7 \\
\hline Whiting & 187 & 0.053 & 0.025 & 3.7 & 81 & 0.963 & 0.203 & 43.2 & 81 & 0.395 & 0.126 & 17.3 \\
\hline Grey gurnard & 171 & 0.000 & 0.000 & 0.0 & 0 & & & & 0 & & & \\
\hline Mackerel & 131 & 0.023 & 0.017 & 1.5 & 47 & 2.234 & 0.911 & 53.2 & 47 & 1.362 & 0.884 & 19.1 \\
\hline Norway lobster & 120 & 0.025 & 0.012 & 2.5 & 120 & 0.267 & 0.065 & 16.7 & 120 & 0.117 & 0.043 & 7.5 \\
\hline Cod & 114 & 0.132 & 0.034 & 12.3 & 0 & & & & 0 & & & \\
\hline Horse mackerel & 101 & 0.050 & 0.026 & 4.0 & 0 & & & & 0 & & & \\
\hline Dab & 100 & 0.000 & 0.000 & 0.0 & 100 & 0.000 & 0.000 & 0.0 & 100 & 0.000 & 0.000 & 0.0 \\
\hline Norway pout & 38 & 0.026 & 0.026 & 2.6 & 38 & 1.684 & 0.342 & 63.2 & 38 & 1.079 & 0.300 & 39.5 \\
\hline Anchovy & 4 & 0.000 & 0.000 & 0.0 & 0 & & & & 0 & & & \\
\hline Stickleback & 1 & 0.000 & 0.000 & 0.0 & 0 & & & & 0 & & & \\
\hline All species & 4389 & 0.022 & 0.003 & 1.8 & 1402 & 0.598 & & 21.0 & 1402 & 0.270 & 0.042 & 9.8 \\
\hline
\end{tabular}


On average, $1.8 \%$ of fish investigated had plastic particles in the digestive tract, with an average and standard error of $0.022 \pm 0.003$ particles per individual. Cod is the only species with a relatively high $\%$ FO of plastic particles at $12.3 \%$. All other species remain well under 5\%. Grey gurnard and dab ( $\mathrm{n}=171$ and 100 respectively) did not contain any plastics. The same applies to anchovy and stickleback, however sample sizes for these species were extremely low with four and one individuals respectively. More details on the results on plastic particle abundance from the three separate datasets can be found in the Online Supplement Table 6.2.

Hermsen et al. (2017) conducted their research in a laminar flow cabinet and zero fibres were found in both fish samples and in controls. For the newly added data, the work process was made as clean as possible, but clean air procedures were not possible. In order to evaluate true and secondary fibre abundance under these work conditions, fibres in samples and controls were quantified in high detail in a subsample of 1002 fish. Among these, 212 fish were investigated individually, while 790 fish were processed in 73 batches. Table 6.2 provides fibre data in samples and controls for the combination of the 400 fishes in the Hermsen et al. (2017) study and the 1002 fishes added here.

The average number of fibres per individually analysed fish in the new sample was $3.32 \pm 0.34$. In fishes analysed in batches only $0.17 \pm 0.01$ fibre per individual fish was found (Figure 6.1; Online Supplement Table 3). If fibres originate from true ingestion, the abundance per individual fish should be similar in both individual and batch analysis. The significantly higher abundance in individually analysed fishes (Mann-Whitney-U-test $\mathrm{n}=$ 1002; $\mathrm{P}=0.001$ ) indicates strong secondary fibre contamination during sample processing. Per batch, 1.85 fibres had been counted, but the average batch contained the digestive tracts of 10.8 fishes. Exposure time during sample processing appears to be the major determinant of fibre abundance, not the number of fish. Control petri-dishes associated with individual fish had a $34 \%$ FO for fibres, with an average of 1.46 fibres per dish, whereas the control-dishes associated the batches had a $33 \% \mathrm{FO}$ with an average of 0.93 fibres. These \%FO's were not different in 2-sample z-tests $(\mathrm{P}=0.866)$ nor were the MannWhitney-U-tests on the number of fibres $(\mathrm{P}=0.70)$.

Subtracting control fibres in the range of 0.93 to 1.46 as observed in control dishes would considerably reduce fibre counts, but in addition there are many uncertainties about true comparability between control and sample results. When these corrections are applied to fibre counts per individual in batches (0.17 per fish) negative values would result. Although fibres are omnipresent in the environment, and thus likely occur also in fish stomach contents, we are unable to quantify them properly without special labourintensive precautions, and quantities are certainly very small. Consequently, it was decided to omit fibres from further analyses.

\section{Plastic Particle Properties}

For the dataset from Foekema et al. (2013), two particles were identified as polyethylene (PE), two as polypropylene (PP), one as polyethylene terephthalate (PET) and one as styrene acrylate (SA). The two items detected by Hermsen et al. (2017) consisted of poly- 


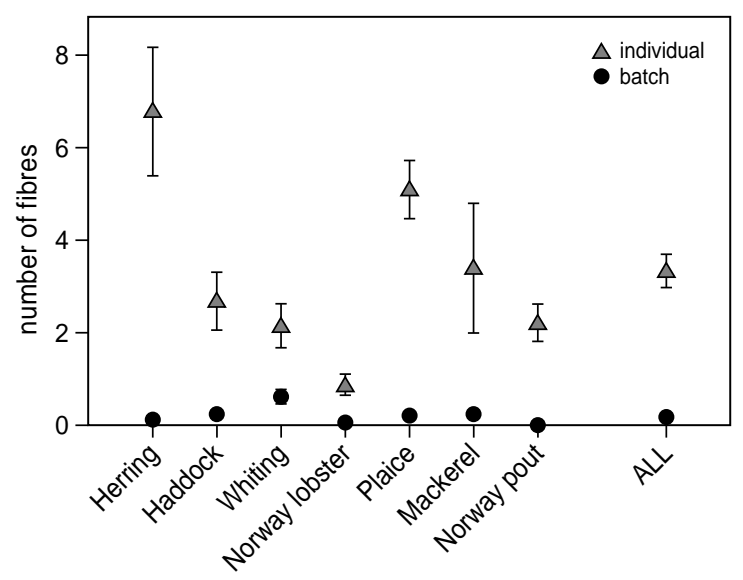

Figure 6.1. Average number of fibres \pm se per individual fish, detected in different fish species either assessed individually or in batches.

methyl methacrylate (PMMA). As expected, out of 69 items investigated with FTIR for the new data, a much-reduced number of 23 particles (33\%) were identified as being plastics. All other items in this subset were classified by FTIR as natural material, such as proteins, likely bits of fish bones or scales. Five of the plastic particles were certainly judged to be plastic by FTIR specialists but could not be reliably assigned to a specific polymer type, partly because the small size prevented proper cleaning or because they were black, which often hampers correct identification with FTIR. In the remaining part of the new study, visual assignment of particles as being plastic was based on the experience gained and identified 20 further items as plastic. Errors are expected to be minimal. Overall, in our combined study we report 96 particles as being plastic, of which 31 were identified by FTIR analyses (26 to specific polymer type), as detailed in Table 6.3. The category description of the plastic items was recorded for 56 items, most of them being sheets and fragments (41.6\%; Table 6.4).

Size measurements of non-fibrous plastic items were available for 35 of the 43 particles from the new dataset, 8 items were lost before a picture with size scale could be taken. Together with 42 items measured by Foekema et al. (2013) and two plastic particles from Hermsen et al. (2017), the average maximum diameter for all measured items $(n=79)$ was $3.18 \mathrm{~mm}$ (range $0.125-41.7 \mathrm{~mm}$ ) with two threads influencing the average particle size considerably (29 and $41 \mathrm{~mm}$ ). As described in the methods, the sieve meshes in different parts of our study were $0.2 \mathrm{~mm}, 0.02 \mathrm{~mm}$ and $0.03 \mathrm{~mm}$ respectively. Over the finer meshes, only one particle measured $0.125 \mathrm{~mm}$, next two up measured between 0.3 to $0.4 \mathrm{~mm}$, and all others were larger. From this, we expect that the loss of particles through the $0.2 \mathrm{~mm}$ sieve in the Foekema et al. (2013) study will be minimal and that its data can be integrated into the current overall analysis without risk for substantial bias in averages for overall particle numbers and sizes. 
Table 6.3. Composition of polymer types encountered in fish stomachs, shown as particle numbers (n) and percentages.

\begin{tabular}{llr} 
Polymer type & n & $\%$ \\
\hline PE polyethylene & 9 & 29.0 \\
PMMA polymethyl methacrylate & 5 & 16.1 \\
PP polypropylene & 4 & 12.9 \\
Acryl & 2 & 6.5 \\
PBM polybutyl methacrylate & 1 & 3.2 \\
PBT polybutylene terephthalate & 1 & 3.2 \\
PS polystyrene & 1 & 3.2 \\
PVC polyvinyl chloride & 1 & 3.2 \\
PET polyethylene terephthalate & 1 & 3.2 \\
SA styrene acrylate & 1 & 3.2 \\
Plastic of unknown detail & 5 & 16.1
\end{tabular}

Table 6.4. Plastic categories of items found ingested by fish, shown as particle numbers (n) and percentages.

\begin{tabular}{|c|c|c|}
\hline Category & $\mathrm{n}$ & $\%$ \\
\hline Sheet & 23 & 23.9 \\
\hline Fragment & 17 & 17.7 \\
\hline Probably industrial ${ }^{1}$ & 7 & 7.3 \\
\hline Thread & 5 & 5.2 \\
\hline Other ${ }^{2}$ & 2 & 2.1 \\
\hline Foam & 2 & 2.1 \\
\hline Unknown & 40 & 41.7 \\
\hline $\begin{array}{l}1 \text { microbead } \\
{ }^{2} \text { rubber }\end{array}$ & & \\
\hline
\end{tabular}

\section{Variables potentially influencing plastic abundance in North Sea fish}

Many variables may affect plastic ingestion, and despite a large sample size, there are relatively few fishes with plastic allowing statistically reliable analyses. The REML analysis (Table 6.5) shows that fish species and catch year are most often a significant variable, with latitude, season and distance to coast being less influential. Consequently, each of these factors are treated separately.

\section{Habitat}

The habitat types were compared with species belonging either to the demersal or pelagic feeding guild. Habitat was not included in the REML, as it is largely equivalent to species and Genstat does not allow that analysis. The difference in \%FO and the average number of plastics per fish when comparing demersal and pelagic fish are both significant when all species are included (Table 6.6). According to these results, demersal fishes are more prone to ingest plastics and contain more plastics than pelagic fish. However, as shown in Figure 6.2, cod influenced the pattern greatly. When removing cod from the analysis, the average particle number remains significantly higher in demersal species, but the \%FO is no longer significant. Comparing cod collected by Foekema et al. (2013) with new data from 2018, no significant difference was observed. This indicates both the reliability of the methods used in the different studies, and that cod generally ingests more plastics than the other fish species studied in the North Sea. As habitat may affect uptake of plastic in fish, it is considered in the following analyses of spatial and temporal distribution, to better understand how this may affect the data interpretation. 
Table 6.5. Results of Restricted Maximum Likelihood (REML) analyses showing different terms added and dropped and their associated significance results for all species combined, separated in their respective habitats (pelagic and demersal) and details on sprat and cod as these species influenced their respective habitat calculations. ' ${ }^{\prime}$ ', ‘*’) and ' $* * *$ ' indicate statistically significance with $\mathrm{P}=0.05,0.01,0.001$ respectively. 'n.s.' = not statistically significant. The variable tested is the number of plastics per fish.

\begin{tabular}{|c|c|c|c|c|c|c|c|}
\hline & ALL & $\begin{array}{l}\text { pelagic } \\
\text { fishes }\end{array}$ & $\begin{array}{c}\text { without } \\
\text { sprat }\end{array}$ & sprat & $\begin{array}{c}\text { demersal } \\
\text { fishes }\end{array}$ & $\begin{array}{l}\text { without } \\
\text { cod }\end{array}$ & $\operatorname{cod}$ \\
\hline Adding terms & $\mathrm{n}=4389$ & $\mathrm{n}=3229$ & $\mathrm{n}=1713$ & $\mathrm{n}=1516$ & $\mathrm{n}=1160$ & $\mathrm{n}=1046$ & $\mathrm{n}=114$ \\
\hline species & $* * *$ & ns & ns & & $* * *$ & ns & \\
\hline year & $* * *$ & ns & ns & $* * *$ & $* *$ & $* * *$ & ns \\
\hline latitude & $\mathrm{ns}$ & ns & ns & $* * *$ & ns & ns & ns \\
\hline season & ns & $* *$ & $* *$ & ns & ns & ns & \\
\hline coast & ns & ns & ns & $* *$ & ns & ns & ns \\
\hline \multicolumn{8}{|c|}{ Dropping terms } \\
\hline species & $* * *$ & ns & ns & & $* * *$ & $* * *$ & \\
\hline year & $* *$ & $*$ & $*$ & $* * *$ & $* *$ & $* *$ & ns \\
\hline latitude & ns & ns & ns & $* * *$ & ns & ns & ns \\
\hline season & $\mathrm{ns}$ & $* *$ & $* *$ & ns & ns & ns & \\
\hline coast & ns & ns & ns & $* *$ & ns & ns & ns \\
\hline
\end{tabular}

Table 6.6. Comparison of plastic uptake in demersal and pelagic species. Shown are sample numbers (n), the average number of particles per individual (avg $n$ ) including standard error $( \pm s e)$, the frequency of occurrence $(\% \mathrm{FO})$ and the according $95 \%$ Confidence Interval. First, all samples were compared and tested for significance (A), then cod was excluded as this species strongly influenced the data (B) and finally plastic uptake in cod was compared between earlier data by Foekema et al. (2013) and new data analysed for this study (C). Differences in average number particles were evaluated using the MannWhitney-U-test, and differences in \%FO tested by GLM of binomial proportions.

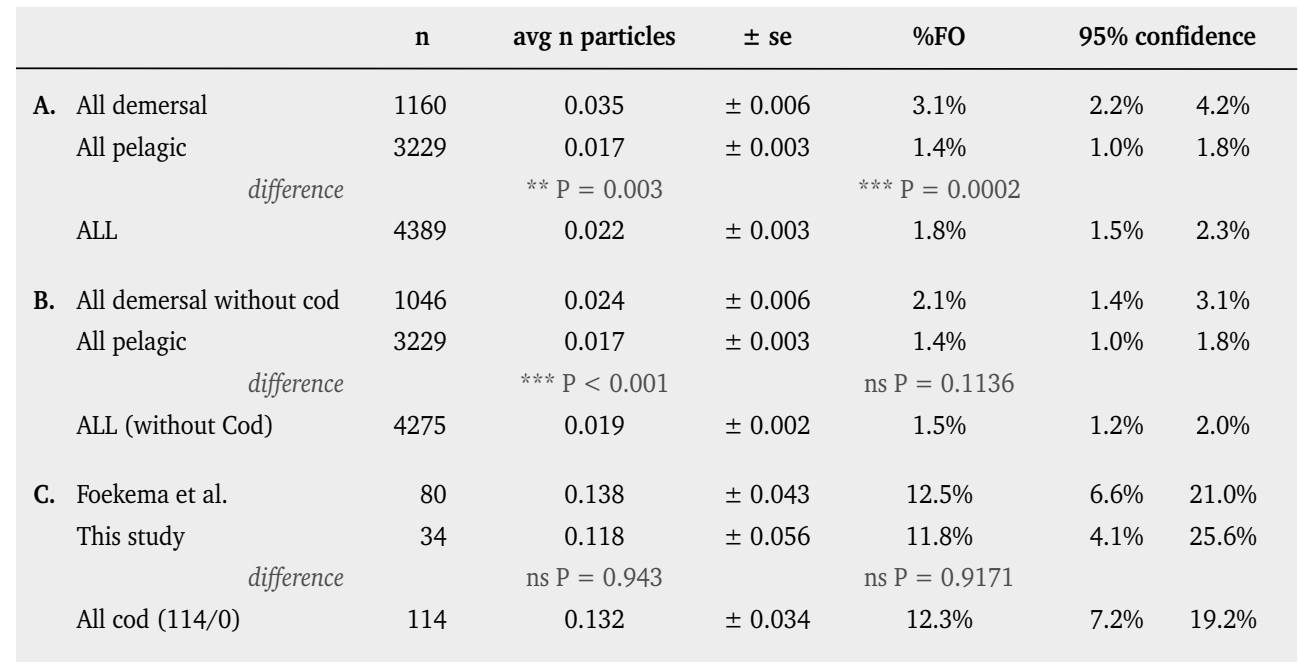




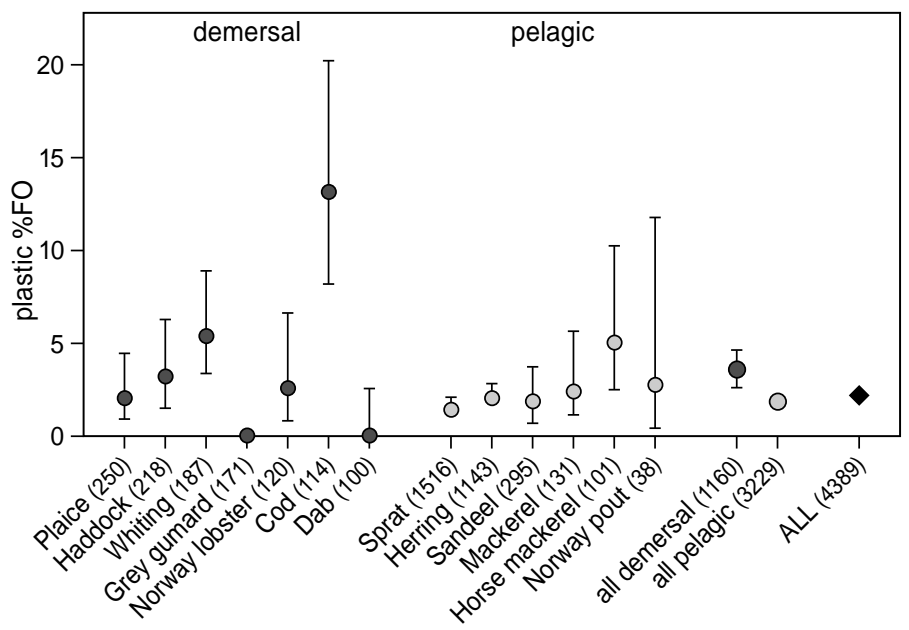

Figure 6.2. Observed frequency of occurrence of ingested plastic found in by different North Sea species with $95 \%$ confidence intervals and samples sizes shown in brackets with species names. Species are sorted according to their habitat and sample size. Data points for the small samples of anchovy and stickleback (Table 6.2) are not shown, but the data are included in the totals on the right side of the graph.

\section{Spatial distribution}

GLM analyses of binomial proportions of \%FO by latitude shows negative correlations (suggesting more fish with plastic at lower latitudes) for all tests (all species, demersal, pelagic, cod, herring), however none at a significant level. Only when singled out, sprat show a significant increase in the FO\% northwards ( $\mathrm{P}=0.035)$, which is opposite to the main correlation in the data. For demersal species linear regressions show negative correlations with latitude indicating a slightly higher average number of plastics at lower latitudes. For pelagic species this is the other way around suggesting slightly more litter at higher latitudes, including for sprat and herring separately.

When splitting data on the average number of plastic particles per fish into southern and northern fishes at $53^{\circ} \mathrm{N}$, the Mann-Whitney-U test indicates a significant difference for all species combined and pelagic species separately ( $\mathrm{P}<0.001$ more plastic north), overruling the tendency of demersal species to have more plastics further south. When making this split at $55^{\circ} \mathrm{N}$, it is herring that shows statistically more plastics at higher latitudes $(\mathrm{P}=$ 0.001 ). North of $55^{\circ} \mathrm{N}, 510$ herrings had an average of 0.0275 plastic particles per fish, while south of $55^{\circ} \mathrm{N}$ among 633 herrings, this was 0.0126 .

Distance to coast showed no clear correlation to plastic ingestion. In most cases the correlations in the linear regressions show a negative sign, possibly suggesting less plastics further offshore. However, this is not significant, not even in the numerous sprat samples.

\section{Temporal variation}

Fish samples were collected over a 10-year period between 2009 and 2018. The GLM over 
binomial proportions covering six datapoints show negative correlations, that is a declining frequency, for all species combined $(P=0.065)$, demersal species $(P=0.071)$ and significantly so for pelagic species $(\mathrm{P}=0.029)$. Sprat, with a significant downward trend at $\mathrm{P}<0.001$ strongly contributes to the pelagic trend. Except for sprat, our data lack sufficient numbers and yearly samples to allow meaningful statistics on individual species. A speculative trendline through the average number of pieces per fish (Figure 6.3) illustrates the suggestion of a more general decrease of plastic ingestion by North Sea fish over time. Graphs for demersal species and pelagic species separately have been provided in the Online Supplement. But except for sprat, no statistical evidence for declines are currently available.

Averaged over all species there is no clear seasonal trend. When cod (which had relatively more plastic, and was only sampled in winter) is left out, linear regression suggests a lower plastic ingestion towards winter months. However, GLM tests on proportions for summer, autumn and winter do not confirm this. Where none of the tests show seasonal differences for the demersal fish group, pelagic fishes show a decline in ingested plastic towards winter (GLM comparison of summer versus winter shows less plastic in winter at $\mathrm{P}=0.007$ ). Further tests indicated that this is caused by sprat (monthly change $\mathrm{P}<0.001$ and summer-winter difference $\mathrm{P}<0.001$ ).

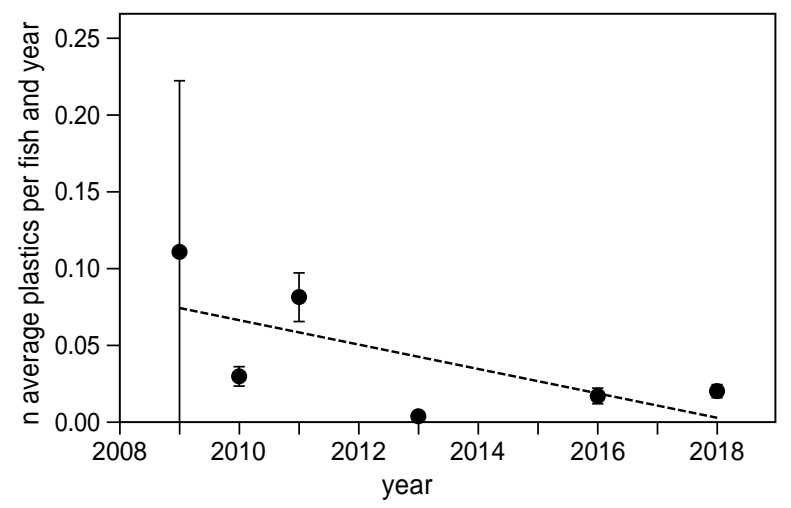

Figure 6.3. Average plastic particle number with standard error per fish by sampling year for all fishes sampled $(n=4389)$. The dotted line for a non-significant linear regression over these averages reflects decrease suggested by GLM analyses; for individual species such decline is significant only for sprat.

\section{Discussion}

This study focuses on the abundance of plastics in several fish species in the North Sea in a combination of existing datasets (Foekema et al. 2013; Hermsen et al. 2017), and a large set of additionally collected data. A detailed analysis of spatial and seasonal distribution is provided. 
When strict quality assurance is applied to avoid the risk of airborne fibre contamination, the \%FO and the average number of plastic particles per fish are generally low (Rummel et al. 2016; Wesch et al. 2017; Hermsen et al. 2018). As precautionary measures to prevent airborne fibre contamination could not always be applied, fibre abundance was analysed separately. Under current circumstances, the presence of fibres is impossible to quantify, but calculated corrections in most cases indicate fibres to be rare or absent. Airborne fibre contamination remains a complex issue. It is known that fibres deposit from the atmosphere to the ground or water surface and that they are available to organisms (Dris et al. 2016; Obbard et al. 2014; Bergmann et al. 2019). However, when rigorous contamination mitigation measures are applied, the frequency of occurrence of plastic in fish remains low in many studies (Hermsen et al. 2018), e.g. the study by Hermsen et al. (2017) was almost completely conducted under clean air conditions and showed zero fibres in 400 fishes and associated controls. Airborne fibre contamination without preventive measures has been studied previously. Kühn et al. (2018a) showed that stomach samples with the longest exposure time in air resulted in the highest number of fibres counted. By comparing results from individual fishes with those from batch samples, the current study supports this result by showing that exposure time has a bigger influence on the number of fibres than the number of individuals processed. From the controls, it is clear that most fibres originate from the surrounding air, rather than being ingested by the organisms. All the data of the current study as well as from previous studies show that a sensitive handling of fibre data is advisable. The exact description of mitigation methods applied can help in the future to disentangle the effects of secondary contamination on plastics ingested by organisms. For instance, Alomar \& Deudero (2017) and Alomar et al. (2017) mention fibres recorded in their control, but do not describe whether the amount of fibres were substracted or otherwise corrected for. Jabeen et al. (2017) combined fibres with threads (larger items originating from ropes or fishing nets), which makes it impossible to evaluate or compare data. We therefore recommend to record fibres and nonfibrous plastics separately and to use a size definition as proposed by Tanaka \& Takada (2016) to separate fibres from other threadlike materials fibres (diameter $<50 \mu \mathrm{m}$ ). Furthermore, all mitigation measures should be described in detail, including when and how they were applied. Controls should always be included whenever the sample is exposed to the air to avoid over-estimation of fibre ingestion in marine organisms.

Plastic ingestion in North Sea fish seems to exist at a low level, with 'only' $1.8 \%$ of fishes containing plastics and an overall average of 0.022 items per fish. Although low numbers of plastics were encountered in the digestive systems of fish, this does not exclude any presence of nano-plastics. Nano-plastics, ingested either directly or via prey, may be transferred from the gastro-intestinal tract (GIT) to organs and cells by passing e.g. the blood-brain barrier (Mattsson et al. 2017), with unknown consequences for health. Plastic ingestion in North Sea fishes is very low compared to some reports on non-fibrous plastic ingestion by fish species living in or near the North Pacific subtropical gyre (e.g. Boerger et al. 2010; Davison \& Asch 2011; Jantz et al. 2013). All species examined in this study had been investigated previously for plastic ingestion in the North Sea. However, the 
$\%$ FO varies greatly between the studies, depending on whether fibres were included or not (Murray \& Cowie 2011; Rummel et al. 2016; Lenz et al. 2016; Bour et al. 2018; McGoran et al. 2018; Welden et al. 2018). For non-fibrous plastics the numbers are comparable for most species. After correction for fibre contamination, Rummel et al. (2016) found $6.1 \%$ of 149 fishes from the North Sea to contain plastics. Nelms et al. (2018) analysed the stomachs of 31 mackerels and found 18 plastic particles in the stomachs, 13 of which were fibres. When only considering non-fibrous plastics, the analysed mackerel contained 0.16 pieces of plastics on average. For Norway lobsters in Irish waters, a high \%FO has been reported (Murray \& Cowie 2011; Welden \& Cowie 2016), including uncorrected numbers of fibres. Although many fibres in their samples consisted of fibre balls, making airborne contamination unlikely, it is unclear to what extent contamination might have occurred. Nevertheless, the \%FO of non-fibrous plastics is higher than found in the current study: Welden \& Cowie (2016) found non-fibrous plastics in a range of 3 up to $13 \%$ while the $\%$ FO in the Norway lobster sampled for this study remains at $2.5 \%$. In the current study and in Welden \& Cowie (2016) factors such as location have been found to significantly influence the results and may also play a role when comparing the results of the two studies. Almost half of the plastic items (41.9\%) identified in this study consist of polyethylene and polypropylene, the two most commonly produced plastic types (Geyer et al. 2017; PlasticsEurope 2018). Biofouling and vertical mixing can make these low density particles also available to demersal species (Kooi et al. 2017).

\section{Habitat}

The feeding habitat may play a role when looking at plastic uptake in different fish species. The current data indicate a higher $\% \mathrm{FO}$ and a higher average number of plastics in demersal species, whereby the \%FO is mainly influenced by the high uptake of plastics in cod. Rummel et al. (2016) also considered habitat as one of the factors that may influence the uptake of plastics in North Sea fishes and found pelagic fish to have a significant higher amount of plastics ingested than demersal species. However, their sample contained only 7 cods from one sample station, and none of them contained plastics.

\section{Trophic transfer}

Although trophic transfer of plastic from fish to seabirds and marine mammals has been documented (Hipfner et al. 2017; Hipfner et al. 2018; Nelms et al. 2018), the low number and small size of detected plastic in fish in the current study indicates that plastics from fish contribute little to the total amount of plastic ingested by seabirds such as the northern fulmar. In northern fulmars it is presumed that plastic particles smaller than 1-2 mm pass through the digestive system (Bravo Rebolledo 2011; Van Franeker \& Law 2015). Over the 2010-2014 period, 93\% of northern fulmars in the North Sea contained plastic. The average per bird was 33 plastic pieces, weighing $0.31 \mathrm{~g}$ togehter (OSPAR 2017). Although many of the fish species studied occur in fulmar diet, most items found in this study where small and once ingested (together with the prey fish), these items might not contribute substantially to the total mass of plastics in fulmars. 


\section{Spatial distribution}

Fulmar data on plastic ingestion indicate a decrease of plastics with latitude due to decrease in human population and maritime activity (Van Franeker et al. 2011; Kühn \& Van Franeker 2012; Trevail et al. 2015a). Foekema et al. (2013) found a significant lower level of plastics ingested in fish from northern latitudes. Combined with new data the results gain complexity. Demersal and pelagic fish tend to exhibit contrary correlations: demersal fish contained more and pelagic fish less plastics at low latitudes. The latitude of separating fish into north and south was considered to be most appropriate at $53^{\circ} \mathrm{N}$, where the North Sea flares from the Channel into an open basin. All species combined and demersal and pelagic species respectively contained more plastics north of $53^{\circ} \mathrm{N}$. This is in contrast to the original assumption and data by Foekema et al. (2013) but this may be influenced by species composition and fish numbers in the respective areas. One reason for this contrary result might be the sample of 960 sprat collected in 2013 in the southern region, whereas none of the individuals contained any plastics.

Litter in the North Sea is prevalent and widespread (Galgani et al. 2000; Gutow et al. 2018). Two studies from Chile and the UK both reported a higher uptake of plastic by fish closer to the coastline (Steer et al. 2017; Pozo et al. 2019). In this study, distance to coast remains unclear. The low $\% \mathrm{FO}$ and the skewness of this sample towards high sample sizes close to the coast may mask potential effects and even the large sample size of three combined datasets could not prevent difficulties in interpreting this data.

\section{Temporal variation}

From the REML the variation throughout years was revealed to be a strong factor influencing the uptake of plastics in fish. Over a 10-year period a decrease in the average number of plastics was observed. Sprat, the species with the largest sample size in this study, showed a strongly significant decrease of plastics in the \%FO, as well as in the average number of plastics. For all species combined, the decrease is reflected in the average number of plastic items per fish, but not significant regarding the \%FO. Plastic uptake in northern fulmars beached on the Dutch coast show a significant decrease in the period between 2002 and 2018 (Van Franeker \& Kühn 2019), and fish may reflect similar trends. However, it is clear that large sample sizes are necessary to ensure that other factors such as species, habitat and spatial distribution do not mask these results.

Plastic ingestion in fishes from different months and years was compared. More fish ingested more plastic items in summer. One reason for this difference could be the generally low uptake of natural food during winter in clupeids, gadoids and flatfish (Bowers \& Lee 1971; De Silva 1973; Schwalme \& Chouinard 1999; Möllmann et al. 2004). The effect of boreal seasons on the uptake of plastic in fish has not been studied extensively. Two studies from the Mediterranean Sea and eastern Canada recorded no seasonal correlation (Anastasopoulou et al. 2013; Liboiron et al. 2019), while McGoran et al. (2018) found a higher plastic uptake in fish during the summer months. 


\section{Suitable fish species for plastic monitoring}

In the framework of the MSFD, European countries are looking into options to develop further biota indicators in order to develop a broader monitoring system for plastic pollution in different ocean layers and plastic types in biota (Galgani et al. 2013a).

For the North Sea area, the northern fulmar has been assigned to monitor for three different critera set by the MSFD: (micro)plastics in biota (D10.C3), surface litter (D10.C1) and surface micro-litter (D10.C2). For the Mediterranean, Matiddi et al. (2017) recommend the use of loggerhead turtles (Caretta caretta) for plastic monitoring. For other regions where neither fulmars nor turtles occur, such as the Baltic Sea, no decisions for monitoring species have been made yet. Sampling plastics ingested by fish has been considered, but was classified as low level maturity in regards of sample protocol development (Galgani et al. 2013a). Criteria for suitable monitoring species are summarized by Galgani et al. (2013a). Species should have a broad distribution range, should regularly ingest plastics and should be sufficiently available for plastic ingestion studies. Further to this, the authors encourage the use of established monitoring frameworks for the collection of individuals. If possible, species should overlap in distribution ranges with other monitoring species to allow comparisons. Recently Bray et al. (2019) mention additional criteria specifically for fish, such as gut length as an estimator for retention time in fish, their commercial value and migration patterns.

Cod, as the only fish species with a $\% \mathrm{FO}>10 \%$, could be a potential candidate. Although this study found only small items of plastic in cod stomachs, anecdotal reports identified larger items in large cod, such as a soft drink can (Jawad et al. 2016) or a dildo (Sommers 2014). Its distribution range covers all North Atlantic coastlines from $36^{\circ} \mathrm{N}$ up to the Arctic Ocean (Froese \& Pauly 2019). Highly abundant in the North Sea, it also occurs in the southern and central parts of the Baltic Sea (Heesen et al. 2015). In the EU cod has a high commercial value: 143,782 tons of cod were caught in 2017 , equalling a market price of EUR 1.5 billion (EC 2018). Other than northern populations, the North Sea and Baltic cod do not exhibit large migration movements (Heesen et al. 2015; FAO 2019). Concurrently, sprat could be considered as the pelagic counterpart of cod. Similarly to cod, sprat has a broad distribution range, from northern Norway to the British Isles and to Morocco and also occurs in the Baltic, Mediterranean and Black Sea (Froese \& Pauly 2019). This distribution range allows comparison of other fish species in the Mediterranean. While some young sprat undertake inshore migrations in winter, adult sprat do not show large migration patterns. Sprat is only exploited locally, mainly for fishmeal production and on a small scale for human consumption (Heesen et al. 2015).

Through several fish stock monitoring surveys, cod and sprat are available in large amounts and can be included cost-efficiently to existing frameworks (Heesen et al. 2015). Gut length of both species could not be determined but retention time of plastic might be more related to gastric activity (Blier et al. 2007) and plastic morphology rather than gut length.

Monitoring cod would supplement the existing monitoring of plastic on seafloors by bottom trawling (MSFD indicator 10.1.2), which is already in an advanced state of matu- 
rity regarding implementation. Since the report by Galgani et al. (2013a), many studies have been published. Different methods of dissection and processing of fish have been developed and implemented in the North Sea (Lusher et al. 2013; Foekema et al. 2013; Collard et al. 2015; Steer et al. 2017; McGoran et al. 2017; McGoran et al. 2018) and to a lesser extent in the Baltic Sea (Rummel et al. 2016; Budimir et al. 2018) allowing the establishment of a standardised protocol.

\section{Conclusion}

The aim of this study was to evaluate plastics ingested by different fish species in the North Sea, including spatial and temporal patterns of plastic uptake. Data from three projects were combined to enlarge the sample size and improve the reliability of calculations. This study emphasises the necessity to treat fibres with high caution and separately from other plastic particles, as secondary contamination proves to represent major complications. The small amount of (non-fibrous) plastics in fish indicates that major impact of plastics on fish or predators are not likely. The low number of plastics do not make fish the ideal candidate for biota monitoring. However, as other more suitable species do not occur, fish may fill the gap and may add data on ecosystem niches that are currently not monitored. As variations occur, the advice is to set up sampling programs consistent in spatial and seasonal sampling.

\section{Acknowledgements}

We want to thank the (scientific) crews of all survey ships, contributing to the collection of the fish used in this study. Jenny Cremer helped with the GIS analysis. The authors are grateful to five anonymous reviewers for their valuable comments on the earlier version of this manuscript. Image credit for the graphical abstract belongs to NOAA fishwatch (fish; fishwatch.gov/api-tos) and Europe Map Vector from Vector.me (by vecteezy.com; North Sea map). S. Kühn is funded through the Joint Program Initiative (JPI) Oceans PLASTOX (Direct and indirect ecotoxicological impacts of microplastics on marine organisms) project by the Netherlands Organisation for Scientific Research (NWO) under the project number ALW-NWO 856.15.001. 
Plastic in North Sea fish 

Global and temporal variation of polymer types ingested by the northern fulmar and southern hemisphere relatives

$x=2$

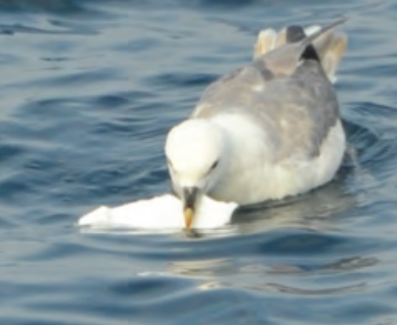

Susanne Kühn, Albert van Oyen, Elisa L. Bravo Rebolledo, Amalie V. Ask, Jan Andries van Franeker 


\begin{abstract}
Although ingestion of plastic by tubenosed seabirds has been regularly observed and quantified, identification of the polymer background of these plastics has been rarely described. Using known test materials, two identification methods (FTIR and NIR) were compared and both methods were found to be suitable for plastic identification. The match score threshold for correct identification should be set at least at $>80 \%$ to avoid misidentification of natural prey remains as being plastics.

Using NIR, the historical variability of plastics ingested by northern fulmars from the Dutch sector of the North Sea was analysed for three different periods since the 1980s. For the more recent decade, variability between fulmars from different regions in the north east Atlantic was investigated. Regional variation was further explored by analysing plastics obtained in the Antarctic from stomachs of southern hemisphere relatives of the fulmar and Wilson's storm-petrels. Results show that proportional abundance of polymer types in these seabirds is closely related to the plastic categories that they ingest (e.g. pellets, foam, fragments). The uptake of different plastic categories and related polymer types most likely reflects the production and disposal fluxes on a global scale.
\end{abstract}




\section{Introduction}

Tubenosed seabirds (Procellariiformes) are known to ingest debris including plastics from the sea-surface. At a global scale, at least 60\% (84 of 141) procellariform seabirds species have been recorded to ingest plastic (Kühn et al. 2015). Ingestion of plastic might occur accidentally as e.g. in albatrosses that forage on eggs of flying fish attached to pieces of floating plastics (Pettit et al. 1981) or by foraging on prey that ingested plastics itself (Hipfner et al. 2017). In contrast, intentional ingestion is suspected in many other seabird species with a less specialized diet (Ryan 1987). The effects of plastic on marine wildlife are largely unknown due to the many factors that might influence the level of harm. These factors include uptake, retention time, digestion mode of different organisms but also the broad variety of polymers, shape, colour and chemical burden of the plastics themselves. Retention time of plastic is difficult to determine in free-ranging seabirds, it depends on the shape and the size of the ingested plastic in relation to body size, the wearing process in the stomach and the threshold size that is needed to excrete the plastic (Ryan 2015a). Studies indicate a loss of 75\% of the plastic load per month (Van Franeker \& Law 2015) or a complete loss of particles in less than two months (Terepocki et al. 2017) up many moths (Ryan \& Jackson 1987; Ryan 2015a). Ingested plastics may cause mechanical disruptions and a false feeling of satiation (Kühn et al. 2015). Chemical mixtures of substances added during the manufacturing process of polymers and substances adsorbed from the marine environment are of concern, as the potential toxic substances may harm marine organisms (Oehlmann et al. 2009; Teuten et al. 2009; Rochman 2015; Tanaka et al. 2015). However, the extent of potential harm remains unclear, due to the many other factors influencing the uptake of chemicals in marine wildlife (Werner et al. 2016).

Plastic ingested by seabirds has been recorded from the 1960s onward (Threlfall 1968; Kenyon \& Kridler 1969), an increasing body of publications proves that plastic and the ingestion of it by marine megafauna occurs over all the world's oceans (Provencher et al. 2017). In recent years, research quantifying the abundance of plastics in organisms (frequency of occurrence, average number and sometimes average mass of plastic items), have been complemented by investigations of polymer types and related chemical burdens. This is related to the technical progress in analysis methods such as infrared and mass spectroscopy and to the small size of plastics that require advanced identification techniques. Studies investigating plastic ingestion by fish are relatively recent and frequently use Fourier Transform Infrared spectroscopy (FTIR) or Raman spectroscopy to identify polymer types (e.g. Löder \& Gerdts 2015; Lusher et al. 2013; Rummel et al. 2016; Pellini et al. 2018; Wieczorek et al. 2018; Kühn et al. 2019). However, except for more incidental reports, the identification of plastic polymers is still relative scarce in marine megafauna (e.g. Yamashita et al. 2011; Amélineau et al. 2016; Avery-Gomm et al. 2016; Tanaka et al. 2019, Eriksson \& Burton 2003; Van Franeker et al. 2018; Pham et al. 2017; Rizzi et al. 2019). Data on the composition of polymer types can be useful, when discussing potential toxic consequences of plastic ingestion, as different plastic types can be related to different substances added to the polymer (Lithner et al. 2011). Spectroscopy 
produces light reflection or transmission spectra that can be compared to a library of known polymer spectra. The match between spectra is often expressed in percentages, however the threshold of acceptance of the results, as being reliable, differs in many studies. Therefore a first aim of this study was to identify a suitable threshold level for the reliability of infrared spectroscopy results. We did this in a comparison of results from Near Infrared spectroscopy (NIR) and FTIR of a set of known polymer and natural particles.

Since the 1980s plastic ingestion has been observed in northern fulmars (Fulmarus glacialis; Van Franeker 1985). From 2002 onwards, this procellariform seabird has been used as a monitoring tool for marine debris for the Olso-Paris Convention for the Protection of the Marine Environment of the North-East Atlantic (OSPAR) in the North Sea. The fulmar belongs to one of the best studied species with regards to the quantification of plastic pollution and has been proven to be a suitable monitoring tool to assess changes in abundance and types of plastic (Van Franeker et al. 2011; Van Franeker \& Law 2015; OSPAR 2017). Research has been expanded from the Netherlands to the entire North Sea area and further to the North Atlantic, including the Faroe Islands (Van Franeker et al. 2011), Iceland (Kühn \& Van Franeker 2012) and Svalbard (Trevail et al. 2015a). Based on a common standardized protocol (Van Franeker 2004; Van Franeker et al. 2011; OSPAR 2015) these results are easily comparable to other studies and study regions, such as the Canadian (sub)Arctic (Mallory 2006; Mallory 2008; Provencher et al. 2009; Avery-Gomm et al. 2018) and the North Pacific Ocean (Donnelly-Greenan et al. 2014; Nevins et al. 2005; Avery-Gomm et al. 2012; Terepocki et al. 2017). So far, the outcomes of these studies report the frequency of occurrence, average number of pieces and plastic mass.

This study aims to deepen the knowledge of plastics ingested by northern fulmars and a number of its relatives from the Southern Ocean from quantification towards more information regarding ingested polymer types. NIR was used to evaluate potential differences in ingested polymers on a temporal and spatial scale. Using stored samples from long-term fulmar studies, the polymer composition of plastics from stomachs of fulmars from different time periods in the North Sea were analysed. In order to consider regional differences, North Sea results were compared to the polymer composition of plastics from fulmars from other locations in the northeast Atlantic Ocean (Faroe Islands, Iceland and Svalbard) and to plastics obtained from a number of related species in the Southern Ocean.

\section{Methods}

\section{NIR method evaluation}

For this study, in order to process a large series of plastic particles relatively quickly, we applied NIR analysis using a handheld Phazir (DTS-PHAZIR-1624). NIR is not used regularly in identifying polymers ingested by seabirds (but see: Yamashita et al. 2011). To evaluate the quality of NIR assessments for our type of work on materials from seabird stomachs, we prepared a comparative experiment applying NIR and FTIR on a selection of 189 
plastic and natural items. The composition of this sample reflects a broad variety of items potentially encountered in stomachs of marine organisms, both natural prey (remains) and marine debris items. A total of 111 plastic items with known polymer composition, including 18 different plastic types, of which 5 so-called bioplastics were prepared. All plastics had varying colours and belonged to different plastic categories (pellets, sheets, threads, foam and fragments, according to Van Franeker et al. (2011)). Additionally, 78 natural items were included, including prey items such as fish bones and otoliths, eye lenses of squid and fish, crab carapaces and shells of bivalves and insects, skin, bill structures and feathers of different seabirds, gastropods but also natural non-food items such as stones, wood, wool, seaweed and seeds. The details of each item can be found in the Online Supplement Table 1.

All items were analysed with both, NIR (DTS-PHAZIR-1624 for 1600-2400 nm library details: Online Supplement Table 2) and FTIR (ATR-FTIR; Shimadzu Prestige 21, 10 Scans, Library ATR Polymer2, IRs Polymer 2, T-Polymer). In a first step, the results of NIR and FTIR were collated to see whether both methods delivered comparable and reliable results.

In a second step, threshold values for the reliability of the results were identified. In infrared spectroscopy results are usually expresses in percentages, as a match score. The higher the percentage given, the more overlap between sample and library spectrum has been found. In literature different thresholds for these match scores are used to describe the reliability of infrared spectroscopy results. For this study, the reliability of infrared spectroscopy plastic identification was tested at the thresholds of 70, 80 and $90 \%$, based on the 189 known polymers and natural items.

All measurements were judged on their reliability, they were called reliable when:

1. The correct material was assigned to the particle measured according to the actual match threshold;

2. Natural materials were either described as natural or reached less than the assigned match threshold, meaning their identification as polymer was not successful.

Polymers identified by FTIR as HDPE and LDPE, were grouped as PE, as the NIR library did not allow such a detailed analysis.

\section{Seabird samples}

For this study, we analysed plastics ingested by northern fulmars (Fulmarus glacialis), three of its Southern Ocean close relatives (southern fulmar Fulmarus glacialoides, cape petrel Daption capense, and snow petrel Pagodroma nivea) plus the Wilson's storm-petrel (Oceanites oceanicus). For the temporal analysis of, plastics from beached fulmars in the Netherlands a selection of 129 birds covering three decennia (1980-2011) was used. For a regional comparison between fulmars from the north Atlantic, stomach contents of northern fulmars from the Netherlands were compared to samples from the Faroe Islands, Iceland and Svalbard. Samples from Antarctica were collected during research expeditions between 1984 and 1998 in the Windmill Islands area near the Australian Casey station $\left(66^{\circ} \mathrm{S}, 110^{\circ} \mathrm{E}\right)$ in eastern Antarctica. Overall, our study used plastics from stomachs of 317 individual birds, as specified in Table 7.1. 


\section{Dissection protocol}

All birds were dissected according to guidelines by Van Franeker (2004) and OSPAR (2015). In short, external measurements and details on moult were recorded. Internally, the sex, age, organ health and condition were assessed and the stomachs were removed. The stomach content was sieved on $1 \mathrm{~mm}$ mesh size and all hard items were sorted under a stereomicroscope as either natural food or plastics. The plastic items were further split into plastic categories: industrial pellets, microbeads, sheet, thread, foam, fragments and 'other' plastics. All details regarding frequency of occurrence, average plastic number and mass were published within earlier publications (Van Franeker \& Bell 1988; Van Franeker 1985; Van Franeker et al. 2011; Kühn \& Van Franeker 2012; Trevail et al. 2015a).

\section{Sample analysis}

All plastic items encountered in the seabirds were individually weighed on a Sartorius electronic scale to an accuracy of $0.0001 \mathrm{~g}$. NIR analysis was conducted using a handheld near infrared spectroscope with integrated spectrum library containing 28 different polymer types (for details see Online Supplement Table 2). Results were accepted based on the match score threshold level identified during the NIR/FTIR experiment. The 10 most occurring polymer types are given in full detail. Other polymer types had a very low occurrence throughout the total sample ( $<15$ particles, each type representing $<0.3 \%$ ) and were combined in the category 'other'.

\section{Data analysis}

For visual impressions, graphs are presented as stacked columns either for numbers or mass. As mass of plastic is considered more important in terms of potential harm (Van Franeker

Table 7.1. Available sample of procellariform seabirds, including details on location and collection year.

\begin{tabular}{|c|c|c|c|}
\hline Species & Country & years & $\mathrm{n}$ birds \\
\hline Northern Fulmar & Netherlands & 1982-2011 & 129 \\
\hline Northern Fulmar & NL1980-89 & $1980-89$ & (58) \\
\hline Northern Fulmar & NL1909-99 & $1990-99$ & (32) \\
\hline Northern Fulmar & NL2010-11 & 2010-19 & (39) \\
\hline Northern Fulmar & Faroe Islands & 2011 & 50 \\
\hline Northern Fulmar & Iceland & 2011 & 46 \\
\hline Northern Fulmar & Svalbard & 2013 & 35 \\
\hline NORTHERN FULMAR & TOTAL & 1982-2013 & 260 \\
\hline Wilsons Storm-petrel & Antarctica & 1986-98 & 45 \\
\hline Cape Petrel & Antarctica & $1985-86$ & 9 \\
\hline S. Fulmar & Antarctica & 1984 & 2 \\
\hline Snow Petrel & Antarctica & 1985 & 1 \\
\hline ANTARCTICA & TOTAL & 1984-98 & 57 \\
\hline
\end{tabular}


et al. 2011; Provencher et al. 2019) and is used in the framework of monitoring of plastics in fulmars (OSPAR 2015) most graphs depict compositions mass percentages. However, all underlying data (numbers and mass) are provided in Online Supplement Table 3.

Tests for statistical significance in polymer type proportions were conducted with https://epitools.ausvet.com.au/ as recommended by Provencher et al. (2017). We compared sample proportions of polymer types with 2-sample z-test (two-tailed, significance level set at $\mathrm{P} \leq 0.05$; https://epitools.ausvet.io/ztesttwo). This test is only suitable for plastic numbers and therefore all tests refer to numbers, rather than to the mass of plastics.

\section{Results}

\section{NIR method evaluation}

A total of 189 items were analysed with both NIR and FTIR. For known polymer samples, FTIR scored slightly higher than NIR. However, FTIR failed more often in the recognition of natural materials. At a match score of $>90 \%$, FTIR identified $32 \%$ of all natural items falsely, usually as being PA. With lower match scores, the percentage of false FTIR classifications for natural materials increased to $43 \%$ and $46 \%$ for thresholds of $>80 \%$ and $>70 \%$ respectively. Data in Table 7.2 indicate, that for FTIR a high threshold level is necessary to avoid misidentification, especially in studies of seabird stomach contents. NIR identified less polymers correctly than FTIR but was more reliable in distinguishing plastics and natural items.

Yamashita et al. (2011) described difficulties with measuring dark items, when using NIR and the same was experienced during this study. At a threshold level of $80 \%$, NIR only identified less than half of the dark particles (48\%) while FTIR scored $76 \%$ of the items correctly. Light-coloured particles were identified correctly in similar percentages of $72 \%$ and $69 \%$ of the cases for NIR and FTIR respectively.

From Table 7.2 it appears that in NIR, the match score does not have a major influence on the reliability of the outcome of synthetic polymers, but a strong influence on the recognition of natural materials. Almost all natural materials are identified as such when a threshold of $>80 \%$ match score is applied. We recommend to set the threshold for both methods at least at $>80 \%$. The $>80 \%$ threshold will therefore be used in the further description of results for this study.

Table 7.2. Percentages of correctly identified material for NIR and FTIR respectively, when different match score thresholds were applied.

\begin{tabular}{lccccccc} 
& n pieces & FTIR $>70 \%$ & FTIR $>80 \%$ & FTIR $>90 \%$ & NIR $>70 \%$ & NIR $>80 \%$ & NIR $>90 \%$ \\
\hline Polymers & 108 & $66.7 \%$ & $70.4 \%$ & $77.8 \%$ & $67.6 \%$ & $67.6 \%$ & $68.5 \%$ \\
Natural & 81 & $45.7 \%$ & $46.9 \%$ & $67.9 \%$ & $84.0 \%$ & $98.8 \%$ & $100.0 \%$ \\
\hline All items & $\mathbf{1 8 9}$ & $\mathbf{5 7 . 7} \%$ & $\mathbf{6 0 . 3} \%$ & $\mathbf{7 3 . 5} \%$ & $\mathbf{7 4 . 6} \%$ & $\mathbf{8 1 . 0} \%$ & $\mathbf{8 2 . 0} \%$
\end{tabular}




\section{Plastic in procellariform seabirds}

A total of 5303 plastic pieces were analysed by NIR. To evaluate the impact of using the $>80 \%$ spectrum match, Figure 7.1 shows results when no restriction is applied and when the $70 \%, 80 \%$ or $90 \%$ match thresholds are used. PE and PP identification slightly gain importance when higher thresholds are applied, indicating that spectra for less common polymers show less similarity to those in the polymer library. Using the $>80 \%$ spectra

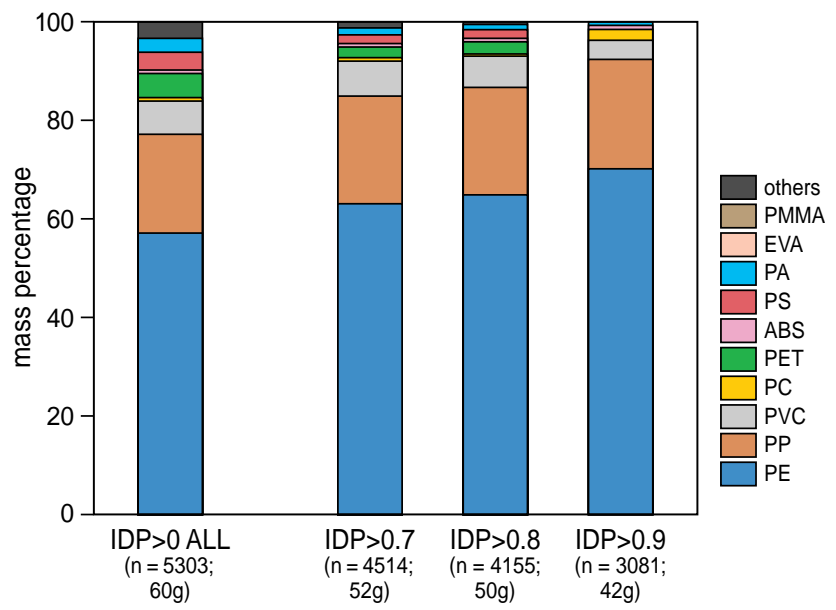

Figure 7.1. Polymer identification with NIR, applying different match score thresholds of $>70,>80$ and $>90 \%$ (shown as identification probabilities - IDP). Proportions percentages are shown in terms of mass for all plastic items encountered in all seabirds used for this study.

Table 7.3. Categories of marine debris items collected from all birds used in this study.

\begin{tabular}{|c|c|c|c|c|c|c|c|c|c|}
\hline Species & Country & $\mathrm{n}$ plastic & fragment & pellet & sheet & thread & foam & other & bead \\
\hline Northern Fulmar & Netherlands & 3439 & 1252 & 650 & 657 & 190 & 579 & 111 & 0 \\
\hline N. Fulmar & NL1980-89 & (778) & 213 & 356 & 69 & 43 & 89 & 8 & 0 \\
\hline N. Fulmar & NL1909-99 & (1129) & 389 & 94 & 378 & 83 & 97 & 88 & 0 \\
\hline N. Fulmar & NL2010-11 & (1532) & 650 & 200 & 210 & 64 & 393 & 15 & 0 \\
\hline N. Fulmar & Faroe Islands & 536 & 361 & 36 & 49 & 35 & 50 & 4 & 1 \\
\hline N. Fulmar & Iceland & 342 & 199 & 14 & 39 & 25 & 55 & 1 & 9 \\
\hline N. Fulmar & Svalbard & 595 & 428 & 16 & 47 & 76 & 24 & 3 & 1 \\
\hline N. FULMAR & TOTAL & 4912 & 2240 & 716 & 792 & 326 & 708 & 119 & 11 \\
\hline Wilson's Storm-petrel & Antarctica & 342 & 194 & 110 & 14 & 2 & 20 & 0 & 2 \\
\hline Cape Petrel & Antarctica & 37 & 15 & 11 & 0 & 1 & 10 & 0 & 0 \\
\hline S. Fulmar & Antarctica & 11 & 10 & 1 & 0 & 0 & 0 & 0 & 0 \\
\hline Snow Petrel & Antarctica & 1 & 0 & 0 & 0 & 0 & 1 & 0 & 0 \\
\hline ANTARCTICA & TOTAL & 391 & 219 & 122 & 14 & 3 & 31 & 0 & 2 \\
\hline ALL BIRDS & TOTAL & 5303 & 2459 & 838 & 806 & 329 & 739 & 119 & 13 \\
\hline
\end{tabular}


match threshold, 4155 of plastic pieces and 50 grams of plastic mass were identified to a specific polymer type. Thus, 1148 plastic pieces (10 grams) remained below this threshold and are therefore not included in the further analyses of plastic type proportions.

Plastic items belonged to seven different categories (Table 3). Of the 5303 items, $19.8 \%$ could not be assigned to a specific polymer when a spectrum match threshold of $>0.8$ was applied. As already indicated during the testing phase, NIR had difficulties with identifying foams, of the 739 foam items measured, only $42.5 \%$ could not be identified. Even though the identification of threads scored high in the testing phase $(77 \%$ were correctly identified), in more than half of the threads measured in bird stomachs (53.8\%) the polymer type remained unclear.

The polymer composition of the 4155 plastic particles that were successfully identified, is shown in Figure 7.2, both in terms of numbers and mass. Data shown in Figure 7.2 clearly indicate a relationship of plastic category and polymer types. Pellets and fragments mainly consist of PE (79 and $72 \%$ in terms of mass, respectively), while the other categories show

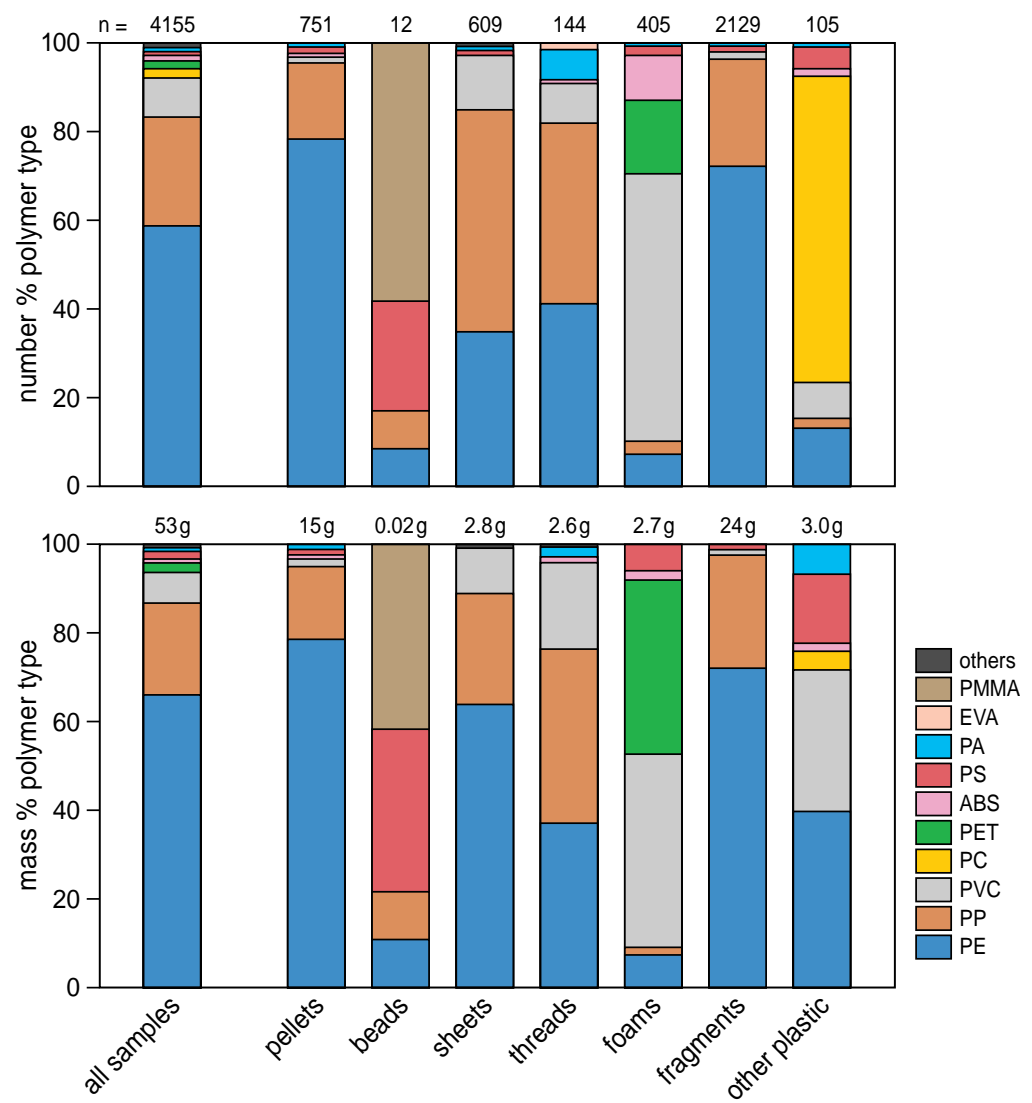

Figure 7.2. Polymer type proportions of all items identified in this study, belonging to different plastic categories. Top: polymer type proportions in numbers. Bottom: polymer type proportions in gram. Graph is based on data shown in Online Supplement Table 3.2a. 
more variation. Threads for example consist of 37\% PE, 39\% PP and 20\% PVC. Microbeads in contrast were mainly made of PMMA (42\%) and PS (37\%) but due to their low abundance do not strongly influence overall results.

As the variation between plastic categories is substantial, spatial and temporal comparisons are presented separately per plastic category as well.

\section{Temporal comparison}

Plastic in fulmars from the Netherlands have been studied from the 1980s onwards. Here we compare plastics ingested by northern fulmars from three decennia. Pellets comprised the majority of items (54\%) in terms of mass during the 1980s (Figure 7.3). In the 1990s, the mass of fragments, sheet and threads increased and consequently the proportion of pellets decreased (20\%). However, in the 2010s pellets again gained relative importance (32\%) together with fragments (40\%) and foam (13\%), possibly influenced by a fulmar from 2010 with an unusual number of pellets $(\mathrm{n}=72)$. In the $1980 \mathrm{~s}, 85 \%$ of the plastics were made of PE, $11 \%$ of PP and only $4 \%$ were made of other polymer types (Figure 7.3).
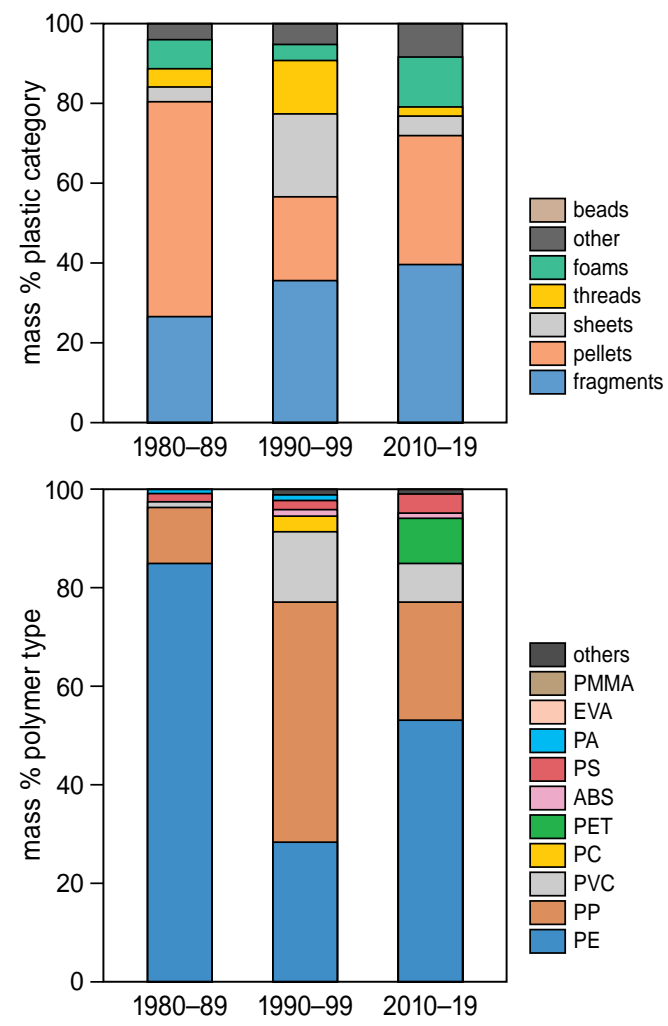

Figure 7.3. Top: Plastic categories ingested by northern fulmars in three decennia. Bottom: Polymer categories of these plastics. Both graphs show proportions in terms of mass. Graph is based on data shown in Online Supplement Table 3.3a \& b. 
In the 1990s more diversity could be observed, with $14.4 \%$ of PVC and $2.9 \%$ PC. PP comprises of almost half of the plastics (49\%). In the most recent decennia, PE gained relative importance (53\%), while PC almost disappeared (0.3\%). PET and PS increased (9.4 and 7.9\% respectively). Numbers of PE differ significantly for all three decennia $(\mathrm{P}<0.0003)$.

\section{Regional comparison North Atlantic}

Data for northern fulmars is available for four regions in the North Atlantic: the Netherlands, the Faroe Islands, Iceland and Svalbard. For the Netherlands, only data from the most recent decennia (2010-2019) was included, as data from the other regions were collected between 2011 and 2013. Fragments are the most dominant plastic category in all regions, for the Faroe Islands and Iceland, more than half of the plastic mass consists of fragments (Figure 7.4). Pellets are more common in the Netherlands, but decrease with higher latitude. Threads are comparably abundant in Iceland and Svalbard. The highest variation of plastic types can be found in the Netherlands. PE and PP comprise the majority of plastic mass in all regions and increase with latitude, with the exception of
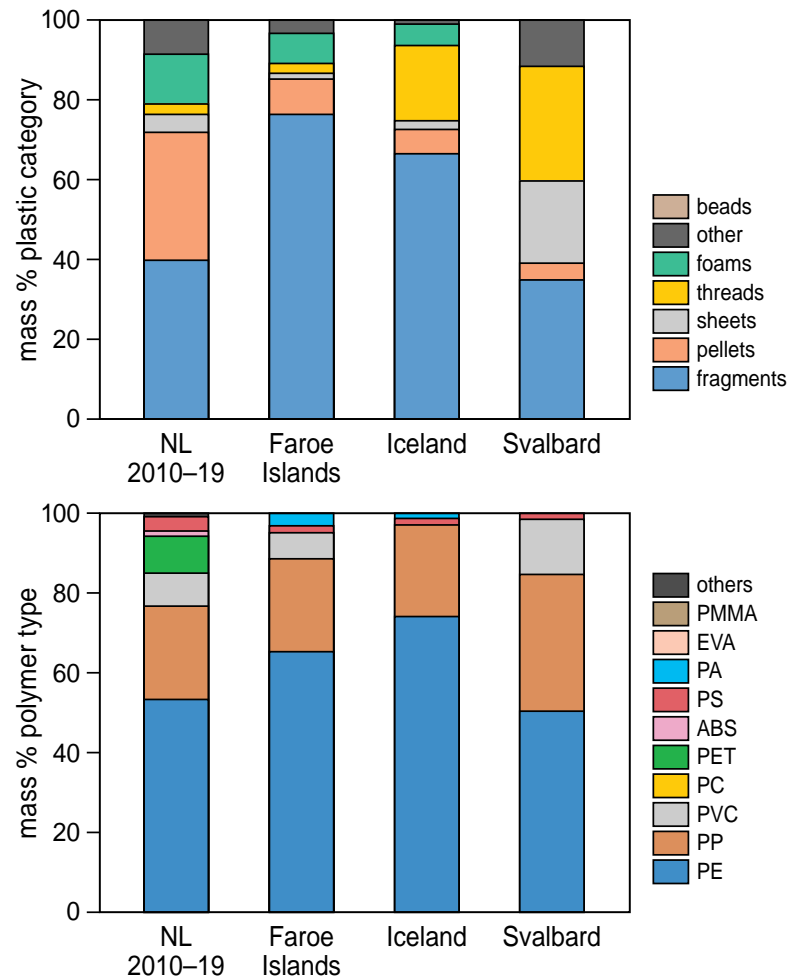

Figure 7.4. Top: Plastic categories ingested by northern fulmars from four North Atlantic regions. Bottom: Polymer categories of these plastics. Both graphs show proportions in terms of mass. Graph is based on data shown in Online Supplement Table 3.4a \& b. 
Svalbard, where the proportions of PE mass seems to closer to those of the Netherlands (Figure 7.4). However, when testing for significant differences in PE numbers, the Netherlands have significantly less $\mathrm{PE}$ plastic items than all other locations $(\mathrm{P}<0.0001)$. The Faroe Islands, Iceland and Svalbard do not show significant differences in the number of PE items ( $\mathrm{P}>0.1$ ). Noticeable PVC comprises $13 \%$ of mass on Svalbard but much lower proportions in the Netherlands, the Faroe Islands and Iceland (8, 6 and $0 \%$ respectively).

\section{Global comparison}

Sample numbers for the southern species are, except for the Wilsons's storm-petrel, very low. There was one snow petrel available that had ingested one item (which could not be identified at $>80 \%$ match score). Figure 7.5 shows the different plastic categories found in northern fulmars and in Antarctic seabird species. The variation in northern fulmars is much higher with more sheets, thread and other plastic, while the Antarctic samples are dominated by fragments and pellets. In Figure 7.5, the polymer composition (in mass) for the southern species separately, and as a group are shown. In terms of numbers, the
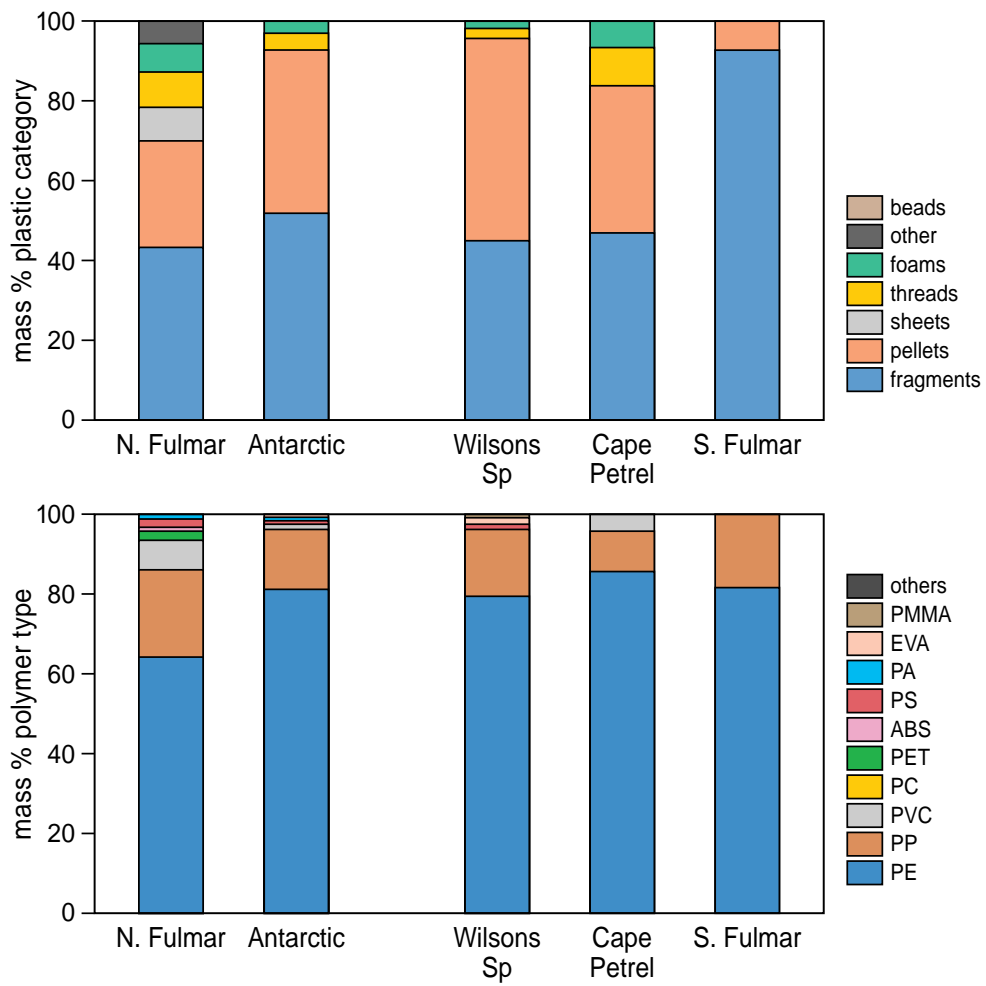

Figure 7.5. Top: Plastic categories ingested by northern fulmars from the North Atlantic and related seabird species from the Southern Ocean (both all southern species combined and separately per species). Bottom: Polymer categories of these plastics. Both graphs show proportions in terms of mass. Graph is based on data shown in Online Supplement Table 3.2b (N. fulmars) and 3.5a \& b. 
polymer composition of the three remaining southern species does not differ significantly ( $P>0.73$ ) from each other and therefore these species can be treated as one group. In southern species PE mass is higher (81\%) than in northern fulmars (64\%) (Figure 7.5). Also, the number of PE items is significantly higher in Antarctic birds than in northern fulmars $(\mathrm{P}<0.0001)$.

\section{Discussion}

For this study, NIR spectroscopy was compared with the more commonly used FTIR and a threshold for the correct identification of polymers is recommended. Plastics ingested by northern fulmars and some procellariform seabird species from Antarctica were analysed using infrared spectroscopy. Temporal and spatial comparisons were made, covering three decennia and different regions of the North Atlantic and the Southern Ocean.

\section{Infrared spectroscopy thresholds}

FTIR analysis is a method increasingly used to identify plastics ingested by marine organisms. Even though FTIR analysis has been applied mainly to fish studies (Online Supplement Table 7.4), where items are too small to identify by visual inspection solely (Lusher et al. 2017b), the interest in polymer description and related chemical burden in marine megafauna is growing (Provencher et al. 2017). Currently, there is no common agreement on match score threshold levels in IR analysis. Literature research revealed 61 studies that have used either FTIR or NIR to determine the composition of plastics in seabirds, marine mammals, turtles and marine fish (Online Supplement Table 4). The threshold varied between 60 and 93\%. Most studies that give details have varied in acceptance rate between 70 and 85\% (Alomar \& Deudero 2017; Bessa et al. 2018; Ory et al. 2017; Ory et al. 2018; Tanaka \& Takada 2016). Unfortunately, a majority of studies ( $\mathrm{n}=$ 37) using FTIR, did not provide any details on match score thresholds at all. Therefore, it is unclear, whether they used a threshold or rather accepted any result displayed. For this study a threshold level was established for the reliable identification of plastic items ingested by marine organisms, using either FTIR or NIR. By testing both methods on a series of various natural and plastic items, we conclude that at least a match score of $80 \%$ should be achieved. This is a slightly higher threshold level than proposed by Lusher et al. (2017b), who recommended the (often arbitrary) average threshold levels used in previous studies (70-75\%) as a standard.

Many different natural items such as fish and squid lenses, beaks, bones, skin and feathers were falsely identified by FTIR as being polymers. Precaution should be taken to avoid overestimation of plastics ingested by organisms, when solely relying on FTIR outcomes. Good background knowledge of typical natural food remains, occurring in the species studied, is highly recommended. Although NIR has shown to have difficulties with foams and dark colours, still both FTIR and NIR seem to be reliable and can be recommended for the identification of polymers. 


\section{Polymer types in seabirds}

IR polymer analysis has not been applied to any of the species researched in this study. Although plastic is common in these species, the only crude polymer identification in Wilson's storm-petrels and northern fulmars has been conducted by Moser \& Lee (1992), using density separation. In both species the great majority of ingested plastic items were floating, and, according to the authors, belonged to either PE or PP. This overlaps with the results from the current study, where $\mathrm{PE}$ and $\mathrm{PP}$ were the most common polymer types in both, time and space.

Our data indicate, that plastic categories determine polymer characteristics more than time- and space scale. Characteristics of plastics ingested by birds, such as size and shape, have changed through time (Ryan 2008; Van Franeker \& Law 2015). Fragments are predominantly made of PE (71\%) and PP (27\%). When fragment mass is high in birds, PE and PP are the most abundant polymer type as well. Many pellets in a sample increase the relative PE mass, e.g. in early data from the Netherlands (46\%) and in Antarctic seabirds (31\%). Since the 1990s, pellets decreased in northern fulmars, while mainly fragment mass increased (Van Franeker \& Law 2015) and also PE mass decreased in the same time. Unfortunately, no recent data of plastic ingestion by Antarctic seabirds is available that could confirm similar trends in the Southern Ocean. However, two recent studies from the Southern Ocean, investigating microplastics at the water surface, found PE and PP to be the dominant polymer types in non-fibrous plastics (Cincinelli et al. 2017; Isobe et al. 2017).

Threads were mainly found in Iceland and Svalbard, both remote places, where fisheryrelated plastic dominates the litter found on beaches (Bergmann et al. 2017a). The majority of threads (in terms of mass) ingested by all northern fulmars in the North Atlantic were made of PE (49\%) and PP (21\%). Only 3\% of ingested threads comprised of PA (Online Supplement Table 3.3b), probably explained by the high density of PA, causing it to sink out of reach of surface-foraging seabirds. Soft materials such as sheets and foam are less abundant in birds from the Southern Ocean (Figure 7.6). Seabirds migrate from more polluted areas to Antarctica (Van Franeker \& Law 2015). Soft material digests quicker than hard pellets or fragments (Ryan 2015a) and sheets and foam may therefore be excreted when arriving on their breeding locations on the continent.

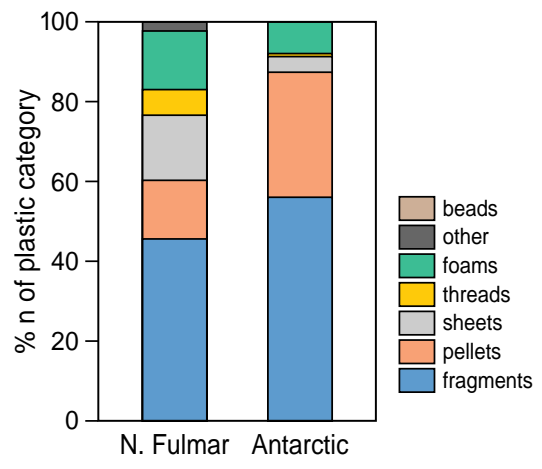

Figure 7.6. Number of plastics in northern fulmars and procellariform seabirds from Antarctica per plastic category. Graph is based on data shown in Online Supplement Table 3.2b (N. fulmars) and 3.5a. 
Foam in northern fulmar samples was identified as mainly being PVC (59\% in terms of number, $42 \%$ of mass). PS comprised of only $5 \%$ in numbers and $2 \%$ of mass of foams found in the fulmar stomachs. A possible explanation might be the extremely low specific mass of PS foams, which may lead to rapid disappearance from open ocean environments where fulmars forage. Different rates of processing on the materials in the bird's stomachs are another speculative interpretation. PVC was the predominant polymer type ingested by little auks (Alle alle) from the Arctic (Amélineau et al. 2016), however, almost all plastics (97.2\%) the authors found were microfibres, a plastic category not considered in our study. PVC is a plastic type, usually containing high quantities of phthalate plasticizers (Hermabessiere et al. 2017). These substances are known for leaching from the plastics and its endocrine disruptive characteristics (Oehlmann et al. 2009). PVC is the third most common plastic type in our seabird sample and the associated risks of ingesting PVC should be of concern (Rochman 2015).

In seabirds, no polymer type specific preference has been reported. Plastic uptake by seabirds might simply reflect the background availability of plastics produced through the time and the distribution of marine plastic debris on the ocean surface. PE and PP are less dense than seawater and almost all polymers, including those heavier than seawater, can be found in their expanded form as foam. This might explain the great availability of these polymers for the uptake by surface seizing seabirds. From the beginning of the industrial plastic production in the 1950s and onwards, PE and PP have always been the most commonly produced polymers over time (Geyer et al. 2017), resulting in widespread disposal.

\section{Conclusion}

FTIR has become a common identification method in plastic research. NIR has been used to a less extent, however, both methods are suitable for plastic ingestion studies. Caution should be given to the fact, that especially FTIR tends to misidentify natural hard prey items as being plastic. Therefore, basic knowledge of natural diet of the organism studied is necessary. Our results of plastic ingested by seabirds indicate that the plastic category available at a specific location or in a specific time frame seems to be the driving factor of polymer proportions in seabirds, rather than regional, global or temporal circumstances.

\section{Acknowledgements}

Susanne Kühn is funded through the Joint Program Initiative (JPI) Oceans PLASTOX (Direct and indirect ecotoxicological impacts of microplastics on marine organisms) project by the Netherlands Organisation for Scientific Research (NWO) under the project number ALW-NWO 856.15.001. Arend Bolt of the Van Gansewinkel Group kindly helped us in polymer identification by lending us the NIR Phazir device for this project and a number of known reference materials. Marion Egelkraut-Holtus (Shimadzu Europa GmbH) has been of great help in FTIR technology. 
$x^{2} \times x^{-3}$

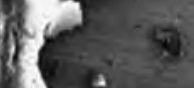<smiles>C1CCC2(CC1)CCCC2</smiles>
11

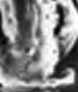

$f_{1}^{2}$

10

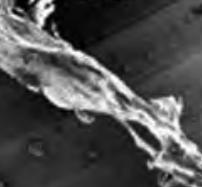

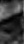

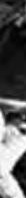

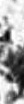


$\operatorname{lig}$

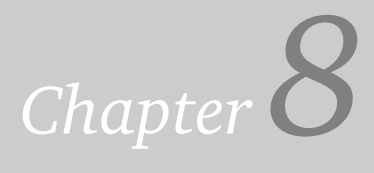

3

\section{Marine microplastic: Preparation of} relevant test materials for laboratory assessment of ecosystem impacts

Susanne Kühn, Albert van Oyen, Andy M. Booth, André Meijboom, Jan A. van Franeker

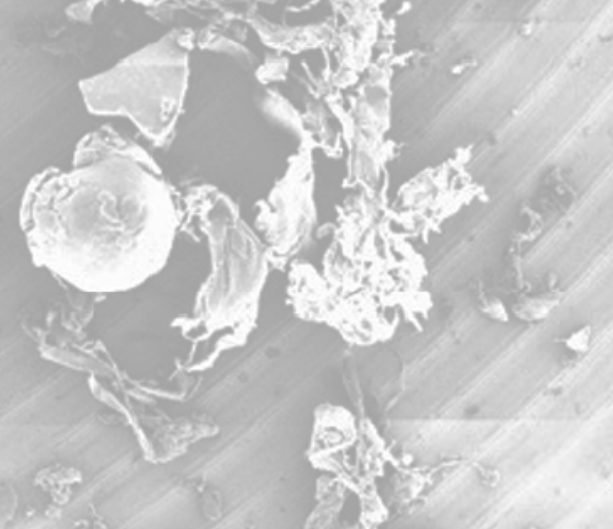




\begin{abstract}
Studies investigating the effects of plastic litter on marine biota have almost exclusively utilised pristine plastic materials that are homogeneous in polymer type, size, shape and chemical composition. This is particularly the case for microplastics $(<5 \mathrm{~mm})$, where collecting sufficient quantities from the marine environment for use in laboratory impacts studies is simply not feasible. Weathered plastics collected from the marine environment show considerable physical and chemical differences to pristine and post-production consumer plastics. For this study, macroplastic litter was collected on a Dutch beach and cryo-milled to create a microplastic mixture for environmental impact assessments. The sample composition followed proportions of marine plastic litter types observed in an earlier large beach clean-up. Polymer composition of the sample was assessed by infrared spectroscopy (ATR-FTIR) and differential scanning calorimetry analysis (DSC). The particle size distribution of the cryo-milled microplastics showed that particles $0.5-2.0 \mathrm{~mm}$ represented $68 \%$ of mass, but smaller sizes $(<2 \mathrm{~mm})$ strongly dominated numerically. Inductively coupled plasma spectroscopy (ICP-MS and ICP-OES) analysis of the microplastic mixture revealed a broad range of metals and other elements (e.g. $\mathrm{Al}, \mathrm{Cd}, \mathrm{Cr}, \mathrm{Fe}, \mathrm{Mg}, \mathrm{Pb}, \mathrm{S}$ and $\mathrm{Zn}$ ), representing common inorganic additives used as colorants, fillers and stabilisers. GC-MS analysis identified a broad range of organic plasticisers, stabilisers, antioxidants and flame retardants. Comparison of different analytical approaches showed that creation of a homogeneous microplastic mixture is possible, representing a first step in closing the gap between laboratory studies with pristine materials and realistic scenarios with weathered microplastic.
\end{abstract}




\section{Introduction}

Widely acknowledged as a global phenomenon, marine litter is known to negatively affect the environment in several ways. In addition to the economic costs of clean-ups and potential danger of interference with ships (Mouat et al. 2010), the potential hazard to marine organisms has raised concerns and led to marine litter being identified as an emerging issue by the United Nations Environmental Program (UNEP 2011). Although impacts such as entanglement or the ingestion of marine litter have been recorded for thousands of individuals representing many species (Kühn et al. 2015; Gall \& Thompson 2015), linking negative changes in a population to marine debris has been proven to be a difficult task (Browne et al. 2015).

Evaluating the potential impacts of exposure to marine litter in the natural environment is nearly impossible, and therefore laboratory-based studies are necessary to generate such knowledge. However, reported studies investigating the possible effects of plastic litter on marine biota have almost exclusively utilised pristine plastic materials that are homogeneous in polymer type, size, shape and chemical composition. This is particularly the case for microplastics (marine litter $<5 \mathrm{~mm}$; Arthur et al. 2009), as collecting sufficient quantities from the marine environment for use in laboratory impacts assessment is simply not feasible. As a result, most of the currently available data does not reflect environmentally realistic exposure scenarios (Phuong et al. 2016), and there is an urgent need to conduct studies with more relevant plastic test materials from a wide range of consumer products weathered by exposure to the conditions in the marine environment (Jahnke et al. 2017).

Once released into the marine environment, plastic litter immediately begins to undergo a variety of weathering processes that can significantly change the physical and chemical properties of the pristine material (Fotopoulou \& Karapanagioti 2012; Gewert et al. 2015). It is these very processes that are known to contribute to the formation of microand nanoplastic particles. The type and degree of weathering is highly dependent upon the marine environmental compartment the plastic enters and the length of time it has been in the marine environment. The process is complex and influenced by polymer type and the presence of additive chemicals that are added to individual raw plastic materials to create a product with specific physical and chemical properties (e.g. flexibility, colour, function and protection against degradation processes). These additives include, but are not restricted to, plasticizers, flame retardants, colorants, lubricants, antioxidants and UV stabilizers.

Crucially, weathered plastics collected from the marine environment show considerable physical and chemical differences to pristine and post-production consumer plastics (Jahnke et al. 2017). For example, exposure of plastic litter to UV light decreases the stability of polymers leading to fragmentation and changes in chemical composition (Singh \& Sharma 2008), may enhance adsorption of hazardous substances to the plastic from surrounding seawater (Teuten et al. 2009; Mato et al. 2001), and promote release of additive chemicals (Artham \& Doble 2009). Potential endocrine disruption as well as 
mutagenic and toxic effects of a variety of additive chemicals has been reported by several previous studies (reviewed and summarized recently by Hermabessiere et al. 2017). However, detecting the effects of these substances on wildlife has been difficult (Werner et al. 2016). Experimental effect studies that use pristine materials without additive chemicals may well underestimate the overall impacts of plastic litter exposure to marine organisms.

In the framework of the Joint Programme Initiative on Oceans (JPI Oceans) project PLASTOX, the preparation of a more environmentally relevant sample of microplastic was considered important for allowing more relevant impact assessments. To achieve an environmentally realistic sample of marine plastic litter, the proportions of different types of plastic litter were derived from those documented in an earlier large-scale beach clean-up on the island of Texel, The Netherlands (Van Franeker 2005). The generated microplastic reference materials provide more insight on the diversity of beached plastics on beaches and at the same time the sample can be used for future experimental work on the effects of marine plastic debris ingestion on wildlife. Given the complexity of the final microplastic mixture and the potential presence of a broad range of organic and inorganic additives, a battery of analytical techniques has been applied to fully characterize the physicochemical properties of the final reference material.

\section{Methods}

\section{Background data}

The composition of the marine litter-derived (MLD) microplastic mixture produced is based on a beach clean-up of the beach of the island of Texel (Van Franeker 2005; summarized in English in: Van Franeker \& Meijboom 2006), the westernmost Wadden Sea island of the Netherlands $\left(53^{\circ} 1^{\prime} \mathrm{N}, 4^{\circ} 8^{\prime} \mathrm{E}\right)$. The western coast of Texel has a $30 \mathrm{~km}$ sandy beach facing the North Sea, with predominantly western winds occurring all year round. Texel was selected as plastics collected during the 2005 beach clean-up were extensively quantified in terms of number of items and mass. During the 2005 study, approximately 15 tonnes of plastic debris were collected, with an average of 508 plastic litter items per kilometre. The plastic litter collected was weighed in two main categories: threadlike materials (ropes/nets) as compared to all other plastic consumer debris. The ropes and nets category dominated with an average mass of $229 \mathrm{~kg} / \mathrm{km}$, all types of other plastic debris had a combined average mass of $169 \mathrm{~kg} / \mathrm{km}$. In counts of other plastic debris, a range of further categories and subcategories of plastic consumer wastes was specified. By weighing a range of individual items belonging to the various sub-categories, their known numerical abundance could be used to estimate the weight of each (sub)category per $\mathrm{km}$ of beach (Table 8.1). Some of the subcategories from the 2005 collection appear to have high estimated average mass values, but these result from the occurrence of a very small number of large and heavy items which skew the contribution from the much larger number of small objects in each subcategory. For example, foam average mass was high 
because of e.g. some big matrasses, the average for sheet-like materials was increased by large transport- or agricultural sheets, and in the other 'miscellaneous' subcategory, average mass was elevated by infrequent odd but large items such as building materials, car parts, big baskets or buoys.

\section{Plastic litter collection}

In April and August 2016, plastic litter was collected from the same beaches on Texel, with the goal of obtaining source materials matching the composition of the plastic litter collected in the 2005 study. These items were randomly collected over all the beach areas, including the tidal zone and older strandlines. Items of uncertain provenance (e.g. beach toys and drink bottles that may have been brought recently to the beach) were only collected when there was clear evidence of degradation or when the occurrence of biofouling indicated they had been in seawater for a considerable time. Items heavily coated with sand were carefully rinsed with fresh water, otherwise they were simply dried at $18-20^{\circ} \mathrm{C}$ for several weeks before residual sand was gently removed with a hand brush to minimize potential changes to the surface.

Table 8.1. The average number of items per kilometre, the average item mass and estimated total mass of each beach plastic litter category and sub-category as collected during the 2005 beach clean-up on Texel, The Netherlands.

\begin{tabular}{|c|c|c|c|c|c|}
\hline \multicolumn{2}{|c|}{ Category } & \multirow{2}{*}{$\begin{array}{l}\text { Sub-category } \\
1.1 \text { Foams }\end{array}$} & \multirow{2}{*}{$\begin{array}{r}\begin{array}{c}\text { Average } \\
\mathrm{n} / \mathrm{km}\end{array} \\
28.1\end{array}$} & \multirow{2}{*}{$\begin{array}{c}\begin{array}{c}\text { Estimated } \\
\text { item mass }(\mathrm{g})\end{array} \\
712\end{array}$} & \multirow{2}{*}{$\begin{array}{c}\begin{array}{c}\text { Estimated } \\
\text { mass per km }(\mathrm{g})\end{array} \\
20013\end{array}$} \\
\hline 1 & Foam & & & & \\
\hline \multirow[t]{2}{*}{2} & Plastic bottles & 2.1 Drink bottles & 69.9 & 23 & 1583 \\
\hline & & 2.2 Other bottles & 34.6 & 32 & 1109 \\
\hline \multirow[t]{2}{*}{3} & Fish boxes, jerrycans & 3.1 Fish boxes & 2.3 & 2236 & 5165 \\
\hline & & 3.2 Jerrycans & 17.2 & 642 & 11067 \\
\hline \multirow[t]{2}{*}{4} & Other packaging & 4.1 Sheets (bags \& industrial) & 55.0 & 888 & 48834 \\
\hline & & 4.2 Sixpack, strapbands & 9.3 & 3 & 25 \\
\hline 5 & Gloves & 5.1 Working gloves & 4.2 & 99 & 419 \\
\hline \multirow[t]{5}{*}{6} & Other plastic materials & 6.1 Balloons & 6.0 & 5 & 31 \\
\hline & & 6.2 Other fishery equipment & 1.7 & 28 & 48 \\
\hline & & 6.3 Silicone/grease tubes & 2.6 & 32 & 81 \\
\hline & & 6.4 Food packaging & 42.2 & 12 & 527 \\
\hline & & 6.5 Miscellaneous & 130.4 & 614 & 80098 \\
\hline \multirow[t]{4}{*}{7} & Threadlike & 7.1 Ropes and nets & 103.9 & 2204 & 229000 \\
\hline & & Totals & 507.5 & & 398000 \\
\hline & & plastic consumer debris & 404 & & 169000 \\
\hline & & nets and ropes & 104 & & 229000 \\
\hline
\end{tabular}




\section{Sample preparation}

MIXTURE COMPONENTS

Aiming for the broadest possible diversity of plastic objects in the MLD microplastic mixture, a wide range of different items for all subcategories of plastic was collected from the beach on Texel in 2016. As much as possible different items were used to compose each subcategory of the mixture. This meant that in some of the subcategories only small parts of individual items were used. Where possible equal mass from each object were taken.

\section{CRYOGENIC MILLING}

An ultra-centrifugal stainless steel mill (Retsch ZM 200 with a combined cyclone) was used to grind the raw material. To avoid overheating of the sample and possible changes to the polymer composition, liquid nitrogen $\left(-196^{\circ} \mathrm{C}\right)$ was added for 30 minutes to increase brittleness and suitability for milling. The particle size specification of the milling chamber was $3 \mathrm{~mm}$. Pre-tests revealed macroplastic mixtures containing all the different materials did not mill successfully. Therefore, the final milling process was applied to individual sub-samples of nets \& ropes (7), foam (1), sheets (4.1) and rigid plastics (2, 3, 4.1, $5,6)$, which were subsequently recombined to the original composition. The samples were stored in HDPE Kautex bottles.

\section{Physical characterisation}

The milling process generated a MLD microplastic mixture with a very broad size distribution. To facilitate the process of assessing the particle size distribution of the sample, the bulk microplastic mixture was first sorted by size using a sieve tower comprising sieves of six different size classes: 4.5, 3.15, 2.0, 1.0, 0.5 and $0.2 \mathrm{~mm}$ (Retsch AS200 Basic). Determination of the particle size distribution (PSD) of the un-sieved MLD microplastic mixture was attempted using a laser diffraction. However, no reliable results could be achieved due to the irregular shape of the particles, the low density of foam, and aggregation at the media surface.

Two sub-samples of each fraction of the MLD microplastic mixture were manually counted, the results combined, and an average PSD determined. Sub-samples from the larger size fractions $(>4.5,3.15,2.0$ and $1.0 \mathrm{~mm}$ ) were counted under a light microscope. In addition to counting the total number of particles, they were categorized into fragments, foam, sheets, threads and dust (small fibres that often aggregated to bigger 'dust balls') to see possible variations per size class in relation to the original composition of the sample (For pictures see Online Supplement 3, Figure 3.2.). For the smaller size fractions ( 0.5 and $<0.2 \mathrm{~mm}$ ), sub-samples were placed into a Bogorov counting chamber with $70 \%$ ethanol (to reduce aggregation). Pictures of each Bogorov compartment were taken with a camera mounted on a microscope (AxioCam MRc with AxioVision 40 V 4.8.2.0 software; Zeiss, Germany), and the number of particles recorded (see pictures in Online Supplement 3, Figure 3.3.).

To visualise the different particle types and physical properties, the microplastic mixture was also subjected to scanning electron microscopy (SEM). SEM imaging was performed 
using a Hitachi S-3400N electron microscope equipped with an Oxford Instruments Aztec EDS system. Samples were dispersed onto an aluminium sample holder and lightly sputter-coated with gold to improve conductivity and stability. To image a representative portion of the sample, a montage of 48 images was created using the Aztec software.

\section{Chemical characterisation}

A comprehensive chemical profiling was conducted of the MLD microplastic mixture to determine the polymer composition and the organic chemical and metal profile and concentrations associated with the particles (either as additives or pollutants adsorbed to the surface). To determine the polymer distribution of the macroplastic litter items collected from the beach, each item was analysed by attenuated total reflection Fourier transform infrared spectroscopy (ATR-FTIR; Shimadzu Prestige 21, 10 Scans, 4 libraries with 325 spectra) and differential scanning calorimetry (DSC; Mettler STARe System DSC3 Basic). While FTIR-ATR was used to describe the plastic composition of each of the individual samples, DSC was only used to analyse the homogenized sample mixture. Due to the low concentration of LDPE and sensitivity of the instrumentation used, no distinction has been made between HDPE and LDPE.

DSC analysis of MLD microplastic mixture was used to verify that the same polymer composition as the original macroplastic mixture had been achieved. DSC identifies polymer types through detection of either endothermic or exothermic reactions. The temperature of crystallisation (CP) is measured during the cooling processes. During the first run, samples were heated to $200{ }^{\circ} \mathrm{C}$ and cooled again. In a second run, samples were heated to $300^{\circ} \mathrm{C}$ at $10^{\circ} \mathrm{C} \mathrm{min}{ }^{-1}$. As DSC measurements on polymers are strongly influenced by the thermal history and the morphology of the sample, it is important to have a previous heating cycle completed while the measurements are carried out at the second heating stage. In the current study, three sub-samples of the MLD microplastic mixture were analysed using DSC so that average values could be determined.

The metals associated with the MLD microplastic mixture were determined using inductively coupled plasma optical emission spectroscopy (ICP-OES), inductively coupled plasma mass spectrometry (ICP-MS) and energy dispersive x-ray spectrometry (EDX). The three different methods were evaluated and compared to identify which represents the most accurate and efficient option for future routine analysis of metals in plastics. For ICPOES analysis, $10 \mathrm{~mL}$ of nitric acid $\left(\mathrm{HNO}_{3} 65 \%\right.$; Merck) was added to sub-samples (3 $\mathrm{g}$ each; triplicate) of the MLD microplastic mixture. After $30 \mathrm{~min}$, the samples were placed in a microwave oven (CEM Mars 5 Digestion) at 800 watt and heated to $230^{\circ} \mathrm{C}$. The digests were diluted in deionised water $(50 \mathrm{~mL})$ prior to triplicate analysis for 34 elements (Shimadzu ICPE-9000 fitted with a 10UES Nebulizer and a plasma Mini Torch).

For ICP-MS analysis, triplicate MLD microplastic mixture samples were diluted in $\mathrm{HNO}_{3}$ and hydrogen fluoride (HF) and heated at $220^{\circ} \mathrm{C}$ for 20 minutes in a microwave (Milestone Ethos). The digested samples were analysed in triplicate using an Agilent 8800 Triple Quadrupole ICP-MS fitted with a SPS 4 Autosampler. ${ }^{89} \mathrm{Y}$ and ${ }^{185}$ Re were used as internal standards and quantified against standards from Inorganic Ventures. A smaller 
number of target elements were screened using this method (13 different elements), as the main goal was to investigate the sensitivity of the method compared to ICP-OES. To provide an alternative, non-destructive method to ICP-based analyses, the MLD microplastic mixture was also subjected to EDX analysis (Shimadzu EDX-720). Internal calibration using standard reference materials and blank measurements were performed daily before each use. In principle, all elements heavier than carbon can be measured using EDX but is most effective for elements heavier than magnesium. In contrast to the two ICP-based methods, EDX is also able to measure halogens such a chlorine and bromine. In the current study, calibration and quantification was only possible for seven elements using EDX owing to the high costs involved with calibrating individual elements. Four elements (Ba, Cd, $\mathrm{Cr}$ and $\mathrm{Pb}$ ) were quantified by all three methods. They were statistically compared to see whether there are significant differences between the three methods for each of the elements. An ANOVA followed by a Tukey test was performed using R (R version 3.3.1 (2016-06-21); R Core Team 2014).

The presence of organic chemical additives and pollutants was investigated using gas chromatography-mass spectrometry (GC-MS). Analysis comprised single samples without any replicates. Organic chemicals were extracted by adding $1 \mathrm{~mL}$ of ethyl acetate solvent to $100 \mathrm{mg}$ of the MLD microplastic mixture and ultrasonicating for $10 \mathrm{~min}$ (Llompart et al. 2013). The sample was then centrifuged (4000 rpm) to settle any remaining particles

Table 8.2. Target mass and percentage contribution of different subcategories in the MLD microplastic mixture as derived from mass per km in Table 8.1, with the number of different beach litter items used to compose the samples.

\begin{tabular}{|c|c|c|c|c|}
\hline Category & \multirow{2}{*}{$\begin{array}{l}\text { Sub-category } \\
1.1 \text { Foams }\end{array}$} & \multirow{2}{*}{$\begin{array}{c}\begin{array}{c}\text { Target mass } \\
(\mathrm{g} / \mathrm{kg})\end{array} \\
50\end{array}$} & \multirow{2}{*}{$\begin{array}{c}\% \text { of mass } \\
5.0 \%\end{array}$} & \multirow{2}{*}{$\begin{array}{c}\begin{array}{c}\mathrm{N} \text { items used to } \\
\text { produce microplastic } \\
\text { mixture }\end{array} \\
29\end{array}$} \\
\hline 1 Foam & & & & \\
\hline 2 Plastic bottles & 2.1 Drink bottles & 4 & $0.4 \%$ & 5 \\
\hline & 2.2 Other bottles & 3 & $0.3 \%$ & 12 \\
\hline 3 Fish boxes, jerrycans & 3.1 Fish boxes & 13 & $1.3 \%$ & 4 \\
\hline & 3.2 Jerrycans & 28 & $2.8 \%$ & 13 \\
\hline 4 Other packaging & 4.1 Sheets (bags \& industrial) & 123 & $12.3 \%$ & 67 \\
\hline & 4.2 Sixpack, strapbands & 0.1 & $0.0 \%$ & 12 \\
\hline 5 Gloves & 5.1 Working gloves & 1.1 & $0.1 \%$ & 3 \\
\hline 6 Other plastic materials & 6.1 Balloons & 0.1 & $0.0 \%$ & 3 \\
\hline & 6.2 Other fishery equipment & 0.1 & $0.0 \%$ & 7 \\
\hline & 6.3 Silicone/grease tubes & 0.2 & $0.0 \%$ & 2 \\
\hline & 6.4 Food packaging & 1.3 & $0.1 \%$ & 33 \\
\hline & 6.5 Miscellaneous & 201.3 & $20.1 \%$ & 52 \\
\hline 7 Threadlike & 7.1 Ropes and nets & 575.4 & $57.5 \%$ & 109 \\
\hline & Total & 1000 & $100.0 \%$ & 351 \\
\hline
\end{tabular}


before the supernatant was removed for analysis. Non-target screening was performed on an Agilent GC-MS-5977 instrument. A $1 \mu \mathrm{L}$ aliquot was injected on a 15m HP-5MS column with an inner diameter of $250 \mu \mathrm{m}$ and a film thickness of $0.25 \mu \mathrm{m}$. Injection was performed in the splitless mode at a detector temperature of $250^{\circ} \mathrm{C}$. The column temperature program was: $60^{\circ} \mathrm{C}$ for 4 minutes, then ramped to $250^{\circ} \mathrm{C}$ at a rate of $15^{\circ} \mathrm{C} /$ minute, hold time 4 minutes, and a second ramping up to $315^{\circ} \mathrm{C}$ at $15^{\circ} \mathrm{C} / \mathrm{minute}$. The mass spectrometer was operated in the EI mode with a scan range from m/z 30 to m/z 800 . Identification of organic chemicals was based on NIST library matching of mass spectra.

\section{Results}

\section{Microplastic mixture preparation}

In total, 351 items of plastic litter were used for preparation of the microplastic mixture. The target mass required for each sub-category, the number of items collected within each sub-category, and the mass of individual litter items required to produce $1 \mathrm{~kg}$ of the target MLD microplastic mixture composition is presented in Table 8.2. Pictures and the polymer type of all individual items are presented in the Online Supplements 1, 4 and 5.

A mass decrease was observed following cryo-milling, possibly due to the removal of sand during the sample preparation, where items had to be cut into smaller pieces to fit into the cryo-mill (details of the rate of return are shown in the Online Supplement 2, Table 2.1.). To determine the recovery efficiency, the mass of the individual milled subcategories was compared to the mass of macroplastic used at the start of the process. As differences in recovery efficiency affect the composition of the MLD microplastic mixture, the material generated from milling the individual sub-categories was homogenized through combining the three subcategories by mass to achieve the target mass composition.

\section{Physical characterisation}

The mass contribution of each size fraction to the total microplastic mixture is shown in Figure 8.1. Most of the mass of the MLD microplastic mixture is in the size range 0.5-2.0 $\mathrm{mm}$, accounting for $\sim 68 \%$ of the total mass. The $0.2-0.5 \mathrm{~mm}$ size fraction accounts for $\sim 18 \%$, while particles $<0.2 \mathrm{~mm}$ account for $\sim 9 \%$. No significant amount of material was present in the $3.15-4.5 \mathrm{~mm}$ and $>4.5 \mathrm{~mm}$ fractions. The total particle number in each size fraction was determined by manual counting (Table 8.3). Using the total number of particles counted in each fraction, a simplified PSD was produced (Figure 8.1, Online Supplement 3). The number of particles belonging to the different sub-categories varies across the different size fractions generated, but 1 gram of the complete MLD microplastic mixture contains an estimated 410000 particles.

SEM imaging of the microplastic mixture revealed a broad range of particle shapes and morphologies (Figure 8.2), confirming that most particles in the MLD microplastic mixture are highly irregular in nature. 


\section{Chemical characterisation}

All individual plastic litter items $(n=351$, Table 8.3) used in the preparation of the MLD microplastic mixture were analysed using FTIR-ATR prior to milling. In terms of mass contribution, the main polymers are polyethylene (PE) and polypropylene (PP), which together represent $89 \%$ of the marine litter (Figure 8.3, Online Supplement 5, Table 5.1.). Much smaller contributions to the plastic litter come from a range of other polymer types

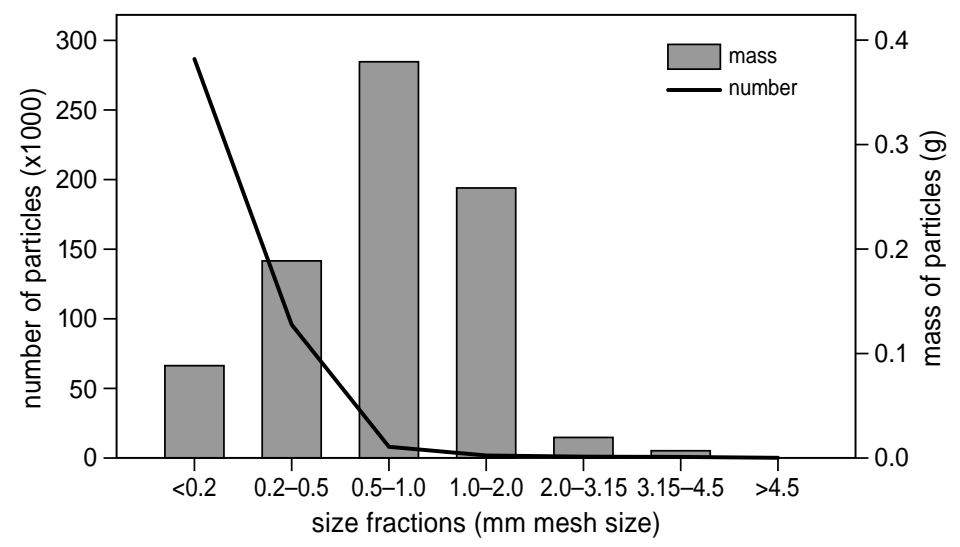

Figure 8.1. Particle size distribution of 1 gram of the bulk MLD microplastic mixture (line; left y-axis) is heavily skewed to the smallest particle sizes, where the mass contribution of the individual size fractions reflects an approximately normal distribution around the $0.5-1.0 \mathrm{~mm}$ fraction (bars; right y-axis).

Table 8.3. Number of particles present in each size fraction of the MLD microplastic mixture (subsamples counted: 2; standard deviation given in parentheses). Larger size fractions are counted by sub-category, but this was not possible for the smaller size fractions (n/a).

\begin{tabular}{|c|c|c|c|c|c|c|c|}
\hline \multirow{2}{*}{$\begin{array}{l}\text { Size fraction } \\
(\mathrm{mm})\end{array}$} & \multicolumn{5}{|c|}{ No. of particles per class } & \multirow{2}{*}{$\begin{array}{l}\text { Total no. } \\
\text { particles }\left(\mathrm{g}^{-1}\right)\end{array}$} & \multirow{2}{*}{$\begin{array}{l}\% \text { no. of } \\
\text { particles }\end{array}$} \\
\hline & Sheets & Threads & Foam & Fragments & Dust & & \\
\hline$>4.5$ & 0.0 & 0.0 & 0.0 & 0.0 & $\begin{array}{c}3.6 \\
( \pm 5.1)\end{array}$ & $\begin{array}{c}3.6 \\
( \pm 5.1)\end{array}$ & 0.000 \\
\hline $3.15-4.5$ & $\begin{array}{c}114.2 \\
( \pm 55.5)\end{array}$ & 0.0 & $\begin{array}{c}1088.5 \\
( \pm 160.5)\end{array}$ & $\begin{array}{c}6.4 \\
( \pm 9.0)\end{array}$ & $\begin{array}{c}56.7 \\
( \pm 25.9)\end{array}$ & $\begin{array}{c}1265.8 \\
( \pm 199.2)\end{array}$ & 0.003 \\
\hline $2.0-3.15$ & $\begin{array}{c}190.8 \\
( \pm 37.7)\end{array}$ & $\begin{array}{c}27.7 \\
( \pm 12.7)\end{array}$ & $\begin{array}{c}1069.0 \\
( \pm 42.9)\end{array}$ & $\begin{array}{c}127.6 \\
( \pm 21.4)\end{array}$ & $\begin{array}{c}9.6 \\
( \pm 12.9)\end{array}$ & $\begin{array}{c}1424.5 \\
( \pm 16.4)\end{array}$ & 0.007 \\
\hline $1.0-2.0$ & $\begin{array}{l}1133.1 \\
( \pm 9.2)\end{array}$ & $\begin{array}{c}365.7 \\
( \pm 29.9)\end{array}$ & $\begin{array}{c}558.8 \\
( \pm 73.6)\end{array}$ & $\begin{array}{c}737.9 \\
( \pm 89.0)\end{array}$ & $\begin{array}{c}6.1 \\
( \pm 5.8)\end{array}$ & $\begin{array}{c}2801.5 \\
( \pm 189.1)\end{array}$ & 0.187 \\
\hline $0.5-1.0$ & $\mathrm{n} / \mathrm{a}$ & $\mathrm{n} / \mathrm{a}$ & $\mathrm{n} / \mathrm{a}$ & $\mathrm{n} / \mathrm{a}$ & $\mathrm{n} / \mathrm{a}$ & $\begin{array}{c}\mathbf{1 8 8 0 0 . 0} \\
( \pm 212.1)\end{array}$ & 1.833 \\
\hline $0.2-0.5$ & $\mathrm{n} / \mathrm{a}$ & $\mathrm{n} / \mathrm{a}$ & $\mathrm{n} / \mathrm{a}$ & $\mathrm{n} / \mathrm{a}$ & $\mathrm{n} / \mathrm{a}$ & $\begin{array}{c}\mathbf{5 0 6 7 5 0 . 0} \\
( \pm 32173.4)\end{array}$ & 24.702 \\
\hline \multirow[t]{2}{*}{$<0.2$} & $\mathrm{n} / \mathrm{a}$ & $\mathrm{n} / \mathrm{a}$ & $\mathrm{n} / \mathrm{a}$ & $\mathrm{n} / \mathrm{a}$ & $\mathrm{n} / \mathrm{a}$ & $\begin{array}{c}3173100.0 \\
( \pm 290196.6)\end{array}$ & 73.268 \\
\hline & & & & & Total & 411587 & 100 \\
\hline
\end{tabular}


( $<4 \%$ each), with approximately $3 \%$ remaining unidentifiable. Semi-quantitative DSC analysis of the MLD microplastic mixture confirmed these results, with PE, PP and polyamide (PA) being the most abundant polymers. However, an accurate quantification of each polymer is not possible with this method as different polymers in a complex mixture will interact during the melting process, changing or eliminating the crystallisation and melting temperatures.

Results of the elemental analysis of the MLD microplastic mixture, conducted by the three different approaches, are presented in Table 8.4. The most extensive screening of elements present in the MLD microplastic mixture was conducting using ICP-OES. Data showed that 21 of the 34 elements screened were present at concentrations above the instrumental limits of detection. Concentrations of the detectable elements ranged from $4639.2 \mu \mathrm{g} / \mathrm{g}( \pm 1665.9 \mu \mathrm{g} / \mathrm{g})$ for calcium to $1.2 \mu \mathrm{g} / \mathrm{g}( \pm 0.1 \mu \mathrm{g} / \mathrm{g})$ in the case of gallium. In total, 13 heavy metals were detected ( $\mathrm{Cd}, \mathrm{Cr}, \mathrm{Cu}, \mathrm{Fe}, \mathrm{Ga}, \mathrm{In}, \mathrm{Mn}, \mathrm{Mo}, \mathrm{Ni}, \mathrm{Pb}, \mathrm{Pd}, \mathrm{Sn}$, $\mathrm{Zn})$, with their concentrations ranging from $1591.2 \mu \mathrm{g} / \mathrm{g}( \pm 163.2 \mu \mathrm{g} / \mathrm{g})$ for iron to 1.2

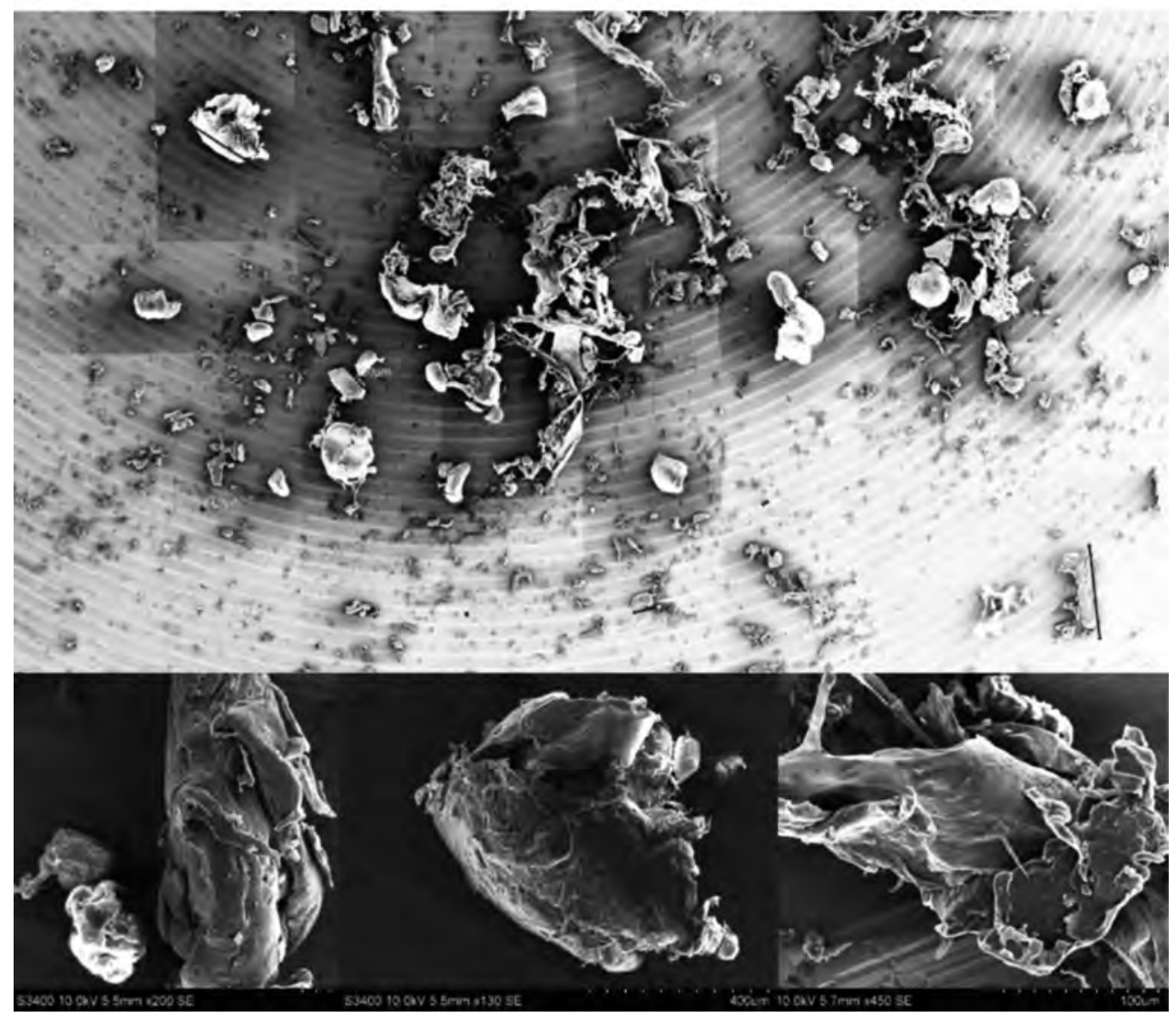

Figure 8.2. Scanning electron microscope images of the cryogenically milled MLD microplastic mixture. Top: a composition of 48 pictures. Bottom: detailed images of selected individual particles. 


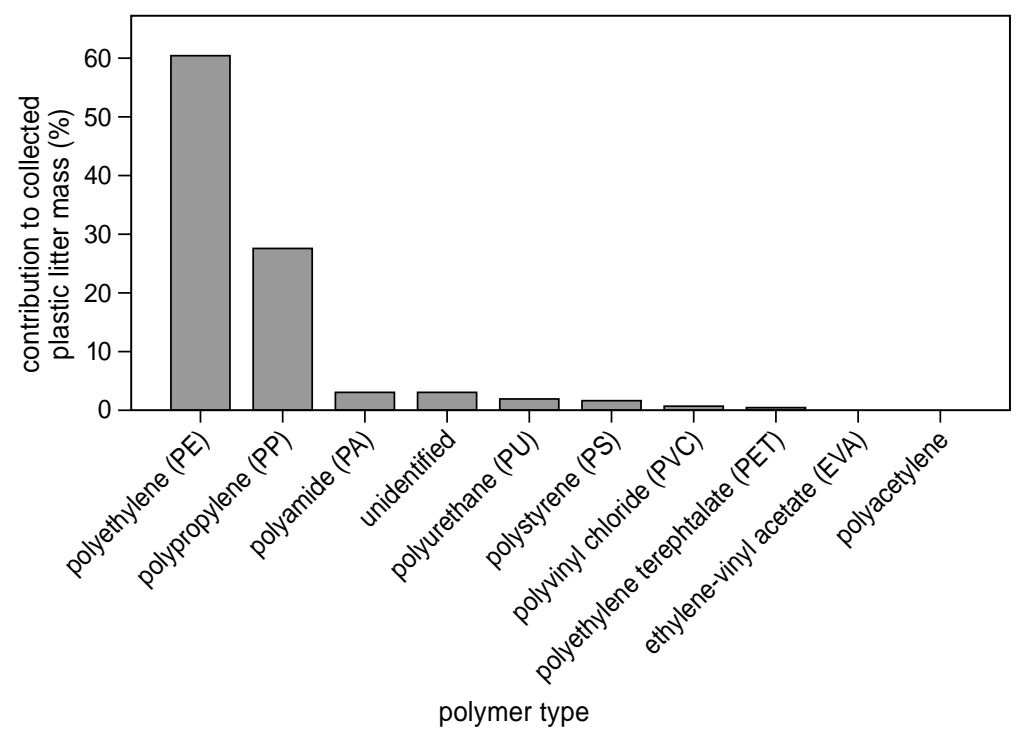

Figure 8.3. Percentage contribution by mass of different polymer types to the collected plastic litter (based on $1 \mathrm{~kg}$ and 351 individual items). Polymer type was identified using ATR-FTIR analysis.

$\mu \mathrm{g} / \mathrm{g}( \pm 0.1 \mu \mathrm{g} / \mathrm{g})$ for gallium. The heavy metals $\mathrm{Ag}, \mathrm{Bi}, \mathrm{Co}, \mathrm{Hg}, \mathrm{Rh}$, and $\mathrm{V}$ were not present at detectable concentrations. For light metals (Al, $\mathrm{Ba}, \mathrm{Ca}, \mathrm{K}, \mathrm{Mg}, \mathrm{Na}, \mathrm{Sr}, \mathrm{Ti}$ ), the concentration ranged from $4639.2 \mu \mathrm{g} / \mathrm{g}( \pm 1665.9 \mu \mathrm{g} / \mathrm{g})$ for calcium to $28.0 \mu \mathrm{g} / \mathrm{g}( \pm 6.8$ $\mu \mathrm{g} / \mathrm{g}$ ) for strontium. No metalloid elements (As, B, Sb, Se) were present at detectable concentrations. A range of 13 heavy and light metal elements (Al, Ba, Cd, Co, Cr, $\mathrm{Cu}, \mathrm{Fe}$, $\mathrm{Mn}, \mathrm{Mo}, \mathrm{Ni}, \mathrm{Pb}, \mathrm{V}$ and $\mathrm{Zn}$ ) were also determined using a combination of $\mathrm{HF}$ digestion and ICP-MS analysis (Table 8.4). Finally, a small number of elements (Ba, $\mathrm{Br}, \mathrm{Cd}, \mathrm{Cr}, \mathrm{Pb}, \mathrm{Sb}$, Se) were quantified using EDX.

In total, 13 common elements ( $\mathrm{Al}, \mathrm{Ba}, \mathrm{Cd}, \mathrm{Co}, \mathrm{Cr}, \mathrm{Cu}, \mathrm{Fe}, \mathrm{Mn}, \mathrm{Mo}, \mathrm{Ni}, \mathrm{Pb}, \mathrm{V}$ and $\mathrm{Zn}$ ) were studied using both ICP-OES and ICP-MS instrumentation. The concentrations determined are similar with both methods, although concentrations are always higher with ICPMS than ICP-OES. Furthermore, elements identified at very low concentrations by ICP-MS (e.g. Co and V; 3.0 and $3.2 \mu \mathrm{g} / \mathrm{g}$, respectively), were below the detection limits of ICPOES. Additionally, 4 elements quantified by ICP-based analysis (Ba, $\mathrm{Cd}, \mathrm{Cr}, \mathrm{Pb}$ ) were also quantified in the microplastic mixture using EDX. Comparison of the concentrations determined by the three different techniques is presented in Figure 8.4. Generally, EDX analysis was able to provide elemental concentration data comparable to that of ICP-based techniques, only $\mathrm{Cr}$ showed a significant difference between all the three methods respectively $(\mathrm{p}<0.05)$ GC-MS screening analysis of the MLD microplastic mixture identified the presence of $>50$ different organic compounds. Of these, 22 were identified with a $>80 \%$ confidence match by the NIST mass spectral library and are summarised in Table 8.5. A detailed online and literature search of these chemicals indicated most were common 
Table 8.4. Elemental profile of the MLD microplastic mixture conducted by ICP-OES $(n=6)$, ICP-MS $(n=3)$ and $\operatorname{EDX}(n=6)$, with standard deviation presented in parentheses. The abbreviation n.d. stands for 'not detected', whilst '-' corresponds to elements that were not measured using a specific technique. ' $\mathrm{P}$ ' is used to denote EDX measurements where an element was detectable but not quantifiable.

\begin{tabular}{|c|c|c|c|}
\hline Element & $\begin{array}{l}\text { ICP-OES } \\
\mu \mathrm{g} / \mathrm{g}\end{array}$ & $\begin{array}{l}\text { ICP-MS } \\
\mu \mathrm{g} / \mathrm{g}\end{array}$ & $\begin{array}{l}\text { EDX } \\
\mu g / g\end{array}$ \\
\hline Silver (Ag) & n.d. & - & - \\
\hline Aluminium (Al) & $1006.2( \pm 100.9)$ & $1273.1( \pm 218.6)$ & - \\
\hline Arsenic (As) & n.d. & - & - \\
\hline Boron (B) & n.d. & - & - \\
\hline Barium (Ba) & $777.3( \pm 57.2)$ & $786.9( \pm 521.5)$ & $783.0( \pm 258.6)$ \\
\hline Bismuth (Bi) & n.d. & - & - \\
\hline Bromine (Br) & - & - & $30.0( \pm 4.9)$ \\
\hline Calcium (Ca) & $4639.2( \pm 1665.9)$ & - & $\mathrm{P}$ \\
\hline Cadmium (Cd) & $15.5( \pm 3.2)$ & $27.3( \pm 7.0)$ & $20.5( \pm 12.1)$ \\
\hline Chlorine (Cl) & - & - & $\mathrm{P}$ \\
\hline Cobalt (Co) & n.d. & $3.0( \pm 0.2)$ & - \\
\hline Chromium (Cr) & $116.3( \pm 24.0)$ & $179.6( \pm 19.7)$ & $58.8( \pm 10.5)$ \\
\hline Copper (Cu) & $24.3( \pm 2.9)$ & $51.9( \pm 4.3)$ & $\mathrm{P}$ \\
\hline Iron (Fe) & $1591.2( \pm 163.2)$ & $1835.2( \pm 472.0)$ & $\mathrm{P}$ \\
\hline Gallium (Ga) & $1.2( \pm 0.1)$ & - & - \\
\hline Mercury (Hg) & n.d. & - & - \\
\hline Indium (In) & $7.4( \pm 3.6)$ & - & - \\
\hline Potassium (K) & $392.8( \pm 35.9)$ & - & $\mathrm{P}$ \\
\hline Lithium (Li) & n.d. & - & - \\
\hline Magnesium (Mg) & $563.8( \pm 26.1)$ & - & - \\
\hline Manganese (Mn) & $27.3( \pm 2.9)$ & $35.2( \pm 11.8)$ & $\mathrm{P}$ \\
\hline Molybdenum (Mo) & $9.1( \pm 0.5)$ & $9.6( \pm 1.4)$ & $\mathrm{P}$ \\
\hline Sodium (Na) & $1043.0( \pm 66.6)$ & - & - \\
\hline Nickel (Ni) & $12.7( \pm 2.8)$ & $49.4( \pm 8.6)$ & $\mathrm{P}$ \\
\hline Phosphorus (P) & $166.0( \pm 9.8)$ & - & - \\
\hline Lead $(\mathrm{Pb})$ & $170.8( \pm 16.7)$ & $175.0( \pm 13.0)$ & $156.5( \pm 35.3)$ \\
\hline Palladium (Pd) & $19.3( \pm 6.7)$ & - & - \\
\hline Rubidium (Rb) & n.d. & - & $\mathrm{P}$ \\
\hline Rhodium (Rh) & n.d. & - & - \\
\hline Sulphur (S) & $681.7( \pm 49.8)$ & - & $\mathrm{P}$ \\
\hline Antimony (Sb) & n.d. & - & $14( \pm 2.3)$ \\
\hline Selenium (Se) & n.d. & - & n.d. \\
\hline Silicon (Si) & n.d. & - & $\mathrm{P}$ \\
\hline Tin $(S n)$ & $14.7( \pm 1.8)$ & - & $\mathrm{P}$ \\
\hline Strontium (Sr) & $28.0( \pm 6.8)$ & - & $\mathrm{P}$ \\
\hline Titanium (Ti) & $65.8( \pm 12.6)$ & - & $\mathrm{P}$ \\
\hline Vanadium (V) & n.d. & $3.2( \pm 0.6)$ & - \\
\hline Zinc (Zn) & $70.0( \pm 6.8)$ & $111.6( \pm 15.2)$ & $\mathrm{P}$ \\
\hline Zirconium (Zr) & n.d. & - & $\mathrm{P}$ \\
\hline
\end{tabular}


plastic additive chemicals such as flame retardants, plasticizers, colorants and inks. As this is a non-target screening approach, no standards were available for quantification, and the data presented are qualitative. Based on the chromatographic peak areas, compounds present at the highest concentrations were diisooctyl phthalate, tris(2-chloro-1-methylethyl) phosphate, dioctyl terephthalate, bis(3-chloro-1-propyl)(1-chloro-2-propyl)phosphate, and dibutyl phthalate.

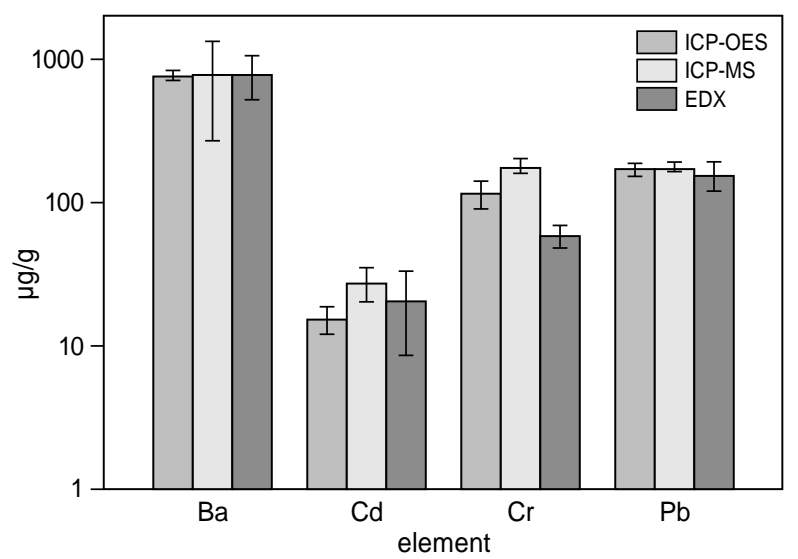

Figure 8.4. Comparison of elements measured in the MLD microplastic mixture with three different methods (ICP-OES, ICP-MS and EDX) with standard deviation.

\section{Discussion}

Collection of microplastic from the marine environment in quantities sufficient for use in laboratories studies is not feasible, and would involve extraction and clean up processes that may change physicochemical properties. Creating a representative mixture of marine microplastic from macroplastic marine litter offers an alternative approach to providing more environmentally relevant test materials. However, this approach is challenging and may be the reason why environmental samples are rarely been used in microplastic impact assessments (Phuong et al. 2016). Many factors play a confounding role, with the most prominent being the untraceable history of each marine litter item. Biological, mechanical and chemical degradation will differ for individual items, influencing their behaviour and composition (Gewert et al. 2015; Suhrhoff \& Scholz-Böttcher 2016; Booth et al. 2017). Local currents and bathymetry determine the type of marine plastic litter that arrives on a specific beach, which may be influenced by seasonal weather patterns (Schulz et al. 2013; Galgani et al. 2015; Watts et al. 2017). Furthermore, specific regions are likely to exhibit a unique composition of plastic litter reflecting the main local sources. For example, the percentage of fisheries-related plastic has been shown to vary per location, with the size of beached plastic also differing (Galgani et al. 2015). 
Table 8.5. Summary of organic chemicals present in the MLD microplastic mixture detected with GC-MS screening. Where available, CAS numbers, common names or abbreviations are given, otherwise $\mathrm{n} / \mathrm{a}$ is stated

\begin{tabular}{|c|c|c|c|c|}
\hline Compound Name & CAS No. & $\begin{array}{l}\text { Common Name/ } \\
\text { Abbreviation }\end{array}$ & $\begin{array}{l}\text { \% match } \\
\text { (NIST) }\end{array}$ & Comments \\
\hline 1,2,4-trimethylbenzene & $95-63-6$ & Pseudocumene & 80 & $\begin{array}{l}\text { Possible source from } \\
\text { printing inks }\end{array}$ \\
\hline $\begin{array}{l}\text { 1-Pentene, 4,4-dimethyl-1,3- } \\
\text { diphenyl-1-(trimethylsilyloxy) }\end{array}$ & $\mathrm{n} / \mathrm{a}$ & $\mathrm{n} / \mathrm{a}$ & 83 & \\
\hline 1-(4-methylphenyl)pentan-1-one & $1671-77-8$ & $\begin{array}{c}\text { 4'-methyl } \\
\text { valerophenone }\end{array}$ & 81 & \\
\hline 1-(1'-pyrrolidinyl)-2-butanone & $\mathrm{n} / \mathrm{a}$ & $\mathrm{n} / \mathrm{a}$ & 80 & \\
\hline 2,4-Di-tert-butylphenol & $96-76-4$ & 2,6-DTBP & 91 & UV stabiliser and antioxidant \\
\hline 2,4-dimethyldecane & $2801-84-5$ & $\mathrm{n} / \mathrm{a}$ & 84 & $\begin{array}{l}\text { Linked to production of } \\
\text { tributylphosphate plasticiser }\end{array}$ \\
\hline Tri(2-chloroethyl) phosphate & $115-96-8$ & TCEP & 84 & $\begin{array}{l}\text { Flame retardant, plasticiser } \\
\text { and viscosity regulator }\end{array}$ \\
\hline 3,7-dimethylnonane & $17302-32-8$ & $\mathrm{n} / \mathrm{a}$ & 81 & \\
\hline $\begin{array}{l}\text { Tris(2-chloro-1-methylethyl) } \\
\text { phosphate }\end{array}$ & $13674-84-5$ & ТСРP & 93 & Flame retardant \\
\hline $\begin{array}{l}\text { Bis(3-chloro-1-propyl)(1-chloro-2- } \\
\text { propyl)phosphate }\end{array}$ & $137888-35-8$ & $\mathrm{n} / \mathrm{a}$ & 86 & Flame retardant \\
\hline Dibutyl phthalate & $84-74-2$ & DBP & 94 & $\begin{array}{l}\text { Plasticiser or possible } \\
\text { printing ink }\end{array}$ \\
\hline Triphenyl phosphate & $115-86-6$ & $\mathrm{TPhP}$ & 87 & Plasticiser and flame retardant \\
\hline 2-Ethylhexyl diphenyl phosphate & $1241-94-7$ & Octicizer & 80 & Plasticiser and flame retardant \\
\hline $\begin{array}{l}\text { Phthalic acid, } \\
\text { di(6-methylhept-2-yl) ester }\end{array}$ & $\mathrm{n} / \mathrm{a}$ & $\mathrm{n} / \mathrm{a}$ & 80 & Plasticiser \\
\hline Dicyclohexyl phthalate & $84-61-7$ & Morflex $^{\circledR} 150$ & 84 & Plasticiser and UV stabiliser \\
\hline Bis(2-ethylhexyl) phthalate & $117-81-7$ & DEHP & 96 & $\begin{array}{l}\text { High volume production } \\
\text { plasticiser }\end{array}$ \\
\hline Diisooctyl phthalate & $27554-26-3$ & DIOP & 93 & $\begin{array}{l}\text { High volume production } \\
\text { plasticiser or possible printing } \\
\text { ink. Also used as glue in } \\
\text { PU- PVA-based colour binders }\end{array}$ \\
\hline 5-methyl-2-phenyl-1H-indole & $13228-36-9$ & $\mathrm{n} / \mathrm{a}$ & 80 & $\begin{array}{l}\text { Indoline substances are used } \\
\text { as colorants }\end{array}$ \\
\hline $\begin{array}{l}\text { 2-tert-Butyl-6-(5-chloro-2H- } \\
\text { benzotriazol-2-yl)-4-methylphenol }\end{array}$ & $3896-11-5$ & Bumetrizole & 86 & $\begin{array}{l}\text { Antioxidant used to slow the } \\
\text { oxidation process of the } \\
\text { polymer exposed to UV light }\end{array}$ \\
\hline $\begin{array}{l}\text { 1,4-Benzenedicarboxylic acid, } \\
\text { bis(2-ethylhexyl) ester }\end{array}$ & $6422-86-2$ & $\begin{array}{l}\text { Dioctyl } \\
\text { terephthalate }\end{array}$ & 92 & $\begin{array}{l}\text { Non-phthalate plasticiser used } \\
\text { in PVC, PMMA and PS }\end{array}$ \\
\hline (Z)-9-Octadecenamide & $301-02-0$ & Oleamide & 80 & $\begin{array}{l}\text { Lubricant in PE and } \\
\text { PP manufacture }\end{array}$ \\
\hline
\end{tabular}


The number of variables influencing plastic litter at specific locations has so far prevented its use in experimental research. However, the use of macroplastic litter for the generation of more environmentally relevant microplastic reference materials appears to be a necessary step in closing the current gap between experimental lab studies and observations made in the natural environment (Jahnke et al. 2017). Despite these potential challenges, this study serves as a first step and a suggested method for creating and characterising a MLD microplastic mixture is presented. The current study shows there are still issues of concern throughout all stages of the process.

The use of macroplastic litter data from a previous study (Van Franeker 2005) enabled collection of new plastic litter items that reflected the original composition determined in the 2005 study (Table 8.1). However, generation of a microplastic mixture reflecting the composition of marine litter requires a detailed assessment and categorisation of marine litter in the sampling area. It is important to note that care needs to be taken to ensure items exhibit some degree of weathering or biofouling that suggests a prolonged residence time in the marine environment. Furthermore, any resulting microplastic mixture represents only the area from which the source litter was collected and may not be representative globally. In the current study, PE and PP were the most abundant polymer types in the collected beach litter (Figure 8.3), possibly reflecting the lightweight nature of these materials and that they are the highest volume production plastics globally (Geyer et al. 2017). Many studies have shown that PE and PP are the dominant polymers in global marine debris (Hidalgo-Ruz et al. 2012), in North Atlantic sediments (Fries et al. 2013; Sadri \& Thompson 2014; Claessens et al. 2011) and in different species ingesting plastics in the North Sea (Foekema et al. 2013; Rummel et al. 2016; Van Franeker et al. 2018).

The cryogenic milling approach employed in the current study can successfully produce microplastic from macroplastic litter. However, laboratory-scale cryogenic milling is only suitable for small samples, so the raw materials were cut to an acceptable size $(<25$ $\mathrm{mm}$ ). Furthermore, some loss of the material was observed during the milling process, with rigid marine litter items exhibiting higher recoveries than materials with a small volume/mass ratio (e.g. sheets and foams). As foil and foams were observed to become statically charged under milling, it was necessary to mill specific categories of materials individually before combining and homogenising into a final microplastic mixture with the target polymer composition. Each step of the microplastic mixture preparation was implemented in a way to try and minimise any change to the chemical composition of the sample (e.g. use of cryogenic system), therefore ensuring that the chemical profile of the final microplastic mixture resembled that of the source marine litter as closely as possible.

By subjecting the bulk microplastic mixture to a serial sieving process, a mass distribution of the different size fractions could be determined (Figure 8.1). Results showed that cryogenic milling successfully reduced the macroplastic litter into microplastic, with 95\% of the recovered particulates by mass being $<2.0 \mathrm{~mm}$ in size. The PSD of the microplastic mixture showed that the number particles present in each of the size fractions increased significantly as particle size decreased. Fitting to a basic exponential function: $(y=a * x$ $\wedge-b)$ and to the logarithm of the same function suggests the relationship between 
particle size and particle number is exponential (but break down for the highest size categories; Online Supplement 3, Figure 3.1.). Interestingly, the particle distribution of the different categories varies for the different size fractions (Table 8.3). The number of sheet, thread and fragment particles increases as the diameter of the size fraction decreases. Conversely, the number of foam and dust particles decreases with decreasing size fraction, suggesting that individual materials behave differently during the milling process. In the largest size category $(>4.5 \mathrm{~mm}$ ) only dust balls and aggregated conglomerates were observed, showing that cryo-milling can be useful to create a microplastic mixture $(<5$ $\mathrm{mm}$ ). However, the variation in composition observed in the different size fractions may restrict the use of specific size fractions in certain studies, as the composition does not reflect the original composition on beaches.

SEM images of the microplastic mixture indicate all particles exhibited an irregular shape (Figure 8.2). Brittle plastic litter items fragmented into irregular shaped particles, while other particles showed evidence of stretching, tearing and crushing rather than 'clean' fragmentation. Despite the use of cryogenic cooling, specific material types clearly respond differently in the milling process. The irregular shape of the particles also influenced the efficiency of the size fractionation processes. High aspect ratio particles (e.g. fibres) were found to distribute between different size fractions as a small number could pass through the smaller sieves if the particle was orientated the correct way. Nevertheless, the results of comparable but independent chemical analyses (FTIR-ATR and DSC) indicate that a satisfactory homogenization of the mix is possible. The majority of plastic items analysed consisted of PE and PP (61 and 28\% respectively). PE and PP are the most commonly produced polymer materials globally (Geyer et al. 2017), which appears to be reflected in the results of the current study. Both polymer types have a lower density than seawater and tend to float on the water surface as long as they are not heavily bio-fouled or vertically mixed by wind and wave action (Kooi et al. 2017; Kukulka et al. 2012). Thiel et al. (2013) observed a comparable plastic litter compositions in coastal waters and on beaches, suggesting beached material can be used as a proxy for that in surface waters. The MLD microplastic mixture may also be a suitable reference material for plastic below the surface, as plastic ingested by fish in the same region consisted mainly of PE and PP (Rummel et al. 2016; Collard et al. 2015; Collard et al. 2017).

Elemental analysis of the microplastic mixture indicated a broad range of heavy metal, light metal and metalloid elements associated with the particles. Reproducibility was good between replicate sub-samples of the microplastic mixture, indicating that the final homogenisation step had been successful. The elemental distributions and concentrations determined in the current study were broadly similar with all three techniques employed, indicating successful homogenisation and suggesting the 3-method design reduces the need for using certified reference materials. HF digestion combined with ICP-MS analysis offers the most accurate and sensitive approach, but is more time consuming and costly. EDX offers a good screening method for the presence of many elements, including halogens such as bromine and chlorine which are common in flame retardants, but quantification is difficult as crystallisation and polymer composition affect the result and detection 
limit. For routine identification and quantification of microplastic elemental composition, ICP-OES appears to offer the best combination of sensitivity and cost.

Many elements are well-known inorganic plastic additives and their industrial sources can be postulated. Other elements may be residual contaminants from the polymerisation process (Crompton 2007; Brennecke et al. 2016; Turner 2016). Inorganic plastic additives are typically utilised as fillers ( $\mathrm{Ba}, \mathrm{Ca}, \mathrm{Mg}$ ), colorants ( $\mathrm{Cd}, \mathrm{Fe}, \mathrm{Mn}, \mathrm{Pb}, \mathrm{Ti}$ ) or stabilisers/catalyst left overs/ inks ( $\mathrm{Cu}, \mathrm{Mo}, \mathrm{P}, \mathrm{Pb}, \mathrm{Sn}, \mathrm{Sr}, \mathrm{Zn}$ ). Al is commonly added to plastic foils to improve the barrier function in products such as food packaging or nonlatex helium balloons, whilst $\mathrm{Ba}$ is also used to prevent discoloration or yellowing in $\mathrm{PE}$ foils. The presence of such elements in microplastic is rarely considered, and the relationship between plastics and metals remains unclear (Brennecke et al. 2016). For example, Fries et al. (2013) suggest that $\mathrm{Al}$ and $\mathrm{Zn}$ present in their samples was assumed to be sorbed from the surrounding environment. Kedzierski et al. (2018) observed only few heavy metals adsorbing to the surface of PVC after being exposed to seawater a prolonged period. Importantly, certain elements are known to be toxic to a wide range or marine organisms, so quantifying their presence in microplastic test materials is necessary when used in ecotoxicity studies.

Using analysis techniques that include a digestion step (e.g. ICP-OES and ICP-MS) mean it is not possible to distinguish between elements from the manufacturing process and those adsorbed to the surface from the environment. As the microplastic mixture in the current study is derived from weathered macroplastic litter, it is possible that the elemental composition represents a combination of sorbed and additive substances. However, it is not possible to accurately identify the sources of the different elements identified within the microplastic mixture.

Organic chemical screening by GC-MS indicated a range of additive chemicals were present in the microplastic mixture, with phthalates and chlorinated alkyl phosphate esters (organophosphorus compounds) being two of the most dominant chemical classes (Table 8.5). Diisooctyl phthalate (DIOP) and dibutyl phthalate are plasticisers mainly related to PP and PE products, and have previously been identified in marine debris (Fries et al. 2013; Rani et al. 2015). DIOP and dibutyl phthalate are known endocrine disruptors (Oehlmann et al. 2009). Plasticisers are often related to polyvinyl chloride where up to $60 \%$ of the material mass may originate from this type of additive. However, they have also been found in printing inks and lacquers used in the production of many other polymer types (Fierens et al. 2012), and is suggested as their most likely source in the MLD microplastic mixture. Dicyclohexyl phtalate (DCHP) has been found in low concentrations in food packaging (Fierens et al. 2012; Schecter et al. 2013). Tris(2-chloro-1methylethyl) phosphate (TCPP), together with many other organophosphorus compounds, is regularly used as an additive flame retardant and is a suspected carcinogen (Andresen et al. 2004). High levels of Bis(2-ethylhexyl) phthalate (DEHP) and phthalic acid, di(6-methylhept-2-yl) ester have been found in artificially aged rubber ducks, in contrast to low or no levels in unaged ducks (Cambridge Polymer Group 2016). These substances might be breakdown products of the weathering process, although the exact 
mechanisms behind the changed levels remain unclear.

The toxicity of many additive organic compounds to marine and freshwater species has been studied previously (Adams et al. 1995; Chen et al. 2014). For example, the acute toxicity of a group of 14 phthalate esters to representative aquatic organisms was found to range from 0.21 to $377 \mathrm{mg} / \mathrm{L}$ depending on the ester and the solubility of the test chemical in water (Adams et al. 1995). A general trend was found showing that lower-molecular-weight phthalate esters become more toxic with decreasing water solubility, but there were only minor differences in species sensitivity to each of the phthalate esters. Although the production, sources and environmental occurrence of organophosphorus compounds has been reviewed extensively, there is currently limited data available regarding the toxicity this class of compounds to aquatic organisms (van der Veen \& de Boer 2012; Wei et al. 2015). The limited data available suggests there may be significant differences in the toxicity of different compounds within the class (van der Veen \& de Boer 2012).

EDX identified the presence of bromine and chlorine in the microplastic mixture (Table 8.4), but the technique could not determine if the signal derived from sea salt residues or from additive chemicals (e.g. flame retardants). Although halogenated flame retardants were not specifically studied owing to the non-target GC-MS screening approach employed, the analysis confirmed the presence of chlorinated compounds (Table 8.5). Brominated compounds were not observed, suggesting they are present at very low concentrations or absent from the sample. In a study of marine litter items by Turner \& Lau (2016), comparable values for bromine (and most other elements) were detected in non-porous fragments and ropes, but were much higher in foam $(20,26$ and $244 \mu \mathrm{g} / \mathrm{g}$, respectively). It remains unclear whether these substances were added during production processes or were adsorbed when exposed to seawater, as demonstrated in a long-term exposure experiment by Rochman et al. (2014). Regardless the origin, some of the substances have been described as hazardous (Lithner 2011), and therefore pose a potential risk to marine organisms (Rochman et al. 2014; Turner \& Lau 2016).

It is important to note that the concentrations of inorganic and organic substances associated with the cryo-milled microplastic mixture in the current study may differ from those in true microplastic particles that are the result of natural degradation and subject to potential desorption-adsorption processes in the marine environment. In the milled sample, much of the surface of the resulting microplastic will not have been subjected to the marine environment and may not contain the same levels of adsorbed metals and organics. Conversely, the fresh surface area generated has not had the opportunity to release any additive chemicals to the surrounding marine environment. However, marine organisms as the Northern Fulmar that ingest and grind plastic particles in their muscular stomach (Van Franeker et al. 2011) might be exposed to both additives and adsorbed pollutants.

\section{Conclusion}

There is a need to move towards using more environmentally relevant microplastic reference materials in laboratory-based effects assessment. The microplastic mixture generated 
in the current study represents a significant step forward from the use of pristine spherical materials typically used in laboratory studies, especially in terms of physical properties. The generated mixture will be distributed between partners of the JPI Oceans PLASTOX project for laboratory effects assessment studies. As the collection, preparation and characterisation of the MLD microplastic mixture is time consuming, and true reproducibility is impossible, it is currently not feasible to supply the MLD microplastic mixture commercially. However, this study should act as a guide for others to generate relevant MLD microplastic mixtures that are representative of specific marine environments.

There will always be some differences in the chemical profile of the milled material relative to naturally degraded microplastic particles present in the marine environment. Irrespective of the origin of the inorganic and organic substances associated with microplastic in the marine environment (additives or sorbed), it represents a cocktail of substances that may elicit impacts in marine organisms. As we move more towards sublethal effects studies to understand the environmental impacts of plastic pollution, the need to study the role and possible contribution of additive chemicals is crucial. It is therefore recommended that any microplastic test material used in effects studies, regardless of its origin, is subjected to a detailed physicochemical characterization so that effects data may be interpreted more accurately. The application of multiple, complimentary analytical chemical and imaging techniques appears essential for characterising microplastic test materials.

\section{Acknowledgements}

Susanne Kühn is funded through the Joint Program Initiative (JPI) Oceans PLASTOX (Direct and indirect ecotoxicological impacts of microplastics on marine organisms) project by the Netherlands Organisation for Scientific Research (NWO) under the project number ALW-NWO 856.15.001. SINTEF Ocean was funded through the JPI Oceans project PLASTOX by Research Council of Norway (RCN; Grant Agreement number 257479/E40). The authors wish to thank the NWO and the RCN for their financial support. We are very grateful for the assistance in the labs provided by Markus Ortlieb (Shimadzu), Marianne Kjos and Lisbet Sørensen (SINTEF) as well as Andre Bouwmeester, Stephanie Westerhoff and Erwin Jansen (Carat GmbH). 


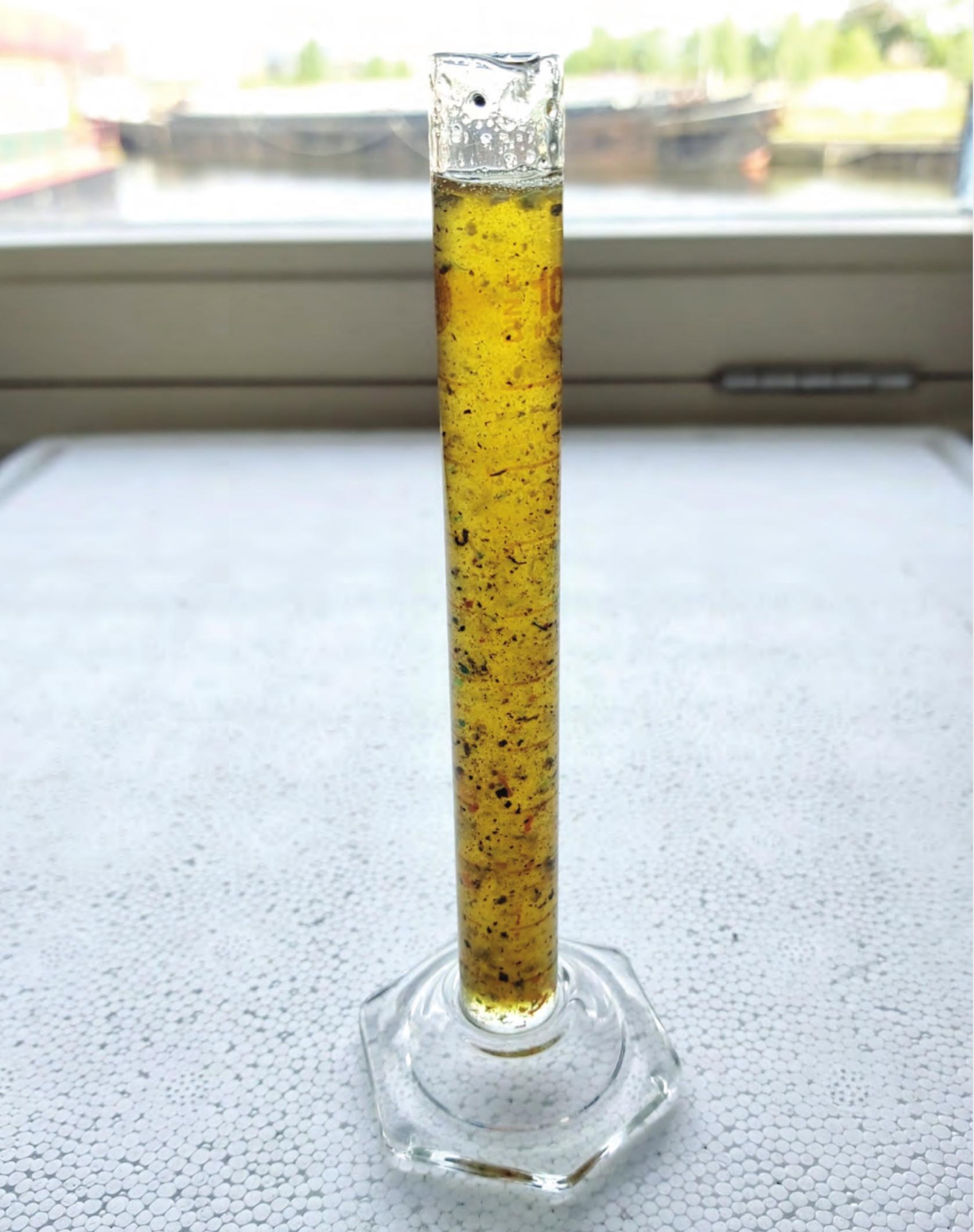




\section{Transfer of additive chemicals from marine plastic debris to the stomach oil of northern fulmars}

Susanne Kühn,

Andy M. Booth,

Lisbet Sørensen,

Jan A. van Franeker 


\begin{abstract}
For this study, the transfer of plastic additives to stomach oil of northern fulmars (Fulmarus glacialis) has been investigated. Stomach oil is a substance produced by procellariform seabirds from dietary remains, used as energy storage. A marine litter-derived microplastic reference mixture and separately a litter-derived polystyrene sample were used. A total of 14 additives, including plasticizers, antioxidants, UV stabilizers, flame retardants, and preservatives were detected in the original plastic mixture. These substances are known for negative effects on organisms. Stomach oil was exposed to these plastic materials and were sampled during a pilot study $(0,14$ and 90 days) and a subsequent short-term full experiment ( 8 hours, 1, 2, 4, 8 and 21 days) that included re-analysis of the earlier 14- and 90- day samples. The plastic mixture showed signs of leaching in five substances, four substances did not appear to leach while the remaining substances showed an unclear pattern. Four substances were detected in the polystyrene foam, two of them leaching into stomach oil. For unclear reasons, the results of the pilot study and the full experiment were not fully comparable, but control levels and replicates show reliable data output in most substances. Leaching of potentially harmful plastic additives to the stomach oil of fulmars should be of concern, as fulmars ingest plastics regularly and grind them down in the gizzard before passage to the intestines and excretion.
\end{abstract}




\section{Introduction}

Northern fulmars (Fulmarus glacialis) are seabirds of the tubenosed family (Procellariiformes). They are non-specialized foragers, opportunistically hunting for fish, squid, crustaceans and jellyfish at or close to the seawater surface (Camphuysen \& Van Franeker 1997; Ojowski et al. 2001; Byrkjedal \& Langhelle 2019), but also scavenging on ship offal and carrion (Camphuysen \& Garthe 1997). In contrast to other seabirds, most procellariform store energy reserves not only in adipose fat, but also in oil, accumulated in the proventriculus of the bird (Wang et al. 2007). This light yellow to dark orange coloured oil is produced from dietary remains and is not a product of stomach excretions (Lewis 1969; Clarke \& Prince 1976; Imber 1976). The composition of stomach oils can vary greatly depending on species and the most recent diet, but typically consists of different types of wax esters, diacyl glycerol ethers and triglycerides (Lewis 1969; Imber 1976). Stomach oil is found in all life stages of procellariform seabirds and is an efficient way to store highly concentrated caloric food in low volumes. This is more efficient and corresponds more flexibly to the energy demands of the birds as metabolism of fatty acids to the adipose fat reserves is unnecessary (Place et al. 1989).

Seabird stomach oil has been suggested as a suitable monitoring medium for marine pollution, as it accumulates hydrophobic organic pollutants such as petroleum hydrocarbons (Clarke \& Prince 1976) and PCBs (Foster et al. 2010), the latter obtained via contaminated natural food. Beside pollutants, acquired via seawater and prey, fulmars regularly ingest plastics in high quantities. In the North Sea, $93 \%$ of the fulmars contain on average 33 plastic particles, with an average mass of 0.31 gr (OSPAR 2017). These numbers decrease with latitude and reflect local abundances of plastics in the environment (Van Franeker et al. 2011; Kühn \& Van Franeker 2012; Trevail et al. 2015a; Mallory 2008).

Plastic debris can contain a broad range of additive chemicals and existing environmental pollutants, raising questions about the bioavailability of these chemicals following ingestion by marine organisms. There are two main pathways in which these chemicals are associated with plastic debris. First is the addition of additive chemicals during the production process to enhance specific characteristics of the plastics (e.g. flexibility, flame resistance or colour) and residual chemicals from this production process. The other pathway occurs when plastics are exposed to the (marine) environment (Teuten et al. 2009). Substances can be adsorbed to the plastic surface, especially smaller plastic items which are prone to such process as they exhibit a comparably larger surface than volume. Weathering of plastics in the marine environment may enhance this adsorption process (Jahnke et al. 2017).

The combination of both pathways, together with the physical characteristics of plastic may harm marine wildlife when these plastics are ingested. The capacity for wildlife to take up a range of plastic associated chemicals has been demonstrated in laboratory experimental setups (summarized by e.g. Teuten et al. 2009; Browne et al. 2015). However, unrealistic scenarios are often applied during experiments with regard to the of type and 
shape of plastic, degree of degradation and associated toxic substances, as most studies use homogeneous shaped, pristine plastics (Phuong et al. 2016).

In 2015, the uptake of plastics by marine organisms had been reported in at least 331 species (Kühn et al. 2015). Procellariform seabirds in particular were found to regularly ingest plastics, possibly confusing them with natural diet items. Out of 141 procellariform seabird species, 60\% have been recorded with plastics (Kühn et al. 2015), sometimes in frequencies of occurrence higher than 90\% (e.g. Van Franeker et al. 2011; Roman et al. 2016; Rapp et al. 2017; Rodríguez et al. 2018). Procellariiformes take up a wide variety of plastics, including different shapes (van Franeker et al. 2011) and colours (Kühn et al. 2015), with the size of the ingested plastics related to body size (Roman et al. 2019c).

After swallowing, the first prolonged contact of ingested plastic with procellariform seabirds occurs in the proventricular stomach. The retention time of plastic in seabirds is unknown and may vary per species and by type of plastic item (size, shape, flexibility). For the northern fulmar and close relatives, Van Franeker \& Law (2015) suggest that within a month, $75 \%$ of the ingested plastic can be ground down to dust, small enough to pass into the intestines and to be excreted. However for other species, retention times of several months to even years have also been reported (Ryan 2015a). Depending on the size of the ingested items, plastics (and natural food) are first retained in the large expandable proventriculus where gastric juices are produced to initiate the digestion process. Once in the smaller and muscular gizzard, plastic items are ground down by strong stomach walls, before grounded dust or broken particles small enough to pass to the intestines can be excreted. In northern fulmars, hard plastic particles have to be reduced to just a few millimetres in size before the can disappear from the gizzard (Bravo Rebolledo 2011; Terepocki et al. 2017).

The grinding process increases the surface of ingested plastics in the stomach. Consequently, more additive chemicals that have been incorporated in the plastic are available for an exchange with the stomach oil. This process has been described previously by Tanaka et al. (2015), where the uptake of polybrominated flame retardants to stomach oil of procellariforms has been recorded.

The current study investigated the uptake of plastic additive chemicals into fulmar stomach oil, using plastic debris from the marine environment. Plastic debris was collected from a Dutch beach and carefully characterised in terms of polymer type, shape and size and milled into a microplastic mixture (Kühn et al. 2018b). In addition, a comparable sample was compiled with beached polystyrene foam only. The additive chemical profile of the materials was analysed by chemical extraction followed by non-target screening analysis by gas chromatography mass spectrometry. Stomach oil was exposed to these plastics under realistic circumstances and after exposure analysed to determine the chemical content. The mechanisms of uptake of substances from plastic into seabirds are a crucial step to understand potential harm of plastic on seabirds, not only on individual but also on population level. 


\section{Methods}

\section{Plastic source materials}

A marine litter-derived microplastic reference material (PTX001) has been used. The collection and processing of the material has been described in detail in Kühn et al. (2018b). Briefly, 351 macroplastic litter items were collected from a Dutch beach, equalling the mass composition of plastics during earlier beach-clean-ups. The collected material was cryomilled (Retsch ZM 200, Carat $\mathrm{GmbH}$ ) to a mix of variable sizes under 3.0 $\mathrm{mm}$ diameter. One gram of the PTX001 material contained around 400,000 plastic particles. The produced microplastics are irregular in size and shape, being more realistic than the uniform spherules often used in exposure experiments. The polymer composition comprises mainly polyethylene (PE) and polypropylene (PP), with many other polymers present similar in distribution to polymers produced on a global scale (Geyer et al. 2017) and those found in seabirds (e.g. Tanaka et al. 2019). Chemical analyses confirmed the abundance of various elements, as well as chemical substances associated to plastic production processes. Different plasticisers, UV stabilizers and flame retardants have been found in the mixture (Kühn et al. 2018b). To determine the contribution of different plastic types to the total process of leaching, three pieces of weathered expanded polystyrene foam (PS) were collected from the same beach as the macroplastic litter items used in production of the PTX001 mixture. The PS foam was cut manually to particles of approximately $0.5 \mathrm{~mm}$ in size.

\section{Stomach oil preparation}

Stomach oil was collected from northern fulmars on the Faroe Islands in the north Atlantic Ocean, where fulmar chicks are harvested for human consumption (Jensen 2012). The oil was drained directly from the stomach to glass bottles and frozen at $-20^{\circ} \mathrm{C}$. The stomach oil used in the current study was combined from more than 50 different chicks collected between 2014 and 2016. As natural foods may contain contaminants, and as chicks already contain plastics, transferred by their parents, a basic load of chemicals in to stomach oil is to be expected already at the start of the experiments.

\section{Experimental setup}

Two exposure experiments were conducted; a pilot study with few widely separated sampling points at up to 90 days, and a full study with a denser schedule of time points up to 21 days. The procedure and setting for both studies were the same and the same stomach oil and plastics test materials were used in all studies. Stomach oil was exposed to the two different microplastic test materials (PTX001 and PS) for varying amount of times depending on the study regime employed. Plastics were added to the stomach oil and stirred continuously in a shaking bath (Julabo SW23) at $120 \mathrm{rpm}$ to mimic stomach contractions at $40^{\circ} \mathrm{C}$, the common body temperature in procellariform seabirds (Warham 1996). The exposure design comprised of two bottles with controls without added plastic, two bottles of the microplastic mixture (PTX001) and two bottles of PS. Each bottle 
contained $40 \mathrm{~mL}$ of stomach oil and the added quantity of plastics were $1.0 \mathrm{~g}$ for the PTX001 samples (25 g/L), and $0.3 \mathrm{~g}$ for the PS foam $(7.5 \mathrm{~g} / \mathrm{L})$. At each sampling time point, $5 \mathrm{~mL}$ of oil was removed from each bottle, in which care was taken to ensure oil and plastics were removed in proportional quantities. The oil was then vacuum-filtered through a glass microfibre filter (GFF, pore size $0.7 \mu \mathrm{m}$; GE Whatman) in a glass filtration system to remove the plastic particles (Figure 9.1). The filtered oil from each $5 \mathrm{~mL}$ sample was then divided over three glass vials of approximately $1 \mathrm{~mL}$ each, and directly frozen at $-20^{\circ} \mathrm{C}$ until further analysis. Thus, finally each original sample resulted in 6 samples available for chemical analysis: each of two sample bottles of $40 \mathrm{~mL}$ (A and B) at each time point divided over three $\pm 1 \mathrm{~mL}$ test vials). All laboratory materials and Teflon capped sample containers were carefully rinsed in hexane before being used.

In the long-term pilot study, samples were collected at the beginning of the experiment at day 0 , and next after 14 and 90 days. On the basis of the analyses of these samples, a refinement on time-scale was implemented during the full study, where focus was on the first three weeks and samples were taken after 8 hours, 1, 2, 4, 8 and 21 days.

\section{Sample analysis}

In total, three sets of chemical analyses were conducted:

- The 'pilot study': measured are days 0, 14 and 90.

- The 're-analysis': the 14- and 90- day samples from the pilot study were reanalysed with the GC-MS methods used in full experiment

- The 'full experiment' repeated the pilot study with adapted sampling times $(0.3,1,2$, 4, 8, 21 days) and adapted GC-MS methods.

\section{Plastic extraction}

Samples of the original plastic materials ( $~ 500 \mathrm{mg}$ PTX001 mixture, $\sim 18-30 \mathrm{mg}$ PS foam) were subjected to solvent extraction with two different solvents. Three replicates of each material were extracted using dichloromethane (DCM, Rathburn) and three replicates were extracted using ethyl acetate (EtOAc, Fluka). In each case, $4 \mathrm{~mL}$ solvent and an internal standard mixture $(0.2508 \mu \mathrm{g}$ naphthalene- $d 8,0.0500 \mu \mathrm{g}$ phenanthrene- $d 10$, $0.0486 \mu$ g chrysene- $d 12$ ) was added to each sample prior to bath sonication for 30 minutes (Bandelin Sonorex Super RK 510H, 640W, $35 \mathrm{kHz}$ ) at either room temperature (DCM) or $65^{\circ} \mathrm{C}$ (EtOAc). The solvent extract was then filtered through a pipette packed with Bilsom cotton and a small amount of anhydrous $\mathrm{Na}_{2} \mathrm{SO}_{4}$ to remove plastic particles. The extracts were then concentrated by solvent evaporation $\left(40^{\circ} \mathrm{C}\right.$ under a gentle flow of $\mathrm{N}_{2}$ ) to approximately $500 \mu \mathrm{L}$ and a recovery internal standard (0.0984 $\mu$ g fluorene- $d 10$ and $0.1064 \mu \mathrm{g}$ acenapthene-d10) was added prior to GC-MS analysis. Prior to clean-up by gel permeation chromatography (GPC), samples extracted by DCM were re-adjusted to 1 mL volume with additional DCM.

\section{Stomach oil extraction}

From the $1 \mathrm{~mL}$ vials, samples of fulmar oil $(50 \mathrm{mg}$ in the pilot study and $100 \mathrm{mg}$ in the full 
study) were transferred to a glass tube and dissolved in $1 \mathrm{~mL}$ DCM: $n$-hexane (1:1). An internal standard mixture $(0.2508 \mu \mathrm{g}$ naphthalene- $d 8,0.0500 \mu \mathrm{g}$ phenanthrene- $d 10$, $0.0486 \mu \mathrm{g}$ chrysene- $d 12$ ) was added and the sample vortexed (30 seconds). Sample volume was adjusted to $1 \mathrm{~mL}$ by solvent evaporation $\left(40^{\circ} \mathrm{C}\right.$ under a gentle flow of $\left.\mathrm{N}_{2}\right)$.

\section{Gel permeation chromatography}

Both fulmar oil extracts and DCM polymer extracts were subject to instrumental clean-up by GPC (Agilent). Samples $(500 \mu \mathrm{L})$ were injected with DCM as the mobile phase (0.5 $\mathrm{mL} / \mathrm{min}$ for the pilot study, $5 \mathrm{~mL} / \mathrm{min}$ for the full study) and subject to separation using either an Agilent PLGel column $(7.5 \times 300 \mathrm{~mm}, 5 \mu \mathrm{m}$; pilot study) or a Waters Envirogel column $(19 \times 300 \mathrm{~mm}, 15 \mu \mathrm{m}$; full study). Chromatograms were monitored at 210, 254 and $280 \mathrm{~nm}$ UV. After initial optimization, analyte fractions were collected from 16-35 minutes (pilot study) or 10.5-15 minutes (full study) with pre-added $n$-hexane in the collection vials as a keeper. The sample volume was adjusted to $0.5 \mathrm{~mL}$ by solvent evaporation $\left(40^{\circ} \mathrm{C}\right.$ under a gentle flow of $\left.\mathrm{N}_{2}\right)$ and recovery internal standards $(0.0984 \mu \mathrm{g}$ fluorene- $d 10$ and $0.1064 \mu \mathrm{g}$ acenapthene- $d 10$ ) were added prior to analysis by GC-MS.

\section{GC-MS fullscan analysis}

The GC-MS system comprised an Agilent 7890A GC equipped with an Agilent 5975C Mass Selective Detector (MSD). The inlet was set to $250^{\circ} \mathrm{C}$, the transfer line to $300^{\circ} \mathrm{C}$, the ion source to $230^{\circ} \mathrm{C}$ and the quadrupole to $150^{\circ} \mathrm{C}$. The carrier gas was helium, at a constant

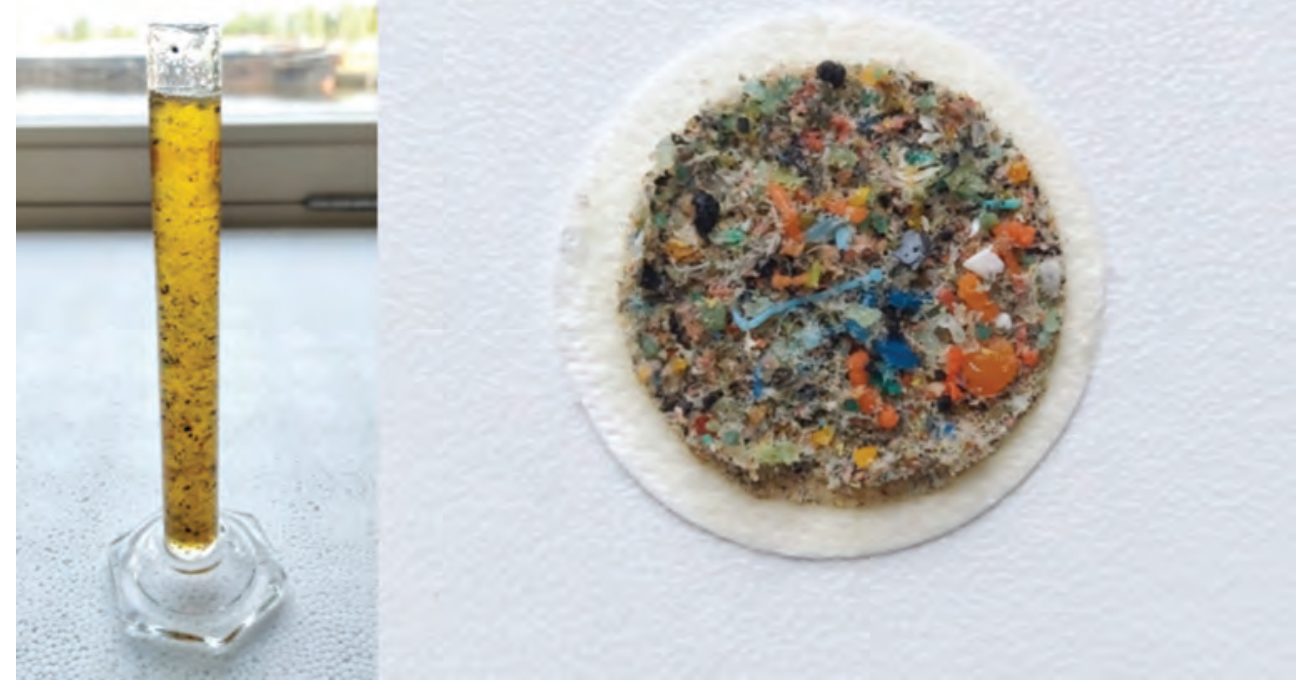

Figure 9.1. Left: Marine derived plastic litter (PTX001) in stomach oil before filtration. Right: PTX001 on a GFF filter (diameter $47 \mathrm{~mm}$ ) after filtration. Note different plastic shapes, sizes and colours. 
flow of $1.1 \mathrm{~mL} / \mathrm{min} .1 \mu \mathrm{L}$ of sample was injected by pulsed splitless injection. The GCcolumn was an Agilent DB5-MS ultra-inert column $(30 \mathrm{~m}, 0.25 \mu \mathrm{m}$ film thickness, 0.25 $\mathrm{mm}$ internal diameter). The GC oven was held at $40^{\circ} \mathrm{C}$ ( 2 minute), ramped by $6^{\circ} \mathrm{C} / \mathrm{min}$ to $320^{\circ} \mathrm{C}$ (20 min hold). Mass spectra were recorded in full scan mode after a 12 minutes hold time, $50-500 \mathrm{~m} / \mathrm{z}$.

\section{GC-MS selected ion monitoring (SIM) analysis}

Using compounds identified from the full-scan analysis of the PTX001 and PS material extracts, a selected ion monitoring (SIM) method was developed to enable a more detailed, targeted analysis of chemicals present in the stomach oil extracts. This approach increases the sensitivity of the analysis and helps to reduce background noise and interference from biogenic compounds derived from the stomach oil. The same GC-MS system and instrumental conditions as above were applied. Selected ions representative of tentatively identified compounds were monitored according to Table 1 in the Online Supplement.

\section{Data treatment}

For non-target screening, chromatograms and mass spectra were recorded using Chemstation software, investigated in Masshunter Qualitative Navigator B.08.00, further processed using Masshunter Unknowns Analysis ("Unknowns") followed by export to csv format using Python and data processed in R. After initial inspection of chromatograms, peaks were deconvoluted using Unknowns algorithms and best hits from NIST 2017 library were extracted. Compounds were filtered based on observed presence in at least 3 of 6 replicates for each polymer and $>90 \%$ match to NIST 2017 library mass spectra. Biogenic compounds, or compounds of possible biogenic origin were removed from the data set. All compounds found in control samples were also removed from the data set, leaving only those that could be confidently attributed to coming from the PTX001 and PS materials.

For targeted analysis, tentatively identified compounds were recorded by their retention time and major ions (Online Supplement Table 1) in GC-MS selective ion monitoring (SIM) mode. Masshunter Quantitative Analysis was further used to integrate peak areas of the selected compounds and the added internal standards. The area of each tentatively identified compound was normalised by dividing by the area of one internal standard in each sample and the normalised relative intensities used to compare samples.

\section{Control-derived limits of detection}

Given the exploratory and non-quantitative nature of the analysis, there was a lack of reference standard chemicals for the identified additives and therefore it was not possible to establish individual calibration curves for each chemical. Control-derived limits of detection (LOD) were established based on control measurements for each of the three treatments separately. The LOD was calculated as the average of the controls of each treatment +3 standard deviations. As evidenced by the results, however, even the unexposed control stomach oil samples contained some level of additive chemicals. 


\section{Results}

\section{Characterisation of plastic material}

With selected ion monitoring, 15 substances were confidently identified ( $>90 \%$ match to NIST 2017 library) in the PTX001 and PS material. These substances include common additives such as plasticizers, antioxidants, UV stabilizers, flame retardants, and preservatives. However, for some chemicals identified in the samples the use or origin is unclear. All substances, except phenyl benzoate, were detected in the original PTX001 plastic material. Four substances (acetophenone, propanediylbisbenzene, phenyl benzoate and triphenylbenzene) were found in PS (Table 9.1).

Table 9.1. Compounds monitored by GC-MS SIM analysis. The abbreviations or alternative names of the substances, CAS numbers, detection in PS or PTX001 material and known applications is given for each identified chemical.

\begin{tabular}{|c|c|c|c|c|}
\hline Substance abbreviation & CAS number & Full substance name & Detected in & Known uses \\
\hline Acetophenone & $98-86-2$ & Acetophenone & PS, PTX & $\begin{array}{l}\text { Precursor to resins/ } \\
\text { copolymers, used in coatings, } \\
\text { inks and adhesives }\end{array}$ \\
\hline p-Benzoquinone & $719-22-2$ & $\begin{array}{l}\text { 2,5-Cyclohexadiene- } \\
\text { 1,4-dione, 2,6-bis } \\
\text { (1,1-dimethylethyl) }\end{array}$ & PTX & Used in synthesis \\
\hline Dibutylphenol & $96-76-4$ & 2,4-Di-tert-butylphenol & PTX & Antioxidant \\
\hline Propanediylbisbenzene & $1081-75-0$ & $\begin{array}{c}\text { Benzene, } 1,1^{\prime} \text { - } \\
\text { (1,3-propanediyl)bis- }\end{array}$ & PS, PTX & \\
\hline Phenyl benzoate & $93-99-2$ & $\begin{array}{l}\text { Benzoic acid, } \\
\text { phenyl ester }\end{array}$ & PS & $\begin{array}{l}\text { Preservatives used in } \\
\text { cosmetics, film, foods }\end{array}$ \\
\hline TCEP & $115-96-8$ & $\begin{array}{l}\text { Tri(2-chloroethyl) } \\
\text { phosphate }\end{array}$ & PTX & $\begin{array}{l}\text { Plasticizer, flame retardant, } \\
\text { viscosity regulator }\end{array}$ \\
\hline ТСРР (3:1) & $13674-84-5$ & $\begin{array}{l}\text { 2-Propanol, 1-chloro-, } \\
\text { phosphate }(3: 1)\end{array}$ & PTX & Flame retardant \\
\hline \multirow[t]{2}{*}{ ВСРР } & $137888-35-8$ & $\begin{array}{l}\text { Bis(3-chloro-1-propyl) } \\
\text { (1-chloro-2-propyl) } \\
\text { phosphate }\end{array}$ & PTX & \\
\hline & $82304-66-3$ & $\begin{array}{l}\text { 7,9-Di-tert-butyl-1- } \\
\text { oxaspiro(4,5)deca-6,9- } \\
\text { diene-2,8-dione }\end{array}$ & PTX & $\begin{array}{l}\text { Antioxidant } \\
\text { (degradation product) }\end{array}$ \\
\hline DBP & $84-74-2$ & Dibutyl phthalate & PTX & Plasticizer \\
\hline $\mathrm{TPhP}$ & $115-86-6$ & Triphenyl phosphate & PTX & Plasticizer, flame retardant \\
\hline Triphenylbenzene & $28336-57-4$ & $\begin{array}{l}\text { Cyclohexane, } \\
\text { 1,3,5-triphenyl }\end{array}$ & PS, PTX & $\begin{array}{l}\text { Packaging migration residue, } \\
\text { polystyrene impurity }\end{array}$ \\
\hline DEHP & $117-81-7$ & $\begin{array}{l}\text { Bis(2-ethylhexyl) } \\
\text { phthalate }\end{array}$ & PTX & Plasticizer \\
\hline Bumetrizole & 729335 & Bumetrizole & PTX & UV stabilizer \\
\hline $\begin{array}{l}\text { Di(2-ethylhexyl) } \\
\text { terephthalate }\end{array}$ & $6422-86-2$ & $\begin{array}{l}\text { 1,4-Benzenedicarboxylic } \\
\text { acid, bis(2-ethylhexyl) } \\
\text { ester }\end{array}$ & PTX & Plasticizer \\
\hline
\end{tabular}




\section{Replicability of measurements}

Stomach oil extract samples from exposures to PTX001 and PS during the pilot study (0 days, 14 and 90 days) were analysed twice, first using the original pilot study method and secondly using the modified GC-MS analysis method employed in the full study. The results of the two analyses mostly overlap in terms of response, relative to the internal standard (phenanthrene-d10), trend and how it relates to the LOD. In five substances (acetophenone, Propanediylbisbenzene, DBP, DEHP and di(2-ethylhexyl terephthalate), the response scale increased during the re-analysis. It should be acknowledged that the pattern of increase is unidentified (e.g. linear or asymptotic). The relative level of LOD was comparable for all substances during both measurements. The temporal trends indicated were similar for 13 out of 15 substances measured. No good matches were seen for DBP and di(2-ethylhexyl terephthalate. DBP was stable over time during the pilot study, but in re-analysis for the PTX001 sample an increase between 14 and 90 days was observed. For di(2-ethylhexyl terephthalate, the pilot study indicated moderate leaching between 14 and 90 days for both PTX001 and PS, while in the re-analysis this compound remained stable in PS and exhibited a slight decrease in PTX001 samples.

For most samples, the two bottles representing identical treatments (bottle A \& B) show similar averaged results, indicating the reproducibility of the chosen approach. The highest variation between A and B samples was observed in TCPP (3:1) for PTX001 and PS (but not for the controls) during the pilot study and the re-analysis. Another indication for the replicability of the measurements are the control values. Control values remained stable during all measurements with only a few exceptions. Most cases of variation in controls were observed during the first days of the full experiment. The strongest variation was found in DEHP, during full experiment only. The high variation within the first days led to a starting point above PTX001 and PS values. At day 21, the controls stabilized at the same low levels as PTX001 and PS. For TPhP the controls were stable during the pilot study. In the re-analysis, a slight increase above the level of PS was observed at the 90-day measurement.

\section{Additive leaching from plastic to stomach oil}

All experimental results are shown in graphs in the online supplement. Here only part of the results is shown, subdivided in compounds that show leaching, compounds with inconsistent results, and compounds for which no evidence of leaching was observed.

\section{LEACHING OBSERVED}

During the pilot study, leaching of acetophenone (Figure 9.2) was evident for PTX001 after 14 days and decreased slightly at day 90 . However, a similar trend was not observed during the re-analysis of the samples. During the full study, a small degree of leaching from PTX001 was observed until day 21, comparable to the results of the pilot study. Likewise, leaching of acetophenone from PS foam to stomach oil was observed. The leaching of acetophenone from the PS sample was initially rapid during the pilot study, but did not increase substantially between two weeks and three months (Figure 9.2). During 

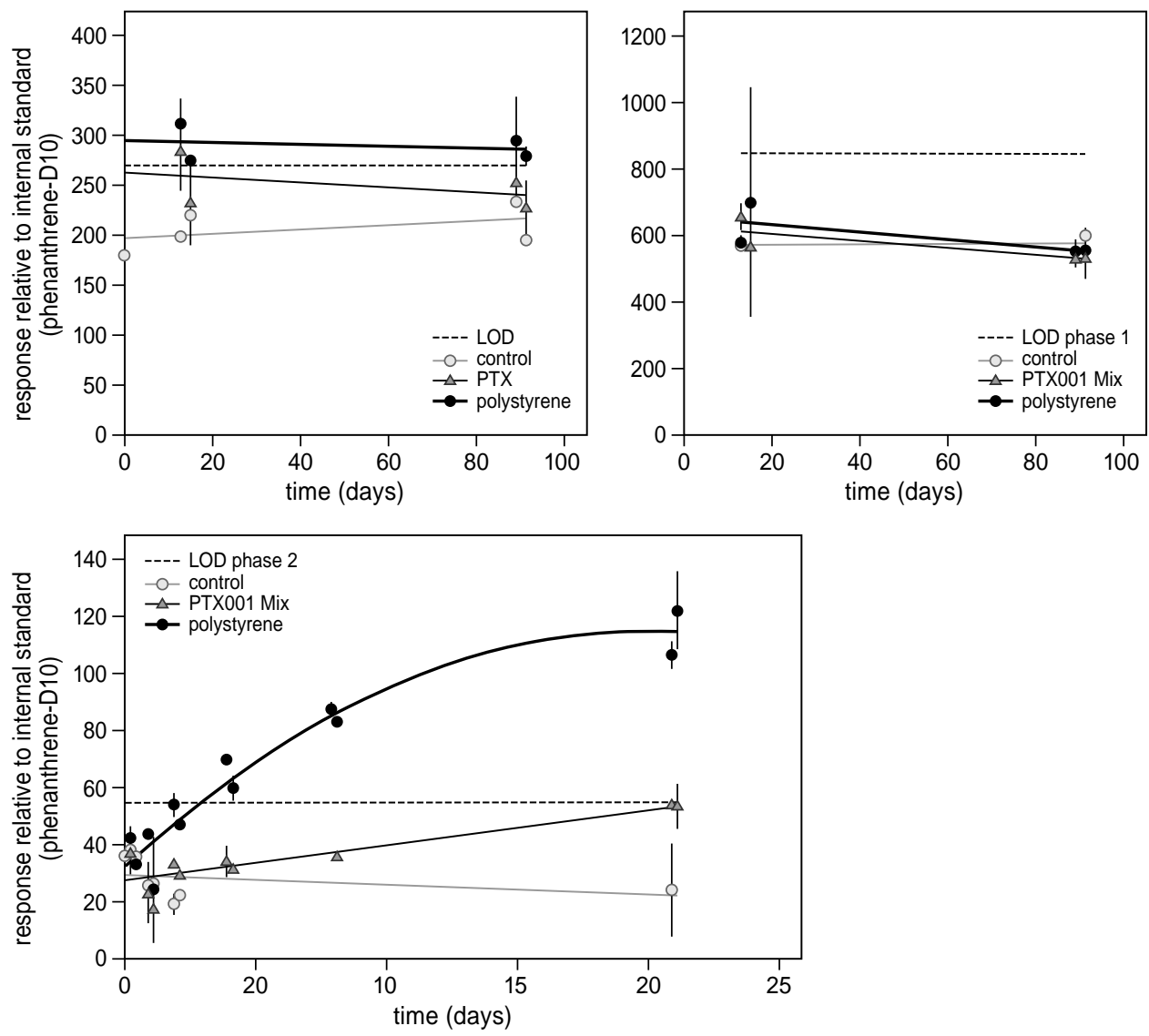

Figure 9.2. Strong leaching of acetophenone from PS and mediate leaching from PTX001 to stomach oil. Top left: Pilot study - long-term exposure. Top right: Re-analysis of the pilot study. Bottom full experiment, short-term exposure. Lines indicate potential changes, but pattern is unidentified, e.g. linear or asymptotic.

the re-analysis, this pattern could not be confirmed, as after a slight increase during the 14-day measurement, the values drop to control level at 90 days. During the full experiment the impression of the pilot study was confirmed, that acetophenone started leaching from PS almost immediately and increased strongly up to day 8, from where on stabilization was observed at day 21 .

For dibutylphenol, strong leaching from PTX001 was clearly shown during all three analyses (Figure 3) and to a lesser extent also in PS. For PTX001, the compound response starts at abundances close to the controls but quickly increases from day 4 until day 21 (final sampling point of the full study). This increase is supported by the pilot study and the re-analysis of the pilot study, where strong increases are indicated in both measurements of the day 90 sample. Although not measured in the original PS sample, dibutylphenol shows higher values than the controls in stomach oil during all three measurements, however values are less pronounced than in PTX001 (Figure 9.3). 

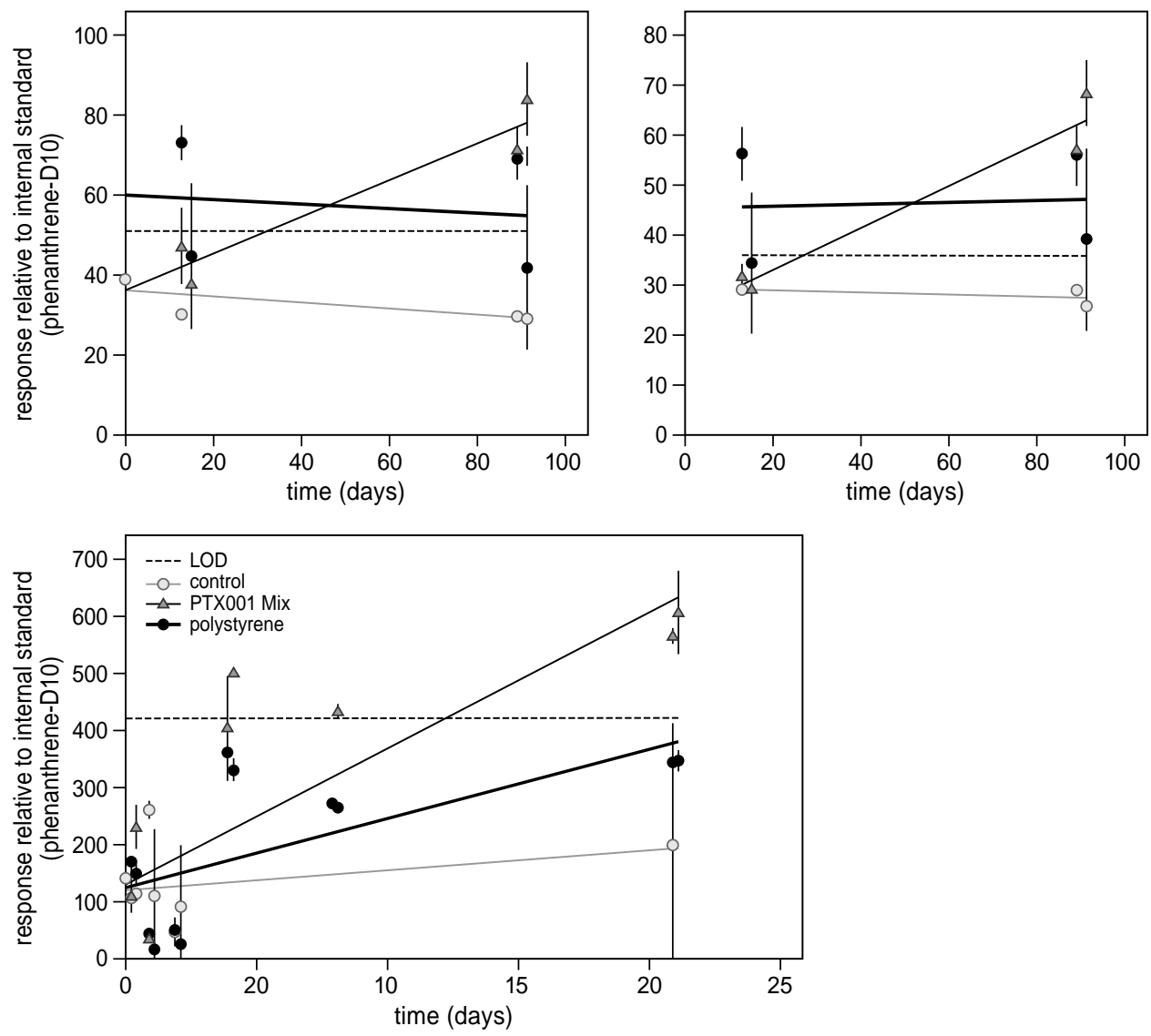

Figure 9.3. Strong leaching of dibutylphenol from PTX001 and moderate leaching from PS to stomach oil. Top left: Pilot study - long-term exposure. Top right: Re-analysis of the pilot study. Bottom full experiment, short-term exposure. Lines indicate potential changes, but pattern is unidentified, e.g. linear or asymptotic.

DEHP shows strong leaching from PTX001 during the pilot study, supported by results from the re-analysis in the full study (Figure 9.4). During the full study, controls show a highly varied pattern over the first two days, indicating less reliable results for this shorterterm exposure. Nevertheless, concentrations of DEHP increase until day 90 in the pilot study. DEHP was not detected in the original PS plastic sample, but was slightly increased during the 14-day measurement of the pilot study and its re-analysis. During the full experiment of PS, DEHP remains under the control level.

For bumetrizole, PTX001 data suggest a high degree of leaching during the first days of the full study (Online Supplement 3.14). The concentration appears to stabilize after 14 days, as shown consistently during the pilot study samples and their re-analysis as part of the full study. Leaching from PTX001 was also observed to a lesser extent for TPhP (Online Supplement 3.11). TPhP leached rapidly into stomach oil during the first 14 days of the pilot study and was confirmed as part of the re-analysis of these samples in the full study. 

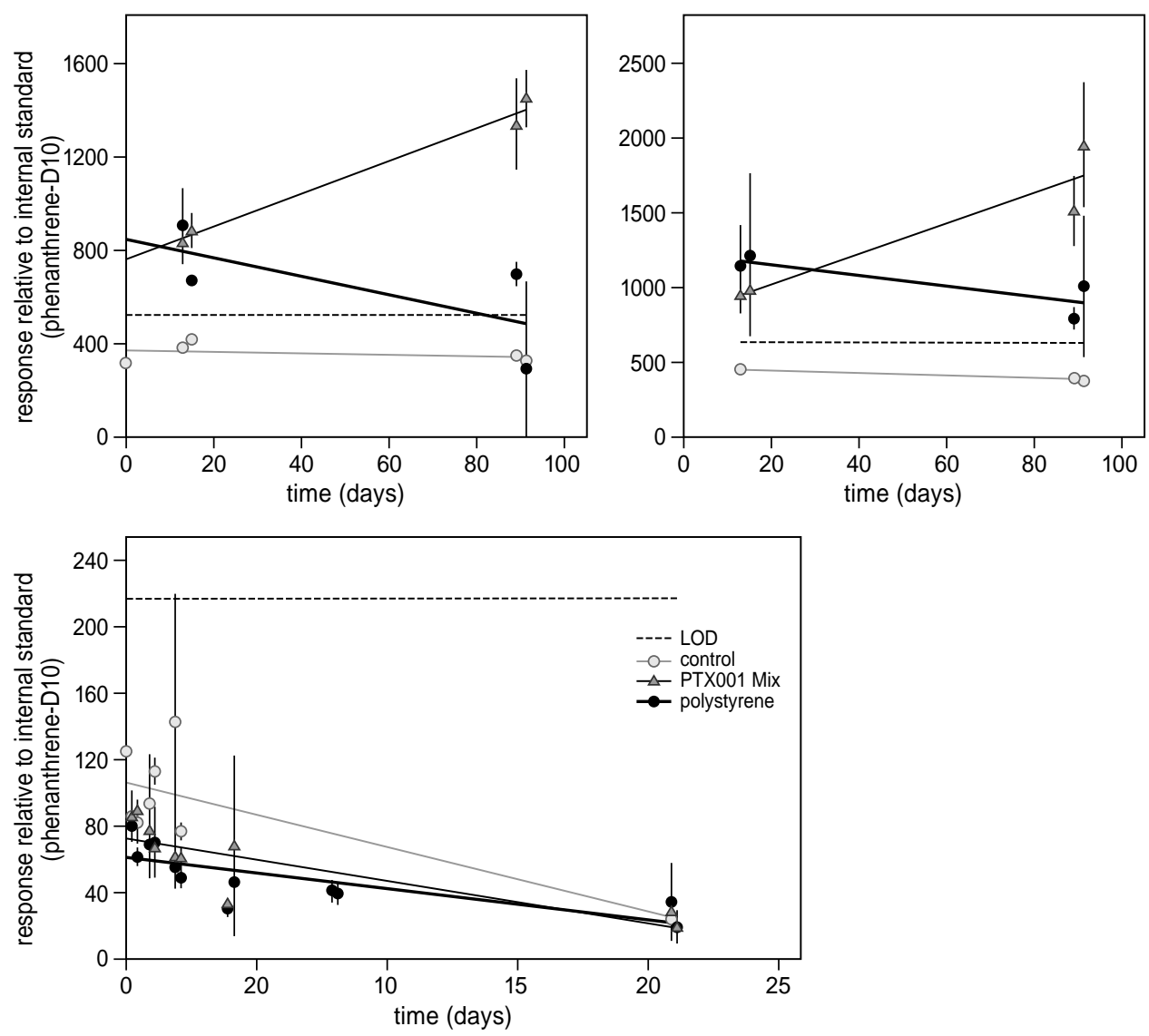

Figure 9.4. Strong leaching of DEHP from PTX001 to stomach oil. For PS no leaching was observed. Top left: Pilot study - long-term exposure. Top right: Re-analysis of the pilot study. Bottom full experiment, short-term exposure. Lines indicate potential changes, but pattern is unidentified, e.g. linear or asymptotic.

$\mathrm{TPhP}$ appeared to stabilize at this level until the last sampling point at day 90. In the full study, however, TPhP did not show any leaching from PTX001 during the first 21 days and it is unclear whether this is a sampling artefact or whether leaching takes place at a later time point.

On a short term, phenyl benzoate (Online Supplement 3.5) showed a strong increase from PS in stomach oil, but long-term data of the pilot study indicate a decrease after two weeks, although at 90 days, the substance is still above the control level. This initial increase, followed by a decrease after two weeks, is confirmed by the re-analysis.

\section{INCONSISTENT LEACHING}

In some substances, the patterns for leaching of substances from PTX001 to stomach oil are inconsistent. For example, the pilot study and the re-analysis of the pilot study samples indicates a high degree of TCEP leaching from PTX001 after 14 days, but this was not 

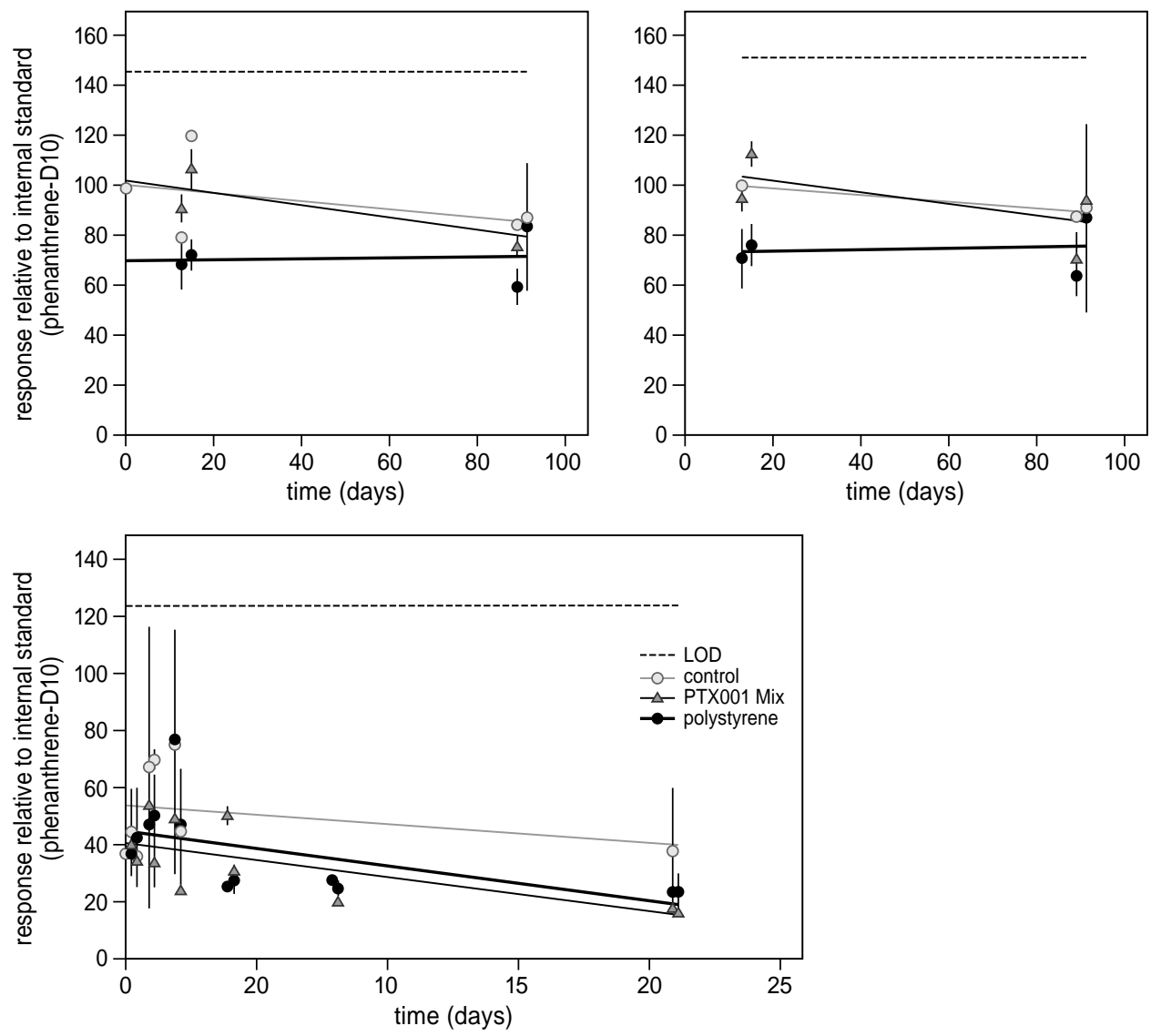

Figure 9.5. No leaching of 7,9-Di-tert-butyl-1-oxaspiro(4,5)deca-6,9-diene-2,8-dione from PTX001 and PS to stomach oil. Top left: Pilot study - long-term exposure. Top right: Re-analysis of the pilot study. Bottom full experiment, short-term exposure. Lines indicate potential changes, but pattern is unidentified, e.g. linear or asymptotic.

observed during the full study, where values are highly variable within measurements and therefore not reliable (Online Supplement 3.6). Phenyl benzoate was not detected in the original PTX001 plastic material and although initial leaching may be suspected from the graphs at day 14 of the pilot study and the re-analysis, all other data points are close to the controls suggesting the presence of this chemical derives from a source other than the PTX001 (Online Supplement 3.5).

\section{NO LEACHING OBSERVED}

No observable leaching from PTX001 was observed for four substances, propanediylbisbenzene, 7,9-Di-tert-butyl-1-oxaspiro(4,5)deca-6,9-diene-2,8-dione, DBP and triphenylbenzene. For PS, propanediylbisbenzene and TPhP, the two other substances recorded in the original PS sample showed no signs of leaching. 
For PTX001, propanediylbisbenzene (Online Supplement 3.4). increases slightly above control levels by the 14-day measurement in the pilot study and remains present in the reanalysis of the samples in the full study. However, concentrations drop to a level below that of the control during the 90-day measurement. During the full study, propanediylbisbenzene remains at a stable level below that of the control samples. 7,9-Di-tert-butyl-1oxaspiro(4,5)deca-6,9-diene-2,8-dione remains close or slightly below control values during all experiments (Figure 9.5). DBP (Online Supplement 3.10) values do not indicate leaching is taking place from PTX001, with only the 90-day measurement of the reanalysis showing a slight increase, but this is not confirmed by other data. Triphenylbenzene (Online Supplement 3.12) present in PTX001, does not show any evidence of leaching into stomach oil in either experiment.

\section{Discussion}

For this study, leaching from marine litter-derived microplastics to the stomach oil of northern fulmars was investigated during exposure experiments. Different types of additives leached from the plastic to the oil. For the microplastic mixture (PTX001), this included precursors to resins and copolymers, antioxidants, plasticizers, flame retardants and UV stabilizers. For PS, leaching of precursors to resins and copolymers, antioxidants and preservatives was observed. More substances leached from PTX001 to the oil as different plastic types (including PS foam) are integrated in this mixture.

This study aimed to investigate leaching of different additives from marine derived plastic litter into the stomach oil of northern fulmars. Difficulties in comparing data from the three experiments may be a result of the novelty of this approach. While measurements of the pilot study and their reanalyses support each other's outcomes in most cases, these results are not directly comparable with the full experiment results, where substantially different responses, relative to the internal standard, have been found. Unfortunately, we do not have a satisfactory answer to that issue. While the pilot study has been analysed separately, the reanalysis was processed together with the full experiment samples, in a randomized order. Both the stomach oil, and the PTX001 and PS samples have the same origin in both experiments. Stomach oil has been stored frozen between the two exposure experiments and no changes in sampling protocol were adapted. Therefore, we have to regard the preliminary study and full experiment, to be separate experiments for shortand long-term exposure. Similarities in the pilot study and its re-analysis, stable controls in most cases and good overlap of A and B samples however, support the reliability of the outcome of this study.

Some of the substances show a higher variation and sometimes unclear patterns during the first days of the full experiment. TCPP (3:1) or BCPP, for example, show varying patterns for all measurements during day $0.3,1$ and 2 . This could be caused by compound instability in the sample matrix, or due to insufficient time for the polymer-oil equilibrium to be achieved. Another possible explanation might be, that due to delays with 
customs, sending samples for the full experiment from the Netherlands to Norway took 16 days. As we cannot exclude the latter option the cause of the high variation, potential delay should be avoided in future.

Substances, such as p-Benzoquinone or phenyl benzoate show an initial increase in stomach oil, but a subsequent decrease at 90 days. Different explanations are possible, all speculative. For example, we should consider vaporization of the substances when exposed to air. Also, metabolisation and biodegradation might occur, when compounds are in contact with oil, resulting in substances, not targeted during our experiment. Finally, it might be speculated that specific compounds tend to gradually attach to the walls of exposure vessels, reducing the concentration in the oil samples over time.

Tanaka et al. (2019) show, that plastics ingested by fulmars and albatrosses contain UV-stabilizers, flame retardants and styrene oligomers, similar to those found in our PTX001 and PS material. Polyethylene and polypropylene were also the most common plastic types encountered. Leaching of substances from plastic to stomach fluids of marine organisms has been observed before by Tanaka et al. (2015). These authors focussed on polybrominated flame retardants and showed, that plastic-related substances can be accumulated, first in stomach oil of short-tailed shearwaters (Puffinus tenuirostris) and then be transferred to the birds' tissue (Tanaka et al. 2013). A direct link from ingested plastics to substances found in bird tissue remains difficult to prove. When studying plastics ingested by fulmars and in a direct comparison with the concentrations of plastic-related substances in tissue, often no direct relationship could be established (Herzke et al. 2016; Provencher et al. 2018; Provencher et al. 2020). But what such studies do, is comparing a 'snapshot of plastic ingestion, with long term accumulation of compounds in tissues. Our results clearly show that several additive substances do leach from plastics to stomach oil and are most likely transferred into the animals' organs together with the oil. It may take some time until these substances are detectable in tissue.

Some of the potential effects these substances may have on fulmars or organisms in general, have been recorded in earlier studies. UV stabilizers are bioaccumulative and are found to be endocrine disruptors and can act mutagenic (Rani et al. 2015). Phthalates, used as plasticizers, have been found to be endocrine disruptive as well and to affect reproduction (Geueke \& Muncke 2017; Oehlmann et al. 2009). DEHP is very common in the environment (Hermabessiere et al. 2017), but our results indicate that it can also leak directly from marine plastic litter to stomach oil of fulmars, offering an additional pathway of uptake. The uptake of DEHP from plastics is enhanced by circumstances found in seabirds' systems, such as high temperatures and low pH values (Bakir et al. 2014). Negative effects of plastic-associated substances on individuals have been observed in experimental studies (Lithner et al. 2009; Hermabessiere et al. 2017). Nevertheless, the sub-lethal effect of these plastic related compounds on the health of populations or species remains difficult to prove (Browne et al. 2015; Werner et al. 2016).

This study cannot contribute to the discussion of pathways of additives either via plastic ingestion or via surrounding water or prey. In our approach we attempted to avoid inclusion of foodweb related chemical pollutants by excluding substances found in the 
untreated stomach oils. The relative importance of both pathways seems difficult to quantify. Studies reporting low uptake of surface sorbed substances (often persistent organic pollutants) via ingested plastic (Bakir et al. 2016) have little relevance for the question of leaching of plastic additives. In addition, these conclusions are based on modelling work or experiments with pristine plastics (Koelmans et al. 2014; Koelmans 2015) that do not properly consider the ingestion of a broad spectrum of highly variable consumer debris items in combination with the unique gastric environment in procellariform seabirds, with high temperatures, low $\mathrm{pH}$ values, the occurrence of stomach oil and the grinding activity in the gizzard. Our results clearly show that ingested plastic can be a vector of several harmful chemical compounds, requiring further work to evaluate actual impacts.

\section{Acknowledgements}

S. Kühn was funded by the Dutch Science Foundation (project ALW-NWO 856.15.001) under EU Joint Program Initiative (JPI) 'Oceans' within the PLASTOX project (Direct and indirect ecotoxicological impacts of microplastics on marine organisms). Albert van Oyen and colleagues helped providing the microplastic mixtures used for the experiments. André Meijboom helped with the execution of the experiments. 


\section{Chapter 10}

\section{Quantitative overview of marine debris}

ingested by marine megafauna

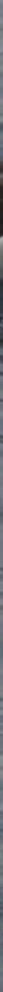




\begin{abstract}
This review quantifies plastic interaction in marine biota. Firstly, entanglement and ingestion records for all marine birds, mammals, turtles, fish, and invertebrate species, are summarized from 747 studies. Marine debris affected 914 species through entanglement and/or ingestion. Ingestion was recorded for 701 species, entanglement was documented for 354 species. Secondly, the individual frequency of occurrence (Ind-\%FO) of ingestion within species was extracted for marine birds, mammals and turtles. Thirdly, for seabird species, average numbers of plastics ingested per individual was determined. Highest Ind-\%FO and average number of plastics was found in tubenosed seabirds with $41 \%$ of all birds analysed having plastics, on average 9.9 particles per bird. The Ind- $\% \mathrm{FO}$ and average number of ingested particles is lower for most other species. However, for certain species, ingestion rates of litter are reason for serious concern. Standardized methods are crucial for future studies, to generate datasets that allow higher level ecosystem analyses.
\end{abstract}




\section{Introduction}

Marine debris and especially plastic litter is a major concern for the general public as well as for scientists and policymakers worldwide. Social-economic costs caused by litter on coastlines and at sea are substantial (Mouat et al. 2010; Newman et al. 2015). But it is the ecological consequences that have created considerable and still growing awareness. Regularly new marine species are encountered to either ingest plastics or to become entangled in it. A first overview of affected species was provided by Laist (1997): the author noted records for either ingestion or entanglement for 267 marine species. In 2015 this list has been expanded to a total of 557 species (Kühn et al. 2015). An even higher number of 693 species was reported by Gall \& Thompson (2015) including organisms that attach on plastics or get smothered by debris. These increases illustrate the research interest in this topic during the last decennia, but not necessarily the increase of affected individuals or species. Most publications have focused on plastic ingestion in seabirds, but recently the number of studies, especially those investigating fish is growing rapidly (Provencher et al. 2017; Markic et al. 2019). Since 2015, several review articles have shown the evergrowing body of literature (e.g. Battisti et al. 2019; Staffieri et al. 2019; Parton et al. 2019), but these were not very successful in extending the simple listing of species to a more in-depth quantitative analysis. Methods applied in studies vary greatly, making comparisons between studies extremely difficult. Nevertheless, an upgrade from a simple species list to a more detailed quantitative overview is crucial for the interpretation of the scale of the impacts of mainly plastic debris on marine wildlife.

This review firstly updates the Kühn et al. (2015) list on records of entanglement in, and ingestion of marine debris. Concerning entanglement records, the distinction between entanglement in active fishing gear and lost or discarded fishing equipment, so-called ghost nets (e.g. Ryan 2018) remains a major problem. For other items such as strap bands, balloon ribbons, plastic bags etc. the classification of marine debris is less ambiguous. Data on entanglement in birds has recently been reviewed by Ryan (2018). Another type of potential risk, closely related to entanglement is the incorporation of plastics in seabird nests. An overview has been recently published by Jagiello et al. (2019) and a website has been launched in 2019 offering the general public to record cases of seabird entanglement or plastic in nests (www.birdsanddebris.com). To study the incidence of entanglement, background data on non-affected individuals in a population are necessary but is usually difficult to obtain. Therefore, the current study focuses on ingestion data but nevertheless provides a list of entangled marine species to allow comparison with earlier work.

The basic information provided by many studies is data on the frequency of occurrence (\%FO) of ingested plastic within a study population. In order to improve on the existing simple species lists, this new review has gathered information on all available data for the $\%$ FO within species of marine birds, mammals and turtles. This allows the creation of species-specific averages of \%FO for plastic ingestion, and derived averages for higher taxa like genera, families or orders. Due to a lack of standardized methods, only a limited number of studies provides data that allows estimates of the average number of plastic 
particles ingested per individual within a species or in higher level taxonomic units. We conducted a number of case studies to highlight the need for more standardization.

\section{Methods}

To be considered for this review, articles and reports had to fulfil certain requirements:

- To be included in this review, reports had to provide the exact species name.

- Only cases of ingestion or entanglement were considered.

- Data on external fouling e.g. in fish gills (e.g. Collard et al. 2017; Abreo et al. 2019), on crustacean carapaces (Welden et al. 2018) etc. were excluded.

- Similarly, impacts on marine sedentary organisms from smothering (e.g. de Carvalho-Souza et al. 2018) have not been included.

- Only studies on animals living in the wild have been included. Experiments where organisms are exposed to plastics were excluded.

- Both dedicated plastic studies and studies where plastic was a side-line, e.g. in traditional diet studies, have been included. While being confident that all publications that dedicatedly reported plastics are included in this review, records of plastics in non-dedicated studies are not always easy to find.

- All, marine debris, including plastics, metal, glass, rubber, natural ropes etc. reported in wildlife were used. However, plastics are by far the most abundant item known to have impacts on marine wildlife and data presented are basically the figures for impact from plastic marine debris.

- All sizes of plastics were included (micro-, meso- and macro plastics).

- Concerning entanglement, we only used data where debris, and not active gear was involved.

- Calculations for ingested plastic include reports of ingested fibres, because in many papers no clear distinction is made between small dust-like fibres (which may partly stem from secondary aerial contamination (Dris et al. 2016; Tanaka \& Takada 2016; Hermsen et al. 2017) and more solid plastic particles.

- Studies published until May 2019 were considered.

\section{Taxonomic \%FO ingestion (Tax-\%FO)}

Firstly, this review provides data on the frequency of occurrence of plastic ingestion and entanglement within higher taxonomic units (Tax-\%FO; e.g. for the number of species within a family). As in Kühn et al. (2015), the Online Supplement (Online Supplement Table 1) contains a detailed table with the underlying information for each species in the taxon. Updates include studies published since the review in 2015 and earlier accounts that had not been detected at that time. In addition two features have been added: dedicated plastic ingestion studies where zero plastics were found in a species, have been included and marked, as recommended by Provencher et al. (2017). Secondly, entangle- 
ment accounts were categorized according to their likelihood of being caused by discarded and lost fishing gear.

Current species classification and names for seabirds were derived from HBW and BirdLife International (2018). The group of marine ducks (Anseriformes) has been expanded in comparison with Kühn et al. (2015), as recommended by Ryan (2018). We've added four species of steamerducks (Tachyeres sp.), three marine mergansers and four other species that spend their time at least partly at sea: long-tailed duck (Clangula hyemalis), black-necked swan (Cygnus melancoryphus), kelp goose (Chloephaga hybrida) and crested duck (Lophonetta specularioides), leading to a total of 26 species of Anseriformes rather than the 13 species considered in Kühn et al. (2015). As the procellariiform and charadriiform orders include many different species, subfamilies were considered. The families of the procellariidae (petrels) and diomedeidae (albatrosses) have been split even further as this group belongs to the most affected family and required more detailed analyses. For marine mammals, names provided by the Society for Marine Mammalogy (2018) were used. All marine bird and mammal species that have been included, are listed in the Online Supplement Table 1. The species numbers for fish and invertebrates were taken from WoRMS (2019). Species numbers differ slightly from previous reviews mainly because of continuous changes in accepted taxonomy. Extinct species and species occurring only in freshwater systems were excluded.

\section{Individual \%FO ingestion (Ind-\%FO)}

Although many species have been recorded with ingested plastics, not all of them are affected to the same extent. One single record for an individual of a species is enough to be included in the Tax-\%FO lists. However, it is the frequency of occurrence within a species that is relevant in terms of potential harm to populations or species. Within species, the individual frequency of occurrence of ingested plastic (Ind-\%FO) could be assessed for marine birds, mammals and turtles. Therefore, all studies that reported the sample size and either the number or the percentage of affected animals are summarized. The studies used are marked with a superscript asterisk in the Online Supplement Reference section. It should be noted that figures may become biased towards the more frequently and better studied species. Fish and invertebrates have been excluded from this detailed overview, as data on $\%$ FO is often incomplete. For fish, available data was recently reviewed by Markic et al. (2019).

Other than the general Tax- $\%$ FO for affected species within a group of species (as done above), the frequency of occurrence within a single species (Ind-\%FO) was calculated as the number of individuals affected, divided by the number of individuals studied per species (group). Studies are included irrespective their methodological setup. In the main paper, results from Ind-\%FO assessed for species, are largely provided as combined data for higher level taxa. This was done by dividing the total number of individuals with ingested plastic, by the total number of individuals studied. Thus, the basis remains the frequency among individuals. Full records for each species are provided in the Online Supplement Table 2. As for many species details of plastic ingestion have not been speci- 
fied, the Ind-\%FO could only be calculated for a limited number of species. Therefore, the percentage of species studied per taxon is provided. For a graphical impression, one species from each taxon was chosen on the basis of having the highest number of dedicated records. When two species had the same number of study records, the one with the higher sample size of individuals has been used.

\section{Quantities of plastics ingested}

Beside the Ind-\%FO, it is the quantity of ingested plastic that is relevant when assessing potential harm to organisms. The interpretation of this data is complex, as there are two common types of reporting average number of plastics in biota:

- the affected average divides the number of items detected by the number of affected organisms.

- the population average is calculated by dividing the number of plastics by all the individuals in the complete sample, thus including the individuals with no plastics.

As already emphasized in Provencher et al. (2017) and Provencher et al. (2019) the population average should be used in order to provide a realistic and comparable overview of the pervasiveness of the plastic problem. For quantities of ingested plastic, this review only used studies where the population average was reported or could be recalculated from the underlying data provided in the papers. Thus, studies were excluded when it was unclear which type of average had been calculated by the authors. All references used for this quantitative part of this study are marked in the Online Supplement References with superscript 2. The quantitative data for individual species were combined into tables for higher taxa by dividing the total number of particles reported by the total number of individuals studied, again including zero records and species with the highest number of study records were depicted.

\section{Results}

A total of 747 studies which reported details on species that either got entangled in or ingested plastic are used for this review. All references are listed in the Online Supplement. The first study included was published in 1938 by Gudger (1938) describing an Atlantic cod (Gadus morhua), entangled in a metal can. There is an older account by the same author from 1931, where a shark has been found entangled in a rubber car tyre (Gudger \& Hoffman 1931), however, as the shark species was not specified, that account could not be included in the literature list. The first account of ingestion of litter by marine organisms comes again from Gudger (1949). In 1931, a tiger shark (Galeocerdo cuvier) was found to have ingested several items of human origin, such as horse shoes, metal cans and rope material. Despite earlier records of ingested marine litter, the first plastic item ingested was found in Leach's storm petrels (Oceanodroma leucorhoa) in 1962 (Rothstein 1973). Since then there has been a continuously growing body of literature (Provencher et al. 2017). 


\section{Taxonomic frequency of occurrence (Tax-\%FO)}

The total number of species encountered either entangled in or with ingested litter has increased from 267 in 1997 (Laist 1997) and 557 in 2015 (Kühn et al. 2015) to currently at least 914 species (Table 10.1). The number of all species and the number of species affected are presented together with the total percentage of affected species. A detailed list of all species with ingestion or entanglement records for marine birds, mammals, turtles, sea snakes, fish and invertebrates is included in Online Supplement Table 1. Although seabirds belong to the species group most intensively studied (Provencher et al. 2017), affected species numbers still increased from 203 in 2015 to 226 in this study. The number of marine mammal species has increased slightly from 81 species in 2015 to 86 species in 2019. In turtles, seven out of seven species were already found to be entangled and to ingest plastics in 2015. The largest increase in studies and species records occurred in fish. In Kühn et al. (2015), 166 fish species had documented cases of plastic entanglement or ingestion. In the current review, this number increased to 430 species of marine fishes. For a full comparison between Kühn et al. (2015) and this study see Online Supplement Table 3.

\section{Individual frequency of occurrence (Ind-\%FO)}

A total of 588 out of 747 studies reports ingestion of plastics by marine wildlife. Eligible for calculations on Ind-\%FO, are 311 studies: 152 for marine birds, 75 for mammals and 84 for turtles. These studies report the sample size and the number or percentage of affected individuals.

Out of 409 known seabird species, 226 species were studied for plastic ingestion of which 180 species have been found with plastics. A total of 43525 individual seabird samples has been studied of which more than a quarter (12065 individuals, 27.7\%) contain plastics. Procellariiformes are among the best studied taxon with 103 out of 144 species and 22735 individuals analysed. This group exhibits the highest Ind- \%FO with $41.5 \%$ of all individuals containing plastic (Table 10.2).

While $41.5 \%$ of the marine mammal species has currently been studied for plastic ingestion, only 860 out of 19486 individuals (4\%) contained plastics (Table 10.3). For both, baleen and toothed whales, around half of the species have been studied. Baleen whales showed a higher Ind-\%FO (16.67\%) than toothed whales (9.4\%). However only 96 baleen but 5002 toothed whales have been autopsied. Plastic has been found in the digestive tracts of seals but not in polar bears or otters, the Ind-\%FO for carnivores remains low at $0.95 \%$.

There are only seven species of marine turtles and all of them, with exception of the flatback turtle (Natator depressus), have been repeatedly encountered with ingested plastics. Therefore, results are presented in a table for each species separately (Table 10.4). In contrast to the small number of species, plastic in turtles gained a lot of attention in scientific literature, with 140 study records. One third (32\%) of 7879 turtles analysed contained plastic in their stomachs. 
Table 10.1. Overview plastic ingestion or entanglement or both in the main animal taxa. Shown are the total number of species within the taxon, the number of species known to be affected, and the derived percentage of affected species within the taxon (Tax-\%FO). Species specific data underlying the taxon figures are given in Online Supplement Table 1).

\begin{tabular}{|c|c|c|c|c|c|c|c|}
\hline \multirow[b]{2}{*}{ Taxa } & \multirow[b]{2}{*}{$\begin{array}{r}\mathrm{n} \text { species } \\
\text { in taxon }\end{array}$} & \multicolumn{2}{|c|}{ Ingestion } & \multicolumn{2}{|c|}{ Entanglement } & \multicolumn{2}{|c|}{$\begin{array}{c}\text { Ingestion \& } \\
\text { Entanglement }\end{array}$} \\
\hline & & $\begin{array}{r}n \\
\text { affected } \\
\text { species }\end{array}$ & $\begin{array}{r}\text { Tax- } \\
\% \text { FO }\end{array}$ & $\begin{array}{r}n \\
\text { affected } \\
\text { species }\end{array}$ & $\begin{array}{l}\text { Tax- } \\
\% \mathrm{FO}\end{array}$ & $\begin{array}{r}n \\
\text { affected } \\
\text { species }\end{array}$ & $\begin{array}{l}\text { Tax- } \\
\% \text { FO }\end{array}$ \\
\hline \multicolumn{8}{|l|}{ Seabirds } \\
\hline Anseriformes (marine ducks) & 26 & 2 & $7.7 \%$ & 6 & $23.1 \%$ & 6 & $23.1 \%$ \\
\hline Podicipediformes (grebes) & 20 & 0 & $0.0 \%$ & 6 & $30.0 \%$ & 6 & $30.0 \%$ \\
\hline Phaetontiformes (tropicbirds) & 3 & 2 & $66.7 \%$ & 1 & $33.3 \%$ & 3 & $100.0 \%$ \\
\hline Gaviformes (loons) & 5 & 4 & $80.0 \%$ & 5 & $100.0 \%$ & 5 & $100.0 \%$ \\
\hline Sphenisciformes (penguins) & 18 & 5 & $27.8 \%$ & 6 & $33.3 \%$ & 9 & $50.0 \%$ \\
\hline Procellariiformes (tubenoses) & 144 & 91 & $63.2 \%$ & 18 & $12.5 \%$ & 91 & $63.2 \%$ \\
\hline Pelecaniformes (pelicans) & 8 & 3 & $37.5 \%$ & 4 & $50.0 \%$ & 5 & $62.5 \%$ \\
\hline Suliformes (gannets, cormorants) & 49 & 15 & $30.6 \%$ & 19 & $38.8 \%$ & 24 & $49.0 \%$ \\
\hline $\begin{array}{l}\text { Charadriiformes } \\
\text { (gulls, terns, skuas, auks) }\end{array}$ & 136 & 58 & $42.6 \%$ & 47 & $34.6 \%$ & 77 & $56.6 \%$ \\
\hline All seabirds & 409 & 180 & $44.0 \%$ & 112 & $27.4 \%$ & 226 & $55.3 \%$ \\
\hline \multicolumn{8}{|l|}{ Marine mammals } \\
\hline Ursidae (polar bears) & 1 & 0 & $0.0 \%$ & 1 & $100.0 \%$ & 1 & $100.0 \%$ \\
\hline Mustelidae (marine otters) & 2 & 0 & $0.0 \%$ & 2 & $100.0 \%$ & 2 & $100.0 \%$ \\
\hline Pinnipedia (all seals) & 31 & 15 & $48.4 \%$ & 22 & $71.0 \%$ & 22 & $71.0 \%$ \\
\hline Otariidae (eared seals) & 14 & 10 & $71.4 \%$ & 12 & $85.7 \%$ & 12 & $85.7 \%$ \\
\hline Odobenidae (walruses) & 1 & 0 & $0.0 \%$ & 0 & $0.0 \%$ & 0 & $0.0 \%$ \\
\hline Phocidae (true seals) & 16 & 5 & $31.3 \%$ & 10 & $62.5 \%$ & 10 & $62.5 \%$ \\
\hline Cetartiodactyla (all whales) & 86 & 52 & $60.5 \%$ & 22 & $25.6 \%$ & 59 & $68.6 \%$ \\
\hline Mysticeti (baleen whales) & 14 & 8 & $57.1 \%$ & 10 & $71.4 \%$ & 12 & $85.7 \%$ \\
\hline Odontoceti (toothed whales) & 72 & 44 & $61.1 \%$ & 12 & $16.7 \%$ & 47 & $65.3 \%$ \\
\hline Sirenia (manatees, dugongs) & 3 & 2 & $66.7 \%$ & 2 & $66.7 \%$ & 2 & $66.7 \%$ \\
\hline All marine mammals & 123 & 69 & $56.1 \%$ & 49 & $39.8 \%$ & 86 & $69.9 \%$ \\
\hline \multicolumn{8}{|l|}{ Other taxa } \\
\hline All turtles & 7 & 7 & $100.0 \%$ & 7 & $100.0 \%$ & 7 & $100.0 \%$ \\
\hline All sea snakes & 62 & 0 & $0.0 \%$ & 2 & $3.2 \%$ & 2 & $3.2 \%$ \\
\hline All fish & 31243 & 363 & & 101 & & 430 & \\
\hline All invertebrates & 159000 & 82 & & 83 & & 163 & \\
\hline All species & & 701 & & 354 & & 914 & \\
\hline
\end{tabular}


Table 10.2. Individual frequency of occurrence (Ind-\%FO) of plastic ingestion for seabirds per taxon. The total number of species in the taxon is given with the percentage of species within the taxon for which ingestion studies are available. For each taxon, the number of individuals studied, and the number of individuals with plastic is tabulated, with the derived Ind-\%FO shown in the last column.

\begin{tabular}{|c|c|c|c|c|c|}
\hline Taxon & n species & $\begin{array}{r}\% \text { species } \\
\text { studied }\end{array}$ & $\begin{array}{r}\text { Number of } \\
\text { individuals } \\
\text { studied }\end{array}$ & $\begin{array}{l}\text { Number of } \\
\text { individuals } \\
\text { with plastic }\end{array}$ & Ind-FO $\%$ \\
\hline Anseriformes & 26 & $38.5 \%$ & 823 & 2 & $0.2 \%$ \\
\hline Podicipediformes & 20 & $15.0 \%$ & 8 & 0 & $0.0 \%$ \\
\hline Phaetontiformes & 3 & $100.0 \%$ & 221 & 31 & $14.0 \%$ \\
\hline Gaviformes & 5 & $80.0 \%$ & 32 & 3 & $9.4 \%$ \\
\hline Sphenisciformes & 18 & $38.9 \%$ & 1478 & 214 & $14.5 \%$ \\
\hline \multicolumn{6}{|l|}{ Procellariiformes } \\
\hline Storm petrels & 27 & $48.1 \%$ & 1614 & 415 & $25.7 \%$ \\
\hline Great albatrosses & 6 & $83.3 \%$ & 157 & 29 & $18.5 \%$ \\
\hline N Pacific albatrosses & 4 & $100.0 \%$ & 1434 & 1205 & $84.0 \%$ \\
\hline Sooty albatrosses & 2 & $100.0 \%$ & 83 & 2 & $2.4 \%$ \\
\hline Mollymawks & 10 & $100.0 \%$ & 2301 & 114 & $5.0 \%$ \\
\hline Fulmarine petrels & 7 & $100.0 \%$ & 4300 & 2725 & $63.4 \%$ \\
\hline Whalebirds & 8 & $75.0 \%$ & 819 & 584 & $71.3 \%$ \\
\hline Gadfly petrels & 35 & $68.6 \%$ & 1420 & 233 & $16.4 \%$ \\
\hline Procellaria petrels & 5 & $100.0 \%$ & 875 & 92 & $10.5 \%$ \\
\hline Shearwaters & 30 & $60.0 \%$ & 7258 & 4000 & $55.1 \%$ \\
\hline Bulweria petrels & 6 & $50.0 \%$ & 289 & 5 & $1.7 \%$ \\
\hline Diving petrels & 4 & $75.0 \%$ & 313 & 21 & $6.7 \%$ \\
\hline All Procellariiformes & 144 & $71.5 \%$ & 22735 & 9426 & $41.5 \%$ \\
\hline Pelecaniformes & 8 & $37.5 \%$ & 31 & 12 & $38.7 \%$ \\
\hline \multicolumn{6}{|l|}{ Suliformes } \\
\hline Frigatebirds & 5 & $60.0 \%$ & 96 & 23 & $24.0 \%$ \\
\hline Gannets & 10 & $60.0 \%$ & 984 & 262 & $26.6 \%$ \\
\hline Cormorants & 34 & $26.5 \%$ & 580 & 51 & $8.8 \%$ \\
\hline All Suliformes & 49 & $36.7 \%$ & 1660 & 336 & $20.2 \%$ \\
\hline \multicolumn{6}{|l|}{ Charadriiformes } \\
\hline Sheathbills & 2 & $100.0 \%$ & 5 & 3 & $60.0 \%$ \\
\hline Phalaropes & 3 & $66.7 \%$ & 113 & 65 & $57.5 \%$ \\
\hline Noddies & 5 & $80.0 \%$ & 258 & 15 & $5.8 \%$ \\
\hline White terns & 2 & $50.0 \%$ & 54 & 1 & $1.9 \%$ \\
\hline Skimmers & 3 & $0.0 \%$ & 0 & 0 & $0.0 \%$ \\
\hline Gulls & 51 & $49.0 \%$ & 4840 & 788 & $16.3 \%$ \\
\hline Terns & 39 & $51.3 \%$ & 1313 & 21 & $1.6 \%$ \\
\hline Skuas & 7 & $85.7 \%$ & 2192 & 180 & $8.2 \%$ \\
\hline Auks & 24 & $79.2 \%$ & 16537 & 2041 & $12.3 \%$ \\
\hline All Charadriiformes & 136 & $\mathbf{5 5 . 9} \%$ & 16537 & 2041 & $12.3 \%$ \\
\hline All se & 409 & $55.3 \%$ & 43525 & 12065 & $27.7 \%$ \\
\hline
\end{tabular}


Table 10.3. Individual frequency of occurrence (Ind-\%FO) of plastic ingestion for marine mammals per taxon. The total number of species in the taxon is given with the percentage of species within the taxon for which ingestion studies are available. For each taxon, the number of individuals studied, and the number of individuals with plastic is tabulated, with the derived Ind-\%FO shown in the last column.

\begin{tabular}{|c|c|c|c|c|c|}
\hline Taxon & n species & $\begin{array}{r}\% \text { species } \\
\text { studied }\end{array}$ & $\begin{array}{r}\text { Number of } \\
\text { individuals } \\
\text { studied }\end{array}$ & $\begin{array}{l}\text { Number of } \\
\text { individuals } \\
\text { with plastic }\end{array}$ & Ind-FO $\%$ \\
\hline \multicolumn{6}{|l|}{ Carnivores } \\
\hline Ursidae & 1 & $0.00 \%$ & 0 & 0 & $0.00 \%$ \\
\hline Mustelidae & 3 & $0.00 \%$ & 0 & 0 & $0.00 \%$ \\
\hline Otariidae & 14 & $28.57 \%$ & 8593 & 58 & $0.67 \%$ \\
\hline Odobenidae & 1 & $0.00 \%$ & 0 & 0 & $0.00 \%$ \\
\hline Phocidae & 16 & $25.00 \%$ & 1191 & 34 & $2.85 \%$ \\
\hline All carnivores & 34 & $23.53 \%$ & 9784 & 93 & $0.95 \%$ \\
\hline \multicolumn{6}{|l|}{ Baleen whales } \\
\hline Balaenidae & 4 & $0.00 \%$ & 0 & 0 & $0.00 \%$ \\
\hline Neobalaenidae & 1 & $0.00 \%$ & 0 & 0 & $0.00 \%$ \\
\hline Eschritiidae & 1 & $0.00 \%$ & 0 & 0 & $0.00 \%$ \\
\hline Balaenopteridae & 8 & $75.00 \%$ & 96 & 16 & $16.67 \%$ \\
\hline All baleen whales & 14 & $42.86 \%$ & 96 & 16 & $16.67 \%$ \\
\hline \multicolumn{6}{|l|}{ Toothed whales } \\
\hline Physteridae & 1 & $100.00 \%$ & 145 & 37 & $25.52 \%$ \\
\hline Kogiidae & 2 & $100.00 \%$ & 39 & 10 & $25.64 \%$ \\
\hline Ziphiidae & 22 & $54.55 \%$ & 266 & 66 & $24.81 \%$ \\
\hline Pontoporiidae & 1 & $100.00 \%$ & 197 & 44 & $22.34 \%$ \\
\hline Monodontidae & 2 & $50.00 \%$ & 1 & 1 & $100.00 \%$ \\
\hline Delphinidae & 37 & $45.95 \%$ & 2495 & 205 & $8.22 \%$ \\
\hline Phocoenidae & 7 & $28.57 \%$ & 1859 & 107 & $5.76 \%$ \\
\hline All toothed whales & 72 & $50.00 \%$ & 5002 & 470 & $9.40 \%$ \\
\hline All cetaceans & 86 & $48.84 \%$ & 5098 & 486 & $9.53 \%$ \\
\hline \multicolumn{6}{|l|}{ Sirenia } \\
\hline Trichechidae & 2 & $50.00 \%$ & 4604 & 281 & $6.10 \%$ \\
\hline Dugongidae & 1 & $0.00 \%$ & 0 & 0 & $0.00 \%$ \\
\hline All sirenia & 3 & $33.33 \%$ & 4604 & 281 & $6.10 \%$ \\
\hline All marine $\mathrm{m}$ & 123 & $41.46 \%$ & 19486 & 860 & $4.41 \%$ \\
\hline
\end{tabular}

Figure 10.1 provides the Ind-\%FO for selected species having the highest number of dedicated study records within the higher level taxon. Thus, please note that also speciesspecific data are often derived from many different studies. The supplementary Table 2 provides data for all species studied. Low sample numbers can lead to unusual high \%FO's of e.g. 100\% (e.g. black-faced sheathbill (Chionis minor), narwhal (Monodon monoceros) and flatback turtle). High Ind-\%FO's with sufficient sample numbers are mainly reached in seabirds. Laysan albatross (Phoebastria immutabilis), northern fulmar (Fulmarus glacialis), 
Table 10.4. Individual frequency of occurrence (Ind-\%FO) of plastic ingestion for turtles. The species is given with the percentage of species within the taxon for which ingestion studies are available. For each taxon, the number of individual studied, and the number of individuals with plastic is tabulated, with the derived Ind-\%FO shown in the last column.

\begin{tabular}{|c|c|c|c|c|c|}
\hline Taxon & n species & $\begin{array}{r}\% \text { species } \\
\text { studied }\end{array}$ & $\begin{array}{r}\text { Number of } \\
\text { individuals } \\
\text { studied }\end{array}$ & $\begin{array}{l}\text { Number of } \\
\text { individuals } \\
\text { with plastic }\end{array}$ & Ind-FO $\%$ \\
\hline Loggerhead turtle & 1 & $100 \%$ & 3919 & 843 & $22 \%$ \\
\hline Kemp's ridley turtle & 1 & $100 \%$ & 304 & 106 & $35 \%$ \\
\hline Olive ridley turtle & 1 & $100 \%$ & 179 & 81 & $45 \%$ \\
\hline Green turtle & 1 & $100 \%$ & 2720 & 1275 & $47 \%$ \\
\hline Hawksbill turtle & 1 & $100 \%$ & 86 & 31 & $36 \%$ \\
\hline Flatback turtle & 1 & $100 \%$ & 2 & 2 & $100 \%$ \\
\hline Leatherback turtle & 1 & $100 \%$ & 669 & 198 & $30 \%$ \\
\hline & 7 & $100 \%$ & 7879 & 2536 & $32 \%$ \\
\hline
\end{tabular}

sooty shearwater (Ardenna grisea) and red phalarope (Phalaropus fulicarius) are species with more than $50 \%$ of the analysed individuals containing plastics. A high Ind-\%FO in marine mammals was found in sperm whales (Physeter macrocephalus; 26\%) and green turtles (Chelonia mydas; 47\%).

\section{Average number of plastic items in seabirds}

Less than a third of all study records of marine mammals and turtles report population averages for the number of rubbish or plastic items (27.9\% of 218 and $31.2 \%$ of 189 study records, respectively). Consequently, we focus on seabirds, where almost half of the studies (48.9\% of 841 study records, reported in 80 studies) provide population averages on numbers of plastics ingested.

Table 10.5 provides data on the average number of plastic items per individual within seabird taxa. In 7572 procellariform birds, on average 9.88 pieces of plastics were encountered, almost ten times as many plastic pieces as in Charadriiformes. More than the half (53\%) of all charadriiform species have records of average numbers and 9284 individuals have been analysed. Calculated for all these individuals, on average they have ingested 1.03 plastic pieces. Phalaropes and auks contain on average more than one piece of plastic ( 8 and 1.6 respectively), while terns have only 0.006 pieces of plastic on average, despite the relatively large sample size of 325 birds. In all other bird orders sample sizes were much smaller and these birds contained on average less than one particle per bird. The maximum average number reported in a species group concerns the smallest member of the procellariform seabird, the storm petrels. Youngren et al. (2018) report an average of 203 plastic pieces in Tristram's storm petrels (Oceanodroma tristrami) on Hawaii with a maximum of 615 plastic pieces in a single bird. The second highest record was also found on Hawaii, in Laysan albatross fledglings. Lavers \& Bond (2016) found an average number 

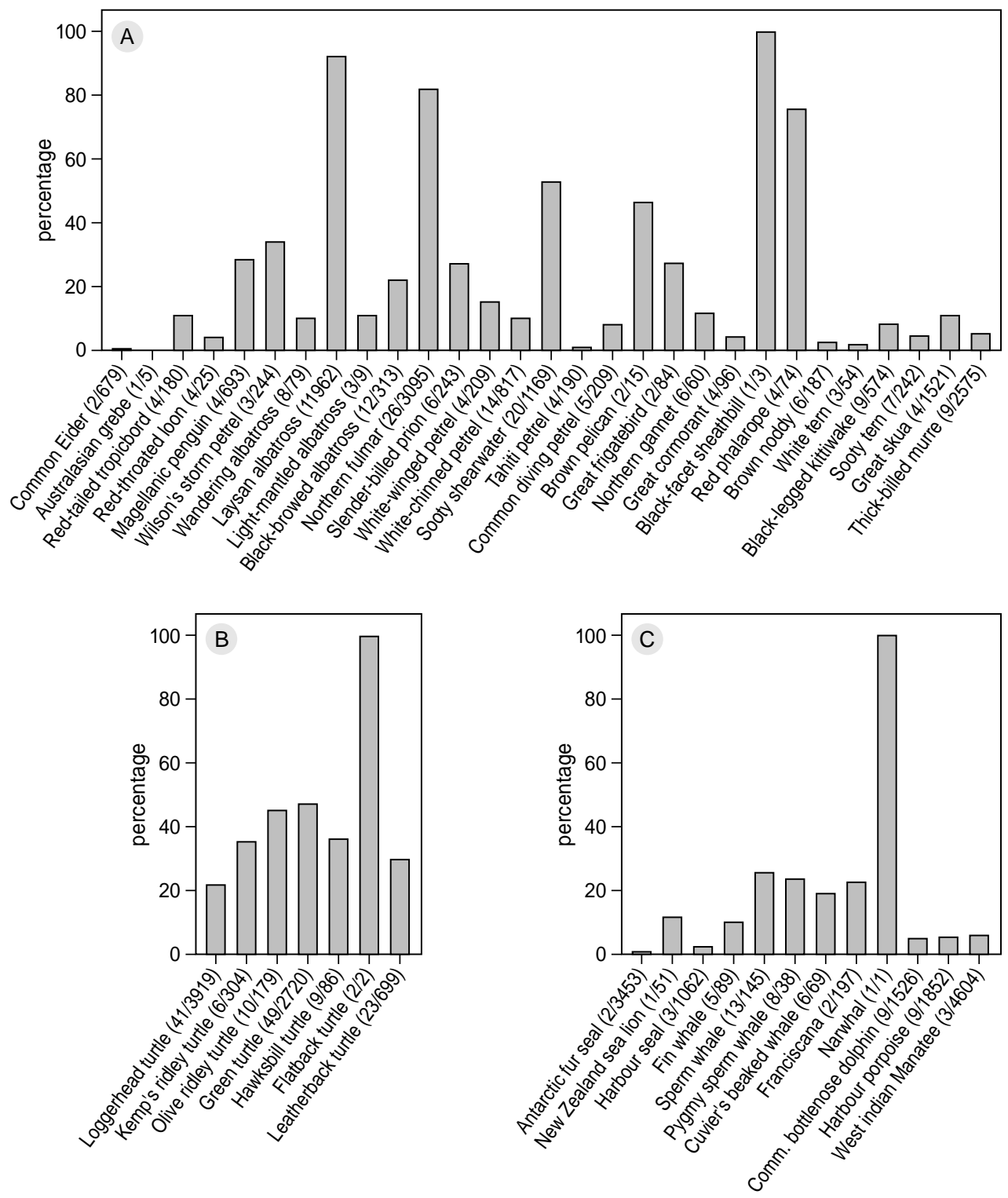

Figure 10.1. Individual frequency of occurrence (Ind-\%FO) of ingested plastics for a selection of best documented species of seabirds (A), turtles (B) and marine mammals (C). Species shown here were chosen from their taxonomic group as the species with the highest number of study records. With the species names, the number of studies on that species and the number of individuals used for the Ind-\%FO calculations are shown in parenthesis.

of 132.5 pieces and a maximum of 450 pieces in one bird. Other members of the procellariform order such as bulweria petrels, diving petrels and mollymawks contained $<0.017$ plastic particles on average. 
Table 10.5. Average number of plastic pieces ingested per individual for the major seabird taxa. The total number of species in the taxon is given with the percentage of species within the taxon for which ingestion studies are available. For each taxon, the number of individual studied, and the population average of plastic items per bird is tabulated. The range of the average number of plastics per bird is shown for all studies considered for this calculation.

\begin{tabular}{|c|c|c|c|c|c|c|}
\hline \multirow[t]{2}{*}{ Taxon } & \multirow[t]{2}{*}{ n species } & \multirow[t]{2}{*}{$\begin{array}{r}\% \text { species } \\
\text { studied }\end{array}$} & \multirow{2}{*}{$\begin{array}{r}\text { Number of } \\
\text { individuals } \\
\text { studied }\end{array}$} & \multirow{2}{*}{$\begin{array}{r}\text { Average } \\
\text { number } \\
\text { plastics/bird }\end{array}$} & \multicolumn{2}{|c|}{$\begin{array}{l}\text { Reported rane } \\
\text { average in studies }\end{array}$} \\
\hline & & & & & $\min$ & $\max$ \\
\hline Anseriformes & 26 & $38 \%$ & 864 & 0.012 & 0 & 0.175 \\
\hline Podicipediformes & 20 & $0 \%$ & 8 & 0 & 0 & 0 \\
\hline Phaetontiformes & 3 & $100 \%$ & 15 & 0.067 & 0 & 0.3 \\
\hline Gaviformes & 5 & $80 \%$ & 14 & 0.071 & 0 & 0.333 \\
\hline Sphenisciformes & 18 & $22 \%$ & 143 & 0.014 & 0 & 0.222 \\
\hline \multicolumn{7}{|l|}{ Procellariiformes } \\
\hline Storm-petrels & 27 & $41 \%$ & 939 & 13.122 & 0 & 203.2 \\
\hline Great albatrosses & 6 & $67 \%$ & 88 & 0.42 & 0 & 1 \\
\hline N. Pac. albatrosses & 4 & $75 \%$ & 154 & 36.935 & 0 & 132.46 \\
\hline Sooty albatrosses & 2 & $0 \%$ & 3 & 0 & 0 & 0 \\
\hline Mollymawks & 10 & $60 \%$ & 765 & 0.012 & 0 & 1.5 \\
\hline Fulmarine petrels & 7 & $86 \%$ & 2437 & 17.795 & 0 & 65.4 \\
\hline Whalebirds & 8 & $50 \%$ & 84 & 1.048 & 0 & 5.5 \\
\hline Gadfly petrels & 35 & $43 \%$ & 529 & 0.476 & 0 & 4.109 \\
\hline Procellaria petrels & 5 & $40 \%$ & 126 & 8.127 & 0 & 8.947 \\
\hline Shearwaters & 30 & $53 \%$ & 2207 & 5.454 & 0 & 43 \\
\hline Bulweria petrels & 6 & $33 \%$ & 182 & 0.005 & 0 & 0.008 \\
\hline Diving petrels & 4 & $25 \%$ & 58 & 0.017 & 0 & 0.02 \\
\hline All Procellariiformes & 144 & $49 \%$ & 7572 & 9.882 & 0 & 203.2 \\
\hline Pelecaniformes & 8 & $13 \%$ & 15 & 0.53 & 0.53 & 0.53 \\
\hline \multicolumn{7}{|l|}{ Suliformes } \\
\hline Frigatebirds & 5 & $40 \%$ & 12 & 0 & 0 & 0 \\
\hline Gannets & 10 & $50 \%$ & 92 & 1.457 & 0 & 5.81 \\
\hline Cormorants & 34 & $24 \%$ & 207 & 0.415 & 0 & 2 \\
\hline All Suliformes & 49 & $31 \%$ & 311 & 0.704 & 0 & 5.81 \\
\hline \multicolumn{7}{|l|}{ Charadriiformes } \\
\hline Sheathbills & 2 & $50 \%$ & 2 & 0 & 0 & 0 \\
\hline Phalaropes & 3 & $67 \%$ & 22 & 8 & 1 & 12.3 \\
\hline Noddies & 5 & $80 \%$ & 22 & 0 & 0 & 0 \\
\hline White terns & 2 & $50 \%$ & 54 & 0.093 & 0 & 0.625 \\
\hline Skimmers & 3 & $0 \%$ & 0 & na & na & na \\
\hline Gulls & 51 & $43 \%$ & 2827 & 0.736 & 0 & 9.8 \\
\hline Terns & 39 & $46 \%$ & 328 & 0.006 & 0 & 0.031 \\
\hline Skuas & 7 & $71 \%$ & 1465 & 0.145 & 0 & 30 \\
\hline Auks & 24 & $79 \%$ & 4564 & 1.559 & 0 & 47 \\
\hline All Charadriiformes & 136 & $53 \%$ & 9284 & 1.033 & 0 & 47 \\
\hline All seabirds & 409 & $44 \%$ & 25798 & 3.282 & 0 & 203 \\
\hline
\end{tabular}


Again, as for Ind-\%FO, a selection of species with the highest study record per taxon group was made to illustrate data on the average number of ingested plastic items. Laysan albatross, northern fulmar, white-chinned petrel (Procellaria aequinoctialis), short-tailed shearwater (Ardenna tenuirostris), northern gannet (Morus bassanus) and red phalarope exceed the average of more than one piece of ingested plastic per individual. To visualize this data, two graphs were made for species with either an average of $<1$ or $>1$ pieces, respectively (Figure 10.2).

\section{Discussion}

This review, presents the most recent updated information on documented cases of plastic ingestion and entanglement in marine species. In recent years many reviews have been published focusing on either entanglement or ingestion and different taxon groups such as seabirds (Ryan 2018; Battisti et al. 2019; Jagiello et al. 2019), marine mammals (Fossi et al. 2018; Stelfox \& Hudgins 2015), turtles (Staffieri et al. 2019), fish (Azevedo-Santos et al. 2019; Markic et al. 2019; Parton et al. 2019) and zooplankton (Botterell et al. 2019). The number of affected species in these studies vary, as different criteria for species selection were applied. Battisti et al. (2019) and Azevedo-Santos et al. (2019) for example, included terrestrial bird species, but Battisti et al. (2019) also included many seabird species clearly caught in active fishing gear, confounding two very relevant but distinctive threats to seabirds.

The current study combines data on all marine taxa and presents a comprehensive list of all marine species recorded with plastic entanglement or ingestion. This list should serve as a useful tool for e.g. scientists to quickly gain insights in what is known about plastic ingestion or entanglement in a specific species, updating the list by Kühn et al. (2015). Beside this tool, this study provides insight in the number of affected individuals within species of marine megafauna and an overview of quantities of plastics found in seabirds. Unfortunately, it was not possible to provide an overview of data on average plastic mass, not even in the well-studied seabird group. Beside many zero-accounts, only 38 studies report population averages of plastic mass in seabirds, insufficient to compile a reliable overview for different species groups. Long-term studies in northern fulmars in the Netherlands show that the average mass of plastic particles decreased since the 1980s, indicating a trend towards more, but smaller plastics (Van Franeker et al. 2011; Van Franeker \& Law 2015). Plastic mass or volume would be a better indicator of impact on animals ingesting plastic than the number of plastic particles. Therefore, for long-term data collection, plastic mass should be considered the most reliable unit and should be provided as population average when studying plastics in marine organisms (Provencher et al. 2019).

Our numbers show, that plastics occur in many species, living in different marine habitats around the world, feeding and digesting in different ways. Interaction with plastic has been reported in tiny barnacles (Goldstein \& Goodwin 2013) and in blue whales (Baxter 2009). It has been reported in different species from remote places such as polar regions 

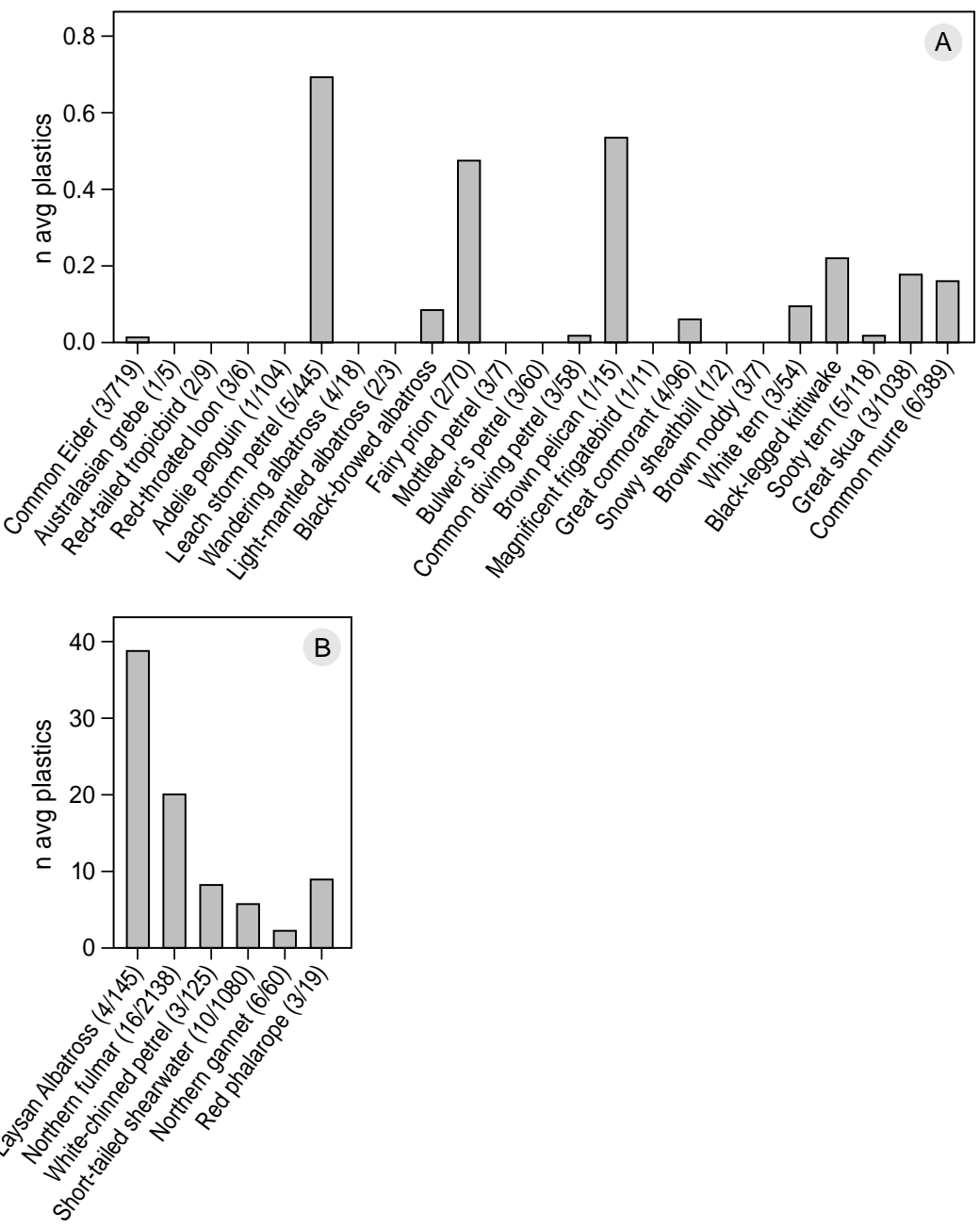

Figure 10.2. Population average number of ingested plastic particles in seabirds. Species shown here were chosen from their taxonomic group as the species with the highest number of study records with the species names. The number of studies on that species and the number of individuals used are shown in parenthesis. (A) The graph shows seabird species with on average $<1$ piece of plastic per bird. (B) The graph shows seabird species with an average of $>1$ plastic items.

(Nielsen et al. 2013; Poon et al. 2016; Ainley et al. 1990a; Van Franeker \& Bell 1988) and the deep sea (Carreras-Colom et al. 2018; Courtene-Jones et al. 2019; Courtene-Jones et al. 2017). With almost 1000 species affected, there is no doubt that plastic pollution is pervasive and available to any kind of marine organism. Plastic pollution has been considered as one of the most urgent environmental issues by UNEP (2011), but the extent of negative effects on marine wildlife is hard to assess and sometimes might be exaggerated (Völker et al. 2019). In well-studied turtles, where all species have been found to ingest plastics, only one third (32\%) of the individuals contained plastic. Seabirds and specifi- 
cally tubenosed seabirds also belong to a well-studied species group. The individual \%FO in procellariforms is high with almost half of the birds (41.5\%) containing plastics. However, when looking into the species groups within the tubenose order there is still a large variability in the occurrence of plastics. For instance, the mollymawks and the gadfly petrels, (10 and 35 species respectively), with sample sizes $>100$, had low Ind-\%FOs of 5 $\%$ (mollymawks) and $16.4 \%$ (gadfly petrels). Other orders, such as Suliformes (gannets and cormorants) and Charadriiformes (gulls, terns, auks, etc.) have even lower Ind-\%FOs of plastics in their stomachs (respectively $20.2 \%$ and 12.3\%). Although known for regular visits at landfills and snack bars (e.g. Lenzi et al. 2016), only $16.3 \%$ of all studied gulls contained plastics at the moment of analysis. This may be explained by their feeding habits of regurgitating indigestible prey items (including plastics) on a regular basis (Barrett et al. 2007). Marine mammals and especially seal species seem to suffer more severely from entanglement (71\% of all seal species) than from ingestion $(48.4 \%$ of all seal species) of plastics. The Ind-\%FO of ingestion of plastics in marine mammals is generally low (4.4\%). Although some impressive cases of sperm whales ingesting many large plastic items (Jacobsen et al. 2010; De Stephanis et al. 2013; Unger et al. 2016), only baleen whales show a higher Ind-\%FO (16.7\%), all other species groups within the marine mammals remain well under 10\%. These low numbers, however, are not irrelevant and do not provide evidence for a lack of harm for the individual or for populations and species. Some species exhibit an ongoing high intake of plastics throughout their distribution range, exceeding the Ind-\%FO of $80 \%$, such as Laysan albatrosses (sample size $\mathrm{n}=$ 962), Tristram's storm petrels $(n=150)$, northern fulmars $(n=3095)$ and parakeet auklets (Aethia psittacula; $\mathrm{n}=325$ ). This constant uptake should be of concern as reduced fitness and plastic- associated chemicals may negatively influence the health of the population when most individuals within a population are affected. On individual level, even small amounts of plastics can be fatal (Bjorndal et al. 1994; Domènech et al. 2019; Mate 1985; Bogomolni et al. 2010; Brandão et al. 2011). Roman et al. (2019a) and Wilcox et al. (2018) predict a strongly increased chance of mortality for tubenosed birds in the southern hemisphere and turtles when ingesting plastics. An ongoing discussion is, how to deal with fibre contamination in samples. Fibres originate from e.g. clothing and due to their small weight easily become airborne. They are omnipresent in the environment (e.g. Dris et al. 2016; Bergmann et al. 2019) and available for marine organisms. Unfortunately, it is impossible to distinguish between fibres ingested by organisms and fibres as secondary contamination. Exposure time to air plays an important role (Kühn et al. 2018a; Kühn et al. 2019) and should therefore be avoided, e.g. by the use of laminar flow cabinets (Hermsen et al. 2017; Wesch et al. 2017). In recent literature it is mainly fish where fibres are presented in high numbers, often strongly dominating the plastic particle abundance in samples (Mizraji et al. 2017; Nadal et al. 2016; McGoran et al. 2017), and often without a clear description of mitigation measures to prevent airborne fibre contamination. These numbers should therefore be treated with care, as overestimation is likely. Separate recording and reporting of fibres from other particles is recommended for any future publication. 
Variation in plastic abundance in the environment is reflected by the amount of plastics found in marine organisms. These patterns are linked to input areas of plastic and currents distributing plastics (Jambeck et al. 2015; Van Sebille et al. 2012). The high concentration of plastics in Tristram's storm petrels and Laysan albatrosses are caused by high concentrations of plastics in the central north Pacific gyre (Moore et al. 2001; Eriksen et al. 2014). Linking the pollution in areas directly to plastic ingestion in species is complicated in most cases, as information is often scattered and incomplete for many regions. Species with a large distribution range are more likely investigated in several studies, as can be seen in e.g. turtles. For entanglement, Ryan (2018) calculated that most species records occurred in temperate regions and explained this by a lower number of seabird species towards the equatorial regions. Some regions appear underrepresented in plastic studies. Although plastic-accumulating gyres occur both in the North and South Pacific (van Sebille et al. 2015), 111 ingestion studies report data from the North Pacific and only 79 ingestion studies origin from the South Pacific (including unspecified Australian coasts). The same pattern occurs in the Atlantic Ocean, in the North Atlantic 193 studies report plastic ingestion and 89 ingestion studies are published from the South Atlantic (including unspecified South African coasts). Studies from the Arctic $(n=17)$ and Antarctic $(n=7)$ regions are scarce, most likely due to the inaccessibility of these regions.

As long as data are scattered and not standardized, fine-scaling of regional differences remains futile. The only species with sufficient data allowing spatial comparisons of plastic uptake, is the northern fulmar. Fulmars are used as a monitoring species to assess plastic pollution in the North Sea (Van Franeker et al. 2011; OSPAR 2017) and the same methods regarding sampling, analysis and data reporting have been applied elsewhere in the North Atlantic (Van Franeker 1985; Van Franeker et al. 2011; Acampora et al. 2016; Kühn \& Van Franeker 2012), in the North Pacific (Avery-Gomm et al. 2012; Terepocki et al. 2017; Donnelly-Greenan et al. 2014) and in the Arctic Ocean (Trevail et al. 2015a; Mallory 2008; Provencher et al. 2009; Poon et al. 2016), allowing wide-scaled comparisons between regions. When studying fulmars throughout their distribution range, spatial patterns in the abundance of plastics appear, according to the grade of pollution in certain areas. Plastic in fulmars gradually decreases towards northern latitudes (Trevail et al. 2015a). Longterm monitoring, as established in the North Sea, could be expanded to other regions were fulmars occur (Avery-Gomm et al. 2012; Avery-Gomm et al. 2018; Acampora et al. 2016). The results of this review may be helpful in identifying potential other species that are suitable as biological indicators for marine plastic pollution. To be assigned as suitable for monitoring, species should ingest plastics regularly, should be available in sufficient numbers (e.g. dead on beaches or fisheries bycatch) and should forage exclusively at sea (Van Franeker \& Meijboom 2002; Provencher et al. 2017; Matiddi et al. 2017; Bray et al. 2019).

\section{Variation in methodological setup}

Variation of methods represents the main issue that hampers analysis of data on a large scale. Some case studies are highlighted, emphasizing the different outcomes of studies, depending on their study setup. 


\section{Case study 1: Seabirds - Dissection and regurgitations}

For many seabird studies samples are collected by dissecting dead individuals. However, to study the natural diet and plastic uptake in live birds, e.g. in breeding colonies, emetics or stomach lavages are regularly applied. Another method to obtain samples is the collection of regurgitates or boluses, some seabird species produce regularly. Provencher et al. (2017) found that $70 \%(n=82)$ of the studies used dead birds from beaches, roads or colonies or being killed in hunting or bycatch. Stomach lavage or emetics were used in 9 studies and natural regurgitates were analysed in 23 studies. Beside potential harm to the individual when using invasive research methods, the full stomach content can often not be obtained in all seabird species. Procellariform seabirds have two stomachs, divided by a small constriction. As most plastic is retained in the latter stomach (the muscular gizzard), only a small fraction of plastics will be collected when regurgitations are obtained, making it difficult to compare results (Provencher et al. 2019). To emphasize the effect of method and gut morphology on Ind-\%FO, gulls, skuas and cormorants, all known to regurgitate regularly, were compared with petrels (Procellariidae) and albatrosses (Diomedeidae), both accumulating plastics. Table 10.6 shows, that the outcome in gulls, skuas and cormorants are similar, irrespective the method, as probably in both cases the complete stomach content has been available. However, in petrels and albatrosses, more birds were encountered with plastics, when dissecting the birds and analysing both stomachs, indicating that regurgitates in these species do not reveal the full plastic load.

\section{Case study 2: Seabirds - Age variation}

Sometimes it is not only the method used that can cause difference in numbers but it might for example be related to the ecology of species: young seabirds tend to have more plastics in their stomachs than adults, partly because they get fed by two plastic-ingesting parent but possibly also due to inexperience in searching for food (Kühn et al. 2015). For

Table 10.6. Comparison of two different groups of species, with different gut morphology and foraging habits. Both groups have been studied by either dissecting the birds or using regurgitates (pellets, boluses, emetics). Sample size of birds analysed are shown together with the number of individuals with plastic and their according percentage.

\begin{tabular}{|c|c|c|c|c|c|c|}
\hline \multirow[t]{2}{*}{ Taxon } & \multicolumn{3}{|c|}{ Dissection } & \multicolumn{3}{|c|}{ Regurgitates } \\
\hline & $\begin{array}{c}\text { Sample } \\
\text { size }\end{array}$ & $\begin{array}{l}\text { Individuals } \\
\text { with plastic }\end{array}$ & $\%$ & $\begin{array}{l}\text { Sample } \\
\text { size }\end{array}$ & $\begin{array}{l}\text { Individuals } \\
\text { with plastic }\end{array}$ & $\%$ \\
\hline Gulls & 1386 & 210 & 15.15 & 3404 & 572 & 16.80 \\
\hline Skuas & 110 & 9 & 8.18 & 2082 & 171 & 8.21 \\
\hline Cormorants & 84 & 10 & 11.90 & 496 & 41 & 8.27 \\
\hline Procellariidae & 14661 & 6916 & 47.17 & 1653 & 455 & 27.53 \\
\hline Albatrosses & 2878 & 1066 & 37.10 & 238 & 14 & 5.88 \\
\hline
\end{tabular}




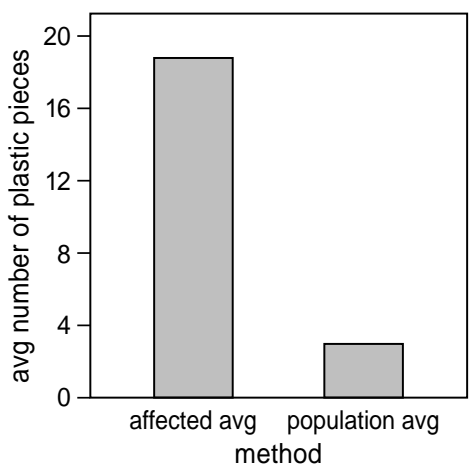

Figure 10.3. Comparison of two methods. Affected average number of pieces excludes all seabirds without plastic $(n=385)$. The population average includes all birds sampled $(n=2447)$. In total, 7244 plastic items were detected in ten studies, where the affected average has been reported but the population average could be calculated.

instance, in most studies of Laysan albatrosses, stomachs of young birds were analysed. When adding up all records of young birds from different studies (where Ind-\%FO is available), 635 birds were analysed and 96\% contained plastics (Auman et al. 1997; Lavers \& Bond 2016; Pettit et al. 1981; Rapp et al. 2017; Sileo et al. 1990). In contrast, among 222 adult Laysan albatrosses only 84\% had ingested plastics (Gould et al. 1997; Gray et al. 2012; Rapp et al. 2017). The study bias towards younger birds may explain the high Ind$\%$ FO in Laysan albatrosses. In beached northern fulmars found in the Netherlands, young birds consistently have a higher mass of ingested plastic in the stomach than adult birds (Van Franeker et al. 2011).

\section{Case study 3: Seabirds - population and affected averages}

For 10 dedicated seabird ingestion studies and 50 study records therein, the affected average of the number of ingested plastic particles was reported. However, the true population average could be derived from underlying data (Ainley et al. 1990b; Bond et al. 2010; Donnelly-Greenan et al. 2018; Floren \& Shugart 2017; Lavers \& Bond 2016; Mallory 2008; Mallory et al. 2006; Rodríguez et al. 2012; Spear et al. 1995; Verlis et al. 2013). The species $(n=43)$ belong mainly to the Procellariiformes and Charadriiformes. From these species, 2447 individuals were analysed, 385 birds contained plastics, 7244 items in total. The affected average, excluding birds without plastics is 18.82 plastic pieces. When including all birds sampled, the population average is much lower, 2.96 plastic pieces (Figure 10.3).

A significant difference $(\mathrm{P}<0.0001)$ was indicated (Wilcoxon signed rank test for nonparametric paired data https://epitools.ausvet.io/paired), with affected averages being higher than population averages, emphasizing the importance to present results including the complete study sample to avoid overestimation of numbers and second to report which 
type of average has been used to allow comparisons between studies. Reporting different or unclear metrics in plastic ingestion studies impede the structural analysis of results.

\section{Case study 4: Marine mammals - sampling method}

How much influence a specific sampling method can have on the results has been shown in one study of plastic ingestion in harbour porpoises by Van Franeker et al. (2018). Between 2003 and 2013, 654 porpoises were studied. Using a simple overflow method (to retain hard natural prey items), $6 \%$ of the porpoises contained plastics. However, after adapting the method towards a plastic-dedicated protocol using an additional sieve, that caught all overflowing material, the actual \%FO increased to $15 \%$.

\section{Case Study 5: Turtles - plastic distribution in the gastrointestinal tract}

Most studies on plastics in turtles include the complete gastrointestinal tract (GIT), others analyse the stomach content only. The complete GIT of turtles was considered in 42 studies, only the stomach in 8 studies. The remaining studies do either give no details or consider faeces, parts of the GIT or stomach flushing. Ind-\%FO was calculated for all individuals of each group. These two groups were tested for a significant difference, using 2sample $\mathrm{z}$ test to compare proportions http://epitools.ausvet.com.au/content.php?page= z-test-2 as recommended by Provencher et al. (2017).

For a total of 3639 turtles the method was specified. In 218 turtles only the stomach was analysed while 3421 turtles were analysed for plastics in the complete GIT. Out of the stomach-only turtles, $19.27 \%$ were found to contain plastics and when the whole GIT was analysed, 51.45\% contained plastics (Figure 10.4), indicating that a substantial amount of plastic may be missed out when only analysing the stomach. Therefore, the parts of GIT analysed should always be specified as for example was done by Camedda et al. (2014).

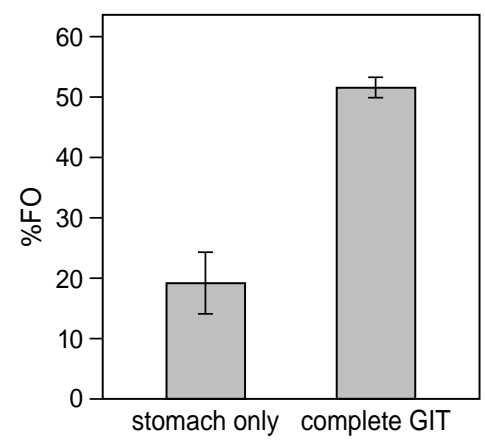

Figure 10.4. Frequency of occurrence (\%FO) of plastic ingestion of turtles (all species combined). Two methods analysing either the stomach only or the complete gastrointestinal tract (GIT) were compared. Error bars indicate the $95 \%$ confidence limits. 


\section{Conclusion}

Despite the fact that plastics are found in all regions of the ocean and are ingested by a great variety of marine organisms, the frequency and abundance of ingested plastic appears lower than sometimes suggested. Often the increasing proportion of seabird species that have at least one documented case of plastic ingestion (our Tax-\%FO), is erroneously worded as the proportion of seabird individuals having plastic in the stomach (Ind \%FO). From historic data, Wilcox et al. (2015) modelled that by $2015,90 \%$ of individuals of the worldwide seabird species would have plastic in the stomach. The model indicated that by 2050, records for ingestion would exist for $99 \%$ of seabird species. Like Wilcox et al. (2015), we do believe that with increased studies ultimately any species is likely to show individual examples of direct or indirect ingestion of plastic (see for example the increases in Tax \%FO in the few years between 2015 and 2019 in Online Supplement Table 3). But our data do not support the idea that already now nearly every individual seabird has plastics in the stomach. Many of the data considered in this review have a relatively recent origin, but nevertheless, overall, $<30 \%$ of individual seabirds, $4.4 \%$ of mammals and $32 \%$ of turtles has plastic in the stomach. Some species, like several tubenoses, seem very prone to ingest plastic debris and a large proportion of their individuals may have a substantial amount of plastic. Fortunately, these species do not represent the average current situation. This, however, gives no guarantee for the future, and should also not hide the fact, that already now some populations or species of marine wildlife may suffer from plastics in their stomachs or in their surroundings.

In this, it must be emphasized that the data on plastic ingestion discussed in this review refer to visibly detectable plastic particles in digestive tracks of marine wildlife. Although some of these plastics are certainly in the range of microplastics, this review cannot tell anything about the potential abundance of, and inflicted harm from the smallest types of plastics that may be present in the marine environment and its food chains. Large plastics, as discussed in this review, continuously degrade to smaller sized plastics and are the precursor of risks from smaller plastics. The smallest particles are potentially able to pass through tissue walls or cell membranes. Although experimental evidence indicates a serious risk of harm to organisms from such small particles, the actual impact on marine wildlife and food chains remains uncertain (e.g. GESAMP 2016; SAPEA 2019). If consistent robust methods are applied, studying patterns and trends in the frequency and abundance of visible plastic sizes ingested by marine wildlife is probably the best indicator for the risks taken with our marine environments.

\section{Acknowledgement}

S. Kühn was funded by the Dutch Science Foundation (project ALW-NWO 856.15.001) under EU Joint Program Initiative (JPI) 'Oceans' within the PLASTOX project (Direct and indirect ecotoxicological impacts of microplastics on marine organisms). Marine litter monitoring by Wageningen Marine Research is supported by the Netherlands Ministry of Infrastructure and Waterways. 


\section{Chapter 11}

\section{Synthesis}
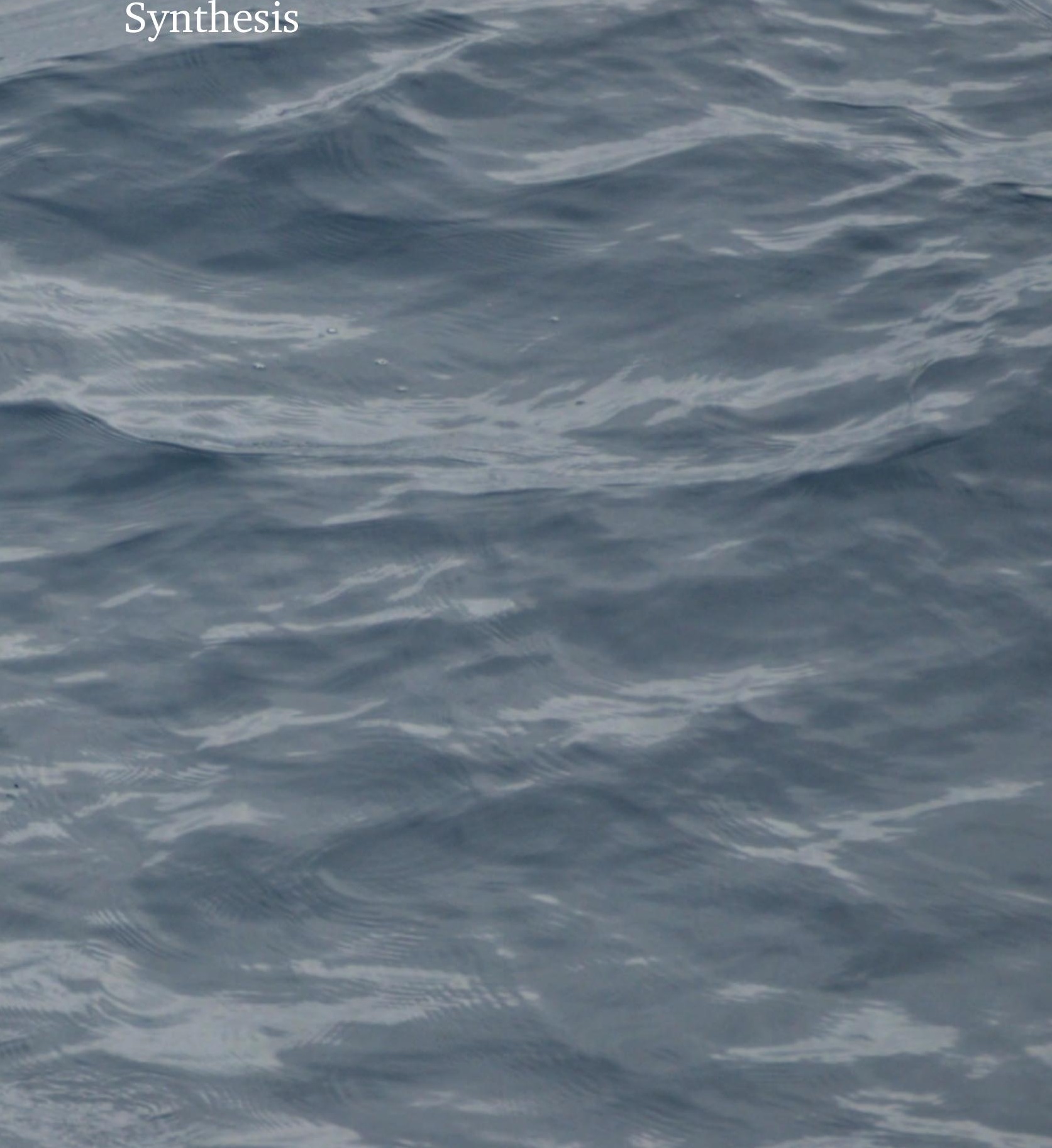
This thesis aims to investigate different pathways of plastic interaction in northern fulmars. Three main pathways are illustrated: 1 . the direct uptake of plastics by fulmars, 2 . the indirect uptake via prey, and 3. the transfer of ingested plastic-associated chemicals to fulmars. In addition, for use in exposure studies and risk assessments by all PLASTOX partners, marine-derived plastic litter was collected, milled, and analysed. As fulmars are not the only species ingesting plastics, a global review study was conducted updating the incidence and abundance levels of plastics in marine birds, mammals, and turtles.

\section{Spatial and temporal plastic patterns in marine biota}

The reason for plastic uptake in fulmars but also other species remains unclear. In turtles it has been hypothesized that turtles confuse plastic bags with jelly fish (Schuyler et al. 2012; Schuyler et al. 2014). The foraging technique (e.g. surface seizing or plunge diving) may also contribute to different means of plastic uptake (Day et al. 1985) and specialized feeders are thought to be less prone to plastic ingestion than species with a more general diet (Ryan 1987; Kühn et al. 2015). The uptake of plastic by fulmars is driven by the availability of plastic in the surrounding environment. Fulmars from polluted areas carry more plastic in their stomachs than fulmars from remote areas, far from human urbanization. This has been demonstrated in several studies from the North Atlantic and North Pacific. The overall frequency of occurrence ( $\% \mathrm{FO}$ ) of plastics in fulmars on a global scale is $82 \%$ and on average these birds carry 20.2 plastic items per stomach (Chapter 10). Many studies from the southern distribution range of fulmars, e.g. the North Sea and the coast of North Carolina in the North Atlantic and the Californian coast of the North Pacific report high \%FOs ranging from 84 to 95\% (Van Franeker et al. 2011; Acampora et al. 2016; Moser \& Lee 1992; Donnelly-Greenan et al. 2014; Terepocki et al. 2017; Avery-Gomm et al. 2012; Bond et al. 2014). In contrast, most studies from (sub)Arctic regions in the North Atlantic and North Pacific report lower \%FOs, ranging from 31 to 79\% (Mallory et al. 2006; Mallory 2008; Chapter 2; Day 1980), although there are some high plastic records from high Arctic regions (Provencher et al. 2009; Poon et al. 2016; Chapter 3). Unfortunately, remoteness does not always prevent the high uptake of plastics. Ocean currents, transporting plastic litter from the polluted south, may contribute to these high numbers (Obbard 2018; Trevail et al. 2015a; Trevail et al. 2015bTrevail et al. 2015a; Trevail et al. 2015b; Chapter 3). Also seasonal changes may play a role, as fulmars wintering in the south carry high loads of plastic (and associated pollutants; Blais et al. 2005) to the pristine northern regions where they breed and forage throughout summer. After one month, most particles are likely to be excreted (Van Franeker et al. 2011; Van Franeker \& Law 2015) or may be fed to offspring. Regional and temporal patterns have also been detected in the polymer types ingested by northern fulmars and their southern relatives (Chapter 7). Polyethylene (PE) and polypropylene (PP) were the most dominant polymer types encountered in all regions and time series. The differences in polymer proportions were driven by plastic categories. Pellets (Figure 11.1), increased the propor- 


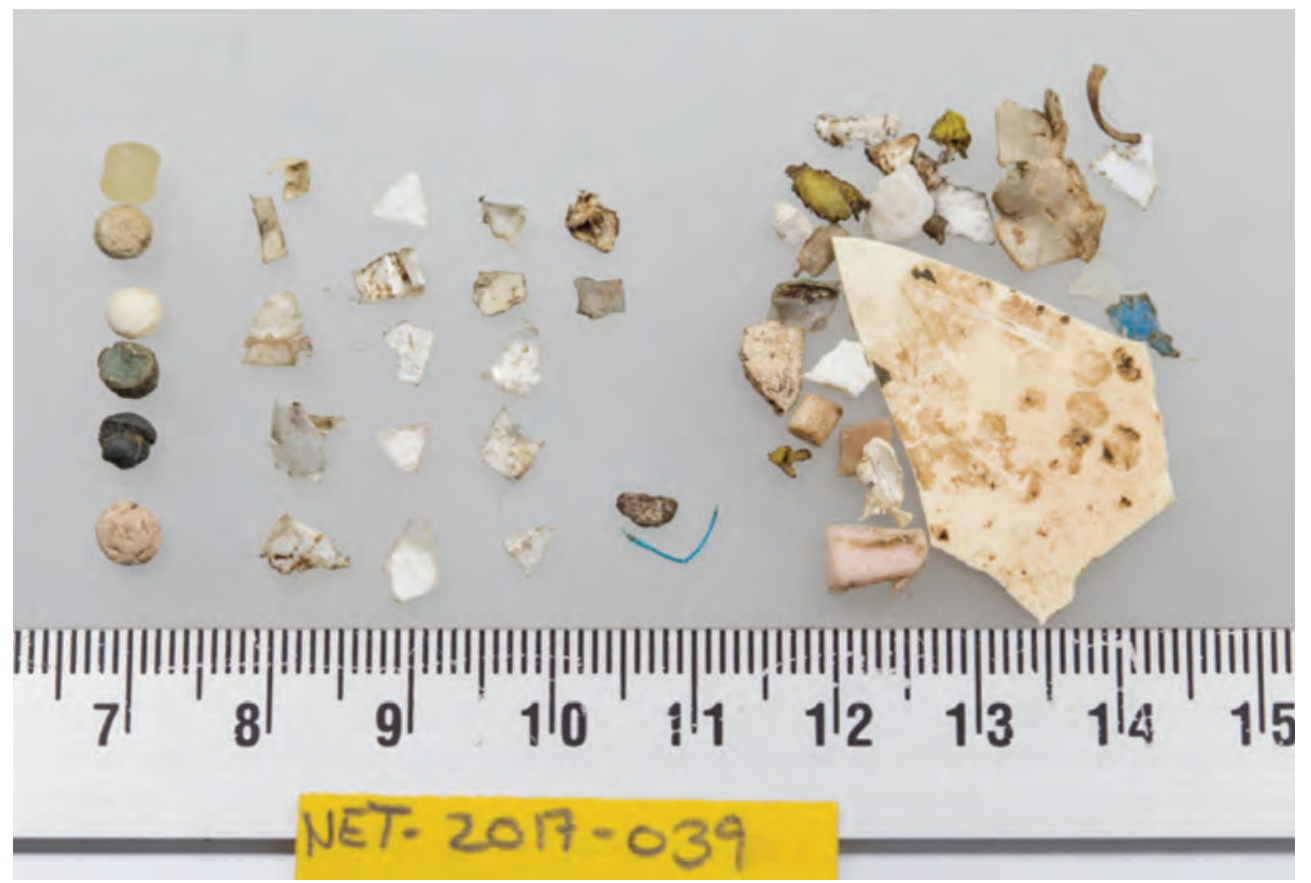

Figure 11.1. Stomach content of an adult fulmar, collected in 2017 on the Dutch coast. On the left, pellets are shown. In the centre, pieces of plastic sheets are depicted with, on the centre bottom one thread and one piece of foam. To the right, several hard plastic fragments are shown. Total mass of the plastic is 0.6286 gram, about two times the average mass of plastics found in North Sea fulmars.

tion of PE and PP in the sample as shown for northern fulmars from the Netherlands the 1980s and for seabird species in the Southern Ocean. Within a few decades, changes in the composition of seabird stomach contents, towards less pellets were observed (Ryan 2008; Van Franeker \& Law 2015). These results highlight, that political and economic measures to prevent the loss of valuable plastic stocks can result in actual improvements of the environment.

Sheets and especially threads (commonly ingested by northern fulmars from the Netherlands in the 1990s and later in Iceland and Svalbard) were usually made of PP and to a lesser extent of PE. Polyvinylchloride (PVC) was found to be the third most common plastic type in fulmars.

In fish, the spatial pattern is even more complex. When comparing fish from the Arctic Ocean (Chapter 4) with fish from the North Sea (Chapter 6), the difference in plastic uptake is less obvious than in seabirds. In the North Sea, $1.8 \%$ of the fish we studied were found with plastics, while $2.8 \%$ of polar cod (Boreogadus saida) from Svalbard and from underneath the Arctic sea ice contained plastics. Sea ice has been suspected to concentrate high numbers of plastic and release those particles with increasing sea-ice melt (Cózar et al. 2017; Obbard et al. 2014). One dedicated plastic study conducted in eastern Greenland 
found plastic in $18 \%$ of polar cod stomachs, however, the majority (88\%) of these plastics were fibres (Morgana et al. 2018). Excluding fibres, as done for our polar cod samples, the results are comparable to ours (on average 0.01 and 0.03 plastics per fish for Morgana et al. (2018) and our results respectively).

\section{The need for standardisation in plastic research}

As shown in Chapter 10, standardisation of methods in plastic ingestion studies is needed and often a key recommendation from researchers, however, has not yet been achieved. Standardisation requires comparable approaches in all steps of the study design, from plastic extraction from stomachs and the analysis of the plastics encountered, to data analysis and reporting (Provencher et al. 2017).

Dissection techniques for seabirds, and especially Procellariiformes, usually refer to the manual by Van Franeker (2004). As the World Seabird Union Specialist Committee on Seabirds and Plastic Pollution, we published two papers on the stomach content analysis and data reporting (Provencher et al. 2017; Provencher et al. 2019). The benefits of standardisation are demonstrated in fulmar studies (Van Franeker et al. 2011). Similar standardisation approaches allow for the study of spatial and temporal patterns and are thereby extremely valuable for policy-makers, as they can inform on baseline data and detect changes (Van Franeker \& Kühn 2019).

For fish, currently no standardised protocol is available. However, policy-makers are considering fish as a potential monitoring tool for plastics in the water column and on the seafloor as part of the European Marine Strategy Framework Directive (MSFD; Galgani et al. 2013a). Methodological differences hamper the comparison in many studies. The results of Chapter 6 , show a low abundance of plastics in North Sea fish species $(1.8 \%$ for all species and a maximum of $12.3 \%$ in cod (Gadus morhua). For this study, we combined two earlier studies with a new dataset, showing the additional value in terms of comparing and combining datasets. Creating long-term datasets, allow long-term temporal and large-scale spatial analyses. The comparison of our data with other studies remains complicated, due to methodological differences. Based on our results, we decided to treat fibres separately to avoid overestimation of plastics caused by airborne fibre contamination. Chapters $4 \& 6$ demonstrated that the time span needed to process a sample influenced the total number of fibres, not the number of individuals included in the sample. It has been shown that laminar air flow cabinets might reduce the number of fibre contamination almost to zero and studies using these cabinets reported generally lower numbers of fibres (Avio et al. 2015; Cannon et al. 2016; Hermsen et al. 2017; Wesch et al. 2017). Although Wieczorek et al. (2018) detected many fibres while working under laminar air flow, they point to the high abundance of plastics with a smaller cut-off size for plastics and the increased local occurrence of plastic in their study area. With advances in modern technology, researchers are now able to detect plastics down to a few micrometres (Löder \& Gerdts 2015). However, the interpretation of increased occurrences of plastics, 
especially those reported in remote environments (e.g. Bergmann et al. 2017c), should be done with care as these numbers do not necessarily reflect growing numbers of plastics but rather the development of these new techniques. Method standardisation is a slow process. For example, researchers proposed the use of potassium hydroxide $(\mathrm{KOH})$ as an appropriate method in 2013 (Foekema et al. 2013) and although some incorporated the proposed methods (Besseling et al. 2015; Rochman et al. 2015; Tahir \& Rochman 2015), thorough testing of the method only started in 2016. Dehaut et al. (2016) and Karami et al. (2017) studied the properties of plastics before and after exposure to $\mathrm{KOH}$ and other solutions, but only for a restricted number of plastic types. At the same time, we tested more than 60 plastic types and shapes and some natural prey items, to see how good these plastics can withstand $\mathrm{KOH}$ (Chapter 5). Additionally, we tested items that first had been exposed to stomach oil of fulmars and in a second step to $\mathrm{KOH}$ to make sure that oil does not affect the treatment. Results of the three studies show that $\mathrm{KOH}$ is the most suitable dissolvent for organic material and that most plastics (except some bioplastics) can withstand the $\mathrm{KOH}$ treatment. Since then, $\mathrm{KOH}$ has often been used for the treatment of organic samples, mainly to dissolve fish tissue (Bour et al. 2018; van der Hal et al. 2018 Collicutt et al. 2019; Giani et al. 2019; Chapter 6). The disadvantage of $\mathrm{KOH}$ is the loss of biological information for e.g. diet studies. Therefore, $\mathrm{KOH}$ was not used in Chapter 4, as the natural diet of polar cod was studied simultaneously.

The above-mentioned examples show that method development is necessary to reliably quantify and report data on plastic ingestion. Plastic research, specifically microplastic research, is relatively new, but the body of literature is growing at accelerating rate, reflecting the high interest from scientists, policy-makers, and the general public. This has led to an uncontrolled growth of various, often under-tested methodologies. Future aims for scientists should be to identify suitable methods to detect plastics in the environment and to standardize methods, allowing for large-scale comparisons and the evaluation of potential impacts of plastics on organisms (Chapter 10).

\section{Trophic transfer of plastics, from fish to seabirds}

In this thesis, the focus was on two species groups: seabirds and fish. Those two are inevitably linked to each other, by a predator-prey relationship whereby the seabirds, including the fulmar, are highly dependent on fish as an energy-rich food source (Cury et al. 2011). However, in rare occasions, the tables are turned, when large predatory fish may predate on seabirds (Dale et al. 2011; Duffy \& Taylor 2015) and sometimes, plastics ingested by birds are then found in predatory fish (Perry et al. 2013). This thesis focuses on the potential contribution of plastic-ingesting fish on the total load of plastics in fulmars. Fulmars feed on a variety of fish species, including polar cod (Figure 11.2; Chapter 4) and many North Sea fish species studied in Chapter 6.

The number of plastics in fulmars is high. In the North Sea, 93\% of fulmars contain plastic, on average 33 particles per stomach (OSPAR 2017). In contrary, only 1.8\% of 


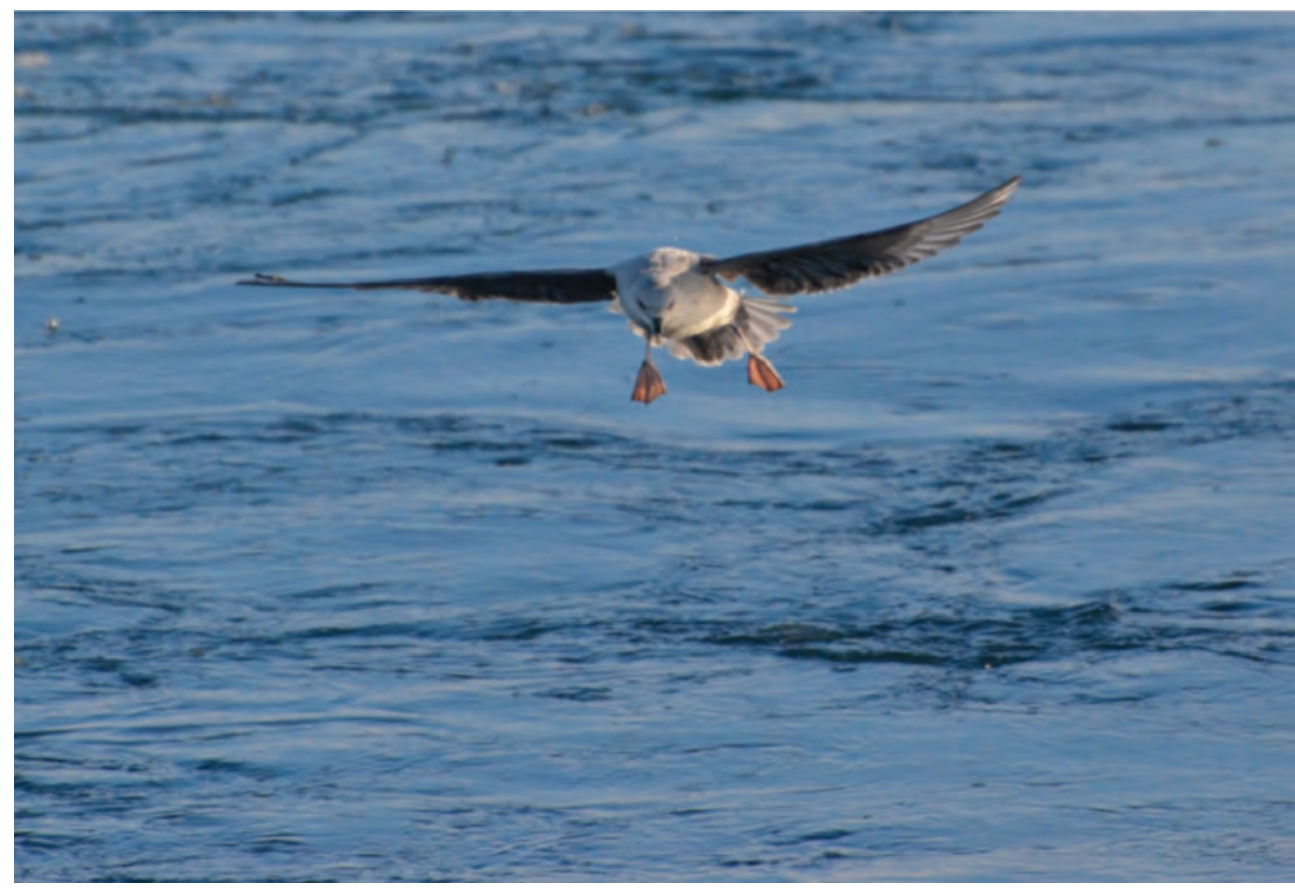

Figure 11.2. A northern fulmar searching for polar cod, at the stern of the icebreaking research vessel Polarstern. Breaking the ice revealed many polar cods, apparently confused by the sudden disturbance: An easy meal for many kittiwakes and fulmars, following the ship.

North Sea fish were found with ingested plastics, on average 0.022 plastic particles per stomach (Chapter 6). A North Sea fulmar therefore would have to consume 2525 fishes, before possibly encountering a piece of plastic.

Plastic items encountered in fish stomachs in Chapter 4 \& 6 were tiny. The smallest particle found was $0.125 \mathrm{~mm}$ and the average size of $3.18 \mathrm{~mm}$ was strongly influenced by two long threads encountered in two individuals (Chapter 6). In Chapter 6, the maximum plastic length was available for 79 pieces, of which $41.8 \%$ were smaller than a millimetre and a total of $81.1 \%$ did not exceed the $3 \mathrm{~mm}$. Plastics in fulmars have an average maximum length of 3 to $5 \mathrm{~mm}$ (Bravo Rebolledo 2011; Terepocki et al. 2017), smaller particles are assumed to be not retained in the stomach and to be excreted quickly (Van Franeker \& Law 2015). From my two studies, it seems unlikely that a substantial part of the plastics encountered in fulmars originated from their prey, due to the low number and small size of the plastic and if so, was likely excreted quickly. Trophic transfer in the visible size range of plastic therefore does not appear to be a major pathway of plastics in fulmars.

While this project did not directly test any harm to the seabird from plastic-associated chemicals which may have been be transferred from fish, we do recognize the potential for transfer as seen in work on fish and crustaceans (Batel et al. 2016). 


\section{Potential harm from plastic ingestion to fulmars}

The Good Environmental Status in the marine environments of the EU has been defined as (MSFD GES Technical Subgroup on Marine Litter 2011):

'Properties and quantities of marine litter do not cause harm to the coastal and marine environment'.

But in the same report it is stated:

'There is no final consolidated common understanding about what is harm in regard to marine litter [...]'.

These two quotes perfectly illustrate the difficulty in science and policy to identify and quantify potential harm associated with plastics in the environment and in marine biota. Browne et al. (2015) state that impacts occur when exposure of organisms to debris causes a disturbance of sufficient magnitude to overcome inertia in that level of biological organization, and therefore elicits a response'. In some cases, the impact of plastic is evident, as it may be the case for entanglement of the marine biota in plastic. Entangled individuals usually suffer from impaired movement when searching for food or hiding from predators, potentially leading to death (Kühn et al. 2015; Ryan 2018; Chapter 10). Although more visible than ingestion, it is mostly only a small number of individuals within a population that suffers from entanglement (Ryan 2018).

Harm caused by ingested plastics is much more difficult to understand. The potential effects include direct mortality and sub-lethal effects such as decreased stomach volume and digestion efficiency, false feeling of satiation or internal injuries (Kühn et al. 2015). Additionally, the leakage of potentially hazardous chemicals from plastics to animal tissue is possible. All these effects can lead to decreased body condition in marine biota and consequently to death, when e.g. external circumstances deteriorate. When many individuals of a population are affected by these sub-lethal effects, population effects are more likely (Browne et al. 2015; Chapter 10). Many chemicals have been shown to affect the health of an organism. For example, they are known to be endocrine disruptive, carcinogenic, mutagenic, and can ultimately lead to increased mortality (Hermabessiere et al. 2017). In birds, experimental exposure to plastics revealed decreased appetite and growth rate (Ryan 1988) and the increased occurrence of male reproductive cysts and reduced chick growth (Roman et al. 2019b).

For this thesis, I evaluated the type of plastic (Chapter 7) ingested by fulmars and the pathway of chemicals towards the fulmar body (Chapter 9). In many effect studies, laboratory experiments are executed with unrealistically high concentrations of clean, homogenous, and pristine plastics. Reasons for this approach are the desirable control of circumstances, the elimination of factors disturbing the experiment, and the ready-availability of plastic material from several commercial producers. However, there are some major 
concerns with this concept, primarily that these plastic items in no way reflect realistic circumstances (Phuong et al. 2016; Jahnke et al. 2017). In reality plastics ingested by marine organisms can be of every shape, size and polymer composition and will contain variable quantities of added and adsorbed chemicals attributing to their properties, such as colour, flexibility, flammability and degradation (Rani et al. 2015; Chapter 8). In some model studies no transfer of classical plastic-associated substances bisphenol A and nonylphenol has been found for fish and polychaetes (Koelmans et al. 2014) and transfer might even be reversed according to these studies, actually 'cleaning' organisms from persistent organic pollutants (POPs; Koelmans et al. 2013; Mohamed Nor \& Koelmans 2019). However, these modelling studies are based on experimental setups with pristine plastics. The applicability of these studies for wild marine organisms, ingesting degraded, post-consumer plastics, remains unclear. Tanaka et al. (2019) analysed plastic pieces ingested by procellariform seabirds, including fulmars from the Faroe Islands, and found several plastic additives such as UV stabilizers, styrene oligomers, and flame retardants in the plastics. Herzke et al. (2016) studied the uptake of chemicals in fulmars and found that ingested amounts of plastics were not correlated with the occurrence of POPs in liver and muscle tissue. However, in a comparable study by Tanaka et al. (2013), some congeners of polybrominated flame retardants were found in the adipose fat tissue of short-tailed shearwaters (Ardenna tenuirostris), that could be linked to the plastics found in the stomachs of these birds. With this research approach a snapshot (plastic in stomach) is compared to long-term accumulation (POPs in tissues), which may explain the difficulty in finding relationships between substances in plastics and in tissue. Exposure studies were executed by Tanaka et al. (2015) but with restricted focus on pristine plastics which were artificially contaminated with brominated flame retardants. In Chapter 9, we investigated the transfer of different plastic additives to the stomach oil of fulmars. We used the environmentally relevant microplastic mixture we created (Chapter 8). Similar to Tanaka et al. (2015), our exposure studies shows, that stomach oil of procellariform seabirds facilitates the transfer of chemicals from plastics to birds. Additives demonstrated to leach from plastic to stomach oil include plasticizers, antioxidants, UV stabilizers, flame retardants, and preservatives. For example PVC, the third most common polymer type ingested by fulmars, comprises $8.6 \%$ of all plastic items ingested, or $6.6 \%$ of the mass ingested (Chapter 7). PVC contains high quantities of phthalate plasticizers (Hermabessiere et al. 2017), that have been shown to leach into the stomach oil (Chapter 8). These results should be of concern, as already small quantities of phthalates have been shown to be endocrine disruptive (Oehlmann et al. 2009). Importantly, our leaching experiments used stomach oils from fulmars that already contained contaminants derived from the food web plus those from plastics in the stomachs. Nevertheless additional pollutants leached from the experimental plastic into this oil.

In chapter 9, it is demonstrated, that chemicals can be transferred from ingested plastics into fulmars. Procellariiformes are the order of seabirds with the highest occurrence of plastic (Chapter 10), and the high uptake of plastic together with the production of stomach oils may contribute to potential sub-lethal effects in this seabird family. Harm to 
wildlife (ultimately including humans) is a controversial issue that is frequently discussed in science, politics and media. However consequences of these discussions cannot change the basic fact that plastic in the oceans is a purely man-made problem and therefore any level of harm is unnecessary and avoidable. Complications in providing hard scientific evidence of harm should be no reason to lose focus in reducing plastic pollution.

\section{Monitoring and Policy}

In the previous chapters, I have highlighted and summarized pathways of plastic uptake in seabirds and their prey. Many studies on plastic ingestion by marine biota have caused concern to the general public and policy-makers around the world. Plastic pollution is perceived as one of the most important environmental problems (UNEP 2011). The general public is worried about plastic pollution and potential consequences, including for their own health. This results in a discrepancy between those concerns and the scientific output which often is unable to provide clear evidence of harm (Völker et al. 2019).

The results of this thesis can contribute in different ways to plastic pollution policies. The data from Chapter $2 \& 3$ can be used as a baseline for future plastic monitoring schemes in Svalbard and Iceland. Results from Chapter 10 provide information on which species most frequently ingest plastics and therefore might be considered for monitoring. Trends in marine litter are difficult to obtain, often due to the discrepancy in methods (Chapter 10). However, the agreement of European countries to monitor plastic in fulmars in the framework of OSPAR and the MSFD, has led to a long-term dataset that has created the opportunity to study spatial and temporal trends in plastic ingestion, including changes in plastic type proportions throughout the years (Van Franeker et al. 2011; Van Franeker \& Law 2015; Chapter 7). Plastic in fulmars increased in the 1990s, but decreased towards a lower and consistent level in the early 2000s. With decreasing numbers of pellets, an overall decreasing trend of plastic in fulmars has been observed, reflecting the great effort by policy-makers and private initiatives to reduce plastic input to the North Sea (Van Franeker \& Kühn 2019). This EcoQO could be reached in a few decades on the condition that all efforts to decrease marine plastic pollution continue with the same urgency (Figure 11.3; Van Franeker \& Kühn 2019).

To monitor regions or habitats where fulmars do not occur or forage, e.g. the Baltic \& Mediterranean Sea, or to monitor plastic occurrence on the seafloor, we propose that fish species, such as the demersal Atlantic cod (Gadus morhua) and pelagic sprat (Sprattus sprattus) could complement the monitoring scheme. Methodology and data treatment (e.g. in regards to fibre contamination) should be agreed on between EU member states (Chapter 6). 


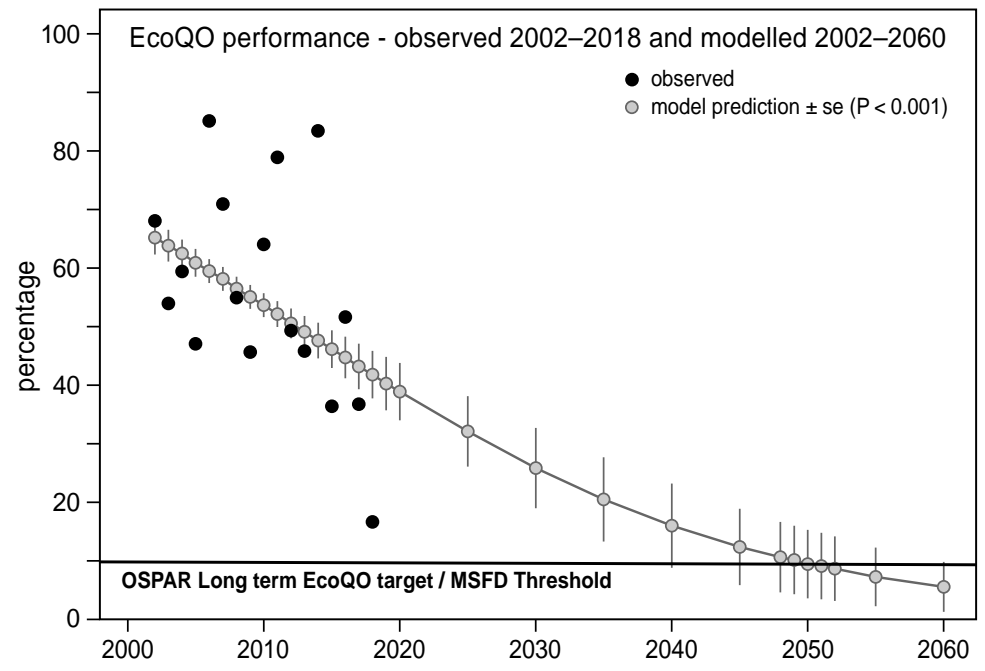

Figure 11.3. GLM model analyses of annual EcoQO performance of fulmars using 17 years of EcoQO data (2002-2018) from the Netherlands. Observed data are the black circles in the graph. The predicted annual values and standard errors are shown by grey circles connected by a trendline. Source: Van Franeker \& Kühn 2019.

\section{Future research \& recommendations}

This thesis is a product of many years of research. During this time, some answers were given, but also questions were raised. For example 'why can't or why don't fulmars distinguish between natural food and plastic?' or 'does plastic ingestion contribute to the decline of (seabird) species, and if so, to what extent?'. More specific questions concern the impact of plastics on the environment. From studies conducted in this thesis, it is clear that plastics are pervasive and occur in all habitats (Chapters 4, $6 \& 10$ ), even in remote locations (Chapters 2, 3, 4, 7 \& 10). Harm in marine wildlife is hard to identify, as many natural and anthropogenic factors can intertwine. Disentanglement of these factors is complex and requires major efforts, both from scientists and governments. We showed that ingested plastics are a pathway for chemicals to enter the fulmars' digestive system, followed by questions regarding the effects of these substances on the organism. Trophic transfer of plastics seems to be a minor issue between fulmars and their fish prey, however, trophic transfer of plastic-associated chemicals should be assessed. Therefore plastic-specific substances must be identified, as many POP's are already available in seawater and prey fish, not necessarily linking plastics and chemical uptake. Future risk assessments should rely on realistic and relevant characteristics and concentrations of plastics or plastic-associated substances (Chapter 8). Closing the gap between laboratory research and the application of gained knowledge to wildlife should be a priority. 
Long-term monitoring has been shown to be extremely valuable for scientists and policy makers. The value of long-term monitoring should be acknowledged by governments and funding agencies and should be supported accordingly. Consistency in data collection and analysis over a long period of time and a large area, allow a profound view on questions of changes in seasonality, temporal and spatial trends, and biological factors of the organism studied, such as age, sex, size, foraging behaviour etc. If fish are indeed considered as a monitoring organism, consistent sampling in terms of space and time, as well as standardized data analyses are a prerequisite. Therefore, more data on spatial and temporal variability is necessary to agree on a sound monitoring system (Chapter 6).

\section{Concluding remarks}

The research presented in this thesis has shown that fulmars belong to the most affected marine species with regard to the ingestion of marine plastic litter. Even in remote locations, the majority of fulmars ingest plastics regularly, reflecting the global scale of plastic debris in the ocean. The transfer of substances associated to plastics has been observed and may pose an additional threat to fulmar populations.

Although plastic uptake in fish may not represent a major pathway of plastic towards fulmars, fish may be valuable as monitoring organism, extending potential monitoring efforts outside of the fulmars' distribution range. To study plastic ingestion in marine organisms, standardized approaches are necessary to produce reliable results that are comparable to studies from other locations or from the past. By testing new methods for their suitability and the creation of a litter-derived microplastic mixture, available to PLASTOX partners and applied in our own studies, comparability of studies has been enhanced.

In the Dutch long term fulmar monitoring program, a slow but significant decrease of plastics has been observed (Van Franeker \& Kühn 2019). This may be considered a reward for all efforts by governments and society to reduce plastics and as such should motivate the continuation of these efforts. The fulmar, perfectly adapted to a life at sea, but not readily prepared to humans' waste society, can help us to disseminate this message and contribute to increase our understanding of the impacts that plastics can have on wildlife. 

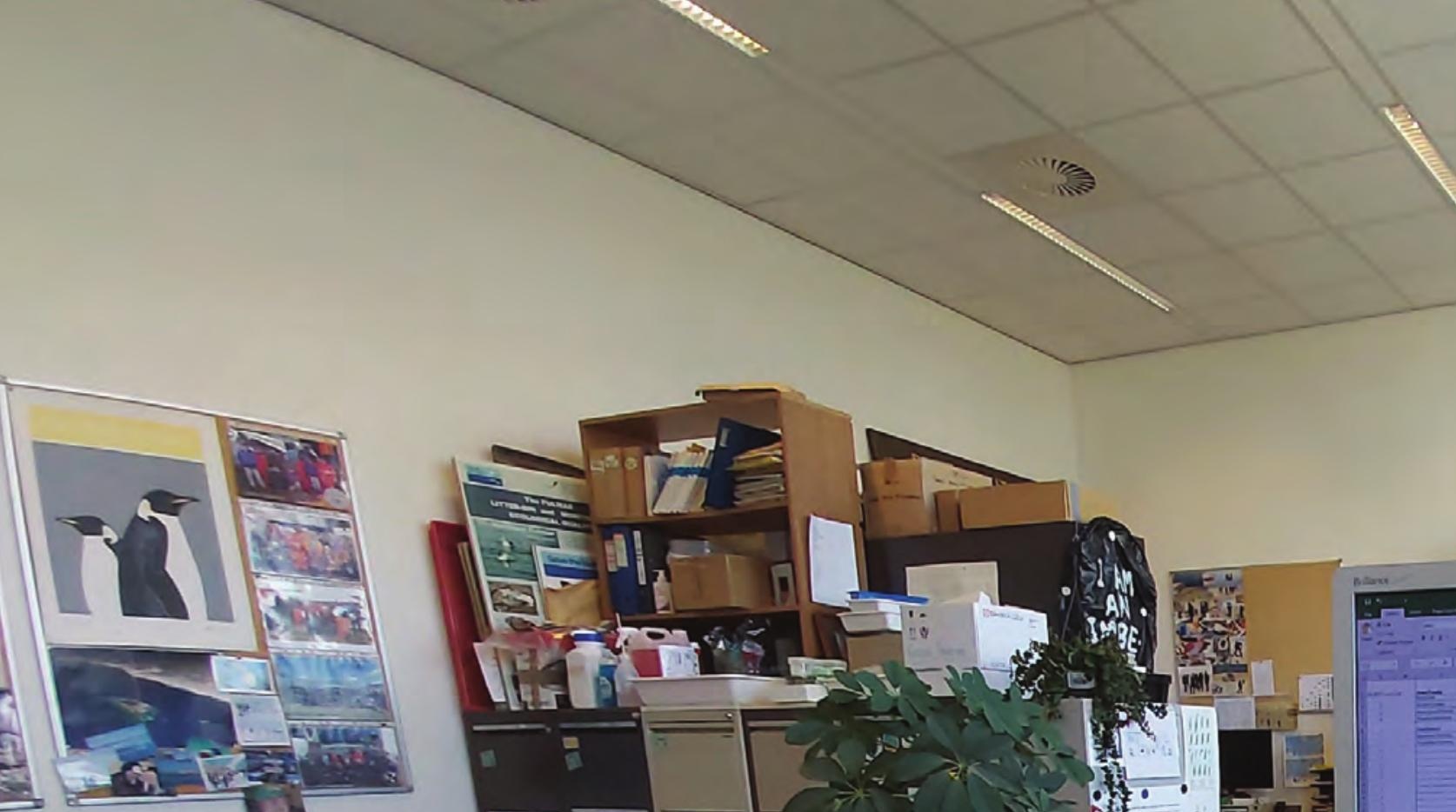

.

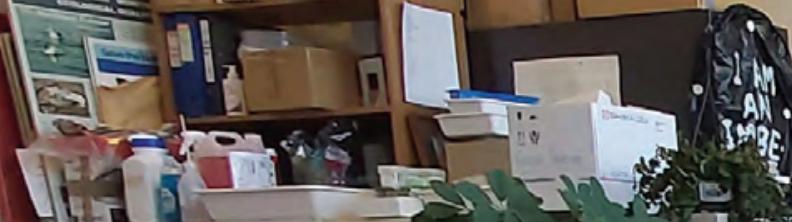

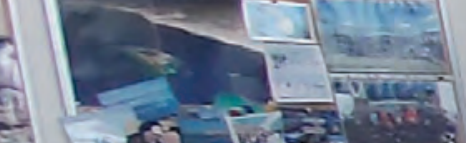

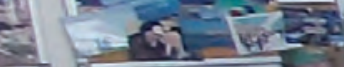

u
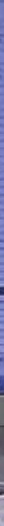

- 1

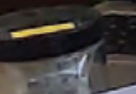

들

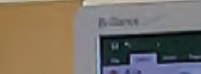

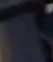

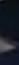

e
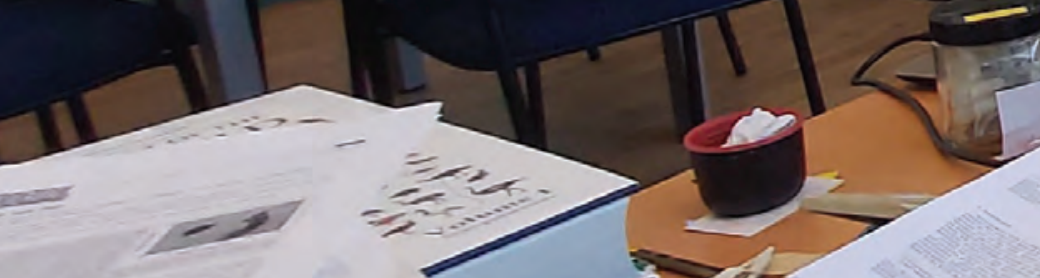


\section{A}

Abreo NAS, Macusi ED, Blatchley DB, Cuenca GC (2016) First Evidence of Plastic Ingestion by the Rare Deraniyagala's Beaked Whale (Mesoplodon hotaula). IAMURE International Journal of Ecology and Conservation 19: 16-36

Abreo NAS, Blatchley D, Superio MD (2019) Stranded whale shark (Rhincodon typus) reveals vulnerability of filter-feeding elasmobranchs to marine litter in the Philippines. Marine Pollution Bulletin 141: 79-83

Abts G (2010) Kunststoff-Wissen für Einsteiger. Carl Hanser Verlag, München

Acampora H, Lyashevska O, Van Franeker JA, O'Connor I (2016) The use of beached bird surveys for marine plastic litter monitoring in Ireland. Marine Environmental Research 120: 122-129

Accinelli C, Sacca ML, Mencarelli M, Vicari A (2012) Deterioration of bioplastic carrier bags in the environment and assessment of a new recycling alternative. Chemosphere 89: 136-143

Adams WJ, Biddinger GR, Robillard KA, Gorsuch JW (1995) A summary of the acute toxicity of 14 phthalate esters to representative aquatic organisms. Environmental Toxicology and Chemistry 14: 1569-1574

Ainley DG, Fraser WR, Spear LB (1990a) The incidence of plastic in the diets of Antarctic seabirds. Shomura RS, Godfrey ML, Proceedings of the Workshop on the fate and impact of marine debris, Honolulu, Hawaii, pp 682-691

Ainley DG, Spear LB, Ribic CA (1990b) The incidence of plastic in the diets of pelagic seabirds in the eastern equatorial Pacific region. Shomura RS, Godfrey ML, Proceedings of the Workshop on the fate and impact of marine debris, Honolulu, Hawaii, pp 653-665

Alomar C, Deudero S (2017) Evidence of microplastic ingestion in the shark Galeus melastomus Rafinesque, 1810 in the continental shelf off the western Mediterranean Sea. Environmental Pollution 223: 223-229

Alomar C, Sureda A, Capó X, Guijarro B, Tejada S, Deudero S (2017) Microplastic ingestion by Mullus surmuletus Linnaeus, 1758 fish and its potential for causing oxidative stress. Environmental Research 159: 135-142

Amélineau F, Bonnet D, Heitz O, Mortreux V, Harding AM, Karnovsky N, Walkusz W, Fort J, Gremillet D (2016) Microplastic pollution in the Greenland Sea: Background levels and selective contamination of planktivorous diving seabirds. Environmental Pollution 219: 1131-1139

Anastasopoulou A, Mytilineou C, Smith CJ, Papadopoulou KN (2013) Plastic debris ingested by deepwater fish of the Ionian Sea (Eastern Mediterranean). Deep Sea Research Part I: Oceanographic Research Papers 74: 11-13

Andrady AL, Neal MA (2009) Applications and societal benefits of plastics. Philos Trans R Soc Lond B Biol Sci 364: 1977-1984

Andresen JA, Grundmann A, Bester K (2004) Organophosphorus flame retardants and plasticisers in surface waters. Science of The Total Environment 332: 155-166

Artham T, Doble M (2009) Fouling and Degradation of Polycarbonate in Seawater: Field and Lab Studies. Journal of Polymers and the Environment 17: 170-180

Arthur C, Baker J, Bamford H (2009) Proceedings of the International Research Workshop on the Occurrence, Effects, and Fate of Microplastic Marine Debris, September 9-11, 2008. Arthur C, Baker J, Bamford H, University of Washington Tacoma, Tacoma, WA, USA, pp 49

Auman HJ, Ludwig JP, Giesy JP, Colborn T (1997) Plastic ingestion by Laysan Albatross chicks on Sand Island, Midway Atoll, in 1994 and 1995. In: Robinson G, Gales R (eds) Albatross Biology and Conservation. Surrey Beatty and Sons, Chipping Norton

Avery-Gomm S, O'Hara PD, Kleine L, Bowes V, Wilson LK, Barry KL (2012) Northern fulmars as biological monitors of trends of plastic pollution in the eastern North Pacific. Marine Pollution Bulletin 64: 1776-1781

Avery-Gomm S, Valliant M, Schacter CR, Robbins KF, Liboiron M, Daoust P-Y, Rios LM, Jones IL (2016) A study of wrecked Dovekies (Alle alle) in the western North Atlantic highlights the importance of using standardized methods to quantify plastic ingestion. Marine Pollution Bulletin 113: 75-80 
Avery-Gomm S, Provencher JF, Liboiron M, Poon FE, Smith PA (2018) Plastic pollution in the Labrador Sea: An assessment using the seabird northern fulmar Fulmarus glacialis as a biological monitoring species. Marine Pollution Bulletin 127: 817-822

Avio CG, Gorbi S, Regoli F (2015) Experimental development of a new protocol for extraction and characterization of microplastics in fish tissues: first observations in commercial species from Adriatic Sea. Marine Environmental Research 111: 18-26

Azevedo-Santos VM, Gonçalves GRL, Manoel PS, Andrade MC, Lima FP, Pelicice FM (2019) Plastic ingestion by fish: A global assessment. Environmental Pollution: 112994

\section{B}

Baalkhuyur FM, Dohaish E-JAB, Elhalwagy ME, Alikunhi NM, AlSuwailem AM, Røstad A, Coker DJ, Berumen ML, Duarte CM (2018) Microplastic in the gastrointestinal tract of fishes along the Saudi Arabian Red Sea coast. Marine Pollution Bulletin 131: 407-415

Bakir A, Rowland SJ, Thompson RC (2014) Enhanced desorption of persistent organic pollutants from microplastics under simulated physiological conditions. Environmental Pollution 185: 16-23

Bakir A, O'Connor IA, Rowland SJ, Hendriks AJ, Thompson RC (2016) Relative importance of microplastics as a pathway for the transfer of hydrophobic organic chemicals to marine life. Environmental Pollution 219: 56-65

Balazs GH (1985) Impact of ocean debris on marine turtles: Entanglement and ingestion. Shomura RS, Yoshida HO, Proceedings of the Workshop on the fate and impact of Marine debris, 26-29 November 1984, Honolulu, Hawaii NOAA Technical Memo NOAA-TM-NMFS-SWFC-54 pp 387-429

Baltz DM, Morejohn GV (1976) Evidence from seabirds and plastic particle pollution off Central California. Western Birds 7: 11-112

Barnes DK (2002) Biodiversity: Invasions by marine life on plastic debris. Nature 416: 808-809

Barnes DK, Galgani F, Thompson RC, Barlaz M (2009) Accumulation and fragmentation of plastic debris in global environments. Philos Trans R Soc Lond B Biol Sci 364: 1985-1998

Barnes DKA (2005) Remote Islands reveal rapid rise of Southern Hemisphere, sea debris. The Scientific World Journal 5: 915-921

Barrett RT, Camphuysen K, Anker-Nilssen T, Chardine JW, Furness RW, Garthe S, Hüppop O, Leopold MF, Montevecchi WA, Veit RR (2007) Diet studies of seabirds: a review and recommendations. ICES Journal of Marine Science 64: 1675-1691

Batel A, Linti F, Scherer M, Erdinger L, Braunbeck T (2016) Transfer of benzo [a] pyrene from microplastics to Artemia nauplii and further to zebrafish via a trophic food web experiment: CYP1A induction and visual tracking of persistent organic pollutants. Environmental Toxicology and Chemistry 35: 1656-1666

Battisti C, Staffieri E, Poeta G, Sorace A, Luiselli L, Amori G (2019) Interactions between anthropogenic litter and birds: a global review with a 'black-list' of species. Marine Pollution Bulletin 138: 93-114

Baxter A (2009) Report on the blue whale stranding northwest coast of the South Island, New Zealand, May 2009. Department of Conservation, Nelson, New Zealand, pp 4

Bergmann M, Klages M (2012) Increase of litter at the Arctic deep-sea observatory HAUSGARTEN. Marine Pollution Bulletin 64: 2734-2741

Bergmann M, Lutz B, Tekman MB, Gutow L (2017a) Citizen scientists reveal: Marine litter pollutes Arctic beaches and affects wild life. Marine Pollution Bulletin 125: 535-540

Bergmann M, Tekman MB, Gutow L (2017b) Marine litter: Sea change for plastic pollution. Nature 544: 297-297

Bergmann M, Wirzberger V, Krumpen T, Lorenz C, Primpke S, Tekman MB, Gerdts G (2017c) High Quantities of Microplastic in Arctic Deep-Sea Sediments from the HAUSGARTEN Observatory. Environmental Science \& Technology 51: 11000-11010

Bergmann M, Mützel S, Primpke S, Tekman MB, Trachsel J, Gerdts G (2019) White and wonderful? Microplastics prevail in snow from the Alps to the Arctic. Science Advances 5: eaax1157 
Bessa F, Barría P, Neto JM, Frias JP, Otero V, Sobral P, Marques JC (2018) Microplastics in Juvenile Commercial Fish from an Estuarine Environment, Proceedings of the International Conference on Microplastic Pollution in the Mediterranean Sea, pp 131-135

Besseling E, Foekema EM, Van Franeker JA, Leopold MF, Kühn S, Bravo Rebolledo EL, Heße E, Mielke L, IJzer J, Kamminga P (2015) Microplastic in a macro filter feeder: Humpback whale Megaptera novaeangliae. Marine Pollution Bulletin 95: 248-252

BirdLife International (2019) Species factsheet: Fulmarus glacialis, Downloaded from http://www.birdlife.org

Bjorndal KA, Bolten AB, Lagueux CJ (1994) Ingestion of Marine Debris by Juvenile Sea Turtles in Coastal Florida Habitats. Marine Pollution Bulletin 28: 154-158

Blais JM, Kimpe LE, McMahon D, Keatley BE, Mallory ML, Douglas MS, Smol JP (2005) Arctic seabirds transport marine-derived contaminants. Science 309: 445-445

Blier PU, Dutil JD, Lemieux H, Bélanger F, Bitetera L (2007) Phenotypic flexibility of digestive system in Atlantic cod (Gadus morhua). Comparative Biochemistry and Physiology Part A: Molecular \& Integrative Physiology 146: 174-179

Boerger CM, Lattin GL, Moore SL, Moore CJ (2010) Plastic ingestion by planktivorous fishes in the North Pacific Central Gyre. Marine Pollution Bulletin 60: 2275-2278

Bogomolni AL, Pugliares KR, Sharp SM, Patchett K, Harry CT, LaRocque JM, Touhey KM, Moore MJ (2010) Mortality trends of stranded marine mammals on Cape Cod and southeastern Massachusetts, USA, 2000 to 2006. Diseases of Aquatic Organisms 88: 143-155

Bond AL, Jones IL, Williams JC, Byrd GV (2010) Auklet (Charadriiformes: Alcidae, Aethia spp.) chick meals from the Aleutian Islands, Alaska, have a very low incidence of plastic marine debris. Marine Pollution Bulletin 60: 1346-1349

Bond AL, Provencher JF, Daoust PY, Lucas ZN (2014) Plastic ingestion by fulmars and shearwaters at Sable Island, Nova Scotia, Canada. Marine Pollution Bulletin 87: 68-75

Booth A (2017) PLASTOX - Direct and indirect ecotoxicological impacts of microplastics on marine organisms. In: Joint Program Initiative (JPI) Oceans (ed)

Booth A, Kubowicz S, Beegle-Krause C, Skancke J, Nordam T, Landsem E, Throne-Holst M, Jahren S (2017) Microplastic in Global and Norwegian Marine Environments: Distributions, Degradation Mechanisms and Transport. Miljødirektoratet M-918

Botterell ZLR, Beaumont N, Dorrington T, Steinke M, Thompson RC, Lindeque PK (2019) Bioavailability and effects of microplastics on marine zooplankton: A review. Environmental Pollution 245: 98-110

Bour A, Avio CG, Gorbi S, Regoli F, Hylland K (2018) Presence of microplastics in benthic and epibenthic organisms: Influence of habitat, feeding mode and trophic level. Environmental Pollution 243: $1217-1225$

Bowers AB, Lee JW (1971) The Growth of Plaice in Laxey Bay (Isle of Man). ICES Journal of Marine Science 34: 43-50

Brandão ML, Braga KM, Luque JL (2011) Marine debris ingestion by Magellanic penguins, Spheniscus magellanicus (Aves: Sphenisciformes), from the Brazilian coastal zone. Marine Pollution Bulletin 62: 2246-2249

Bråte ILN, Eidsvoll DP, Steindal CC, Thomas KV (2016) Plastic ingestion by Atlantic cod (Gadus morhua) from the Norwegian coast. Marine Pollution Bulletin 112: 105-110

Bravo Rebolledo EL (2011) Threshold Levels and Size Dependent Passage of Plastic Litter in Stomachs of Fulmars. MSc thesis. Aquatic Ecology and Water Quality Management group, Wageningen, The Netherlands

Bravo Rebolledo EL, Van Franeker JA, Jansen OE, Brasseur SM (2013) Plastic ingestion by harbour seals (Phoca vitulina) in The Netherlands. Marine Pollution Bulletin 67: 200-202

Bray L, Digka N, Tsangaris C, Camedda A, Gambaiani D, de Lucia GA, Matiddi M, Miaud C, Palazzo L, Pérez-del-Olmo A, Raga JA, Silvestri C, Kaberi H (2019) Determining suitable fish to monitor plastic ingestion trends in the Mediterranean Sea. Environmental Pollution 247: 1071-1077 
Brennecke D, Duarte B, Paiva F, Caçador I, Canning-Clode J (2016) Microplastics as vector for heavy metal contamination from the marine environment. Estuarine, Coastal and Shelf Science 178: 189-195

Brigham L (2011) Marine protection in the Arctic cannot wait. Nature News 478: 157-157

Browne MA, Dissanayake A, Galloway TS, Lowe DM, Thompson RC (2008) Ingested Microscopic Plastic Translocates to the Circulatory System of the Mussel, Mytilus edulis (L.). Environmental Science \& Technology 42: 5026-5031

Browne MA, Crump P, Niven SJ, Teuten E, Tonkin A, Galloway T, Thompson R (2011) Accumulation of microplastic on shorelines woldwide: sources and sinks. Environmental Science \& Technology 45: 9175-9179

Browne MA, Underwood A, Chapman M, Williams R, Thompson RC, van Franeker JA (2015) Linking effects of anthropogenic debris to ecological impacts. Proceedings of the Royal Society of London B: Biological Sciences 282: 20142929

Budimir S, Setälä O, Lehtiniemi M (2018) Effective and easy to use extraction method shows low numbers of microplastics in offshore planktivorous fish from the northern Baltic Sea. Marine Pollution Bulletin 127: 586-592

Buhl-Mortensen L, Buhl-Mortensen P (2017) Marine litter in the Nordic Seas: distribution composition and abundance. Marine Pollution Bulletin 125: 260-270

Byrkjedal I, Langhelle G (2019) Selective feeding on jellyfish organs by Northern Fulmars Fulmarus glacialis. Ornis Norvegica 42: 15-18

\section{C}

Cambridge Polymer Group (2016) Rubber Duck Deformulation. Cambridge Polymer Group Inc., Boston, MA, pp 13

Camedda A, Marra S, Matiddi M, Massaro G, Coppa S, Perilli A, Ruiu A, Briguglio P, de Lucia GA (2014) Interaction between loggerhead sea turtles (Caretta caretta) and marine litter in Sardinia (Western Mediterranean Sea). Marine Environmental Research 100: 25-32

Camphuysen C, Calvo B, Durinck J, Ensor K, Follestad A, Furness R, Garthe S, Leaper G, Skov H, Tasker M (1995) Consumption of discards by seabirds in the North Sea

Camphuysen CJ, Van Franeker JA (1997) Notes on the diet of northern fulmars Fulmarus glacialis from Bjornoya (Bear Island). Sula 11: 1-10

Camphuysen CJ, Henderson JR, with major contribution from, Velilla E, Kühn S, Leopold MF, Somes R (2017) North Sea Fish and their remains. Royal Netherlands Institute of Sea Research \& Pisces Conservation Ltd, Texel, The Netherlands

Camphuysen K, Garthe S (1997) An evaluation of the distribution and scavenging habits of northern fulmars (Fulmarus glacialis) in the North Sea. ICES Journal of Marine Science 54: 654-683

Cannon SME, Lavers JL, Figueiredo B (2016) Plastic ingestion by fish in the Southern Hemisphere: A baseline study and review of methods. Marine Pollution Bulletin 107: 286-291

Carboneras C (1992) Family Procellariidae (petrels and shearwaters). Handbook of the Birds of the World 1: 216-257

Carreras-Colom E, Constenla M, Soler-Membrives A, Cartes JE, Baeza M, Padrós F, Carrassón M (2018) Spatial occurrence and effects of microplastic ingestion on the deep-water shrimp Aristeus antennatus. Marine Pollution Bulletin 133: 44-52

Chen Q, Reisser J, Cunsolo S, Kwadijk C, Kotterman M, Proietti M, Slat B, Ferrari FF, Schwarz A, Levivier A (2018) Pollutants in Plastics within the North Pacific Subtropical Gyre. Environmental Science \& Technology 52: 446-456

Chen X, Xu S, Tan T, Lee ST, Cheng SH, Lee FWF, Xu SJL, Ho KC (2014) Toxicity and estrogenic endocrine disrupting activity of phthalates and their mixtures. International Journal of Environmental Research and Public Health 11: 3156-3168

Cherel Y, Ridoux V, Weimerskirch H, Tveraa T, Chastel O (2001) Capelin (Mallotus villosus) as an important food source for northern fulmars (Fulmarus glacialis) breeding at Bjørnøya (Bear Island), Barents Sea. ICES Journal of Marine Science 58: 355-361 
Cincinelli A, Scopetani C, Chelazzi D, Lombardini E, Martellini T, Katsoyiannis A, Fossi MC, Corsolini S (2017) Microplastic in the surface waters of the Ross Sea (Antarctica): Occurrence, distribution and characterization by FTIR. Chemosphere 175: 391-400

Claessens M, De Meester S, Van Landuyt L, De Clerck K, Janssen CR (2011) Occurrence and distribution of microplastics in marine sediments along the Belgian coast. Marine Pollution Bulletin 62: 2199-2204

Clarke A, Prince PA (1976) The origin of stomach oil in marine birds: Analyses of the stomach oil from six species of subantarctic procellariiform birds. Journal of Experimental Marine Biology and Ecology 23: 15-30

Collard F, Gilbert B, Eppe G, Parmentier E, Das K (2015) Detection of anthropogenic particles in fish stomachs: an isolation method adapted to identification by Raman spectroscopy. Archives of Environmental Contamination and Toxicology 69: 331-339

Collard F, Gilbert B, Eppe G, Roos L, Compère P, Das K, Parmentier E (2017) Morphology of the filtration apparatus of three planktivorous fishes and relation with ingested anthropogenic particles. Marine Pollution Bulletin 116: 182-191

Collicutt B, Juanes F, Dudas SE (2019) Microplastics in juvenile Chinook salmon and their nearshore environments on the east coast of Vancouver Island. Environmental Pollution 244: 135-142

Courtene-Jones W, Quinn B, Gary SF, Mogg AO, Narayanaswamy BE (2017) Microplastic pollution identified in deep-sea water and ingested by benthic invertebrates in the Rockall Trough, North Atlantic Ocean. Environmental Pollution 231: 271-280

Courtene-Jones W, Quinn B, Ewins C, Gary SF, Narayanaswamy BE (2019) Consistent microplastic ingestion by deep-sea invertebrates over the last four decades (1976-2015), a study from the North East Atlantic. Environmental Pollution 244: 503-512

Cózar A, Martí E, Duarte CM, García-de-Lomas J, van Sebille E, Ballatore TJ, Eguíluz VM, GonzálezGordillo JI, Pedrotti ML, Echevarría F, Troublè R, Irigoien X (2017) The Arctic Ocean as a dead end for floating plastics in the North Atlantic branch of the Thermohaline Circulation. Science Advances 3: e1600582

Crompton TR (2007) Additive Migration from plastics into food. A guide for analytical chemists. Smithers Rapra Technology Limited, Shawbury, UK

Cury PM, Boyd IL, Bonhommeau S, Anker-Nilssen T, Crawford RJM, Furness RW, Mills JA, Murphy EJ, Österblom H, Paleczny M, Piatt JF, Roux J-P, Shannon L, Sydeman WJ (2011) Global Seabird Response to Forage Fish Depletion-One-Third for the Birds. Science 334: 1703-1706

\section{D}

Dale JJ, Meyer CG, Clark CE (2011) The ecology of coral reef top predators in the Papahānaumokuākea Marine National Monument. Journal of Marine Biology 2011: 725602

Dantas DV, Barletta M, da Costa MF (2012) The seasonal and spatial patterns of ingestion of polyfilament nylon fragments by estuarine drums (Sciaenidae). Environmental Science and Pollution Research 19: 600-606

David C, Lange B, Krumpen T, Schaafsma F, van Franeker JA, Flores H (2015) Under-ice distribution of polar cod Boreogadus saida in the central Arctic Ocean and their association with sea-ice habitat properties. Polar Biology: 1-14

Davison P, Asch RG (2011) Plastic ingestion by mesopelagic fishes in the North Pacific Subtropical Gyre. Marine Ecology Progress Series 432: 173-180

Day RH (1980) The occurence and characteristics of plastic pollution in Alaska's marine birds. Master thesis. University of Alaska, Fairbanks, pp 120

Day RH, Wehle DHS, Coleman FC (1985) Ingestion of Plastic Pollutants by Marine Birds. Shomura RS, Yoshida, H. O., Proceedings of the Workshop on the fate and impact of marine debris, Honolulu, Hawaii, pp 344-386

de Carvalho-Souza GF, Llope M, Tinôco MS, Medeiros DV, Maia-Nogueira R, Sampaio CLS (2018) Marine litter disrupts ecological processes in reef systems. Marine Pollution Bulletin 133: 464-471 
De Silva S (1973) Food and feeding habits of the herring Clupea harengus and the sprat C. sprattus in inshore waters of the west coast of Scotland. Marine Biology 20: 282-290

De Stephanis R, Gimenez J, Carpinelli E, Gutierrez-Exposito C, Canadas A (2013) As main meal for sperm whales: plastics debris. Marine Pollution Bulletin 69: 206-214

Dehaut A, Cassone A-L, Frère L, Hermabessiere L, Himber C, Rinnert E, Rivière G, Lambert C, Soudant P, Huvet A (2016) Microplastics in seafood: Benchmark protocol for their extraction and characterization. Environmental Pollution 215: 223-233

Derraik JGB (2002) The pollution of the marine environment by plastic debris: a review. Marine Pollution Bulletin 44: 842-852

Domènech F, Aznar F, Raga J, Tomás J (2019) Two decades of monitoring in marine debris ingestion in loggerhead sea turtle, Caretta caretta, from the western Mediterranean. Environmental Pollution 244: $367-378$

Donnelly-Greenan E, Hyrenbach D, Beck J, Fitzgerald S, Nevins H, Hester M (2018) First quantification of plastic ingestion by Short-tailed Albatross Phoebastria albatrus. Marine Ornithology 46: 79-84

Donnelly-Greenan EL, Harvey JT, Nevins HM, Hester MM, Walker WA (2014) Prey and plastic ingestion of Pacific Northern Fulmars (Fulmarus glacialis rogersii) from Monterey Bay, California. Marine Pollution Bulletin 85: 214-224

Dris R, Gasperi J, Saad M, Mirande C, Tassin B (2016) Synthetic fibers in atmospheric fallout: A source of microplastics in the environment? Marine Pollution Bulletin 104: 290-293

Duffy CA, Taylor GA (2015) Predation on seabirds by large teleost fishes in northern New Zealand. Bulletin of the Auckland Museum 20: 497-500

Duguy R, Duron M, Alzieu C (1980) Observation de tortues luth (Dermochelys coriacea L.) dans les partuis charentais en 1979. Annales de la Société de Sciences Naturelles de la Charente-Maritimes 6: 681-691

\section{E}

EC (2000) Directive 2000/59/EC of the European Parliament and of the Council of 27 November 2000 on port reception facilities for ship-generated waste and cargo residues. Official Journal of the European Communities L 332, pp 81-90

EC (2018) The EU Fish Market, European Commission. Directorate General for Maritime Affairs and Fisheries, European Market Observatory for Fisheries and Aquaculture Products (EUMOFA), pp 115

Edwards EW, Quinn LR, Wakefield ED, Miller PI, Thompson PM (2013) Tracking a northern fulmar from a Scottish nesting site to the Charlie-Gibbs Fracture Zone: Evidence of linkage between coastal breeding seabirds and Mid-Atlantic Ridge feeding sites. Deep Sea Research Part II: Topical Studies in Oceanography 98: 438-444

Ellis H, Gabrielsen G (2002) Energetics of free-ranging seabirds. In: Schreiber E, Burger J (eds) Biology of Marine Birds. CRC Press, Boca Raton FL, pp 359-407

Eriksen M, Lebreton LCM, Carson HS, Thiel M, Moore CJ, Borerro JC, Galgani F, Ryan PG, Reisser J (2014) Plastic Pollution in the World's Oceans: More than 5 Trillion Plastic Pieces Weighing over 250,000 Tons Afloat at Sea. PLoS One 9: e111913

Eriksson C, Burton H (2003) Origins and Biological Accumulation of Small Plastic Particles in Fur Seals from Macquarie Island. AMBIO: A Journal of the Human Environment 32: 380-384

EUBP (2016) Frequently Asked Questions on bioplastics. European Bioplastics, Berlin, pp 21

\section{F}

FAO (2019) Species fact sheet: Gadus morhua. Food and Agriculture Organization of the United Nations, pp 6

Fierens T, Servaes K, Van Holderbeke M, Geerts L, De Henauw S, Sioen I, Vanermen G (2012) Analysis of phthalates in food products and packaging materials sold on the Belgian market. Food and Chemical Toxicology 50: 2575-2583 
Finley KJ (2001) Natural history and conservation of the Greenland whale or Bowhead, in the northwest Atlantic. Arctic 54: 55-76

Fisher J (1952) The Fulmar Collins, London

Floren HP, Shugart GW (2017) Plastic in Cassin's Auklets (Ptychoramphus aleuticus) from the 2014 stranding on the Northeast Pacific Coast. Marine Pollution Bulletin 117: 496-498

Flores H, Van Franeker JA, Siegel V, Haraldsson M, Strass V, Meesters EH, Bathmann U, Wolff WJ (2012) The association of Antarctic krill Euphausia superba with the under-ice habitat. PLoS One 7: e31775

Flores H, Castellani G, Schaafsma FL, Vortkamp M, Immerz A, Zwicker S, Van Dorssen M, Tonkes H (2016) Sea ice ecology, pelagic food web and copepod physiology- ICEFLUX/PEBCAO. The Expedition PS92 of the Research Vessel POLARSTERN to the Arctic Ocean in 2015. AlfredWegener-Institute, Peeken I, The Expedition PS92 of the Research Vessel POLARSTERN to the Arctic Ocean in 2015 Reports on Polar and Marine Research, Bremerhaven

Foekema EM, De Gruijter C, Mergia MT, van Franeker JA, Murk AJ, Koelmans AA (2013) Plastic in North Sea Fish. Environmental Science \& Technology 47: 8818-8824

Foley CJ, Feiner ZS, Malinich TD, Höök TO (2018) A meta-analysis of the effects of exposure to microplastics on fish and aquatic invertebrates. Science of The Total Environment 631-632: 550-559

Forrest AK, Hindell M (2018) Ingestion of plastic by fish destined for human consumption in remote South Pacific Islands. Australian Journal of Maritime \& Ocean Affairs 10: 81-97

Fossi MC, Baini M, Panti C, Baulch S, Fossi MC, Panti C (2018) Impacts of Marine Litter on Cetaceans: A Focus on Plastic Pollution. Marine Mammal Ecotoxicology, pp 147-184

Foster KL, Wang SW, Mackay D, Mallory ML, Blais JM (2010) Preliminary assessment of avian stomach oils: a vector of contaminants to chicks and potential for diet analysis and biomonitoring. Environmental Science \& Technology 44: 6869-6874

Fotopoulou KN, Karapanagioti HK (2012) Surface properties of beached plastic pellets. Marine Environmental Research 81: 70-77

France R (1992) Garbage in paradise. Nature 355: 504

Fries E, Dekiff JH, Willmeyer J, Nuelle M-T, Ebert M, Remy D (2013) Identification of polymer types and additives in marine microplastic particles using pyrolysis-GC/MS and scanning electron microscopy. Environmental Science: Processes \& Impacts 15: 1949-1956

Froese R, Pauly D (2019) FishBase. World Wide Web electronic publication, www.fishbase.org

Furness RW, Bryant DM (1996) Effect of Wind on Field Metabolic Rates of Breeding Northern Fulmars. Ecology 77: 1181-1188

\section{G}

Gabrielsen GW, Mehlum F, Karlsen HE (1988) Thermoregulation in four species of arctic seabirds. Journal of Comparative Physiology B 157: 703-708

Galgani F, Leaute JP, Moguedet P, Souplet A, Verin Y, Carpentier A, Goraguer H, Latrouite D, Andral B, Cadiou Y, Mahe JC, Poulard JC, Nerisson P (2000) Litter on the Sea Floor Along European Coasts. Marine Pollution Bulletin 40: 516-527

Galgani F, Fleet D, Van Franeker J, Katsanevakis S, Maes T, Mouat J, Oosterbaan L, Poitou I, Hanke G, Thompson R, Amato E, Birkun A, Janssen C (2010) Marine Strategy Framework directive-Task Group 10 Report marine litter do not cause harm to the coastal and marine environment. Report on the identification of descriptors for the Good Environmental Status of European Seas regarding marine litter under the Marine Strategy Framework Directive. Office for Official Publications of the European Communities, Luxembourg

Galgani F, Hanke G, Werner S, Oosterbaan L, Nilsson P, Fleet D (2013a) Monitoring guidance for marine litter in European Seas, JRC Scientific and Policy reports, pp 120

Galgani F, Hanke G, Werner S, Oosterbaan L, Nilsson P, Fleet D, Kinsey S, Thompson RC, Van Franeker J, Vlachogianni T (2013b) Guidance on monitoring of marine litter in European Seas. A guidance 
document within the Common Implementation Strategy for the Marine Strategy Framework Directive. Publications Office of the European Union, MSFD Technical Subgroup on Marine Litter, 9279327100, Luxembourg, pp 128

Galgani F, Hanke G, Maes T (2015) Global distribution, composition and abundance of marine litter. In: Bergmann M, Gutow L, Klages M (eds) Marine Anthropogenic Litter. Springer, pp 29-56

Gall SC, Thompson RC (2015) The impact of debris on marine life. Marine Pollution Bulletin 92: 170-179

Garthe S, Montevecchi WA, Ojowski U, Stenhouse IJ (2004) Diets of northern fulmar (Fulmarus glacialis) chicks in the northwest Atlantic Ocean. Polar Biology 27: 277-280

GESAMP (2016) Sources, fate and effects of microplastics in the marine environment: part two of a global assessment, Kershaw PJ, Rochman CM, GESAMP Reports and Studies, pp 220

Geueke B, Muncke J (2017) Substances of Very High Concern in Food Contact Materials: Migration and Regulatory Background. Packaging Technology and Science 31: 757-769

Gewert B, Plassmann MM, MacLeod M (2015) Pathways for degradation of plastic polymers floating in the marine environment. Environmental Science: Processes \& Impacts 17: 1513-1521

Geyer R, Jambeck JR, Law KL (2017) Production, use, and fate of all plastics ever made. Science Advances 3: e1700782

Giani D, Baini M, Galli M, Casini S, Fossi MC (2019) Microplastics occurrence in edible fish species (Mullus barbatus and Merluccius merluccius) collected in three different geographical sub-areas of the Mediterranean Sea. Marine Pollution Bulletin 140: 129-137

Gjertz I, Mehlum F, Gabrielsen GW (1985) Food sample analysis of seabirds collected during the 'Lance'-cruise in ice-filled waters in eastern Svalbard 1984. Norwegian polar Institute, Rapportserie, Oslo, Norway, pp 21

Goldstein MC, Goodwin DS (2013) Gooseneck barnacles (Lepas spp.) ingest microplastic debris in the North Pacific Subtropical Gyre. PeerJ 1: e184

Good TP, June JA, Etnier MA, Broadhurst G (2010) Derelict fishing nets in Puget Sound and the Northwest Straits: Patterns and threats to marine fauna. Marine Pollution Bulletin 60: 39-50

Gouin T, Roche N, Lohmann R, Hodges G (2011) A Thermodynamic Approach for Assessing the Environmental Exposure of Chemicals Absorbed to Microplastic. Environmental Science \& Technology 45: 1466-1472

Gould P, Ostrom P, Walker W (1997) Trophic relationships of albatrosses associated with squid and large-mesh drift-net fisheries in the North Pacific Ocean. Canadian Journal of Zoology 75: 549-562

Gradinger RR, Bluhm BA (2004) In-situ observations on the distribution and behavior of amphipods and Arctic cod (Boreogadus saida) under the sea ice of the High Arctic Canada Basin. Polar Biology 27: 595-603

Gray H, Lattin GL, Moore CJ (2012) Incidence, mass and variety of plastics ingested by Laysan (Phoebastria immutabilis) and Black-footed Albatrosses (P. nigripes) recovered as by-catch in the North Pacific Ocean. Marine Pollution Bulletin 64: 2190-2192

Gremillet D, Prudor A, le Maho Y, Weimerskirch H (2012) Vultures of the seas: hyperacidic stomachs in wandering albatrosses as an adaptation to dispersed food resources, including fishery wastes. PLoS One 7: e37834

Gudger EW, Hoffman WH (1931) A shark encircled with a Rubber Automobile Tire. The Scientific Monthly 33: 275-277

Gudger EW (1938) The fish in the iron mask. The Scientific Monthly 46: 281-285

Gudger EW (1949) Natural history notes on tiger sharks, Galeocerdo tigrinus, caught at Key West, Florida, with emphasis on food and feeding habits. Copeia 1949: 39-47

Gutow L, Ricker M, Holstein JM, Dannheim J, Stanev EV, Wolff J-O (2018) Distribution and trajectories of floating and benthic marine macrolitter in the south-eastern North Sea. Marine Pollution Bulletin 131: 763-772 


\section{$\mathbf{H}$}

Halpern BS, Walbridge S, Selkoe KA, Kappel CV, Micheli F, D'agrosa C, Bruno JF, Casey KS, Ebert C, Fox HE (2008) A global map of human impact on marine ecosystems. Science 319: 948-952

Hamer KC, Thompson DR, Gray CM (1997) Spatial variation in the feeding ecology, foraging ranges, and breeding energetics of northern fulmars in the north-east Atlantic Ocean. ICES Journal of Marine Science 54: 645-653

HBW and BirdLife International (2018) Handbook of the Birds of the World and BirdLife International digital checklist of the birds of the world. Version 3.

Heesen HJL, Daan N, Ellis JR (2015) Fish Atlas of the Celtic Sea, North Sea and Baltic Sea. Wageningen Academic Publishers \& KNNV Publishing, Wageningen, The Netherlands

Hermabessiere L, Dehaut A, Paul-Pont I, Lacroix C, Jezequel R, Soudant P, Duflos G (2017) Occurrence and effects of plastic additives on marine environments and organisms: a review. Chemosphere

Hermsen E, Pompe R, Besseling E, Koelmans AA (2017) Detection of low numbers of microplastics in North Sea fish using strict quality assurance criteria. Marine Pollution Bulletin 122: 253-258

Hermsen E, Mintenig SM, Besseling E, Koelmans AA (2018) Quality Criteria for the Analysis of Microplastic in Biota Samples: A Critical Review. Environmental Science \& Technology 52: 10230-10240

Herzke D, Anker-Nilssen T, Nøst TH, Götsch A, Christensen-Dalsgaard S, Langset M, Fangel K, Koelmans AA (2016) Negligible Impact of ingested microplastics on tissue concentrations of persistent organic pollutants in Northern Fulmars off coastal Norway. Environmental Science \& Technology 50: 1924-1933

Hidalgo-Ruz V, Gutow L, Thompson RC, Thiel M (2012) Microplastics in the Marine Environment: A Review of the Methods Used for Identification and Quantification. Environmental Science \& Technology 46: 3060-3075

Hipfner J, Studholme K, Galbraith M (2017) Low incidence of plastics in food loads delivered to nestlings by a zooplanktivorous seabird over a 21-year period. Marine Pollution Bulletin 121: 320-322

Hipfner JM, Galbraith M, Tucker S, Studholme KR, Domalik AD, Pearson SF, Good TP, Ross PS, Hodum P (2018) Two forage fishes as potential conduits for the vertical transfer of microfibres in Northeastern Pacific Ocean food webs. Environmental Pollution 239: 215-222

Holst JC, McDonald A (2000) FISH-LIFT: a device for sampling live fish with trawls. Fisheries Research 48: 87-91

Hop H, Gjøsæter H (2013) Polar cod (Boreogadus saida) and capelin (Mallotus villosus) as key species in marine food webs of the Arctic and the Barents Sea. Marine Biology Research 9: 878-894

Humphries GR, Huettmann F (2014) Putting models to a good use: a rapid assessment of Arctic seabird biodiversity indicates potential conflicts with shipping lanes and human activity. Diversity and Distributions 20: 478-490

\section{I}

Imber MJ (1976) The origin of petrel stomach oils: a review. The Condor 78: 366-369

Isobe A, Uchiyama-Matsumoto K, Uchida K, Tokai T (2017) Microplastics in the Southern Ocean. Marine Pollution Bulletin 114: 623-626

\section{$\mathbf{J}$}

Jabeen K, Su L, Li J, Yang D, Tong C, Mu J, Shi H (2017) Microplastics and mesoplastics in fish from coastal and fresh waters of China. Environmental Pollution 221: 141-149

Jackson K (2013) 2012 Alaska Region Marine Mammal Stranding Summary. National Marine Fisheries Service, Alaska Region, Protected Resources, Juneau, Alaska pp 7

Jacobsen JK, Massey L, Gulland F (2010) Fatal ingestion of floating net debris by two sperm whales (Physeter macrocephalus). Marine Pollution Bulletin 60: 765-767

Jagiello Z, Dylewski Ł, Tobolka M, Aguirre JI (2019) Life in a polluted world: A global review of anthropogenic materials in bird nests. Environmental Pollution 251: 717-722 
Jahnke A, Arp HPH, Escher BI, Gewert B, Gorokhova E, Kühnel D, Ogonowski M, Potthoff A, Rummel C, Schmitt-Jansen M (2017) Reducing uncertainty and confronting ignorance about the possible impacts of weathering plastic in the marine environment. Environmental Science \& Technology 4: 85-90

Jambeck JR, Geyer R, Wilcox C, Siegler TR, Perryman M, Andrady A, Narayan R, Law KL (2015) Plastic waste inputs from land into the ocean. Science 347: 768-771

Jani P, Florence A, McCarthy D (1992) Further histological evidence of the gastrointestinal absorption of polystyrene nanospheres in the rat. International Journal of Pharmaceutics 84: 245-252

Jantz LA, Morishige CL, Bruland GL, Lepczyk CA (2013) Ingestion of plastic marine debris by longnose lancetfish (Alepisaurus ferox) in the North Pacific Ocean. Marine Pollution Bulletin 69: 97-104

Jawad LA, Humborstad O-B, Fjelldal PG (2016) Record of Litter Ingestion by Cod (Gadus morhua) Collected from Masfjorden, Western Norway. International Journal of Marine Science 6: 1-4

Jensen J-K (2012) Mallemukken på Færøerne / The fulmar on the Faroe Islands, Nolsoy

\section{K}

Karami A, Golieskardi A, Choo CK, Romano N, Ho YB, Salamatinia B (2017) A high-performance protocol for extraction of microplastics in fish. Science of The Total Environment 578: 485-494

Kartar S, Abou-Seedo F, Sainsbury M (1976) Polystyrene waste in the Seventh Estuary: A progress report. Marine Pollution Bulletin 4: 144

Kedzierski M, D'Almeida M, Magueresse A, Le Grand A, Duval H, César G, Sire O, Bruzaud S, Le Tilly V (2018) Threat of plastic ageing in marine environment. Adsorption/desorption of micropollutants. Marine Pollution Bulletin 127: 684-694

Kenyon KW, Kridler E (1969) Laysan Albatrosses Swallow Indigestible Matter. The Auk 86: 339-343

Kerr RA (2012) Ice-free arctic sea may be years, not decades, away. American Association for the Advancement of Science, pp 1591

Klages NTW, Cooper J (1992) Bill morphology and diet of a filter-feeding seabird: the broad-billed prion Pachyptila vittata at South Atlantic Gough Island. Journal of Zoology 227: 385-396

Koelmans AA, Besseling E, Wegner A, Foekema EM (2013) Plastic as a carrier of POPs to aquatic organisms: a model analysis. Environmental Science \& Technology 47: 7812-7820

Koelmans AA, Besseling E, Foekema EM (2014) Leaching of plastic additives to marine organisms. Environmental Pollution 187: 49-54

Koelmans AA (2015) Modeling the role of microplastics in Bioaccumulation of organic chemicals to marine aquatic organisms. A Critical Review. In: Bergmann M, Gutow L, Klages M (eds) Marine Anthropogenic Litter. Springer, pp 309-324

Kohlbach D, Schaafsma FL, Graeve M, Lange B, David C, Peeken I, van Franeker JA, Flores H (2017) Strong linkage of polar cod (Boreogadus saida) to sea ice algae-produced carbon: evidence from stomach content, fatty acid and stable isotope analyses. Progress in Oceanography 152: 62-74

Kooi M, Nes EHv, Scheffer M, Koelmans AA (2017) Ups and Downs in the Ocean: Effects of Biofouling on Vertical Transport of Microplastics. Environmental Science \& Technology 51: 7963-7971

Kosuth M, Wattenberg EV, Mason SA, Tyree C, Morrison D (2017) Synthetic polymer contamination in global drinking water. Orb Media.

Kubota T (1990) Synthetic materials found in the stomachs of lancetfish from Suruga Bay, Central Japan. Shomura RS, Godfrey ML, Proceedings of the Second International Conference on marine debris Honolulu, Hawaii US Dep Commer, NOAA Tech Memo, NMFS NOAA-TM-NMFS-SWFSC-154 pp 710-717

Kühn S, Van Franeker JA (2012) Plastic ingestion by the northern fulmar (Fulmarus glacialis) in Iceland. Marine Pollution Bulletin 64: 1252-1254

Kühn S, Bravo Rebolledo EL, van Franeker JA (2015) Deleterious effects of litter on marine life. In: Bergmann M, Gutow L, Klages M (eds) Marine Anthropogenic Litter. Springer, pp 75-116

Kühn S, van Werven B, van Oyen A, Meijboom A, Bravo Rebolledo EL, van Franeker JA (2017) The use of potassium hydroxide $(\mathrm{KOH})$ solution as a suitable approach to isolate plastics ingested by marine organisms. Marine Pollution Bulletin 115: 86-90 
Kühn S, Schaafsma FL, van Werven B, Flores H, Bergmann M, Egelkraut-Holtus M, Tekman MB, van Franeker JA (2018a) Plastic ingestion by juvenile polar cod (Boreogadus saida) in the Arctic Ocean. Polar Biology 41: 1269-1278

Kühn S, van Oyen A, Booth AM, Meijboom A, van Franeker JA (2018b) Marine microplastic: Preparation of relevant test materials for laboratory assessment of ecosystem impacts. Chemosphere 213: 103-113

Kühn S, van Franeker JA, O’Donoghue AM, Swiers A, Starkenburg M, van Werven B, Foekema E, Hermsen E, Egelkraut-Holtus M, Lindeboom H (2020) Details of plastic ingestion and fibre contamination in North Sea fishes. Environmental Pollution 257: 113569

Kukulka T, Proskurowski G, Morét-Ferguson S, Meyer DW, Law KL (2012) The effect of wind mixing on the vertical distribution of buoyant plastic debris. Geophysical Research Letters 39: L07601

L

Laist DW (1987) Overview of the biological effects of lost and discarded plastic debris in the marine environment. Marine Pollution Bulletin 18: 319-326

Laist DW (1997) Impacts of marine debris: Entanglement of Marine Life in Marine Debris Including a Comprehensive List of Species with Entanglement and Ingestion Records. In: Coe JM, Rogers DB (eds) Marine Debris Sources, Impacts and Solutions. Springer Series on Environmental Management, New York, pp 99-132

Lavell R (1989) Nutrition and feeding of fish. Springer, Boston, MA

Lavers JL, Bond AL (2016) Ingested plastic as a route for trace metals in Laysan Albatross (Phoebastria immutabilis) and Bonin Petrel (Pterodroma hypoleuca) from Midway Atoll. Marine Pollution Bulletin 110: 493-500

Lavers JL, Bond AL (2017) Exceptional and rapid accumulation of anthropogenic debris on one of the world's most remote and pristine islands. Proceedings of the National Academy of Sciences 114: 6052-6055

Law KL, Thompson RC (2014) Microplastics in the seas. Science 345: 144-145

Leclerc L-ME, Lydersen C, Haug T, Bachmann L, Fisk AT, Kovacs KM (2012) A missing piece in the Arctic food web puzzle? Stomach contents of Greenland sharks sampled in Svalbard, Norway. Polar Biology 35: 1197-1208

Leggett CG, Scherer N, Curry MS, Bailey R, Haab TC (2014) Assessing the economic benefits of reductions in marine debris: a pilot study of beach recreation in Orange County, California. Industrial Economics, Incorporated, pp 45

Lenz R, Enders K, Beer S, Sorensen TK, Stedmon CA (2016) Analysis of microplastic in the stomachs of herring and cod from the North Sea and Baltic Sea. Technical University of Denmark. DTU Aqua. National Institute of Aquatic Resources, Lyngby, Denmark, pp 29

Lenzi J, Burgues MF, Carrizo D, Machin E, Teixeira-de Mello F (2016) Plastic ingestion by a generalist seabird on the coast of Uruguay. Marine Pollution Bulletin 107: 71-76

Leopold MF, Van Bemmelen RSA, Geelhoed SCV, Verdaat H, Bravo Rebolledo EL (2013) Futen in de Hollandse Noordzeekustzone in december 2012 en januari 2013. IMARES Wageningen UR C30/13, Texel, The Netherlands, pp 35

Lewis R (1969) Studies on the stomach oils of marine animals-II. Oils of some procellariiform birds. Comparative Biochemistry and Physiology 31: 725-731

Liboiron M, Liboiron F, Wells E, Richard N, Zahara A, Mather C, Bradshaw H, Murichi J (2016) Low plastic ingestion rate in Atlantic Cod (Gadus morhua) from Newfoundland destined for human consumption collected through citizen science methods. Marine Pollution Bulletin 113: 428-437

Liboiron M, Melvin J, Richárd N, Saturno J, Ammendolia J, Liboiron F, Charron L, Mather C (2019) Low incidence of plastic ingestion among three fish species significant for human consumption on the island of Newfoundland, Canada. Marine Pollution Bulletin 141: 244-248

Lithner D, Damberg J, Dave G, Larsson K (2009) Leachates from plastic consumer products-screening for toxicity with Daphnia magna. Chemosphere 74: 1195-1200 
Lithner D (2011) Environmental and health hazards of chemicals in plastic polymers and products. $\mathrm{PhD}$. Department of Plant and Environmental Sciences, Göteborg

Lithner D, Larsson A, Dave G (2011) Environmental and health hazard ranking and assessment of plastic polymers based on chemical composition. Science of The Total Environment 409: 33093324

Llompart M, Sanchez-Prado L, Lamas JP, Garcia-Jares C, Roca E, Dagnac T (2013) Hazardous organic chemicals in rubber recycled tire playgrounds and pavers. Chemosphere 90: 423-431

Löder MG, Gerdts G (2015) Methodology used for the detection and identification of microplastics-A critical appraisal. In: Bergmann M, Gutow L, Klages M (eds) Marine anthropogenic Litter. Springer, pp 201-227

Lønne O, Gulliksen B (1989) Size, age and diet of polar cod, Boreogadus saida (Lepechin 1773), in ice covered waters. Polar Biology 9: 187-191

Lønne O, Gabrielsen G (1992) Summer diet of seabirds feeding in sea-ice-covered waters near Svalbard. Polar Biology 12: 685-692

Lusher A, McHugh M, Thompson R (2013) Occurrence of microplastics in the gastrointestinal tract of pelagic and demersal fish from the English Channel. Marine Pollution Bulletin 67: 94-99

Lusher A (2015) Microplastics in the marine environment: distribution, interactions and effects. In: Bergmann M, Gutow L, Klages M (eds) Marine Anthropogenic Litter. Springer, Cham, pp 245-307

Lusher A, Bråte ILN, Hurley R, Iversen K, Olsen M (2017a) Testing of methodology for measuring microplastics in blue mussels (Mytilus spp) and sediments, and recommendations for future monitoring of microplastics (R \& D-project) pp 88, 0803-625X, pp 88

Lusher AL, Tirelli V, O'Connor I, Officer R (2015) Microplastics in Arctic polar waters: the first reported values of particles in surface and sub-surface samples. Scientific Reports 5: 14947

Lusher AL, O'Donnell C, Officer R, O'Connor I (2016) Microplastic interactions with North Atlantic mesopelagic fish. ICES Journal of Marine Science 73: 1214-1225

Lusher AL, Welden NA, Sobral P, Cole M (2017b) Sampling, isolating and identifying microplastics ingested by fish and invertebrates. Analytical Methods 9: 1346-1360

Lydersen C, Giertz I, Weslawski JM (1985) Aspects of vertebrate feeding in the marine ecosystem in Hornsund, Svalbard. Norsk Polarinstitutt Oslo, pp 57

Lydersen C, Gjertz I, Weslawski JM (1989) Stomach contents of autumn-feeding marine vertebrates from Hornsund, Svalbard. Polar Record 25: 107-114

\section{M}

Mallory M, Karnovsky NJ, Gaston AJ, Hobson KA, Provencher JF, Forbes MR, Hunt Jr. GL, Byers T, Dick TA (2010) Temporal and spatial patterns in the diet of northern fulmars Fulmarus glacialis in the Canadian High Arctic. Aquatic Biology 10: 181-191

Mallory ML (2006) The Northern Fulmar (Fulmarus glacialis) in Arctic Canada: ecology, threats, and what it tells us about marine environmental conditions. Environmental Reviews 14: 187-216

Mallory ML, Roberston GJ, Moenting A (2006) Marine plastic debris in northern fulmars from Davis Strait, Nunavut, Canada. Marine Pollution Bulletin 52: 813-815

Mallory ML (2008) Marine plastic debris in northern fulmars from the Canadian high Arctic. Marine Pollution Bulletin 56: 1501-1504

Mallory ML, Hatch SA, Nettleship DN (2012) Northern Fulmar (Fulmarus glacialis), version 2.0. In: Poole AF (ed) The Birds of North America Cornell Lab of Ornithology, Ithaca, NY, USA

Mark FC (2015) Station list and links to master tracks in different resolutions of HEINCKE cruise HE451-1, Troms $\varnothing$ - Longyearbyen, 2015-09-11 - 2015-09-29. PANGAEA

Markic A, Gaertner J-C, Gaertner-Mazouni N, Koelmans AA (2019) Plastic ingestion by marine fish in the wild. Critical Reviews in Environmental Science and Technology: 1-41

Martin AR, Clarke MR (1986) The diet of sperm whales (Physeter macrocephalus) captured between Iceland and Greenland. Journal of the Marine Biological Association of the United Kingdom 66: 779-790 
Mate BR (1985) Incidents of marine mammal encounters with debris in active fishing gear. Shomura RS, Yoshida HO, Proceedings of the Workshop on the fate and impact of marine debris, Honolulu, Hawaii, pp 453-457

Matiddi M, Hochsheid S, Camedda A, Baini M, Cocumelli C, Serena F, Tomassetti P, Travaglini A, Marra S, Campani T, Scholl F, Mancusi C, Amato E, Briguglio P, Maffucci F, Fossi MC, Bentivegna F, de Lucia GA (2017) Loggerhead sea turtles (Caretta caretta): A target species for monitoring litter ingested by marine organisms in the Mediterranean Sea. Environmental Pollution 230: 199-209

Mato Y, Isobe T, Takada H, Kanehiro H, Ohtake C, Kaminuma T (2001) Plastic resin pellets as a transport medium for toxic chemicals in the marine environment. Environmental Science \& Technology 35: 318-324

Mattsson K, Johnson EV, Malmendal A, Linse S, Hansson L-A, Cedervall T (2017) Brain damage and behavioural disorders in fish induced by plastic nanoparticles delivered through the food chain. Scientific Reports 7: 11452

McGoran A, Clark P, Morritt D (2017) Presence of microplastic in the digestive tracts of European flounder, Platichthys flesus, and European smelt, Osmerus eperlanus, from the River Thames. Environmental Pollution 220: 744-751

McGoran AR, Cowie PR, Clark PF, McEvoy JP, Morritt D (2018) Ingestion of plastic by fish: A comparison of Thames Estuary and Firth of Clyde populations. Marine Pollution Bulletin 137: 12-23

Mehlum F, Gabrielsen G (1993) The diet of high-arctic seabirds in coastal and ice-covered, pelagic areas near the Svalbard archipelago. Polar Research 12: 1-20

MEPC (2011) Amendments to the Annex of the Protocol of 1978 relating to the International Convention for the Prevention of Pollution from Ships, 1973 (Revised MARPOL Annex V). In: IMO (ed) Resolution MEPC201(62) IMO, London, pp 12

Miranda DdA, de Carvalho-Souza GF (2016) Are we eating plastic-ingesting fish? Marine Pollution Bulletin 103: 109-114

Mizraji R, Ahrendt C, Perez-Venegas D, Vargas J, Pulgar J, Aldana M, Ojeda FP, Duarte C, GalbánMalagón C (2017) Is the feeding type related with the content of microplastics in intertidal fish gut? Marine Pollution Bulletin 116: 498-500

Mohamed Nor NH, Koelmans AA (2019) Transfer of PCBs from Microplastics under Simulated Gut Fluid Conditions Is Biphasic and Reversible. Environmental Science \& Technology 53: 1874-1883

Möllmann C, Kornilovs G, Fetter M, Köster F (2004) Feeding ecology of central Baltic Sea herring and sprat. Journal of Fish Biology 65: 1563-1581

Moore CJ, Moore SL, Leecaster MK, Weisberg SB (2001) A Comparison of Plastic and Plankton in the North Pacific Central Gyre. Marine Pollution Bulletin 42: 1297-1300

Moore CJ (2008) Synthetic polymers in the marine environment: A rapidly increasing, long-term threat. Environmental Research 108: 131-139

Morgana S, Ghigliotti L, Estévez-Calvar N, Stifanese R, Wieckzorek A, Doyle T, Christiansen JS, Faimali M, Garaventa F (2018) Microplastics in the Arctic: A case study with sub-surface water and fish samples off Northeast Greenland. Environmental Pollution 242: 1078-1086

Moser ML, Lee DS (1992) A Fourteen-Year survey of Plastic Ingestion by Western North Atlantic Seabirds. Colonial Waterbirds 15: 83-94

Mouat J, Lozano RL, Bateson H (2010) Economic Impacts of Marine Litter. KIMO International, pp. 105

MSFD GES Technical Subgroup on Marine Litter (2011) Marine Litter - Technical Recommendations for the Implementation of MSFD Requirements, Publications Office of the European Union, Luxembourg, pp 91

Murphy F, Russell M, Ewins C, Quinn B (2017) The uptake of macroplastic \& microplastic by demersal \& pelagic fish in the Northeast Atlantic around Scotland. Marine Pollution Bulletin 122: 353-359

Murray F, Cowie PR (2011) Plastic contamination in the decapod crustacean Nephrops norvegicus (Linnaeus, 1758). Marine Pollution Bulletin 62: 1207-1217 
$\mathbf{N}$

Nadal M, Alomar C, Deudero S (2016) High levels of microplastic ingestion by the semipelagic fish bogue Boops boops (L.) around the Balearic Islands. Environmental Pollution 214: 517-523

Napper IE, Thompson RC (2016) Release of synthetic microplastic plastic fibres from domestic washing machines: Effects of fabric type and washing conditions. Marine Pollution Bulletin 112: 39-45

Nelms SE, Galloway TS, Godley BJ, Jarvis DS, Lindeque PK (2018) Investigating microplastic trophic transfer in marine top predators. Environmental Pollution 238: 999-1007

Nevins H, Hyrenbach D, Keiper C, Stock J, Hester M, Harvey J (2005) Seabirds as indicators of plastic pollution in the North Pacific, Paper for Plastic Debris Rivers to the Sea Conference, Redondo Beach, California, United States of America, pp 12

Newman S, Watkins E, Farmer A, ten Brink P, Schweitzer J-P (2015) The economics of marine litter. In: Bergmann M, Gutow L, Klages M (eds) Marine Anthropogenic Litter. Springer, pp 367-394

Nicolson A (2018) The Seabird's Cry: The Lives and Loves of the Planet's Great Ocean Voyagers. Henry Holt and Company

Nielsen J, Hedeholm RB, Simon M, Steffensen JF (2013) Distribution and feeding ecology of the Greenland shark (Somniosus microcephalus) in Greenland waters. Polar Biology 37: 37-46

North Sea Ministerial Conference (2002) Bergen Declaration. Ministerial Declaration of the Fifth International Conference on the Protection of the North Sea, Bergen, Norway, pp 50

\section{O}

O'Brine T, Thompson RC (2010) Degradation of plastic carrier bags in the marine environment. Marine Pollution Bulletin 60: 2279-2283

O'Neill M (2010) Regression \& Generalized Linear (Mixed) Models. Statistical Advisory \& Training Service Pty Ltd

Obbard RW, Sadri S, Wong YQ, Khitun AA, Baker I, Thompson RC (2014) Global warming releases microplastic legacy frozen in Arctic Sea ice. Earth's Future 2: 315-320

Obbard RW (2018) Microplastics in Polar Regions: The role of long range transport. Current Opinion in Environmental Science \& Health 1: 24-29

Ocean Conservancy (2016) International Coastal Cleanup, Annual Report, Washington, DC, pp 24

Oehlmann J, Schulte-Oehlmann U, Kloas W, Jagnytsch O, Lutz I, Kusk KO, Wollenberger L, Santos EM, Paull GC, Van Look KJ, Tyler CR (2009) A critical analysis of the biological impacts of plasticizers on wildlife. Philos Trans R Soc Lond B Biol Sci 364: 2047-2062

Ojowski U, Eidtmann C, Furness R, Garthe S (2001) Diet and nest attendance of incubating and chickrearing northern fulmars (Fulmarus glacialis) in Shetland. Marine Biology 139: 1193-1200

Ory N, Chagnon C, Felix F, Fernández C, Ferreira JL, Gallardo C, Ordóñez OG, Henostroza A, Laaz E, Mizraji R (2018) Low prevalence of microplastic contamination in planktivorous fish species from the southeast Pacific Ocean. Marine Pollution Bulletin 127: 211-216

Ory NC, Sobral P, Ferreira JL, Thiel M (2017) Amberstripe scad Decapterus muroadsi (Carangidae) fish ingest blue microplastics resembling their copepod prey along the coast of Rapa Nui (Easter Island) in the South Pacific subtropical gyre. Science of The Total Environment 586: 430-437

OSPAR (2008) Background document for the EcoQO on plastic Particles in stomachs of seabirds. OSPAR Commision, London

OSPAR (2010) The OSPAR system of Ecological Quality Objectives for the North Sea: a contribution to OSPAR's Quality Status Report 2010, OSPAR Commission \& Rijkswaterstaat VenW, London \& Rijswijk, pp 16

OSPAR (2015) Guidelines for Monitoring of plastic particles in stomachs of fulmars in the North Sea area, OSPAR Commission Agreement 2015-03, pp 26

OSPAR (2017) OSPAR Intermediate Assessment 2017. Plastic Particles in Fulmar Stomachs in the North Sea, OSPAR Assessment Portal OAP 


\section{$\mathbf{P}$}

Parton KJ, Galloway TS, Godley BJ (2019) Global review of shark and ray entanglement in anthropogenic marine debris. Endangered Species Research 39: 173-190

Payne R, Murray D, Harding S (2015) An Introduction to the Genstat Command Language (18th Edition), Hemel, Hempstead, UK

Peeken I, Primpke S, Beyer B, Gütermann J, Katlein C, Krumpen T, Bergmann M, Hehemann L, Gerdts G (2018) Arctic sea ice is an important temporal sink and means of transport for microplastic. Nature Communications 9: 1505

Pellini G, Gomiero A, Fortibuoni T, Ferrà C, Grati F, Tassetti N, Polidori P, Fabi G, Scarcella G (2018) Characterization of microplastic litter in the gastrointestinal tract of Solea solea from the Adriatic Sea. Environmental Pollution 234: 943-952

Pennycuick CJ (2008) Chapter 11 Information Systems for Flying Animals Theoretical Ecology Series. Academic Press, pp 305-331

Perry MC, Olsen GH, Richards RA, Osenton PC (2013) Predation on Dovekies by Goosefish Over Deep Water in the Northwest Atlantic Ocean. Northeastern Naturalist 20: 148-154

Pettit TN, Grant GS, Whittow GC (1981) Ingestion of plastics by Laysan Albatross. The Auk 98: 839-841

Pham CK, Rodríguez Y, Dauphin A, Carriço R, Frias JP, Vandeperre F, Otero V, Santos MR, Martins HR, Bolten AB (2017) Plastic ingestion in oceanic-stage loggerhead sea turtles (Caretta caretta) off the North Atlantic subtropical gyre. Marine Pollution Bulletin 121: 222-229

Phillips R, Petersen M, Lilliendahl K, Solmundsson J, Hamer K, Camphuysen C, Zonfrillo B (1999) Diet of the northern fulmar Fulmarus glacialis: reliance on commercial fisheries? Marine Biology 135: 159-170

Phuong NN, Zalouk-Vergnoux A, Poirier L, Kamari A, Châtel A, Mouneyrac C, Lagarde F (2016) Is there any consistency between the microplastics found in the field and those used in laboratory experiments? Environmental Pollution 211: 111-123

Place AR, Stoyan NC, Ricklefs RE, Butler RG (1989) Physiological Basis of Stomach Oil Formation in Leach's Storm-Petrel (Oceanodroma leucorhoa). The Auk 106: 687-699

PlasticsEurope (2011) Plastics - the Facts 2011. An analysis of European plastics production, demand and recovery for 2010 Manufacturers PAoP, Brussels, pp 32 www.plasticseurope.org

PlasticsEurope (2013) Plastics-the Facts 2013. An analysis of European latest plastics production, demand and waste data, PlasticsEurope Association of Plastics Manufacturers, Brusseles, pp 29 www.plasticseurope.org

PlasticsEurope (2018) Plastics - the Facts 2017. An analysis of European latest plastics production, demand and waste data, Wemmel, Belgium, pp 60 www.plasticseurope.org

Poon FE, Provencher JF, Mallory ML, Braune BM, Smith PA (2016) Levels of ingested debris vary across species in Canadian Arctic seabirds. Marine Pollution Bulletin 116: 517-520

Pozo K, Gomez V, Torres M, Vera L, Nuñez D, Oyarzún P, Mendoza G, Clarke B, Fossi MC, Baini M (2019) Presence and characterization of microplastics in fish of commercial importance from the Biobío region in central Chile. Marine Pollution Bulletin 140: 315-319

Prince PA (1980) The food and feeding ecology of Blue petrel (Halobaena caerulea) and Dove prion (Pachyptila desolata). Journal of Zoology 190: 59-76

Prokhorova T, Krivosheya P (2013) Monitoring the marine environment - Anthropogenic matter. IMR/PINRO Joint Report Series, Prokhorova T, Survey report from the joint Norwegian/Russian ecosystem Survey in the Barents Sea and adjacent waters, August-October 2013, 1502-8828, Murmansk, pp 131

Provencher JF, Gaston AJ, Mallory M (2009) Evidence for increased ingestion of plastics by northern fulmars (Fulmarus glacialis) in the Canadian Arctic. Marine Pollution Bulletin 58: 1078-1096

Provencher JF, Gaston AJ, Mallory M (2010) Seabirds and plastics: Sentinel species in the Canadian Arctic, AGU Ocean Sciences Meeting Portland, OR (USA)

Provencher JF, Bond AL, Avery-Gomm S, Borrelle SB, Bravo Rebolledo EL, Hammer S, Kühn S, Lavers JL, Mallory ML, Trevail A, van Franeker JA (2017) Quantifying ingested debris in marine megafauna: a review and recommendations for standardization. Analytical Methods 9: 1454-1469 
Provencher JF, Avery-Gomm S, Liboiron M, Braune BM, Macaulay JB, Mallory ML, Letcher RJ (2018) Are ingested plastics a vector of PCB contamination in northern fulmars from coastal Newfoundland and Labrador? Environmental Research 167: 184-190

Provencher JF, Borrelle SB, Bond AL, Lavers JL, Van Franeker JA, Kühn S, Hammer S, Avery-Gomm S, Mallory ML (2019) Recommended best practices for plastic and litter ingestion studies in marine birds: Collection, processing, and reporting. Facets 4: 111-130

Provencher JF, Avery-Gomm S, Braune BM, Letcher RJ, Dey CJ, Mallory ML (2020) Are phthalate ester contaminants in northern fulmar preen oil higher in birds that have ingested more plastic? Marine Pollution Bulletin 150: 110679

Puls J, Wilson SA, Hölter D (2011) Degradation of cellulose acetate-based materials: a review. Journal of Polymers and the Environment 19: 152-165

\section{$\mathbf{R}$}

R Core Team (2014) R: a language and environment for statistical computing In: R Foundation for Statistical Computing (ed), Vienna, Austria, http://www.R-project.org/

Rani M, Shim WJ, Han GM, Jang M, Al-Odaini NA, Song YK, Hong SH (2015) Qualitative analysis of additives in plastic marine debris and its new products. Archives of Environmental Contamination and Toxicology 69: 352-366

Rapp DC, Youngren SM, Hartzell P, Hyrenbach KD (2017) Community-wide patterns of plastic ingestion in seabirds breeding at French Frigate Shoals, Northwestern Hawaiian Islands. Marine Pollution Bulletin

Riotte-Lambert L, Weimerskirch H (2013) Do naive juvenile seabirds forage differently from adults? Proceedings of the Royal Society B: Biological Sciences 280: 20131434

Rizzi M, Rodrigues FL, Medeiros L, Ortega I, Rodrigues L, Monteiro DS, Kessler F, Proietti MC (2019) Ingestion of plastic marine litter by sea turtles in southern Brazil: abundance, characteristics and potential selectivity. Marine Pollution Bulletin 140: 536-548

Rochman CM, Hoh E, Hentschel BT, Kaye S (2012) Long-Term Field Measurement of Sorption of Organic Contaminants to Five Types of Plastic Pellets: Implications for Plastic Marine Debris. Environmental Science \& Technology 47: 1646-1654

Rochman CM, Browne MA, Halpern BS, Hentschel BT, Hoh E, Karapanagioti HK, Rios-Mendoza LM, Takada H, Teh S, Thompson RC (2013) Policy: Classify plastic waste as hazardous. Nature 494: 169-171

Rochman CM, Hentschel BT, Teh SJ (2014) Long-term sorption of metals is similar among plastic types: implications for plastic debris in aquatic environments. PLoS One 9: e85433

Rochman CM (2015) The complex mixture, fate and toxicity of chemicals associated with plastic debris in the marine environment. In: Bergmann M, Gutow L, Klages M (eds) Marine Anthropogenic Litter. Springer, pp 117-140

Rochman CM, Tahir A, Williams SL, Baxa DV, Lam R, Miller JT, Teh F-C, Werorilangi S, Teh SJ (2015) Anthropogenic debris in seafood: Plastic debris and fibers from textiles in fish and bivalves sold for human consumption. Scientific Reports 5: 14340

Rochman CM, Browne MA, Underwood AJ, van Franeker JA, Thompson Richard C, Amaral-Zettler LA (2016) The ecological impacts of marine debris: unraveling the demonstrated evidence from what is perceived. Ecology 97: 302-312

Rodríguez A, Rodríguez B, Nazaret Carrasco M (2012) High prevalence of parental delivery of plastic debris in Cory's shearwaters (Calonectris diomedea). Marine Pollution Bulletin 64: 2219-2223

Rodríguez A, Ramírez F, Carrasco MN, Chiaradia A (2018) Seabird plastic ingestion differs among collection methods: Examples from the short-tailed shearwater. Environmental Pollution 243: 1750-1757

Roman L, Schuyler QA, Hardesty BD, Townsend KA (2016) Anthropogenic Debris Ingestion by Avifauna in Eastern Australia. PLoS One 11: e0158343

Roman L, Hardesty BD, Hindell MA, Wilcox C (2019a) A quantitative analysis linking seabird mortality and marine debris ingestion. Scientific Reports 9: 3202 
Roman L, Lowenstine L, Parsley LM, Wilcox C, Hardesty BD, Gilardi K, Hindell M (2019b) Is plastic ingestion in birds as toxic as we think? Insights from a plastic feeding experiment. Science of The Total Environment 665: 660-667

Roman L, Paterson H, Townsend KA, Wilcox C, Hardesty BD, Hindell MA (2019c) Size of marine debris items ingested and retained by petrels. Marine Pollution Bulletin 142: 569-575

Rothstein SI (1973) Plastic particle pollution of the surface of the Atlantic Ocean: evidence from a seabird. The Condor 75: 344-345

Rummel CD, Löder MG, Fricke NF, Lang T, Griebeler E-M, Janke M, Gerdts G (2016) Plastic ingestion by pelagic and demersal fish from the North Sea and Baltic Sea. Marine Pollution Bulletin 102: 134-141

Ryan PG (1987) The incidence and characteristics of plastic particles ingested by seabirds. Marine Environmental Research 23: 175-206

Ryan PG, Jackson S (1987) The lifespan of ingested plastic particles in seabirds and their effect on digestive efficiency. Marine Pollution Bulletin 18: 217-219

Ryan PG (1988) Effects of ingested plastic on seabird feeding: evidence from chickens. Marine Pollution Bulletin 19: 125-128

Ryan PG (2008) Seabirds indicate changes in the composition of plastic litter in the Atlantic and southwestern Indian Oceans. Marine Pollution Bulletin 56: 1406-1409

Ryan PG, Moore CJ, van Franeker JA, Moloney CL (2009) Monitoring the abundance of plastic debris in the marine environment. Philos Trans R Soc Lond B Biol Sci 364: 1999-2012

Ryan PG (2015a) How quickly do albatrosses and petrels digest plastic particles? Environmental Pollution 207: 438-440

Ryan PG (2015b) A brief history of marine litter research. In: Bergmann M, Gutow L, Klages M (eds) Marine anthropogenic Litter. Springer, Cham, pp 1-25

Ryan PG (2018) Entanglement of birds in plastics and other synthetic materials. Marine Pollution Bulletin 135: 159-164

\section{$S$}

Sadri SS, Thompson RC (2014) On the quantity and composition of floating plastic debris entering and leaving the Tamar Estuary, Southwest England. Marine Pollution Bulletin 81: 55-60

SAPEA (2019) A Scientific Perspective on Microplastics in Nature and Society, Science Advice for Policy by European Academies, Berlin, Germany, pp 176

Schecter A, Lorber M, Guo Y, Wu Q, Yun SH, Kannan K, Hommel M, Imran N, Hynan LS, Cheng D (2013) Phthalate concentrations and dietary exposure from food purchased in New York State. Environmental Health Perspectives 121: 473

Schreiber EA, Burger J (2001) Biology of marine birds. CRC press

Schulz M, Bergmann M, von Juterzenka K, Soltwedel T (2010) Colonisation of hard substrata along a channel system in the deep Greenland Sea. Polar Biology 33: 1359-1369

Schulz M, Neumann D, Fleet DM, Matthies M (2013) A multi-criteria evaluation system for marine litter pollution based on statistical analyses of OSPAR beach litter monitoring time series. Marine Environmental Research 92: 61-70

Schuyler Q, Hardesty BD, Wilcox C, Townsend K (2012) To Eat or Not to Eat? Debris Selectivity by Marine Turtles. PLoS One 7: e40884

Schuyler Q, Hardesty BD, Wilcox C, Townsend K (2014) Global analysis of anthropogenic debris ingestion by sea turtles. Conservation Biology 28: 129-139

Schwalme K, Chouinard GA (1999) Seasonal dynamics in feeding, organ weights, and reproductive maturation of Atlantic cod (Gadus morhua) in the southern Gulf of St Lawrence. ICES Journal of Marine Science 56: 303-319

Scordino J (1985) Studies on fur seal entanglement, 1981-84, St Paul Island, Alaska. Shomura RS, Yoshida HO, Proceedings of the Workshop on the Fate and Impact of Marine Debris, Honolulu, Hawaii, pp 278-290 
Serreze MC, Holland MM, Stroeve J (2007) Perspectives on the Arctic's shrinking sea-ice cover. Science 315: 1533-1536

Shah AA, Hasan F, Shah Z, Kanwal N, Zeb S (2013) Biodegradation of natural and synthetic rubbers: a review. International Biodeterioration \& Biodegradation 83: 145-157

Sileo L, Sievert PR, Samuel MD, Fefer SI (1990) Prevalence and characteristics of plastic ingested by Hawaiian seabirds. Shomura RS, Godfrey ML, Proceedings of the second international Conference on marine debris, 2-7 April 1989, Honolulu, Hawaii, pp 665-681

Singh B, Sharma N (2008) Mechanistic implications of plastic degradation. Polymer Degradation and Stability 93: 561-584

Slaughter E, Gersberg RM, Watanabe K, Rudolph J, Stransky C, Novotny TE (2011) Toxicity of cigarette butts, and their chemical components, to marine and freshwater fish. Tobacco Control 20: i25-i29

Smith LC, Stephenson SR (2013) New Trans-Arctic shipping routes navigable by midcentury. Proceedings of the National Academy of Sciences 110: E1191-E1195

Society for Marine Mammalogy (2018) List of marine mammal species and subspecies Committee on Taxonomy, www.marinemammalscience.org

Sommers J (2014) Dildo Found in Cod's Stomach by Norwegian Fisherman Huffington Post. Verizon media

Spear LB, Ainley DG, Ribic CA (1995) Incidence of Plastic in Seabirds from the Tropical Pacific 1984-91: Relation with Distribution of species, Sex, Age, Season, Year and Body Weight. Marine Environmental Research 40: 123-146

Spreen G, Kwok R, Menemenlis D (2011) Trends in Arctic sea ice drift and role of wind forcing: 1992-2009. Geophysical Research Letters 38: L19501

Staffieri E, de Lucia GA, Camedda A, Poeta G, Battisti C (2019) Pressure and impact of anthropogenic litter on marine and estuarine reptiles: an updated "blacklist" highlighting gaps of evidence. Environmental Science and Pollution Research 26: 1238-1249

Steer M, Cole M, Thompson RC, Lindeque PK (2017) Microplastic ingestion in fish larvae in the western English Channel. Environmental Pollution 226: 250-259

Stelfox M, Hudgins J (2015) A two year summary of turtle entanglements in ghost gear in the Maldives. Indian Ocean Turtle Newsletter 22: 1-7

Suhrhoff TJ, Scholz-Böttcher BM (2016) Qualitative impact of salinity, UV radiation and turbulence on leaching of organic plastic additives from four common plastics-A lab experiment. Marine Pollution Bulletin 102: 84-94

Sun C, Soltwedel T, Bauerfeind E, Adelman DA, Lohmann R (2016) Depth profiles of persistent organic pollutants in the North and Tropical Atlantic Ocean. Environmental Science \& Technology 50: 6172-6179

Swennen C (1974) Observations on the effect of ejection of stomach oil by the fulmar Fulmarus glacialis on other birds. Ardea 62: 111-117

\section{T}

Tahir A, Rochman CM (2015) Plastic Particles in Silverside (Stolephorus heterolobus) Collected at Paotere Fish Market, Makassar. International Journal of Agriculture System 2: 163-168

Tanabe S, Watanabe M, Minh TB, Kunisue T, Nakanishi S, Ono H, Tanaka H (2003) PCDDs, PCDFs, and Coplanar PCBs in Albatross from the North Pacific and Southern Oceans: Levels, Patterns, and Toxicological Implications. Environmental Science \& Technology 38: 403-413

Tanaka K, Takada H, Yamashita R, Mizukawa K, Fukuwaka M-a, Watanuki Y (2013) Accumulation of plastic-derived chemicals in tissues of seabirds ingesting marine plastics. Marine Pollution Bulletin 69: 219-222

Tanaka K, Takada H, Yamashita R, Mizukawa K, Fukuwaka M-a, Watanuki Y (2015) Facilitated leaching of additive-derived PBDEs from plastic by seabirds' stomach oil and accumulation in tissues. Environmental Science \& Technology 49: 11799-11807 
Tanaka K, Takada H (2016) Microplastic fragments and microbeads in digestive tracts of planktivorous fish from urban coastal waters. Scientific Reports 6: 34351

Tanaka K, van Franeker JA, Deguchi T, Takada H (2019) Piece-by-piece analysis of additives and manufacturing byproducts in plastics ingested by seabirds: Implication for risk of exposure to seabirds. Marine Pollution Bulletin 145: 36-41

Tekman MB, Krumpen T, Bergmann M (2017) Marine litter on deep Arctic seafloor continues to increase and spreads to the North at the HAUSGARTEN observatory. Deep Sea Research Part I: Oceanographic Research Papers 120: 88-99

Ter Halle A, Ladirat L, Gendre X, Goudounèche D, Pusineri C, Routaboul C, Tenailleau C, Duployer B, Perez E (2016) Understanding the fragmentation pattern of marine plastic debris. Environmental Science \& Technology 50: 5668-5675

Terepocki AK, Brush AT, Kleine LU, Shugart GW, Hodum P (2017) Size and dynamics of microplastic in gastrointestinal tracts of Northern Fulmars (Fulmarus glacialis) and Sooty Shearwaters (Ardenna grisea). Marine Pollution Bulletin 116: 143-150

Teuten EL, Saquing JM, Knappe DR, Barlaz MA, Jonsson S, Bjorn A, Rowland SJ, Thompson RC, Galloway TS, Yamashita R, Ochi D, Watanuki Y, Moore C, Viet PH, Tana TS, Prudente M, Boonyatumanond R, Zakaria MP, Akkhavong K, Ogata Y, Hirai H, Iwasa S, Mizukawa K, Hagino Y, Imamura A, Saha M, Takada H (2009) Transport and release of chemicals from plastics to the environment and to wildlife. Philos Trans R Soc Lond B Biol Sci 364: 2027-2045

Thiel M, Hinojosa I, Vásquez N, Macaya E (2013) Floating marine debris in coastal waters of the SEPacific (Chile). Marine Pollution Bulletin 46: 224-231

Thompson DR, Furness RW, Lewis SA (1995) Diets and long-term changes in $\delta 15 \mathrm{~N}$ and $\delta 13 \mathrm{C}$ values in northern fulmars Fulmarus glacialis from two northeast Atlantic colonies. Marine Ecology Progress Series 125: 3-11

Thompson RC, Moore CJ, vom Saal FS, Swan SH (2009) Plastics, the environment and human health: current consensus and future trends. Philos Trans R Soc Lond B Biol Sci 364: 2153-2166

Thompson RC (2015) Microplastics in the marine environment: Sources, consequences and solutions. In: Bergmann M, Gutow L, Klages M (eds) Marine Anthropogenic Litter. Springer, pp 185-200

Threlfall W (1968) The food of three species of gulls in Newfoundland. The Canadian Field Naturalist 82: $176-180$

Tokiwa Y, Calabia BP, Ugwu CU, Aiba S (2009) Biodegradability of plastics. International Journal of Molecular Sciences 10: 3722-3742

Torre M, Digka N, Anastasopoulou A, Tsangaris C, Mytilineou C (2016) Anthropogenic microfibres pollution in marine biota. A new and simple methodology to minimize airborne contamination. Marine Pollution Bulletin 113: 55-61

Trevail AM, Gabrielsen GW, Kühn S, Van Franeker JA (2015a) Elevated levels of ingested plastic in a high Arctic seabird, the northern fulmar (Fulmarus glacialis). Polar Biology 38: 975-981

Trevail AM, Kühn S, Gabrielsen GW (2015b) The State of Marine Microplastic Pollution in the Arctic. Norwegian Polar Institute, Kortrapport No 33, Tromso, Norway, pp 24

Turner A (2016) Heavy metals, metalloids and other hazardous elements in marine plastic litter. Marine Pollution Bulletin 111: 136-142

Turner A, Lau KS (2016) Elemental concentrations and bioaccessibilities in beached plastic foam litter, with particular reference to lead in polyurethane. Marine Pollution Bulletin 112: 265-270

\section{$\mathrm{U}$}

UNEP (2011) UNEP Year Book, 2011: Emerging issues in our global environment, United Nations Environmental programme, Nairobi, pp 79pp

Unger B, Bravo Rebolledo EL, Deaville R, Gröne A, IJsseldijk LL, Leopold MF, Siebert U, Spitz J, Wohlsein P, Herr H (2016) Large amounts of marine debris found in sperm whales stranded along the North Sea coast in early 2016. Marine Pollution Bulletin 112: 134-141 
V

Van Cauwenberghe L, Janssen CR (2014) Microplastics in bivalves cultured for human consumption. Environmental Pollution 193: 65-70

Van der Hal N, Yeruham E, Angel DL (2018) Dynamics in Microplastic Ingestion During the Past Six Decades in Herbivorous Fish on the Mediterranean Israeli Coast, Proceedings of the International Conference on Microplastic Pollution in the Mediterranean Sea, pp 159-165

Van der Veen I, de Boer J (2012) Phosphorus flame retardants: Properties, production, environmental occurrence, toxicity and analysis. Chemosphere 88: 1119-1153

Van Franeker J, Wattel J (1982) Geographical variation of the fulmar Fulmarus glacialis in the North Atlantic. Ardea 70: 31-44

Van Franeker J, SNS Fulmar Study Group (2013) Fulmar Litter EcoQO monitoring along Dutch and North Sea coasts-Update 2010 and 2011. IMARES, Texel, the Netherlands, pp 61

Van Franeker JA (1985) Plastic ingestion in the North Atlantic fulmar. Marine Pollution Bulletin 16: 367-369

Van Franeker JA, Bell PJ (1988) Plastic ingestion by petrels breeding in Antarctica. Marine Pollution Bulletin 19: 672-674

Van Franeker JA, Meijboom A (2002) Litter NSV - Marine litter monitoring by northern fulmars. A pilot study. Alterra, Alterra-Rapport 401, Wageningen, pp 72

Van Franeker JA (2004) Save the North Sea Fulmar Litter EcoQO manual Part 1: Collection and dissection procedures. Alterra, Wageningen, pp 38

Van Franeker JA (2005) Schoon Strand Texel 2005. Onderzoeksresultaten van de schoonmaakactie van het Texelse strand op 20 april 2005. Alterra Speciale Uitgave 2005/09 Texel, The Netherlands, pp 23

Van Franeker JA, Meijboom A (2006) Fulmar Litter EcoQO Monitoring in the Netherlands 1982-2004 in relation to EU Directive 2000/59/EC on Port Reception Facilities. Alterra, Report for the Ministry of Transport PwaWM, Texel, pp 41

Van Franeker JA, Flores H, Van Dorssen M (2009) The surface and under ice trawl (SUIT). Frozen Desert Alive-The role of sea ice for pelagic macrofauna and its predators PhD thesis University of Groningen: 181-188

Van Franeker JA, Blaize C, Danielsen J, Fairclough K, Gollan J, Guse N, Hansen PL, Heubeck M, Jensen JK, Le Guillou G, Olsen B, Olsen KO, Pedersen J, Stienen EW, Turner DM (2011) Monitoring plastic ingestion by the northern fulmar Fulmarus glacialis in the North Sea. Environmental Pollution 159: 2609-2615

Van Franeker JA, The SNS Fulmar Study Group (2011) Fulmar Litter EcoQO monitoring along Dutch and North Sea coasts in relation to EU Directive 2000-59/EC on Port Reception Facilities: results to 2009. IMARES, IMARES Report Nr C037/11, Texel

Van Franeker JA, Law KL (2015) Seabirds, gyres and global trends in plastic pollution. Environmental Pollution 203: 89-96

Van Franeker JA, Bravo Rebolledo EL, Hesse E, IJsseldijk LL, Kühn S, Leopold M, Mielke L (2018) Plastic ingestion by harbour porpoises Phocoena phocoena in the Netherlands: Establishing a standardised method. AMBIO: A Journal of the Human Environment 47: 387-397

Van Franeker JA, Kühn S (2019) Fulmar Litter EcoQO monitoring in the Netherlands - Update 2018. Wageningen Marine Research Report C077/19 \& RWS Centrale Informatievoorzieningen BM 19.16, pp 60

Van Sebille E, England MH, Froyland G (2012) Origin, dynamics and evolution of ocean garbage patches from observed surface drifters. Environmental Research Letters 7: 044040

van Sebille E, Wilcox C, Lebreton LCM, Maximenko N, Hardesty BD, van Franeker JA, Eriksen M, Siegel D, Galgani F, Law KL (2015) A global inventory of small floating plastic debris. Environmental Research Letters 10: 124006

Venables WN, B.D. R (2002) Modern Applied Statistics with S. Springer, New York, pp 503

Vendel AL, Bessa F, Alves VEN, Amorim ALA, Patrício J, Palma ART (2017) Widespread microplastic ingestion by fish assemblages in tropical estuaries subjected to anthropogenic pressures. Marine Pollution Bulletin 117: 448-455 
Verlis KM, Campbell ML, Wilson SP (2013) Ingestion of marine debris plastic by the wedge-tailed shearwater Ardenna pacifica in the Great Barrier Reef, Australia. Marine Pollution Bulletin 72: 244-249

Völker C, Kramm J, Wagner M (2019) On the Creation of Risk: Framing of Microplastics Risks in Science and Media. Global Challenges: 1900010

Votier SC, Grecian WJ, Patrick S, Newton J (2011) Inter-colony movements, at-sea behaviour and foraging in an immature seabird: results from GPS-PPT tracking, radio-tracking and stable isotope analysis. Marine Biology 158: 355-362

\section{W}

Walczowski W, Piechura J, Goszczko I, Wieczorek P (2012) Changes in Atlantic water properties: an important factor in the European Arctic marine climate. ICES Journal of Marine Science 69: 864-869

Walker WA, Coe JM (1990) Survey of marine debris ingestion by odontocete cetaceans. Shomura RS, Godfrey, M. L., Proceedings of the Second International Conference on marine debris, Honolulu, Hawaii, pp 747-774

Wang SW, Iverson SJ, Springer AM, Hatch SA (2007) Fatty acid signatures of stomach oil and adipose tissue of northern fulmars (Fulmarus glacialis) in Alaska: implications for diet analysis of Procellariiform birds. Journal of Comparative Physiology B 177: 893-903

Warham J (1996) The behaviour, population biology and physiology of the petrels. Academic Press

Waring G, Pace R, Quintal J, Fairfield C, Maze-Foley K (2004) US Atlantic and Gulf of Mexico marine mammal stock assessments-2003. NOAA Technical Memorandum NMFS-NE 182: 287

Wassmann P, Duarte CM, Agustí S, Sejr MK (2011) Footprints of climate change in the Arctic marine ecosystem. Global Change Biology 17: 1235-1249

Watts AJR, Porter A, Hembrow N, Sharpe J, Galloway TS, Lewis C (2017) Through the sands of time: Beach litter trends from nine cleaned north cornish beaches. Environmental Pollution 228: 416-424

Wei G-L, Li D-Q, Zhuo M-N, Liao Y-S, Xie Z-Y, Guo T-L, Li J-J, Zhang S-Y, Liang Z-Q (2015) Organophosphorus flame retardants and plasticizers: Sources, occurrence, toxicity and human exposure. Environmental Pollution 196: 29-46

Weimerskirch H, Chastel O, Cherel Y, Henden J-A, Tveraa T (2001) Nest attendance and foraging movements of northern fulmars rearing chicks at Bjørnøya Barents Sea. Polar Biology 24: 83-88

Welden NA, Cowie PR (2016) Environment and gut morphology influence microplastic retention in langoustine, Nephrops norvegicus. Environmental Pollution 214: 859-865

Welden NA, Abylkhani B, Howarth LM (2018) The effects of trophic transfer and environmental factors on microplastic uptake by plaice, Pleuronectes plastessa, and spider crab, Maja squinado. Environmental Pollution 239: 351-358

Werner S, Budziak A, van Franeker JA, Galgani F, Hanke G, Maes T, Matiddi M, Nilsson P, Oosterbaan L, Priestland E, Thompson R, Veiga J, Vlachogianni T (2016) Harm caused by Marine Litter. JRC Technical Report EUR 28317, MSFD GES TG Marine Litter - Thematic Report, pp 91

Wesch C, Elert AM, Wörner M, Braun U, Klein R, Paulus M (2017) Assuring quality in microplastic monitoring: About the value of clean-air devices as essentials for verified data. Scientific Reports 7: 5424

Weslawski JM, Ryg M, Smith TG, Oritsland NA (1994) Diet of ringed seals (Phoca hispida) in a fjord of West Svalbard. Arctic 47: 109-114

Wieczorek AM, Morrison L, Croot PL, Allcock AL, MacLoughlin E, Savard O, Brownlow H, Doyle TK (2018) Frequency of microplastics in mesopelagic fishes from the Northwest Atlantic. Frontiers in Marine Science 5: 1-9

Wilcox C, Van Sebille E, Hardesty BD (2015) Threat of plastic pollution to seabirds is global, pervasive, and increasing. Proceedings of the National Academy of Sciences 112: 11899-11904 
Wilcox C, Puckridge M, Schuyler QA, Townsend K, Hardesty BD (2018) A quantitative analysis linking sea turtle mortality and plastic debris ingestion. Scientific Reports 8: 12536

Woodall LC, Sanchez-Vidal A, Canals M, Paterson GLJ, Coppock R, Sleight V, Calafat A, Rogers AD, Narayanaswamy BE, Thompson RC (2014) The deep sea is a major sink for microplastic debris. Royal Society Open Science 1: 140317

WoRMS (2019) World Register of Marine Species. Available from http://www.marinespecies.org. Accessed 2019-06-30.

WRAP (2014) UK voluntary carrier bag monitoring - 2013 data. In: Yamashita R, Takada H, Fukuwaka MA

Watanuki Y (2011) Physical and chemical effects of ingested plastic debris on short-tailed shearwaters, Puffinus tenuirostris, in the North Pacific Ocean. Marine Pollution Bulletin 62: 2845-2849

$\mathbf{Y}$

Youngren SM, Rapp DC, Hyrenbach KD (2018) Plastic ingestion by Tristram's Storm-petrel (Oceanodroma tristrami) chicks from French frigate shoals, Northwestern Hawaiian Islands. Marine Pollution Bulletin 128: 369-378

\section{$\mathrm{Z}$}

Zarfl C, Matthies M (2010) Are marine plastic particles transport vectors for organic pollutants to the Arctic? Marine Pollution Bulletin 60: 1810-1814 
Addenda 


\section{Summary}

Plastic litter in the marine environment has been observed to be omnipresent on a global scale. Northern fulmars (Fulmarus glacialis) ingest plastics regularly. For this doctoral thesis different pathways of plastics in northern fulmars were investigated. These pathways include the direct ingestion of plastic by fulmars, the uptake of plastics through their potentially contaminated prey and the transfer of plastic-related chemicals into the fulmars digestive system. Additionally, new methods were developed or validated to study plastics ingested by marine organisms thoroughly. Finally, a global overview of plastic ingestion and entanglement by marine species is presented.

\section{Direct ingestion of plastic by fulmars}

We studied the uptake of plastics by fulmars from different regions of the North Atlantic. Data on plastic ingestion was available for the North Sea, the UK, mainland Norway and Arctic Canada. For this thesis, baseline data for Iceland and Svalbard were established. In Iceland (Chapter 2), 79\% of the fulmars ingested plastics; on average these birds contained 6 pieces, weighing on average 0.13 gram per bird. In Svalbard (Chapter 3) plastic ingestion was slightly higher; $87.5 \%$ of the fulmars had plastic in their stomach, on average 13 pieces, weighing 0.08 gram. Fulmars studied in remote areas in Iceland or Svalbard have less plastic in their stomachs than fulmars from the highly industrialized North Sea; thus, plastic ingestion in fulmars reflects the local abundance of plastics in the environment. Despite the remoteness of Svalbard, the higher plastic intake in fulmars may be explained by ocean currents that transport plastics from more polluted areas to the Arctic Ocean.

\section{Plastic uptake through trophic transfer}

The uptake of plastic through prey was studied to quantify the proportion of plastic that marine top predators may ingest. Polar cod (Boreogadus saida) is a common prey fish for Arctic seabirds and spends part of its life cycle underneath the Arctic sea ice. Sea ice is thought to be a sink for plastics that are brought to the Arctic with currents from more polluted southern areas. Out of 72 studied individuals, two polar cods were found with one small plastic particle each in their stomachs, one fish from underneath the sea ice and the other one from open water close to the coast of Svalbard (Chapter 4).

To dissolve organic matter in fish stomachs and to facilitate the search for small microplastics, the use of potassium hydroxide $(\mathrm{KOH})$ was proposed. Our studies confirm that $\mathrm{KOH}$ dissolves organic matter, without impacting the integrity of most plastics (Chapter 5). Therefore $\mathrm{KOH}$ was used to analyse a large amount of fish from the North Sea. In combination with earlier datasets, more than 4000 fish from the North Sea area were analysed (Chapter 6). Similar to polar cod, overall frequency of occurrence was low for the majority of the 15 species (1.8\%). The exception was Atlantic cod (Gadus morhua), 
where $12.3 \%$ of the individuals contained plastic. These results show that the sampling scheme should be adapted and standardized when studying plastic uptake. This should be considered when proposing fish species for long-term monitoring of plastic ingestion.

In both datasets, polar cod and the assembly of North Sea fish (Chapter $4 \& 6$ ), textile fibres were encountered in the samples. These may either be ingested by the organism but can also originate from secondary atmospheric contamination, when the sample is exposed to the air. The distinction of the origin of these fibres is extremely difficult. Controls running parallel to the sample analysis indicate a clear positive relationship between the duration of exposure and the number of fibres encountered. Therefore, for polar cod and North Sea fish, most fibres were determined to be airborne contamination and fibres were excluded from detailed spatial and temporal analysis of plastics.

Low amounts of plastic encountered in fish from the Arctic Ocean and the North Sea indicate that trophic transfer of plastics from fish to fulmars and other predators may be negligible. Additionally, the small sized plastics encountered in fish may pass through the gastrointestinal tract of fulmars without accumulation.

\section{Plastic characteristics and transfer of plastic-associated chemicals}

In Chapter 7, spatial and temporal variations of polymer types ingested by fulmars were studied and compared to related seabird species from the Southern Ocean. In preparation for this study, two different polymer analysis techniques have been compared: Near Infrared Spectroscopy (NIR) and Fourier Transform Infrared Spectroscopy (FTIR). Both methods are suitable to identify plastics from seabird stomachs. However, NIR performed less reliably on foams and dark particles, while FTIR in some cases confused natural hard prey items (e.g. lish eye lenses or squid beaks) with artificial polymers (Chapter 7). Polymer composition ingested by northern fulmars has changed throughout three decades. Furthermore, composition differs between regions of the North Atlantic and on a global scale respectively. These changes are closely related to changes in plastic categories, such as industrial pellets, sheets, threads, foam and fragments. The uptake of different plastic categories and related polymer types is expected to reflect plastic production and disposal fluxes on a global scale.

A microplastic mixture was collected, processed and analysed for future assessment studies of potential impacts on organisms caused by marine plastic debris (Chapter 8). Macroplastic litter was collected from a Dutch beach. Collected plastic items were cryomilled to microplastic size $(<5 \mathrm{~mm})$. Physical and chemical analyses were performed to gather information on particle size, number and shape, polymer composition, metal concentrations and integrated additives. The particles of microplastic mixture were irregular in size and shape. The majority of plastic items was made of polyethylene and polypropylene and a diverse range of (heavy) metals and additives, such as plasticizers, UV blockers, and flame retardants were found. This mixture can improve the quality of relevant experiments under realistic conditions, as previous studies usually rely on homogenous and pristine plastic particles. 
Our microplastic mixture was used to study the transfer of potentially toxic plastic additives to the stomach oil of northern fulmars (Chapter 9). Stomach oil is a substance produced by procellariform seabirds from dietary remains, used as energy storage. For different periods of time, ranging from eight hours up to three months, stomach oil was exposed to the plastic mixture and to a mixture of polystyrene foam. From both plastic mixtures, some of the identified additives were leaching to the stomach oil, such as precursors to resins and copolymers, plasticizers, flame retardants, UV stabilizers, antioxidants and preservatives. Time and duration of leaching varied by substance (Chapter 9).

\section{Global overview of plastic ingestion by marine wildlife}

Species of all sizes, habitats and foraging strategies have been found to ingest plastics. However, the rate of ingestion can vary considerably between species and species groups. In total, 915 marine species have been found either to ingest or to get entangled in marine plastic litter (Chapter 10). Plastic ingestion by marine birds, mammals and turtles was quantified based on a literature review. However, quantification was hampered by inconsistent methods used in these studies. On average, one third of all seabirds and turtles that have been studied for plastic ingestion were found to contain plastic in their stomachs (27.7 and 32\%, respectively). Within all marine mammals studied, $4.4 \%$ were found with ingested plastics. Especially in seabirds, plastic uptake differs between species groups. The species group with the highest frequency of occurrence, and the highest numbers of plastic particles ingested, is the order of Procellariiformes (albatrosses, petrels, shearwaters, fulmars, storm- and diving-petrels) with $41.5 \%$.

\section{Conclusions}

The results of this thesis show that the northern fulmar is a useful species for studying plastic ingestion by marine wildlife. To inform governments and society, standardized method protocols should be established to allow large-scale comparisons of uptake and effect of plastics in marine wildlife. Even in remote (sub)arctic regions the level of ingestion is high. The fulmar belongs to the seabird order most affected by plastic ingestion. Direct ingestion of plastic is the main driver of uptake; secondary ingestion of plastics via their prey only plays a minor part. Due to the constant uptake and the transfer of potentially toxic chemicals, fulmars are particularly prone to negative effects that may occur when ingesting plastics. 


\section{Samenvatting}

Plastic zwerfvuil is in alle oceanen een algemeen verschijnsel geworden. Noordse stormvogels (Fulmarus glacialis) slikken plastic regelmatig in. Voor dit proefschrift zijn verschillende manieren van opname van plastic door stormvogels onderzocht. Er is een directe opname van plastic door stormvogels maar ook een indirecte opname, bijvoorbeeld wanneer een prooi zelf plastic heeft gegeten. Tijdens de spijsvertering kunnen mogelijk stoffen uit het plastic naar de stormvogel overgedragen worden. Er zijn nieuwe methoden om opname van plastic goed te kunnen bestuderen ontwikkeld en getest. Tenslotte is in een globaal overzicht van zeedieren aangegeven, welke plastic ingeslikt hebben of erin verstrikt zijn geraakt.

\section{Directe opname van plastic zwerfvuil door stormvogels}

De opname van plastics door stormvogels is voor verschillende regio's van de noordelijke Atlantische Oceaan bestudeerd. Er waren al gegevens bekend van de Noordzee, het Verenigd Koninkrijk, Noorwegen en het Arctische gebied van Canada. Voor dit proefschrift zijn er gegevens voor IJsland en Svalbard toegevoegd. Op IJsland (Hoofdstuk 2), hebben $79 \%$ van de onderzochte stormvogels plastic in hun maag, gemiddeld 6 stukjes, die per vogel 0.13 gram wegen. Op Svalbard (Hoofdstuk 3) was er meer plastic gevonden, 87.5\% van de vogels had plastic ingeslikt, gemiddeld 13 stukken en per vogel 0.08 gram. Stormvogels uit afgelegen gebieden zoals IJsland en Svalbard hebben minder veel plastic in hun maag dan stormvogels uit het drukke Noordzeegebied. De aanwezigheid van plastic in stormvogels weerspiegelt dus de aanwezigheid van plastic in het mariene milieu. Ondanks de afgelegenheid van de Svalbard Archipel spoelt de zeestroming veel vervuiling van zuidelijke gebieden naar het noorden. De wat grotere hoeveelheid plastic in stormvogels zou daardoor verklaard kunnen worden.

\section{Plastic opname door prooivis}

De opname van plastic door vis is onderzocht om een mogelijke bijdrage van dit plastic aan de aantallen in stormvogels gevonden plastic te kunnen inschatten. Poolkabeljauw (Boreogadus saida) is een algemene prooivis voor Arctische zeevogels en brengt een deel van zijn leven dicht onder het zee-ijs door. Van zee-ijs is bekend, dat het grote hoeveelheden plastic op kan slaan, dat door zeestromingen uit het meer vervuilde zuiden het Arctische gebied kan bereiken. Van 72 onderzochte vissen hadden er 2 vissen een stuk plastic in hun maag. Één vis werd direct onder het ijs opgevist, de andere uit open water langs de kust.

Om organisch materiaal in een monster op te lossen en op die manier het zoeken naar klein plastic in magen te vergemakkelijken, werd het gebruik van kalium hydroxide $(\mathrm{KOH})$ voorgesteld. Onze studie bevestigt dat $\mathrm{KOH}$ een geschikt middel is om organisch materiaal 
op te lossen, zonder het aanwezige plastic aan te tasten (Hoofdstuk 5). Daarom werd $\mathrm{KOH}$ ook gebruikt in een studie, waarbij grote hoeveelheden vis uit de Noordzee werden onderzocht. Samen met eerdere databestanden konden meer dan 4000 vismagen geanalyseerd worden (Hoofdstuk 6). In vergelijking met poolkabeljauw zijn de aantallen gevonden plastics in de 15 soorten Noordzeevis laag (1.8\%). Een uitzondering was de kabeljauw (Gadus morhua), waar $12.2 \%$ van alle onderzochte vissen met plastic in hun maag gevonden werden. De resultaten laten zien dat gestandaardiseerd werk nodig is om plastic in vis te bestuderen en te kunnen vergelijken. Dit is vooral belangrijk wanneer vissoorten voor lange-termijnonderzoek gebruikt gaan worden.

In beide monsters van poolkabeljauw en Noordzeevis (Hoofdstuk $4 \& 6$ ) werden regelmatig hele kleine en lichte kledingvezels aangetroffen. Deze kunnen door de vis zelf ingeslikt zijn maar ook als secundaire vervuiling tijdens de analyse in het monster terecht gekomen zijn als deze aan lucht blootgesteld is. Het is erg ingewikkeld om het verschil tussen die twee types vezel te herkennen. Kontrolemonsters, die parallel met de echte monsters lopen, laten een duidelijk verband zien met de tijd van blootstelling en het aantal vezels. Daarom werden de meeste gevonden vezels als luchtcontaminatie beschouwd en uitgesloten uit verdere analyse.

Lage aantallen plastic in vis uit het Arctische gebied en de Noordzee laten zien, dat de overdracht van plastic van vis naar stormvogel onwaarschijnlijk is. Het kleine formaat van het in vis gevonden plastic, draagt niet aanzienlijk bij aan de hoeveelheid plastic in stormvogelmagen en kan mogelijk makkelijk weer uitgescheiden worden.

\section{Plastic eigenschappen en overdracht van chemicaliën}

In Hoofdstuk 7 worden door stormvogels ingeslikte plastics geïdentificeerd. Dit gebeurde op verschillende plaatsen in de noordoostelijke Atlantische Oceaan en ook door drie decennia heen. De resultaten werden vervolgens vergeleken met plastic uit nauw verwante vogelsoorten die rond Antarctica voorkomen. Ter voorbereiding zijn twee verschillende analyse technieken met elkaar vergeleken, Near Infrared Spectroscopy (NIR) en Fourier Transform Infrared Spectroscopy (FTIR). Beide methodes blijken geschikt voor de analyse van plastics uit vogelmagen. NIR was iets minder betrouwbaar in de analyse van piepschuim en donkere plastics, terwijl FTIR vaak natuurlijke prooiresten (zoals ooglenzen van vis en inktvis, visbotten en veren) niet van plastic kan onderscheiden. De samenstelling van verschillende soorten plastic in stormvogelmagen verandert door de decennia heen. Ook de samenstelling van plastic in verschillende regio's binnen de NoordAtlantische Oceaan en de Zuidelijke Oceaan varieert. De veranderingen hangen samen met de verschillende plastic-categorieën, zoals industriële pellets, vellen, draden, piepschuim en plasticscherven. De opname van verschillende plastictypes weerspiegelt waarschijnlijk de globale productie van plastic en verspreiding ervan in het milieu.

Een mengsel van plastics, representatief voor wat in de Noordzee drijft, werd verzameld en klein gemalen $(<5 \mathrm{~mm})$. Dit mengsel kan gebruikt worden in toekomstige studies aan mogelijke schade van plastic op dieren (Hoofdstuk 8). Fysieke en chemische analyses 
zijn uitgevoerd om informatie te verkrijgen over de deeltjesgrootte en vorm, de samenstelling, de concentratie van metalen en toegevoegde stoffen. De deeltjes in dit mengsel waren onregelmatig in grootte en vorm. De meeste stukken waren gemaakt van polyethyleen (PE) en polypropyleen (PP) en bevatten een grote diversiteit aan metalen en toegevoegde stoffen zoals weekmakers, brandvertragers en UV-blokkers. Met dit mengsel kunnen realistischere experimenten uitgevoerd worden dan met de gebruikelijke verse plastic materialen die vaak homogeen in grootte, vorm en chemische samenstelling zijn.

Hetzelfde mengsel werd gebruikt om de overdracht van mogelijk giftige stoffen in het plastic naar maagolie van stormvogel te bestuderen (Hoofdstuk 9). Maagolie wordt door stormvogelachtigen geproduceerd uit hun natuurlijke voedsel en wordt gebruikt als energieopslag. De blootstelling van maagolie aan dit mengsel en ook een mengsel van alleen maar piepschuim duurde tussen 8 uur en 90 dagen. Van beide plasticmengsels lekten er verschillende toegevoegde stoffen naar de maagolie, zoals precursors van kunststofharsen, antioxidanten, weekmakers, brandvertragers, UV stabilisatoren, en conserveermiddelen. De tijd en duur van de lekkage varieert per stof.

\section{Wereldwijd overzicht van plastic opname}

Dieren van allerlei soorten en maten zijn gevonden met plastic in hun magag. In totaal zijn 915 soorten gevonden, die of plastic in hun maag hebben, of in plastic verstrikt zijn geraakt (Hoofdstuk 10). In een literatuuroverzicht werd de opname van plastic in zeevogels, zeezoogdieren en zeeschildpadden gekwantificeerd. De analyse van deze data werd belemmerd door inconsistente methodes die in de onderliggende studies gebruikt worden. De hoeveelheid plastic varieert in verschillende soorten. Gemiddeld hebben een derde van alle onderzochte zeevogels en zeeschildpadden plastic in hun maag (27.7 en 32\%). Bij alle onderzochte zeezoogdieren werd in $4.4 \%$ plastic aangetroffen. Vooral in zeevogels is de variatie van plasticopname groot tussen de verschillende soorten. De groep met de hoogste aantallen vogels binnen één soort en de hoogste aantallen plastics per vogel zijn de Procellariiformes (buissnavelachtigen zoals albatrossen en (pijl)stormvogels) waarvan 41.5\% van alle onderzochte dieren plastic bevatten.

\section{Conclusie}

De resultaten van dit proefschrift tonen aan, dat de noordse stormvogel een geschikte soort is om opname van plastic in dieren te bestuderen. Om beleidmakers goed te kunnen informeren en adviseren zijn meer gestandaardiseerde protocollen nodig. Zelfs in afgelegen (sub)Arctische gebieden zijn de hoeveelheden van ingeslikt plastic hoog. Stormvogels behoren tot de familie van vogels, die het meest frequent plastic inslikken. Directe opname speelt hierbij een duidelijk belangrijkere rol dan de opname via prooivis. Door de regelmatige opname van plastic en de aangetoonde overdracht van mogelijk giftige stoffen zijn stormvogels zeer gevoelig voor mogelijke negatieve effecten die het eten van plastic veroorzaakt. 


\section{Zusammenfassung}

Plastikmüll in den Meeren ist eine weit verbreitete Art der Umweltverschmutzung und kann in allen Ozeanen gefunden werden. Eissturmvögel (Fulmarus glacialis) fressen regelmäßig Plastik. Für diese Doktorarbeit wurden verschiedene Wege der Aufnahme von Plastik erforscht. Die verschiedenen Wege beinhalten die direkte Aufnahme von Plastik durch Eissturmvögel, die indirekte Aufnahme (z. B. durch Fisch mit Plastik im Magen) und die Übertragung von plastikspezifischen Chemikalien in das Verdauungssystem des Eissturmvogels. Dabei wurden neue Methoden entwickelt und bestehende Methoden getestet. Zusätzlich wurde eine globale Übersicht von allen Arten im Meer erarbeitet, die entweder Plastik im Magen hatten, oder aber darin verstrickt waren.

\section{Direkte Aufnahme von Plastik durch Eissturmvögel}

Daten für die Plastikaufnahme in Eissturmvögeln wurden für den Nordatlantik gesammelt. Für die Nordsee, Großbritannien, Norwegen und Arktisch Kanada waren bereits Daten erhoben. Im Rahmen dieser Doktorarbeit konnten neue Daten für Island und Svalbard (Spitzbergen) erworben werden. Auf Island (Kapitel 2), wurde in $79 \%$ der untersuchten Eissturmvögel Plastik festgestellt. Im Durchschnitt hatten die Vögel 6 Stück Plastik im Magen, die per Vogel 0,13 Gramm wogen. Auf Svalbard war diese Rate leicht erhöht, 87,5 $\%$ aller Eissturmvögel hatten Plastik im Magen, durchschnittlich 13 Stück, die per Vogel 0,08 Gramm wogen (Kapitel 3). Eissturmvögel in abgelegenen Orten, so wie Island, haben weniger Plastik in ihren Mägen als Vögel aus der hochindustrialisierten Nordsee, das bedeutet, dass Eissturmvögel die lokale Menge des Plastiks im Meer widerspiegeln. Die leicht erhöhten Werte auf dem abgelegenen Svalbard-Archipel lassen sich vermutlich durch Meeresströmungen erklären, die verschmutztes Wasser aus dem Süden bis in die Arktis transportieren.

\section{Aufnahme von Plastik durch Beutetiere}

Die Aufnahme von Plastik in Beutefisch wurde untersucht, um die relative Menge des so erworbenen Plastiks in Eissturmvögeln festzustellen. Polardorsch (Boreogadus saida) ist ein typischer Beutefisch für arktische Seevögel und verbringt Teile seines Lebens unter dem Meereis. Meereis wird verdächtigt, große Mengen des Plastiks, welches über Meeresströmungen in die Arktis transportiert wird, aufzufangen und einzulagern. Von 72 untersuchten Fischen, hatten 2 Polardorsche jeweils ein Stück Plastik in ihrem Magen. Einer der Fische wurde direkt unter dem Meereis gefangen, der andere im offenen Wasser nahe der Küste Svalbards (Kapitel 4).

Um organisches Material aufzulösen und so die Suche nach kleinen Plastikstücken zu vereinfachen, wurde der Gebrauch von Kaliumhydroxid (KOH) vorgeschlagen. Unsere Studien bestätigen, dass KOH organisches Material auflöst, ohne Plastik zu beschädigen 
(Kapitel 5). Darum wurde KOH auch genutzt, um große Mengen Nordseefisch auf Plastik zu untersuchen. Zusammen mit früheren Datenbeständen konnten so mehr als 4000 Fischmägen aus der Nordsee analysiert werden (Kapitel 6). Ähnlich wie beim Polardorsch wurde nur wenig Plastik in den 15 untersuchten Arten gefunden (1,8\%). Eine Ausnahme bildete der Atlantische Dorsch (Gadus morhua), hier wurde in 12,3 \% aller Fälle Plastik dokumentiert. Die Ergebnisse zeigen, dass standardisierte Protokolle nötig sind, wenn Plastik in Tierarten untersucht wird. Mit geeigneten Protokollen könnte dann auch die Politik gut beraten werden, wenn Fischarten für Langzeituntersuchungen erörtert werden.

In beiden Proben, Polardorsch und Nordseefisch (Kapitel 4 \& 6), wurden kleine, sehr leichte Textilfasern gefunden. Diese können entweder durch die Fische selbst aufgenommen werden, oder aber aufgrund ihres geringen Gewichtes während der Probenanalyse aus der Luft in die Probe gelangt sein. Die Unterscheidung dieser zwei Quellen ist extrem schwer. Kontrollproben, die parallel zu den echten Proben genommen wurden, zeigen einen deutlichen Zusammenhang zwischen der Dauer, die die Probe an der offenen Luft war und der Anzahl der gefunden Fasern. Aus diesem Grund wurden die meisten Fasern in Polardorsch und Nordseefisch als sekundäre Verschmutzung eingeschätzt und aus allen weiteren Datenanalysen entfernt.

Die niedrige Anzahl von Plastik in Polardorsch und Nordseefisch deutet darauf hin, dass die Übertragung von Plastik durch Fische auf Eissturmvogel und andere Fressfeinde nur geringfügig ist. Die oft sehr kleinen im Fisch gefundenen Plastikteile werden von Eissturmvögeln vermutlich schnell wieder ausgeschieden.

\section{Eigenschaften von Plastik und die Übertragung von plastikspezifischen Chemikalien}

In Kapitel 7 wurde die räumliche und zeitliche Verbreitung von durch Eissturmvögel verschluckte Kunststoffarten analysiert und verglichen mit Plastik aus nah verwandten Vogelarten aus dem südlichen Polarmeer. In der Vorbereitung zu dieser Studie wurden zwei verschiedene Analysemethoden verglichen: Nahinfrarotspektroskopie (NIR) und Fourier-Transformations-Infrarotspektroskopie (FTIR). Beide Methoden scheinen geeignet, Plastik aus Vogelmägen zu identifizieren. Allerdings zeigt die NIR Schwächen bei der Identifikation von dunklen Plastikpartikeln und Schaumstoffen, während FTIR manchmal natürliche Beutereste, wie zum Beispiel Fischaugen oder -knochen und Tintenfischkiefer, mit Plastik verwechselt. Die Zusammenstellung der Kunststoffarten in Eissturmvögeln hat sich durch die letzten drei Jahrzehnte verändert. Unterschiede wurden auch bei der Zusammenstellung in verschiedenen Regionen, einschließlich dem Südpolarmeer, festgestellt. Die Veränderungen sind eng verknüpft mit verschiedenen Plastikkategorien, wie zum Beispiel industrielles Granulat, Schaumstoff, Folien und Fragmente. Die Aufnahme von verschiedenen Plastikkategorien und dementsprechenden Kunststoffarten durch Sturmvögel reflektiert die globale Plastikproduktion und weltweite Abfallströme.

Eine Plastikmischung wurde zusammengestellt und analysiert, um zukünftige Experimente über mögliche Effekte von Plastikaufnahmen in Tieren möglich zu machen (Kapitel 
8). Makroplastik wurde an einem holländischen Strand gesammelt. Das gesammelte Plastik wurde kryogen zu Mikroplastik $(<5 \mathrm{~mm}$ ) gemahlen. Physikalische und chemische Analysen wurden ausgeführt, um mehr Information über Partikelgröße und -form, Kunststoffzusammenstellung, Metallkonzentrationen und integrierte Zusatzstoffe im Plastik zu erhalten. Die Partikel in der Mischung waren unterschiedlich groß und hatten unterschiedliche Formen. Ein Großteil der Mischung bestand aus Polyethylen und Polypropylen und eine große Vielfalt von (Schwer-)Metallen und Zusatzstoffen wurde gefunden, wie Weichmacher, UV-Filter und Flammschutzmittel.

Die durch uns entwickelte Plastikmischung wurde eingesetzt, um die mögliche Übertragung von möglicherweise giftigen Zusatzstoffen aus Plastik auf Magenöl von Eissturmvögeln zu studieren (Kapitel 9). Magenöl ist eine Substanz, die von sturmvogelähnlichen Arten (Röhrennasen; Procellariiformes) aus erbeuteter Nahrung produziert und als direkter Energiespeicher genutzt wird. Für unterschiedliche Zeiträume, zwischen 8 Stunden und 90 Tagen, wurde das Öl mit der Plastikmischung und separat auch mit Styroporflocken vom Strand vermischt. Von beiden Mischungen übertrugen sich einige Zusatzstoffe, wie Vorformen von Epoxidharzen, Weichmacher, Antioxidationsmittel, Flammschutzmittel, UV-Stabilisatoren und Konservierungsstoffe in das Magenöl. Zeitpunkt und Dauer dieses Übertragungsprozesses variierten per Substanz.

\section{Weltweite Übersicht von Plastikaufnahme in Meerestieren}

Tierarten aller Größen, Habitate und Nahrungsstrategien wurden bereits mit Plastik in ihrem Magen gefunden. Die Aufnahmeraten jedoch unterscheiden sich stark in verschiedenen Arten oder Artgruppen. Insgesamt wurden 915 Arten beschrieben, die entweder Plastik verschluckt oder sich im Plastik verstrickt hatten (Kapitel 10). Die Plastikaufnahme in Seevögeln, Meeressäugern und Meeresschildkröten wurde, basierend auf einer Literaturanalyse, quantifiziert. Allerdings wurde diese Analyse erschwert durch den Gebrauch von inkonsistenten Methoden in den einzelnen Studien. Im Durchschnitt haben etwa ein Drittel aller auf Plastik hin untersuchten Seevögel und Schildkröten Plastik in ihrem Magen (jeweils 27,7 und 32 \%). Von allen untersuchten Meeressäugern hatten 4,4 \% Plastik verschluckt. Vor allem in Seevögeln variierte der Durchschnitt zwischen verschiedenen Artgruppen. Die Artgruppe mit der höchsten Auftrittshäufigkeit von 41,5 \% sind die Röhrennasen, zu denen neben dem Eissturmvogel auch Albatrosse, Sturmschwalben und andere Sturmvögel gehören.

\section{Fazit}

Die Ergebnisse dieser Doktorarbeit zeigen, dass der Eissturmvogel eine geeignete Seevogelart ist, um die Plastikaufnahme in Meerestieren zu studieren. Um Politik und Gesellschaft gut informieren zu können, sind standarisierte Methoden notwendig, die großangelegte Studien vereinfachen können. In diesen Studien können dann mögliche negative Effekte der Plastikaufnahme auf Tiere untersucht und besser eingeschätzt 
werden. Selbst in abgelegenen (Sub-)Arktischen Gebieten ist das Level der Plastikaufnahmen hoch. Der Eissturmvogel ist eine der Arten, die am meisten betroffen sind von Plastik in ihren Mägen. Die direkte Aufnahme scheint der relevanteste Weg zu sein, die indirekte Aufnahme via Beutefisch spielt nur eine untergeordnete Rolle. Durch die konstante Aufnahme und die Übertragung von plastikspezifischen, möglicherweise giftigen Substanzen, sind Eissturmvögel besonders anfällig für negative Effekte, die auftreten können durch das Fressen von Plastikteilen. 


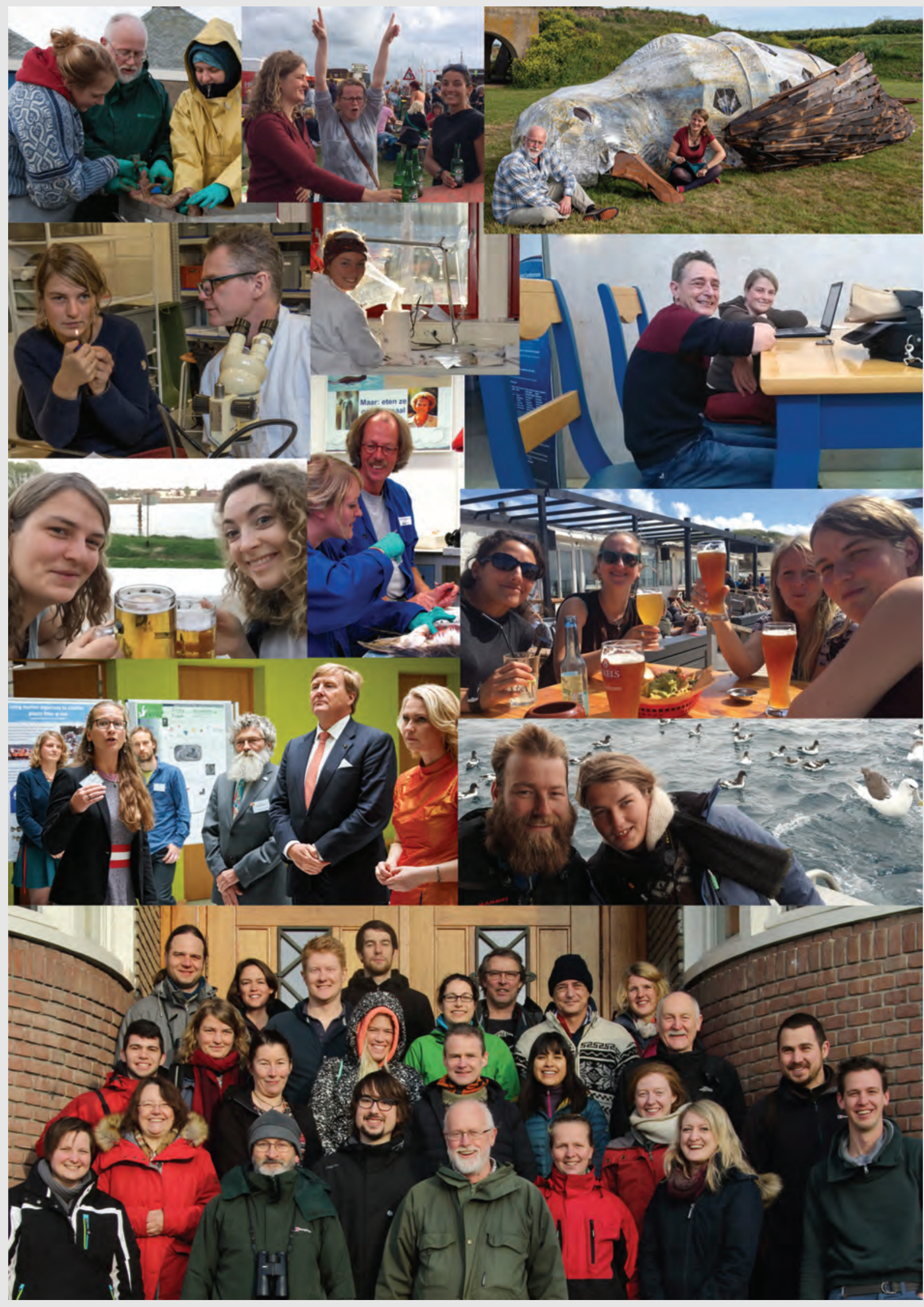




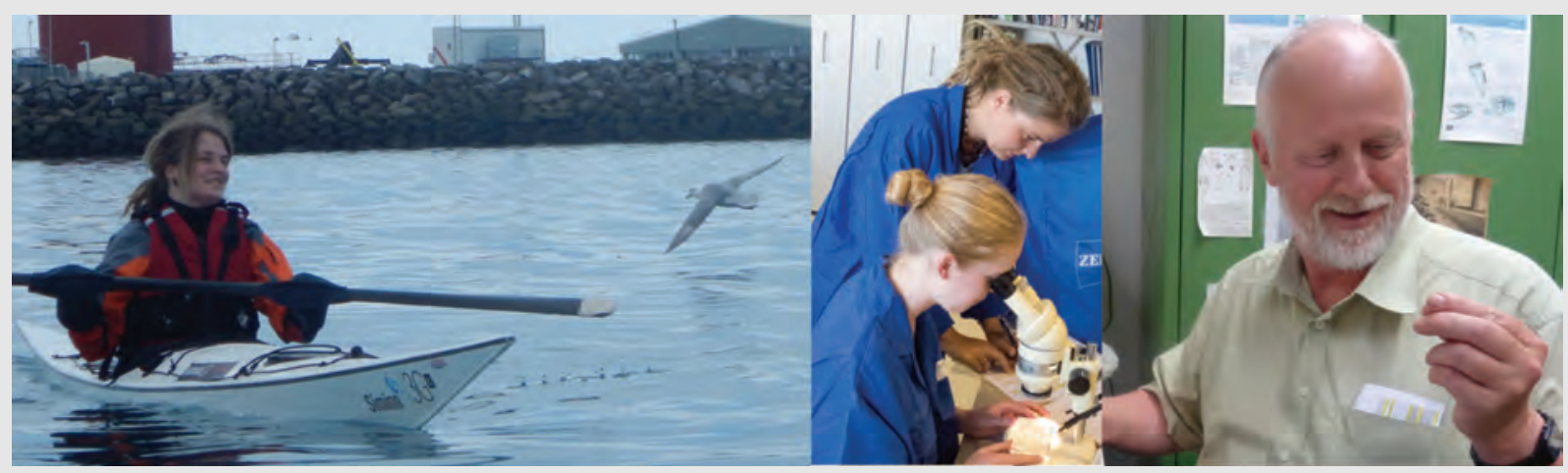

\section{Acknowledgements}

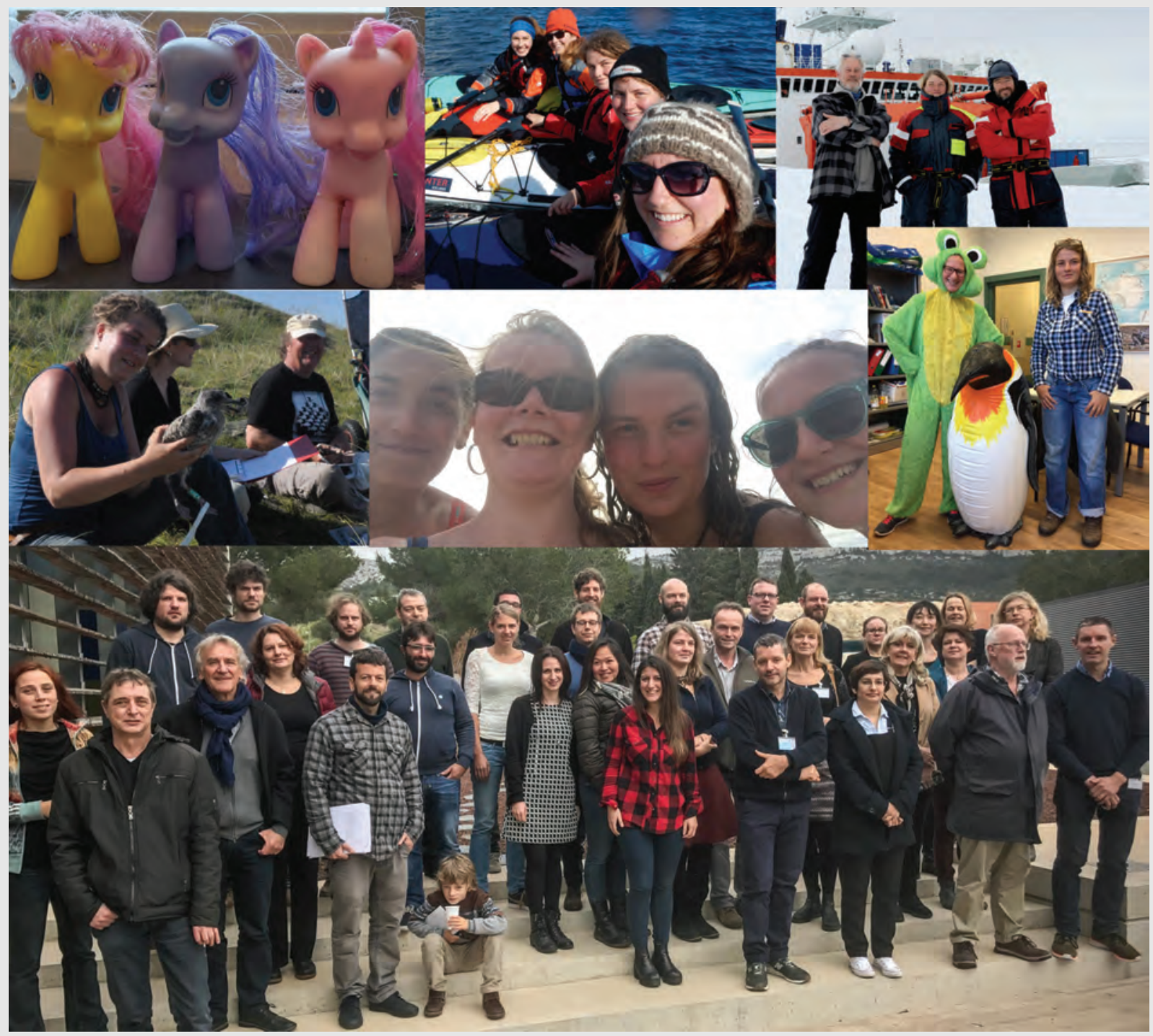


Jan, waar moet ik beginnen? Alle kansen die jij mij in de afgelopen 10 jaar hebt gegeven zijn ontelbaar. Het begon met een twijfel, je twijfelde, of ik erin zou slagen om IJslandse stormvogels voor plasticonderzoek te verzamelen. Op IJsland draaide ik ieder steen om, om erachter te komen, dat de oplossing maar een paar deuren verderop zat. Met de magen van 40 vogels in de bagage kwam ik terug naar Texel en mocht ik de maaginhoud onder jouw begeleiding analyseren. Daarmee niet genoeg, ik mocht ook een eerste publicatie schrijven, met als resultaat een hoofdstuk in deze proefschrift. Vanaf dat moment werken we samen aan verschillende projecten, ontstond er een Master's thesis en in December 2016 kregen we eindelijk de verlossende email: ik mocht in dienst als aio. Jouw enthousiasme en passie voor zeevogels, je ontzettend kritische blik op de (onderzoeks)wereld, en vooral jouw (bijna) onuitputtelijke geduld maken je een fantastische begeleider en mentor, waar ik zo veel van heb mogen leren. Dank voor alle discussies, alle uitleg en advies, je geduld, gezellige borrels en maaltijden, voor het openschuiven van deuren met geweldige mogelijkheden erachter, zowel in plastic- als ook in poolonderzoek. Han and Geir, you decided to accompany me as Promotors during my PhD, I want to thank you for your supervision. You've trusted in Jan and his abilities to supervise me. Therefore I had all the freedom to develop myself towards a scientist and I am grateful for that!

Sinds mijn eerste dag bij IMARES heb jij, André, voor me klaar gestaan! Van jou heb ik geleerd hoe je plastic uit magen sorteert en onderscheidt van natuurlijk voedsel. Voor je geduld, vriendelijkheid en enthousiasme zal ik je altijd dankbaar zijn. Ik denk dat er weinig mensen zijn die IMARES zo goed kennen, jij weet alles! te vinden, van gereedschap tot foto's uit de oude doos. Weet jij een keer iets niet, zoek je het zeer grondig uit, dat heb ik altijd bewonderd! Je was er op momenten dat ik je echt nodig had (oefenen met auto rijden naar de Eems, na lange diensten in de vogelkist op de Polarstern, of als er eens een autospiegel stuk gaat, om maar een paar enkele momenten te noemen). Samen met jou is het altijd gezellig: goede muziek, gezellige borrels met feestverlichting en niet te vergeten iedere ochtend een duimbreed koffie... Ik ben je ontzettend dankbaar voor onze vriendschap!

Vier jaar lang aan mijn proefschrift werken was onmogelijk geweest zonder mijn twee lievelings-mede-aio's Marin en Fokje! Onze vriendschap heeft op zo veel vlaktes geholpen! Eeuwig dank voor jullie allebei, om mij met zoveel geduld te helpen met alle statistiek en $\mathrm{R}$ drama's van de afgelopen jaren! Jullie hebben vele versies van manuscripten van mij gelezen, jullie kritische commentaar heeft geholpen om deze beter en gestructureerder te maken, dank daarvoor! Ik heb ontzettend genoten van alle activiteiten die wij samen als inofficiële 'feestcommissie' georganiseerd hebben. Zonder alle kleine en grote koffiepauzes op de trap of op het dak van de ZVS was de tijd als aio heel veel moeilijker verlopen! Jullie vrolijkheid en goede humor hebben de tijd als aio bij WMR echt heel erg leuk en gezellig gemaakt!

Lieve studenten, Bernike, Anastasia, Ailynn en Marrit, jullie hebben allemaal ongelofelijk veel tijd achter de microscoop doorgebracht om mij te helpen, plastic in vis te vinden. Jullie geduld en volhoudingsvermogen verdienen allergrootste respect. Plastic in onze vis was 
zelden en vaak piepklein, nonnenwerk om het zo maar te noemen! Door jullie te mogen begeleiden heb ik veel kunnen leren, ook daarvoor dank!

Ik mag mij gelukkig noemen met veel leuke collega's die bij IMARES/WMR werken en werkten. Het zijn er erg veel, dus daarom dat ik niet alle op kan noemen, maar ik wil jullie wel allemaal ontzettend bedanken voor de gezelligheid en het altijd klaar staan voor vragen, uitleg, samenwerk en discussies. Bij IMARES/WMR ben ik studente, gastmedewerkster, onderzoeksassistente, vrijwilligster en aio geweest en uiteindelijk mag ik me onderzoekster noemen. Voor alle kansen die IMARES/WMR me bod wil ik me graag bedanken! Roel en Pascalle, met jullie mocht ik een spannend jaar samenwerken als onderzoeksassistent bij een project over primaire productie. Ik heb van jullie geleerd om zeer kritisch naar onderzoeksdata te kijken en geen apparaat blind te vertrouwen. Dit heeft mij erg geholpen tijdens mijn tijd als aio en zal hopelijk ook in de toekomst nuttig zijn. Alma, jij was in staat om mij (herhaaldelijk) uit fases van aio-zelfmedelijden te halen. Je zet dingen in een ander perspectief, dat heb ik altijd als ontzettend nuttig en belangrijk ervaren. Ik wil je ook bedanken voor diverse wollen truien, die zonder onze gezamenlijke breiavonden nooit waren ontstaan. Er is een iemand, die je zonder moeite als het sociale smeermiddel van de afdeling Ecosystemen op Texel kunt omschrijven: Willem! Lieve Willem, jouw vertrek bij IMARES was waarschijnlijk de juiste keuze en toch een echte verlies voor ons allen! Niemand die ik ken is zo attent. Jouw vele lieve kaarten vullen muren in mijn huis, jij hebt altijd de passende woorden paraat! Naar jouw bezoeken op de ZVS kijk ik altijd uit, dank voor alle titel(blad)inspiratie in de afgelopen maanden! Marlies en Iris, jullie weten altijd raad en zijn heerlijk gezellig, dank voor jullie hulp en ondersteuning vanuit het secretariaat. Mardik, oh Mardik, het enthousiasme over (zeevogel)onderzoek spat van je af, op die manier besmette je me ook met het interesse in zeevogeldieet met al zijn fascinerende otolieten! Je relaxte blik op het leven vind ik erg bijzonder, er valt van alles van je te leren! Door onze voetwebstudies zijn de dagelijkse veertochten in de zomer nooit verspilde tijd. Aan boord worden ook altijd interessante, leuke en leerzame gesprekken gevoerd, dank voor de gezelligheid: Hans, Martin, Matthijs, Jaap, Michaela, Geert, Jessica, Joop en Sophie.

Kees, ik heb zo veel van je mogen leren! Met jou mocht ik de meeuwenkolonie in de duinen te bezoeken, wat is dat een bijzondere plek! Ook met jou ben ik aan de slag gegaan met otolieten en meer nog met visbotjes. Uit die samenwerking is een heel mooi boek ontstaan, waar ik erg trots op ben! Dode (olie)vogels zoeken op het strand (en samen open snijden) hoort bij mijn liefste hobby's, ik hoop dat jouw databestand de komende jaren blijft groeien, een schat van ontelbare waarde voor de zeevogelbescherming in Nederland!

Andy, with the PLASTOX project you have created the opportunity for me, to work intensively on the research topic I love. The people working for PLASTOX (too many to name) were all very welcoming and helpful and I've learned a lot from you all and I enjoyed our meetings all over Europe. Andy and Lisbet, especially during the last months you two were amazingly helpful and patient with the completion of the stomach oil chapter. The deadline 
was coming very close and you've spent even weekends to go through my manuscript and provide useful comments, even on short notice. I am enjoying our cooperation so much, as I'm learning new thing every time we speak. I hope that this cooperation can continue in the future, your critical thoughts and your chemist-perspective are such an enrichment!

Een tijdje terug stond Albert ineens voor de deur. Hij wilde graag betrokken worden bij ons plasticonderzoek in stormvogels. Albert is niet zomaar iemand, we kunnen gerust stellen dat hij zo ongeveer alles over plastic weet! Albert, dankzij jouw hulp konden wij in de afgelopen jaren steeds meer richting chemische analyse van plastic werken. Jij bent altijd ontzettend gemotiveerd om nieuwe projecten aan te gaan en door je uithoudingsvermogen hebben wij ook diverse uitdagingen aangekund! Jouw bijdrage aan het PLASTOX project heeft niet alleen mij veel geholpen, alle partners hebben van je kennis en kunde kunnen profiteren! Ik heb bij Carat kunnen leren hoe je plastic met een FTIR analyseert en mee mogen kijken hoe jullie verschillende elementen in het plastic bepalen, heel leerzaam! Hier hebben ook jouw collega's, Stephie, André en Erwin essentieel aan bij gedragen, door vele uren in het lab nieuwe methodes te ontwikkelen, te testen en uit te voeren! Ook jullie samenwerking met SHIMADZU was ontzettend belangrijk voor ons. Marion, du hast so viel Geduld mit uns 'Biologen' bewiesen! Vielen Dank, dass du uns bei SHIMADZU regelmäßig eure Türen geöffnet hast, um uns bei verschiedenen Analyseprojekten zu helfen! Zonder jullie waren het PLASTOX project en mijn proefschrift nooit zo ver gekomen!

Dear thesis reading commission, Tinka, Paula, Juliana and Eric, I am very grateful that you have agreed to read my thesis. Thank you very much for your time and effort, I'm looking forward to discussing the thesis with you!

Dick, thuis kwam ik geregeld erg mooie proefschriften tegen, die door jou opgemaakt waren. Ik droomde ervan om ook zo een mooi boek te mogen hebben, en gelukkig heb je toegezegd om mij te helpen. De lay-out van de hoofdstukken ziet er ontzettend gaaf uit, dankjewel!

Nils und Stefan vom FTZ Büsum, bei euch ist eigentlich alles begonnen! Mit und dank sei euch war ich die ersten Male mit einem Forschungsschiff auf See. Da, wo die echten Vögel wohnen! Zusammen mit euch habe ich meine ersten Eissturmvögel bewundert, diese neugierigen Vögel, mit den langen, brettsteifen Flügeln und den leuchtenden Augen. Eure Begeisterung und die Liebe zu Seevögeln waren eindeutig ansteckend, vielen Dank dafür. Zum Glück konnten wir auch nach meinem Praktikum zusammenarbeiten, hoffentlich bleibt diese Zusammenarbeit noch lange bestehen!

Before my $\mathrm{PhD}$ project started in 2016, I was lucky enough to spend a year in the beautiful town of Ísafjörður (Iceland) and to meet people that belong to the best and most important friends I could wish for! I'm very grateful for the wonderful time we spent together in Ísafjörður but I want to thank a few of you awesome people in particular, as you helped me a lot 
throughout my PhD. Alan, you and your perfect English were extremely helpful when I needed help with English grammar. On numerous occasions you are my grammar hero, replying quickly to all of my requests, even if these were quite big ones as the correction of my thesis Introduction. Another spontaneous English rescuer was Ellyn, you took the time to check my thesis Discussion, a lot of work, as you did not only check for grammar but also helped me with sorting my thoughts! Great help came from little Chris, Chris I love your amazing, genius and funny mind! Your capability to explain math to me, made me really enjoy math classes, something very uncommon in my previous life! Anja, our several Skype discussions often helped in finding new ideas and viewpoints, I'm very fortunate to have you as an honest and caring friend! Thank you and Halldór for having me at your home in snowy and stormy Ísafjörður, after the submission of this thesis, exact the kind of holiday I needed! Halldór, takk fyrir for teaching me everything about kayaking. Who would have thought that kayaking is so important to understand life in general!

Als aio blijft er weinig tijd over voor dingen, die niet met je proefschrift te maken hebben. Die schaarse vrije tijd heb ik grotendeels onder een kajak in het water hangend doorgebracht. Gelukkig ben ik niet de enige met zo'n gek hobby. Op Texel zijn door het eskimoteren hele kostbare vriendschappen ontstaan met vooral Rob, Flip \& Cynthia. Ik dank jullie ontzettend voor alle afleiding, enthousiasme, lekker eten en gezelligheid! Ook buiten Texel bestaat er een groep van rollers waarbij ik me enorm thuis voel. De groep blijft groeien maar ik wil toch graag Rein \& Manon, Julit, Marijn, André, David, Just, Gerard, Max \& Annelies danken voor alle goede tips en gezellige (winterse) oefendagen ergens in het land!

Op Texel kon ik altijd terecht bij Eva \& Tjibbe (en kleine Ben). Jullie zorgden regelmatig voor goed eten (en de afwas!), leuke uitstapjes, goede gesprekken en gezelligheid. Jullie kennis over de beste plekken voor appeltaart op het eiland was altijd handig! Bovendien mag ik jullie binnenkort verwelkomen als nieuwe Oosterenders, ik kan niet wachten!

Stephan und Mutti, euch war Bildung immer wichtig! Ihr habt drei wunderbare Kinder erzogen und uns alle Möglichkeiten geboten. Eure Liebe und Fürsorge hat mir den Mut gegeben, neue Welten zu erkunden. Egal wie verrückt meine Pläne waren, ihr habt sie unterstützt und gefördert! Wo auch immer auf der Welt diese Pläne ausgeführt wurden, ihr habt sie (und mich) besucht und habt euch mit begeistern lassen! Um es mit den Worten Herman van Veens zu sagen: Jullie zijn het veiligste, wat mij in het verwarrend mooie leven is overkomen.

Job, het toeval wil, dat de leukste baan die er is en de leukste man die er is op een klein eiland voor de Nederlandse kust bij elkaar kwamen. Al meer dan 10 jaar hou ik ontzettend veel van jou! We hebben samen (met het katertje) een erg mooi leven opgebouwd, waarvan ik iedere dag opnieuw geniet! Voor jouw vertrouwen in mij, ook al moest ik soms even een jaar naar IJsland, ben ik je erg dankbaar! Jij hebt me door de wat donkere tijden gedragen en ook in de aio jaren was jij mijn grote steun! Dank voor alles, jouw hulp heeft enorm ertoe bijgedragen het proefschrift op tijd af te krijgen. 


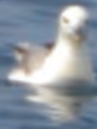

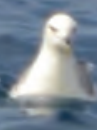

\section{8}

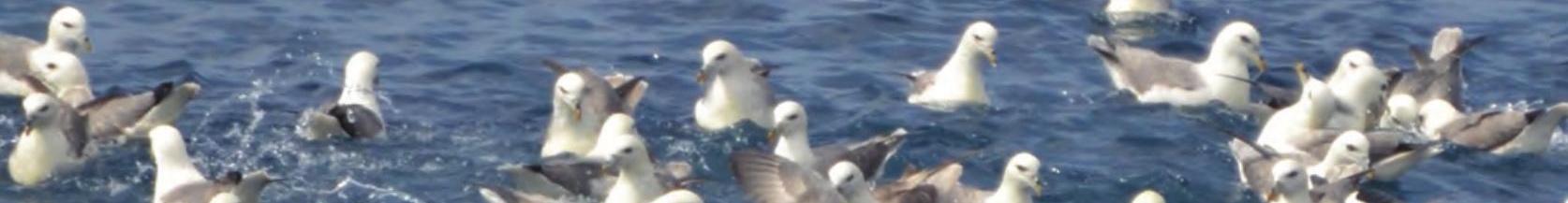

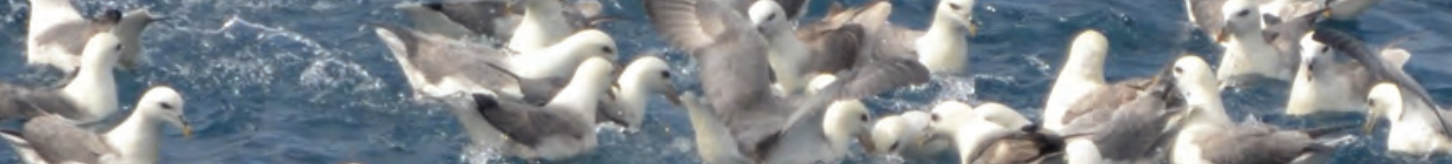

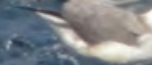

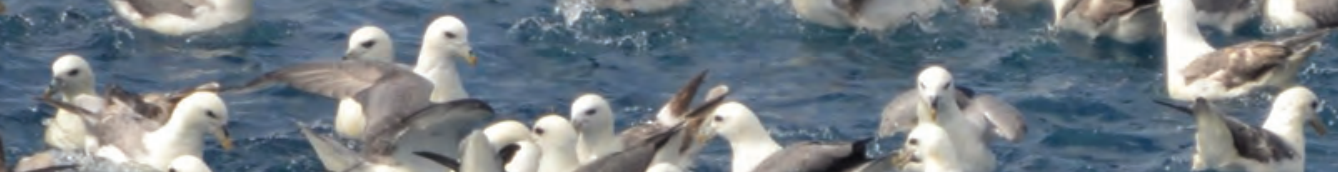

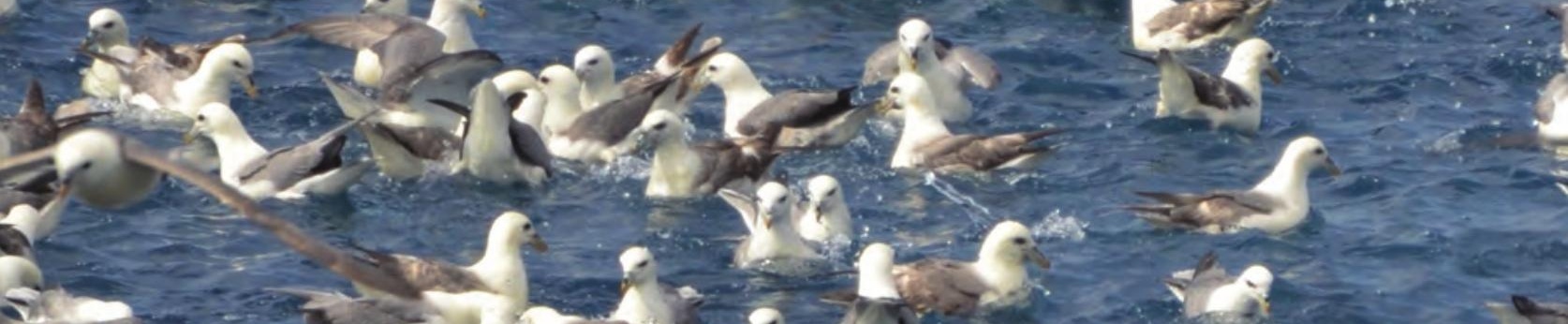

$2 e^{2}$

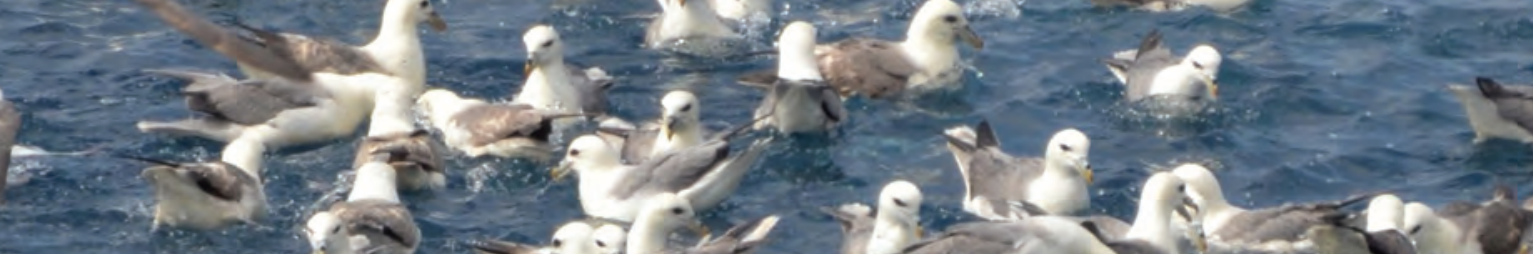

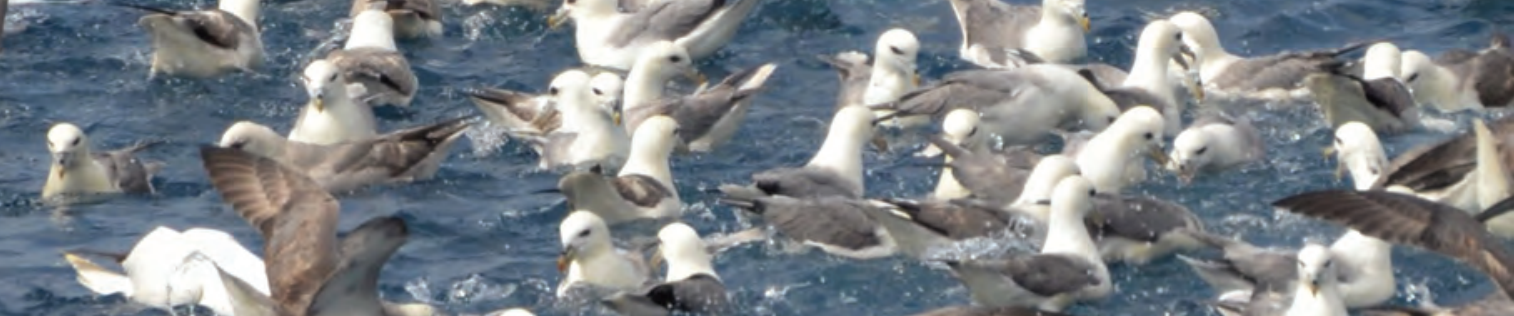

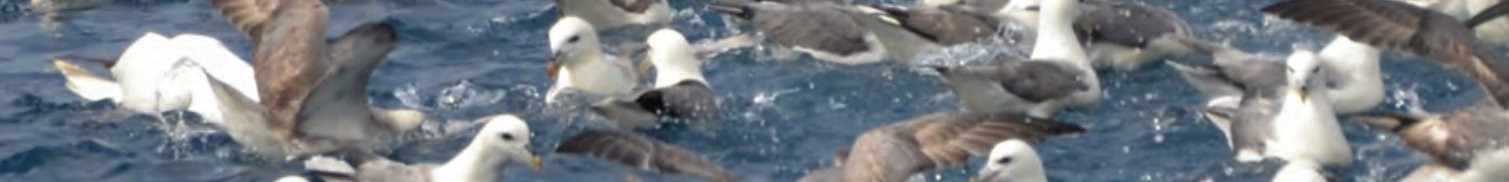

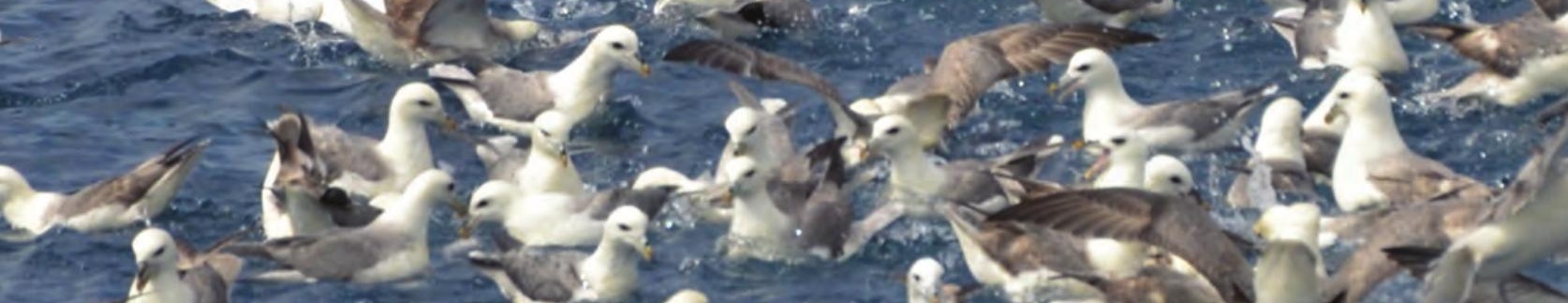

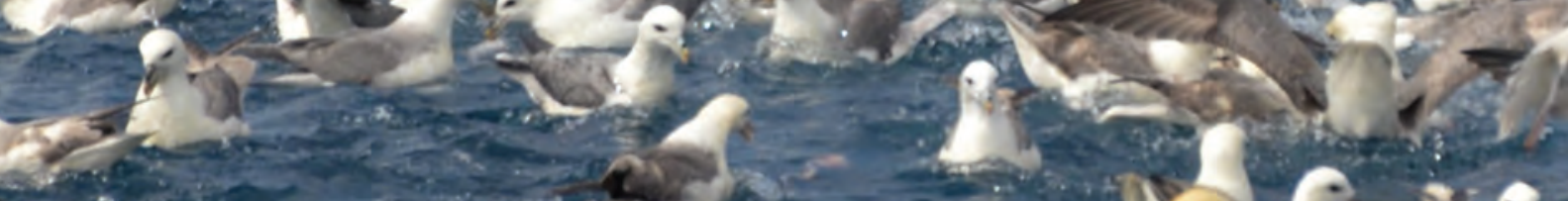
e.
$x+9$

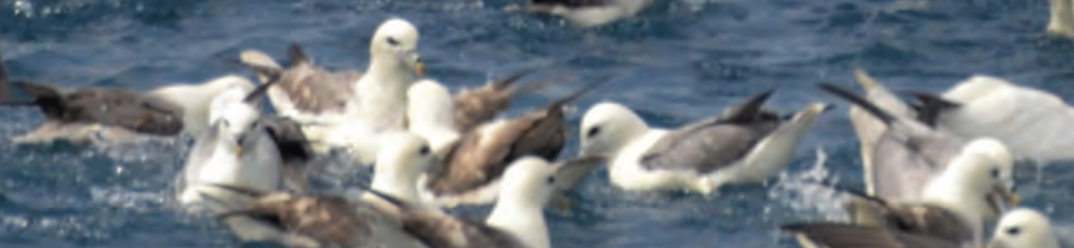




\section{4}

\section{Author affiliations}

$$
\text { s. } s
$$

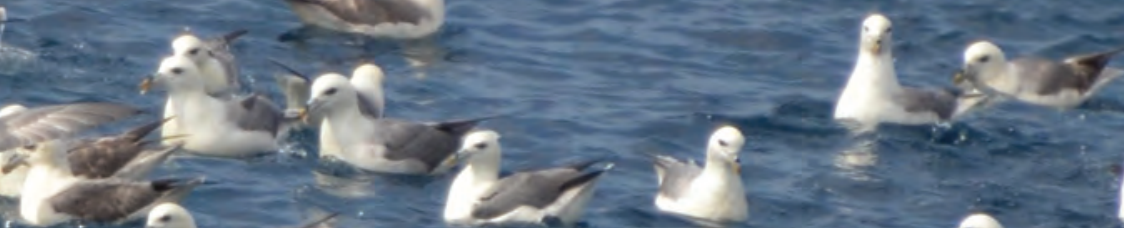

$3^{2} \mathrm{~d} 2 \mathrm{C}$

\section{2}

2. 598<smiles>C1=C[C@H]2C[C@H]1C2</smiles>

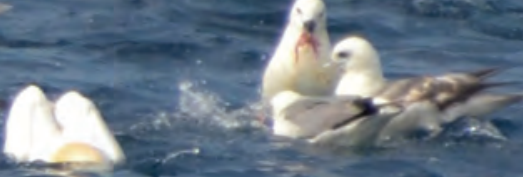

$x^{3}-3$

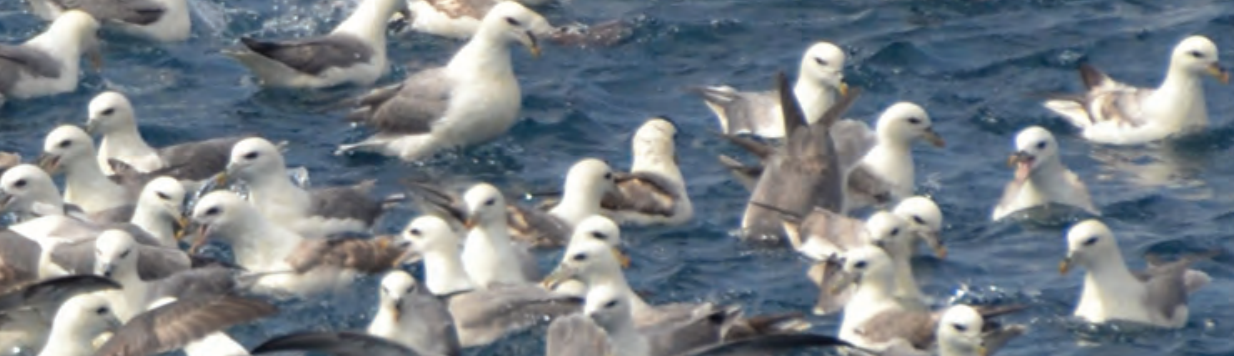

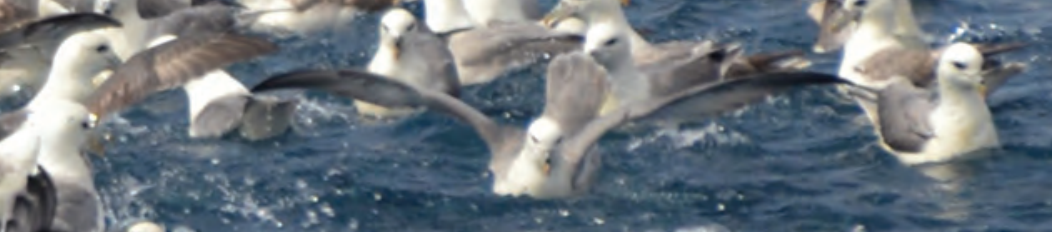


Amalie V. Ask

Norwegian Polar Institute

Hjalmar Johansens gate 14

9296 Troms $\varnothing$

Norway

amalie.ask@npolar.no

\section{Melanie Bergmann}

Alfred-Wegener-Institut, Helmholtz-Zentrum

für Polar- und Meeresforschung

Am Handelshafen 12

27570 Bremerhaven

Germany

melanie.bergmann@awi.de

\section{Andy M. Booth}

SINTEF Ocean

Brattørkaia 17C

7465 Trondheim

Norway

andy.booth@sintef.no

\section{Elisa L. Bravo Rebolledo}

Varkensmarkt 9,

4101 CK Culemborg

The Netherlands

e.l.bravorebolledo@buwa.nl

\section{Marion Egelkraut-Holtus}

Shimadzu Europa GmbH

Albert-Hahn-Str. 6-10

47269 Duisburg

Germany

meh@shimadzu.eu

\section{Hauke Flores}

Alfred-Wegener-Institut, Helmholtz-Zentrum für Polar- und Meeresforschung

Am Handelshafen 12

27570 Bremerhaven

Germany

hauke.flores@awi.de

\section{Edwin Foekema}

Wageningen Marine Research

Ankerpark 27

1781 AG Den Helder

The Netherlands

edwin.foekema@wur.nl

\section{Geir Wing Gabrielsen}

Norwegian Polar Institute

Hjalmar Johansens gate 14

9296 Tromsø

Norway

geir.wing.gabrielsen@npolar.no

\section{Enya Hermsen}

Wageningen University \& Research P.O. Box 47

6700 AA, Wageningen

The Netherlands

enyahermsen@gmail.com

\section{Susanne Kühn}

Wageningen Marine Research

Ankerpark 27

1781 AG Den Helder

The Netherlands

susanne.kuehn@wur.nl

\section{Han Lindeboom}

Wageningen University \& Research

P.O. Box 47

6700 AA, Wageningen

The Netherlands

han.lindeboom@wur.nl

\section{André Meijboom}

Wageningen Marine Research

Ankerpark 27

1781 AG Den Helder

The Netherlands

andre.meijboom@wur.nl

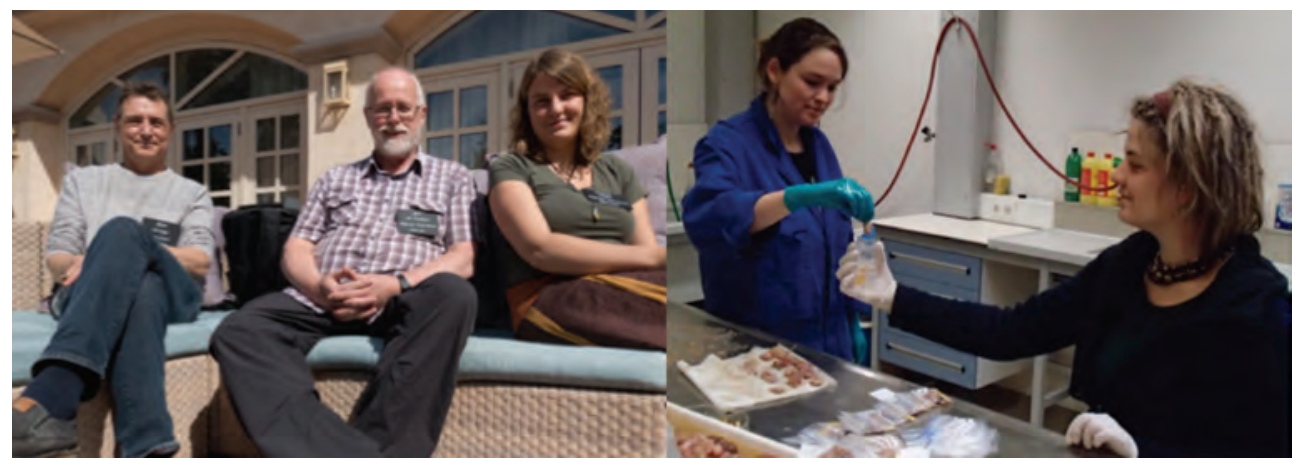


Anastasia O' Donoghue

University of Utrecht

Princetonlaan 8a,

3584 CB Utrecht

The Netherlands

anastasia.odonoghue@googlemail.com

\section{Fokje Schaafsma}

Wageningen Marine Research

Ankerpark 27

1781 AG Den Helder

The Netherlands

fokje.schaafsma@wur.nl

\section{Lisbet Sørensen}

SINTEF Ocean

Brattørkaia 17C

7465 Trondheim

Norway

lisbet.sorensen@sintef.no

\section{Marrit Starkenburg}

University of Applied Science Van Hall

Larenstein P.O. Box 1528

8901 BV, Leeuwarden

The Netherlands

marritstarkenburg@gmail.com

\section{Ailynn Swiers}

University of Applied Science Van Hall

Larenstein P.O. Box 1528

8901 BV, Leeuwarden

The Netherlands

ailynn94@hotmail.com
Mine B. Tekman

Alfred-Wegener-Institut, Helmholtz-Zentrum

für Polar- und Meeresforschung

Am Handelshafen 12

27570 Bremerhaven

Germany

mine.banu.tekman@awi.de

\section{Alice Trevail}

University of Liverpool,

Brownlow Hill,

L69 7ZX Liverpool

United Kingdom

alice.trevail@gmail.com

Jan Andries van Franeker

Wageningen Marine Research

Ankerpark 27

1781 AG Den Helder

The Netherlands

jan.vanfraneker@wur.nl

Albert van Oyen

CARAT GmbH

Harderhook 22

46395 Bocholt

Germany

albert.van.oyen@carat-lab.com

\section{Bernike van Werven}

University of Utrecht

Heidelberglaan 2

3584 CS Utrecht

The Netherlands

bernikevanwerven@outlook.com

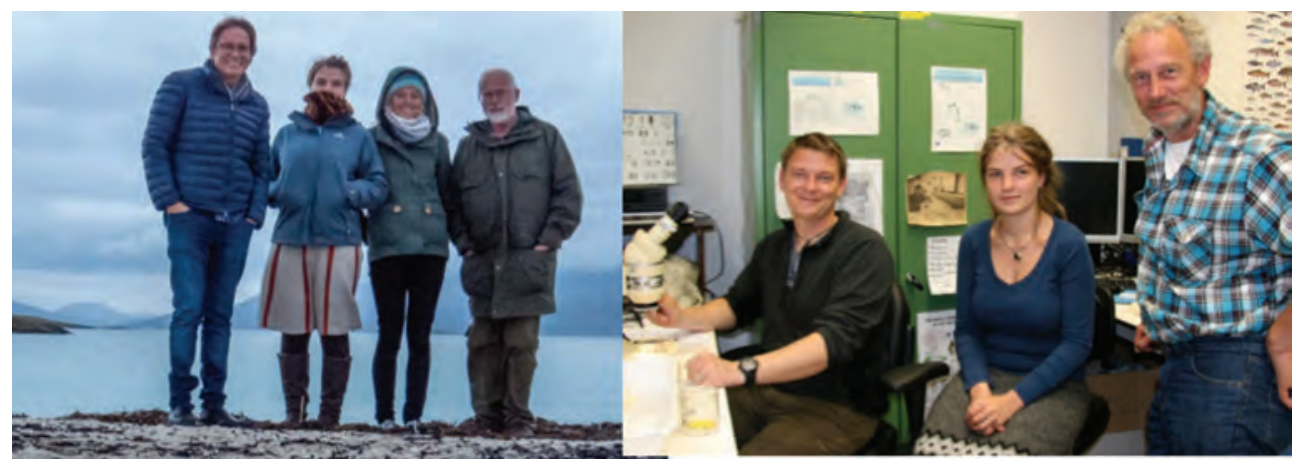





\section{List of publications}




\section{Scientific books, articles and chapters}

\section{0}

Kühn S \& van Franeker JA (2020) Quantitative overview of marine debris ingested by marine megafauna. Marine Pollution Bulletin 151: 110858

Kühn S, van Franeker JA, O'Donoghue AM, Swiers A, Starkenburg M, van Werven B, Foekema E, Hermsen E, Egelkraut-Holtus M, Lindeboom H (2020) Details of plastic ingestion and fibre contamination in North Sea fishes. Environmental Pollution 257: 113569

\section{9}

Provencher JF, Borelle S, Bond AL, Lavers JL, Van Franeker JA, Kühn S, Hammer S, Avery-Gomm S, Mallory ML (2019) Recommended best practices for plastic and litter ingestion studies in marine birds: collection, processing, and reporting. Facets 4: 111-130

\section{8}

Kühn S, van Oyen A, Booth AM, Meijboom A, van Franeker JA (2018) Marine Microplastic: Preparation of Relevant Test Materials for Laboratory Assessment of Ecosystem Impacts. Chemosphere 213: 103-113

Kühn S \& Leopold MF (2018) De 'Voetprint' van Een Gespecialiseerd Gewoontedier: Kleine Mantelmeeuw Eet Grote Zeenaalden. Sula: 1-4

Kühn S, Schaafsma FL, van Werven B, Flores H, Bergmann M, Egelkraut-Holtus M, Tekman MB, van Franeker JA (2018) Plastic Ingestion by Juvenile Polar Cod (Boreogadus saida) in the Arctic Ocean. Polar Biology

\section{7}

Van Franeker JA, Rebolledo ELB, Hesse E, IJsseldijk LL, Kühn S, Leopold M, Mielke L (2017) Plastic Ingestion by Harbour Porpoises Phocoena phocoena in the Netherlands: Establishing a Standardised Method. Ambio: 1-11

Kühn S, van Werven B, van Oyen A, Meijboom A, Bravo Rebolledo EL, van Franeker JA (2017) The Use of Potassium Hydroxide (KOH) Solution as a Suitable Approach to Isolate Plastics Ingested by Marine Organisms. Marine Pollution Bulletin 115: 86-90

Provencher JF, Bond AL, Avery-Gomm S, Borrelle SB, Bravo Rebolledo EL, Hammer S, Kühn S, Lavers JL, Mallory ML, Trevail A, van Franeker JA (2017) Quantifying Ingested Debris in Marine Megafauna: A Review and Recommendations for Standardization. Analytical Methods 9: 1454-1469

Camphuysen CJC \& Henderson P (with major contributions from E. Velilla, S. Kühn, M. Leopold and R. Somes) (2017) North Sea Fish and their Remains. NIOZ and Pisces Conservation Ltd, pp 326.

\section{5}

Kühn S, Bravo Rebolledo EL, van Franeker JA (2015) Deleterious effects of litter on marine life. In: Bergmann M, Gutow L, Klages M (eds) Marine Anthropogenic Litter. Springer International Publishing, Cham, pp 75-116

Besseling E, Foekema EM, Van Franeker JA, Leopold MF, Kühn S, Bravo Rebolledo EL, Heße E, Mielke L, IJzer J, Kamminga P (2015) Microplastic in a Macro Filter Feeder: Humpback Whale Megaptera novaeangliae. Marine Pollution Bulletin 95: 248-252

Trevail AM, Gabrielsen GW, Kühn S, Van Franeker JA (2015). Elevated levels of ingested plastic in a high Arctic seabird, the northern fulmar (Fulmarus glacialis). Polar Biology 38: 975-981

\section{2}

Kühn S \& Van Franeker JA (2012). Plastic ingestion by the Northern Fulmar (Fulmarus glacialis) in Iceland. Marine Pollution Bulletin 64: 1252-1254 


\section{Reports}

Baptist MJ, Volwater J, van Hal R, van Zwol J, Troost K, Van Franeker JA, Kühn S, Strietman WJ (2020) Meetrapport verzamelen van plastics van MSC Zoe: zeevogels, vissen, zeebodem, stranden - Beknopt verslag van werkzaamheden in 2019. Wageningen Marine Research, Den Helder, The Netherlands, pp 26

Van Franeker JA \& Kühn S (2019) Fulmar Litter EcoQO monitoring in the Netherlands - Update 2018. Wageningen Marine Research Report C077/19 \& RWS Centrale Informatievoorzieningen BM 19.16, pp 60

Van Franeker JA, Kühn S, Kotterman M, Kwadijk C (2019) Monitoring Van Paraffine-Achtige Stoffen Op Nederlandse Stranden En in Magen Van Noordse Stormvogels. Wageningen Marine Research Rapport C001/19, Den Helder, the Netherlands. pp 32

Leopold MF, Kik M, van Tulden P, van Franeker JA, Kühn S, Rijks J (2019) De Zoe en de zeekoet. Een onderzoek naar de doodsoorzaak en de herkomst van de zeekoeten die massaal strandden op de Nederlandse kust in januari en februari 2019. Wageningen Marine Research, Den Helder, The Netherlands, pp 59

Baptist MJ, Brasseur SMJM, Foekema EM, Van Franeker JA, Kühn S, Leopold MF (2019) Mogelijke ecologische gevolgen containerramp MSC Zoe voor Waddenzee en Noordzee - een quickscan. Wageningen Marine Research, Den Helder, The Netherlands, pp 19

Van Franeker JA \& Kühn S (2018) Fulmar Litter EcoQO monitoring in the Netherlands - Update 2017. Wageningen Marine Research Report C060/18 \& RWS Centrale Informatievoorziening BM 18.20, Den Helder, pp 60

Leopold MF, Geelhoed SCV, Verdaat H, Kühn S, van Puijenbroek M (2018) Reacties zeevogels in windparken bij doorvaart. Wageningen Marine Research, Den Helder, The Netherlands, pp 32

Van Franeker JA, Kühn S, Pedersen J, Hansen PL (2017) Fulmar Litter EcoQO monitoring in Denmark 2002-2016. Report for the Danish Environmental Protection Agency. Wageningen Marine Research Den Helder, The Netherlands, pp 24

Van Franeker JA, Kühn S, Bravo Rebolledo EL (2016) Fulmar Litter EcoQO monitoring in the Netherlands - Update 2015. Wageningen Marine Research Den Helder, The Netherlands, pp 50

Brinkman AG, Riegman R, Jacobs P, Kühn S, Meijboom A (2015) Ems-Dollard Primary Production Research Full Data Report. IMARES, Texel, The Netherlands

Trevail AM, Kühn S, Gabrielsen GW (2015) The State of Marine Microplastic Pollution in the Arctic. Kortrapportserie / Brief Report Series no. 033. Norwegian Polar Institute, Tromso, Norway, pp 24

Van Franeker JA, Kühn S, Bravo Rebolledo EL, Meijboom A (2014) Fulmar Litter EcoQO monitoring in the Netherlands - Update 2012 and 2013. IMARES Report C122/14, Texel, The Netherlands, pp 56

Trevail AM, Gabrielsen GW, Kühn S, Bock A, Van Franeker JA (2014). Plastic Ingestion by Northern Fulmars, Fulmarus glacialis, in Svalbard and Iceland, and Relationships between Plastic Ingestion and Contaminant Uptake. Kortrapportserie / Brief Report Series no. 029. Norwegian Polar Institute, Tromsö, pp 24

Leopold MF, Van Bemmelen R, Kühn S, Lagerveld S, (2012). Friese Front Alk/Zeekoet: Oktober/ November 2012, Cruise Rapport. IMARES Rapportnummer C150/12, IMARES, Texel, pp 27 


\section{SENSE}

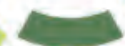

Netherlands Research School for the

Socio-Economic and Natural Sciences of the Environment

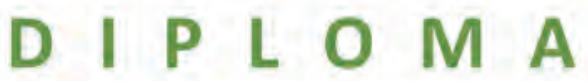

for specialised PhD training

The Netherlands research school for the

Socio-Economic and Natural Sciences of the Environment

(SENSE) declares that

\section{Susanne Kühn}

born on 22 January 1987 in Schkeuditz, Germany

has successfully fulfilled all requirements of the educational PhD programme of SENSE.

Wageningen, 30 October 2020

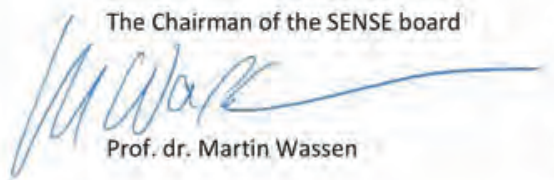

the SENSE Director of Education

The SENSE Research School has been accredited by the Royal Netherlands Academy of Arts and Sciences (KNAW)

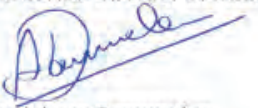

Dr. Ad van Dommelen

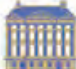

K O N I N K L I J K E N E I E R L A N D S E

A K A D E M I E V A N W E T E N S C H A P P E N 


\section{SENSE}

The SENSE Research School declares that Susanne Kühn has successfully fulfilled all requirements of the educational PhD programme of SENSE with a work load of $66.0 \mathrm{EC}$, including the following activities:

\section{SENSE PhD Courses}

Environmental research in context (2016)

- Research in context activity: Initiating and co-organizing lectures and group activities to strengthen team and institute community exchange (2016-2018)'

\section{Selection of Other PhD/MSc and general skill Courses}

Plastic analysis techniques (FTIR-ATR, microFTIR, EDX), Carat/Shimadzu, Germany (2016)

Basic Statistics, PE\&RC and SENSE (2018)

Introduction to R, PE\&RC and SENSE (2018)

Instruction course for weapon handling, safety regulations, and protection of and against polar bears, AWI, Germany (2017)

Top Predator survey Training in the Arctic, WMR/AWI, North Pole (2017)

Safety at sea, Falck, The Netherlands (2018)

Emergency Breathing systems (EBS), DHTC, The Netherlands (2018)

Top Predator survey Training in the Antarctic, WMR/AWI, South Pole, (2018-2019)

\section{Selection of Outreach and societal impact activities}

MSFD Threshold Meeting: Updating policy guidance, TCGML, Berlin

TV Interview: NZZ Format "Der Müll und das Meer", Swiss/German Television (2017)

Interview Radio „Quarks”: Ballons und Umweltverschmutzung, WDR 5, Germany (2019)

\section{Selection of Management and Didactic Skilis Training}

Co-organiser Fulmar dissection workshop 2018

Secretary Dutch Seabird Group and Editor of its journal SULA (2018-2020)

Member of the Specialist Committee on Seabirds and Plastic Pollution under the World Seabird Union (2017-2020)

D. Supervising several BSc students (2017-2018) and MSe students (2016-2019)

\section{Selection of Oral Presentations}

- Invited speaker: Northern fulmar as an indicator for marine pollution. Arctic Circle Conference, 7-9 October 2016, Reykjavik, Iceland

c. Using marine organisms to monitor plastic litter at sea, North Sea meets Baltic Sea, 20 May 2019 Warnemünde, Germany

\section{SENSE coordinator PhD education}

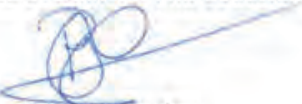

Dr. ir. Peter Vermeulen 


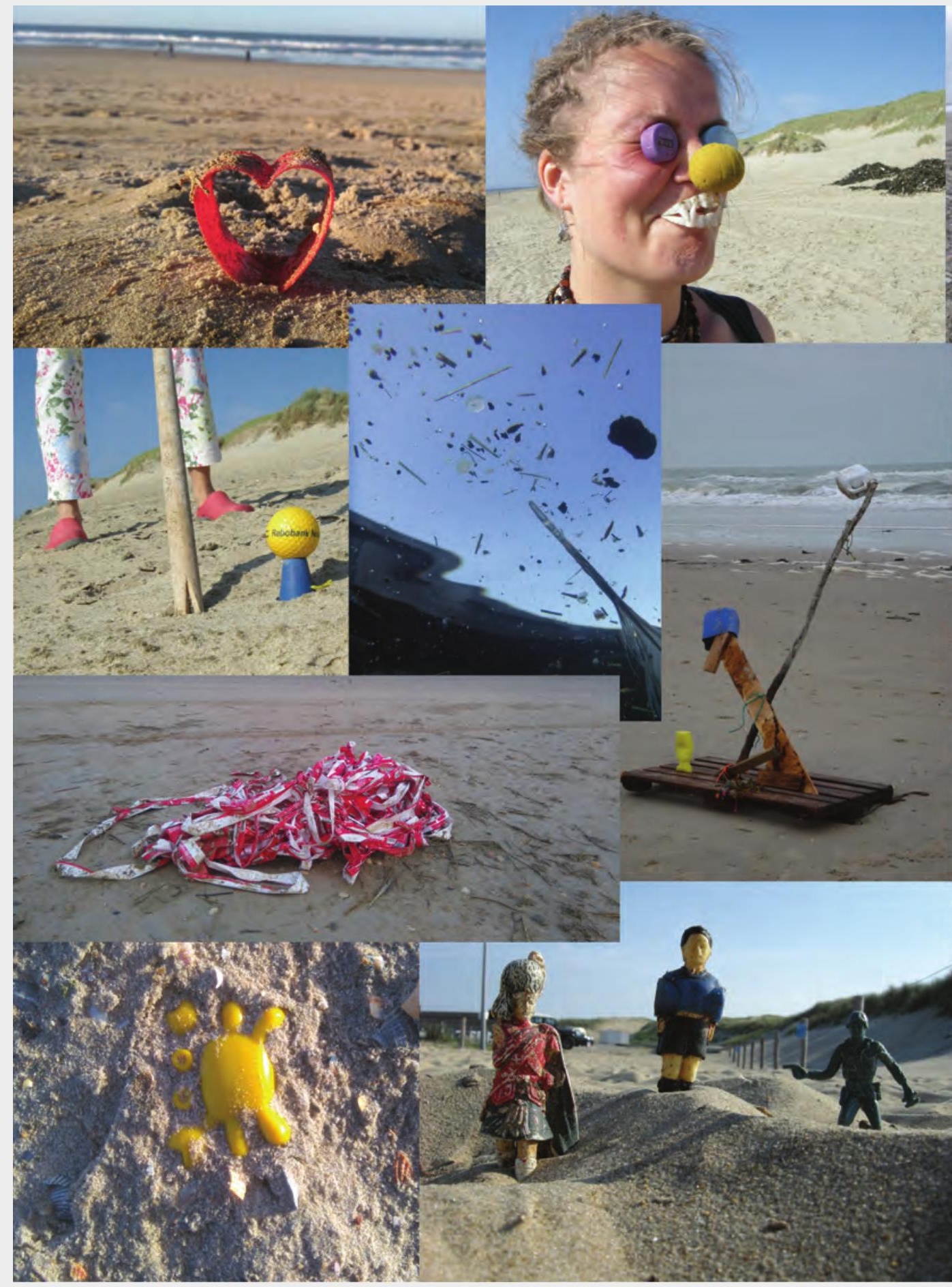




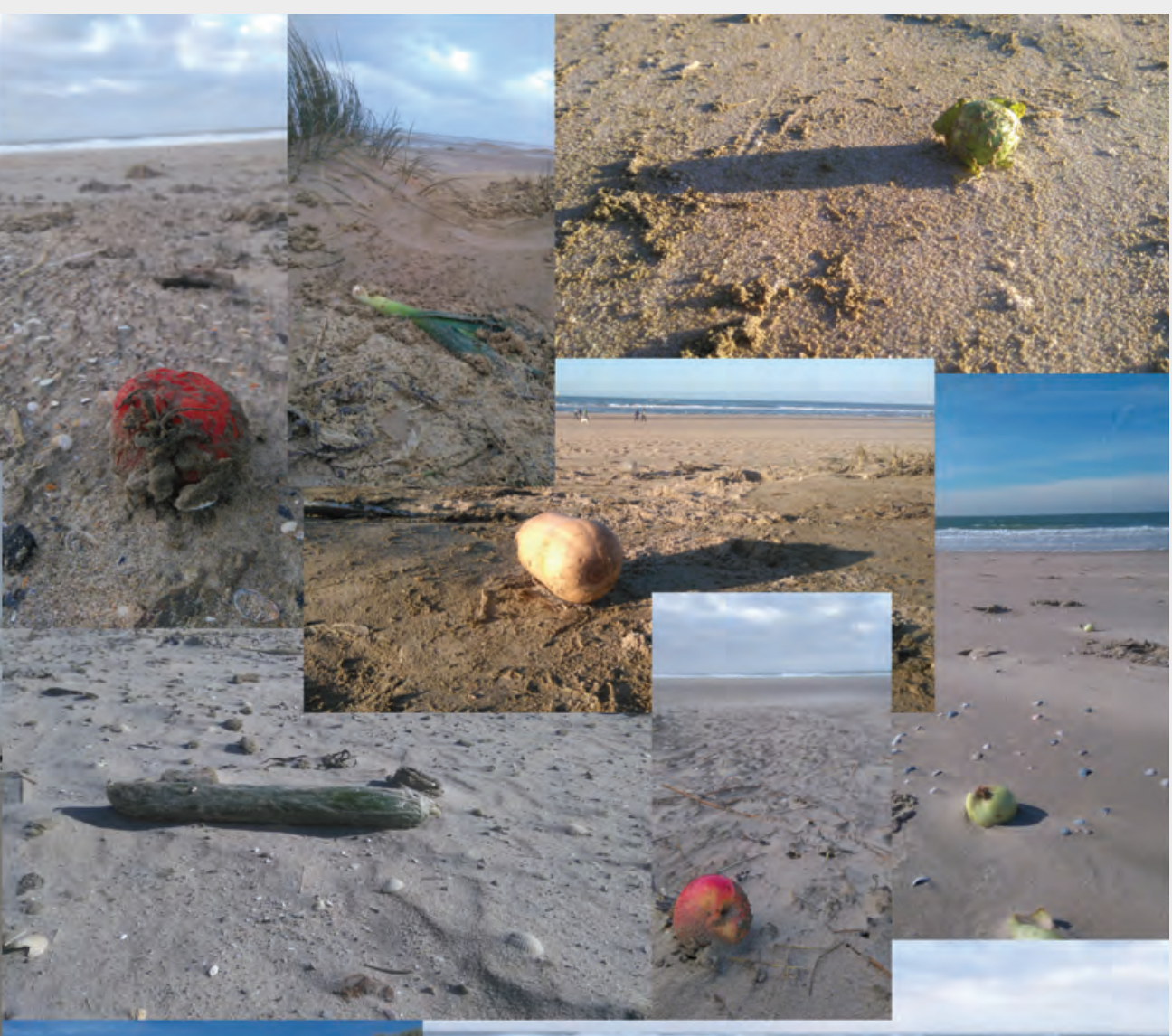

(2)

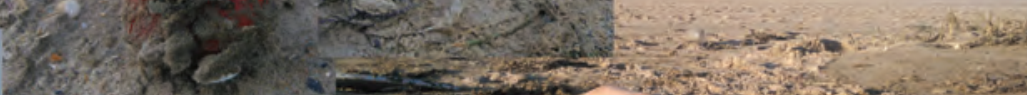

4

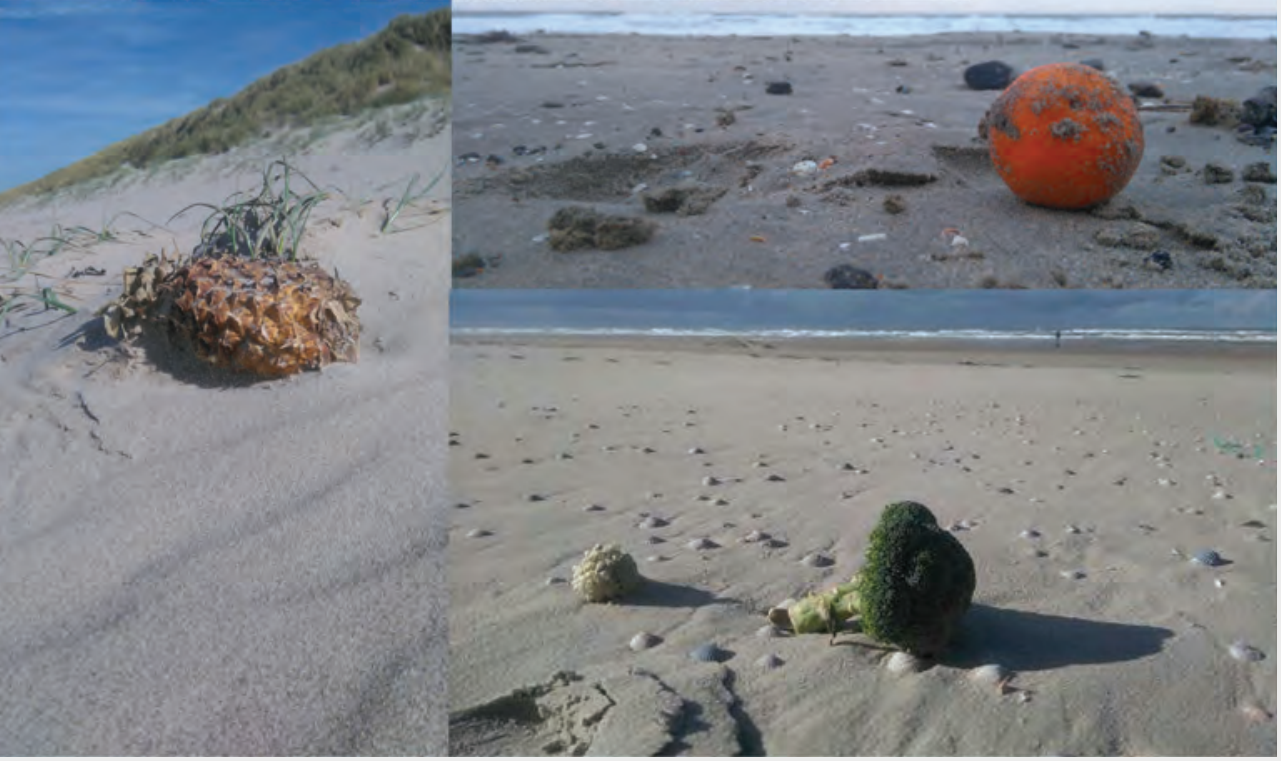


The research conducted in this thesis was financially supported by Dutch Science Foundation (project ALW-NWO 856.15.001) under EU Joint Program Initiative (JPI) 'Oceans' within the PLASTOX project (Direct and indirect ecotoxicological impacts of microplastics on marine organisms).

Financial support from Wageningen University for printing this thesis is gratefully acknowledged.

\section{COLOFON}

Lay-out and figures: Dick Visser

Photo credits: Marlous Heemstra (Page 20/21)

Hauke Flores (Page 40/41)

Jan A. van Franeker (Page 66)

SINTEF (Andy Booth) (Page 104/105)

Jan A. van Franeker, Fokje Schaafsma, Dan Turner, Anja Nickel (Page 215/215)

Printed by: DigiForce/www.proefschriftmaken.nl

Susanne Kühn

Message in a belly - Plastic pathways in Fulmars

232 pages

$\mathrm{PhD}$ thesis, Wageningen University, Wageningen, The Netherlands (2020)

With references, with summary in English, German and Dutch

ISBN: 978-94-6395-250-7

DOI: https://doi.org/10.18174/509638 



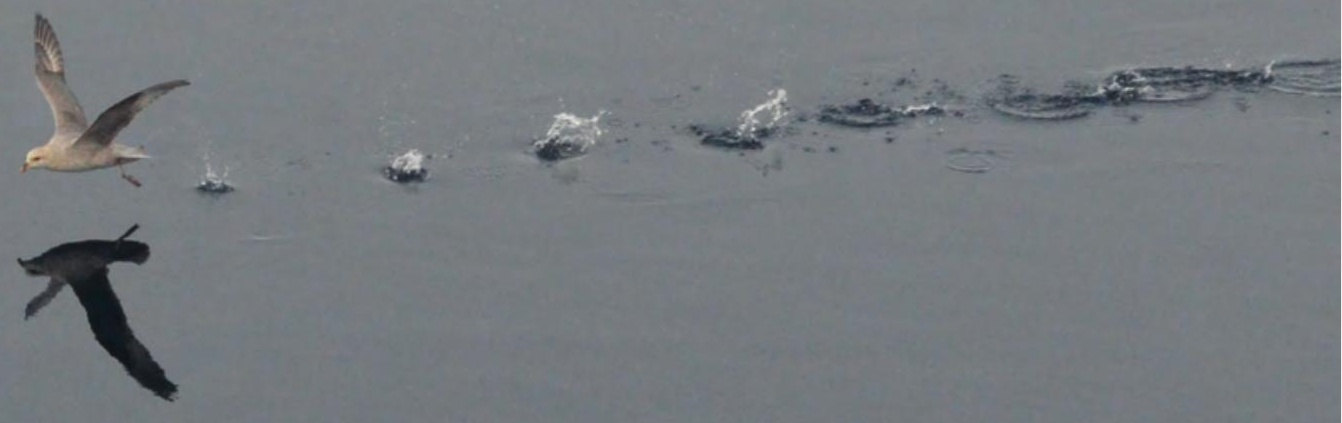

